\title{
EMG-based assessment of active muscle stiffness and co-contraction in muscles with primary and secondary actions at the wrist during piano playing
}

Caroline Andison

\begin{abstract}
A thesis submitted to the
Faculty of Graduate and Postdoctoral Affairs

in partial fulfillment of the requirements for the degree of
\end{abstract}

Master of Applied Science

Ottawa-Carleton Institute for Biomedical Engineering

Department of Mechanical and Aerospace Engineering

Carleton University

Ottawa, Ontario

May 2011

(C) Copyright

2011, Caroline Andison 


$\begin{array}{ll}\begin{array}{l}\text { Library and Archives } \\ \text { Canada }\end{array} & \begin{array}{l}\text { Bibliothèque et } \\ \text { Archives Canada }\end{array} \\ \begin{array}{l}\text { Published Heritage } \\ \text { Branch }\end{array} & \begin{array}{l}\text { Direction du } \\ \text { Patrimoine de l'édition }\end{array} \\ 395 \text { Wellington Street } & \begin{array}{l}395, \text { rue Wellington } \\ \text { Ottawa ON K1A ON4 } \\ \text { Canada }\end{array} \\ \begin{array}{l}\text { Ottawa ON K1A ON4 } \\ \text { Canada }\end{array}\end{array}$

Your file Votre référence

ISBN: 978-0-494-81672-1

Our file Notre référence

ISBN: 978-0-494-81672-1

NOTICE:

The author has granted a nonexclusive license allowing Library and Archives Canada to reproduce, publish, archive, preserve, conserve, communicate to the public by telecommunication or on the Internet, loan, distribute and sell theses worldwide, for commercial or noncommercial purposes, in microform, paper, electronic and/or any other formats.

The author retains copyright ownership and moral rights in this thesis. Neither the thesis nor substantial extracts from it may be printed or otherwise reproduced without the author's permission.
AVIS:

L'auteur a accordé une licence non exclusive permettant à la Bibliothèque et Archives Canada de reproduire, publier, archiver, sauvegarder, conserver, transmettre au public par télécommunication ou par l'Internet, prêter, distribuer et vendre des thèses partout dans le monde, à des fins commerciales ou autres, sur support microforme, papier, électronique et/ou autres formats.

L'auteur conserve la propriété du droit d'auteur et des droits moraux qui protège cette thèse. $\mathrm{Ni}$ la thèse ni des extraits substantiels de celle-ci ne doivent être imprimés ou autrement reproduits sans son autorisation.
In compliance with the Canadian Privacy Act some supporting forms may have been removed from this thesis.

While these forms may be included in the document page count, their removal does not represent any loss of content from the thesis.
Conformément à la loi canadienne sur la protection de la vie privée, quelques formulaires secondaires ont été enlevés de cette thèse.

Bien que ces formulaires aient inclus dans la pagination, il n'y aura aucun contenu manquant. 


\section{Abstract}

Prominent piano pedagogues present co-contraction as being potentially injurious while playing the instrument. However, maintaining proper arm posture requires co-contraction. The goal of this study was to quantify changes in active muscle stiffness and co-contraction in university-level pianists who played a scale, two triad exercises and a composition by Ann Southam. Co-contraction was calculated from EMG measurements of the FCU, ECU, FDS and EDC muscles.

In the scale task significant but steady levels of co-contraction were measured. Cocontraction in the triad exercises was not directly related to feelings of discomfort. During the performance of a piece composed by Ann Southam for this research subjects showed significant variations in co-contraction that corresponded to faster note rates and increased loudness. Additional experiments to further clarify relationships between note rate, dynamic level, posture and co-contraction are proposed.

Results from this study indicate the presence of co-contraction is fundamental to piano playing. 
To my grandfathers, who were both believers in the value of hard work. 


\section{Acknowledgments}

There are several people to whom I wish to extend my sincerest thanks:

First and foremost, I owe a huge thank you to my supervisor, Prof. Donald Russell, for his financial and academic support. Thank you for introducing me to the field of engineering. I will never forget our first meeting that lasted nearly three hours (as I recall), where we discussed my transition from health sciences to engineering. From that day forward you have always made time for me, whether it was taking care of administrative tasks that I dreaded or meeting with me once a week to discuss progress in my coursework and thesis. You created a positive learning environment for me, while constantly offering me encouragement and delivering guidance and feedback. Thank you for being "human," and being able to sense when I was feeling discouraged. You have been more than an ideal supervisor for me.

Thank you to Dr. Gilles Comeau for allowing me to use your lab and for trusting me with the use of your EMG equipment. You made me feel welcome and showed interest in what I was working on. I also wish to thank past and present members of the Piano Pedagogy Research Laboratory: Zacharie Brunet, YiFei Liu, Milada Medinić, Flora Nassrallah, Allyshia Sewdat, Kimberley Sundell, Jada Watson, Lu Yuanyuan and Xi Zhang.

Thank you to my parents, Leslie Scott and Martin Andison. You have always emphasized that education is more important than a paycheque. Thank you for 
encouraging me to go back to school and for supporting me in every way possible throughout my degree. Thank you for being patient with me throughout this process and offering encouragement when I needed it. And thank you for staying out of my way when I was stressed out, and simply needed some peace and quiet to work. You both have achieved so much in your lives. I hope that one day I can live up to the example you have set. To my brother, Jeremy Andison, thank you for your help with programming the MIDI relay. You will always be my go-to-guy for matters concerning music and technology.

Thank you to my friends, who remained my friends no matter how unpleasant I was to be around. In particular I wish to acknowledge Elizabeth Toller, Rachelle Forsyth and Kyle Mulligan. Liz, you have been a mentor, my tireless cheerleader, a voice of reason and an unending source of support. Thank you for your wise advice on matters related and unrelated to grad school, and thanks for always "checking in" to make sure everything was okay. You continue to inspire me with your wealth of accomplishments. You will always be someone I look up to (literally and figuratively). Rachelle, you were my first friend in the biomedical program, and I have missed having you in the lab for the last year and a half. Thank you for always being so willing to lend a hand. And thank you for all of your help in troubleshooting my problems with ITEX. Kyle, you introduced me MATLAB. I would not have gotten as far as I did without your help.

Last, thank you to my boyfriend, Andrew Giallonardo. Thank you for constantly reminding me to "stay dedicated." You have always been extremely patient with me, and I realize that putting up with me for the past few months wasn't the easiest thing to do. Thank you for always encouraging open communication, and taking the time to talk things through with me, whether they were personal matters or concepts related to engineering. I have learned a lot from you. 


\section{Table of Contents}

Abstract $\quad$ iii

$\begin{array}{ll}\text { Acknowledgments } & \text { v }\end{array}$

Table of Contents vii

List of Tables $\quad$ xiii

List of Figures $\quad$ xvii

List of Abbreviations $\quad$ xxvii

1 Introduction $\quad 1$

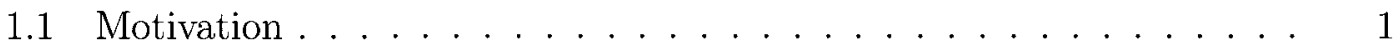

1.2 Problem Statement . . . . . . . . . . . . . . . . 2

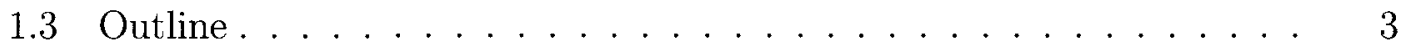

2 Background and Literature Review 5

2.1 Anatomy and Physiology Background . . . . . . . . . . . . . 6

2.1.1 Muscles - Structure and Function . . . . . . . . . . 6

2.1.2 The Forearm, Wrist, Hand and Fingers . . . . . . . . . . . 10

2.2 Electromyography . . . . . . . . . . . . . . . . . . 17

2.3 Stiffness . . . . . . . . . . . . . . . . . . . . . . 19 
2.3 .1 Muscle Stiffness . . . . . . . . . . . . . . . . . . . . . . 19

2.3 .2 Joint Stiffness . . . . . . . . . . . . . . . . . . . . . . 21

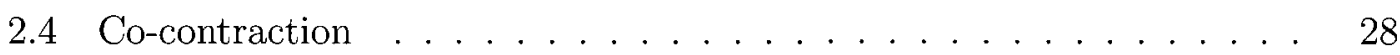

2.4.1 Quantifying Co-contraction . . . . . . . . . 30

2.4.2 Studies Using Co-contraction $\ldots \ldots \ldots \ldots$

2.5 Previous Work by Vant . . . . . . . . . . . . . . . . 38

3 Experimental Approach $\quad 41$

3.1 Participants . . . . . . . . . . . . . . . 41

3.2 Equipment. . . . . . . . . . . . . . . . . 42

3.3 Setup. . . . . . . . . . . . . . . . . 44

3.4 Task . . . . . . . . . . . . . . . . . 47

4 Data Processing and Preparation $\quad 50$

4.1 EMG processing in EMGworks ${ }^{\circledR} \ldots \ldots \ldots \ldots \ldots$

4.2 Analysis of EMG and MIDI files in the MATLAB ${ }^{\circledR}$ Environment . . 51

4.2.1 Manipulation of EMG and MIDI files in MATLAB ${ }^{\circledR} \ldots . .51$

4.2 .2 Synchronizing MIDI and EMG data . . . . . . . . . . 53

4.2 .3 Identifying sections in $\mathrm{C}$ major scale $\ldots \ldots \ldots \ldots$

4.2 .4 Identifying sections in Ann Southam piece . . . . . . . 55

5 Task \#1: Results and Discussion $\quad 59$

5.1 Results of Task \#1: C Major Scale . . . . . . . . . . . . 59

5.1 .1 Execution of C Major Scale . . . . . . . . . . . . 60

5.1.2 Hypotheses - C Major Scale . . . . . . . . . . . 62

5.1.3 EMG Results of C Major Scale . . . . . . . . . . . . 63

5.2 Discussion . . . . . . . . . . . . . . . . 72 
5.2 .1 Execution of C Major Scale $\ldots \ldots \ldots \ldots 72$

5.2.2 EMG Results and Hypothesis SC-LO . . . . . . . . . . 73

5.2.3 EMG Results and Hypothesis SC-NC . . . . . . . . . . 74

5.3 Outcomes of Hypothesis Testing . . . . . . . . . . . . 76

6 Task \#2: Results and Discussion $\quad 77$

6.1 Results of Task \#2: Triad Exercises . . . . . . . . . . . . 77

6.1.1 Execution of Triad Exercises . . . . . . . . . . . 78

6.1.2 Hypotheses - Triad Exercises . . . . . . . . . . . . . 80

6.1.3 EMG Results of Triad Exercises . . . . . . . . . . . . . . 81

6.2 Discussion . . . . . . . . . . . . . . . . . . 90

6.2 .1 Execution of Triad Exercises _ . . . . . . . . . 90

6.2.2 EMG Results and Hypothesis TR-SC . . . . . . . . . . . 91

6.2.3 EMG Results and Hypothesis TR-HI . . . . . . . . . . . 93

6.3 Outcomes of Hypothesis Testing . . . . . . . . . . . . . . . 94

7 Task \#3: Results and Discussion $\quad 96$

7.1 Results of Task \#3: Piece by Ann Southam . . . . . . . . . . 96

7.1.1 Execution of the Piece by Ann Southam . . . . . . . 98

7.1.2 Hypotheses — Piece by Ann Southam . . . . . . . . . . 111

7.1.3 EMG results of the piece by Ann Southam . . . . . . . . . 112

7.2 Discussion . . . . . . . . . . . . . . . . . . . 121

7.2.1 Execution of Piece by Ann Southam . . . . . . . . . 121

7.2.2 EMG Results and Hypothesis AS-SC . . . . . . . . . 122

7.2 .3 EMG Results and Hypothesis AS-BE . . . . . . . . . . 123

7.3 Outcomes of Hypothesis Testing . . . . . . . . . . . . . . . 124 
8 Summary and Discussion $\quad 126$

8.1 Summary of Results . . . . . . . . . . . . . . . . . 127

8.2 Discussion of All Tasks . . . . . . . . . . . . . . . 139

8.2 .1 Normalization . . . . . . . . . . . . . . . 140

8.3 Evaluation of Study Design _. . . . . . . . . . . . . . 141

8.3.1 Measurements ..................... 142

8.3.2 Co-contraction Measurement . . . . . . . . . . 143

9 Conclusions, Contributions and Recommendations $\quad 144$

9.1 Experimental Study Conclusions . . . . . . . . . . . . . . . . 144

9.2 Contributions . . . . . . . . . . . . . . . . . 148

9.3 Recommendations for Future Study . . . . . . . . . . . . . . . . . 148

$\begin{array}{ll}\text { List of References } & 150\end{array}$

$\begin{array}{ll}\text { Appendix A Anatomical Terminology } & 156\end{array}$

Appendix B Letter of Information, Consent Form and Questionnaire

$\begin{array}{ll}\text { for Participants } & 160\end{array}$

Appendix C Code Listing 1: LoadData algorithm $\quad 166$

Appendix D Code Listing 2: Code for functions used in LoadData $\begin{array}{ll}\text { algorithm } & 171\end{array}$

Appendix E Code Listing 3: MATLAB commands to remove notes played by left hand in Ann Southam piece from MIDI data $\quad 173$

Appendix F Code Listing 4: TrimData algorithm 176 
Appendix H EMG Results of All Participants while Playing the C Major Scale in Parallel Motion

Appendix I Participants' Mean Active Muscle Stiffnesses and Mean Co-contraction Levels for Pooled Ascending and Pooled Descending Segments of the C Major Scale

Appendix J Participants' Mean Active Muscle Stiffnesses and Mean Co-contraction Levels for Ascending (A1, A2, A3) and Descending (D1, D2, D3) Segments of the C Major Scale

Appendix K Plots of Participants' Active Muscle Stiffnesses and MIDI Data While Playing Triad Exercises TR1 and TR2

Appendix L Plots of Participants' Co-contraction Levels and MIDI Data While Playing Triad Exercises TR1 and TR2

Appendix M P-values from Student's t-test comparing the mTRs to mTRe of TR1 and TR2

Appendix N Average Key Velocity in Sections of Ann Southam Piece248

Appendix O Plots of Participants' Active Muscle Stiffnesses and MIDI Data While Playing the Piece Composed by Ann Southam

Appendix P Plots of Participants' Co-contraction Levels and MIDI Data While Playing the Piece Composed by Ann Southam

Appendix Q Participants' Mean Active Muscle Stiffnesses and Mean 
Co-contraction Levels for Sections A, A', B, B', W, W', C96 and G96 of the Piece Composed by Ann Southam 


\section{List of Tables}

5.1 Participants' mean note rates of the $\mathrm{C}$ major scale expressed in notes per second ................................ 61

5.2 Summary of one-way ANOVA tests comparing mean active muscle stiffness and co-contraction levels in ascending and descending segments of the C major scale . . . . . . . . . . . . . . . . 68

5.3 Summary of pairwise comparisons of mean active muscle stiffness and co-contraction levels in $\mathrm{C}$ major scale segments $A 1, A 2$ and $A 3$ using the Tukey-Kramer method . . . . . . . . . . . . . . . 70

5.4 Summary of pairwise comparisons of mean active muscle stiffness and co-contraction levels in C major scale segments $D 1, D 2$ and $D 3$ using the Tukey-Kramer method . . . . . . . . . . . . . . . . 7

6.1 Mean duration of alternating notes in TR1 and TR2, and associated strategy used to play alternating notes . . . . . . . . . . . 80

6.2 Mean active stiffnesses in segments 2 (mTRs) and 4 (mTRe) of TR1 and $\mathrm{TR} 2 \ldots \ldots \ldots \ldots \ldots \ldots$

6.3 Mean co-contraction levels in segments 2 (mTRs) and 4 (mTRe) of

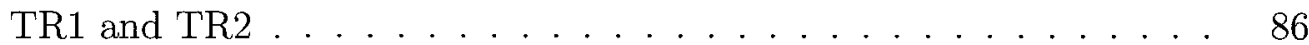

6.4 Summary of the results from Tables 6.2 and $6.3 \ldots \ldots$. . . . 87 
6.5 Summary of the results from Tables 6.2 and 6.3, divided according to the strategy used to play the alternating notes in TR1 and TR2 . .

7.1 Tally of participants' note accuracy errors made in the first attempt of the Ann Southam piece . . . . . . . . . . . . . . . . . 100

7.2 Tally of participants' note accuracy errors made in the second attempt of the Ann Southam piece. . . . . . . . . . . . . . . . . . . 101

7.3 Bars repeated or omitted by all participants during the first and second attempts of the Ann Southam piece . . . . . . . . . . . . . . 103

7.4 MIDI key velocity ranges and associated dynamic levels (adapted from Chirp Virtual MIDI Keyboard Controller User Guide - Build 1.2) . 105

7.5 Participants' mean note rates in sections A, B, C96, G96, W, A', B' and $W^{\prime}$ of the Ann Southam piece, expressed in notes per second . . 109

7.6 Summary of one-way ANOVA tests comparing mean active muscle stiffnesses of the FCU, ECU, FDS and EDC in sections A, B, C96, G96 and $\mathrm{W}$ of the Ann Southam piece . . . . . . . . . . . 115

7.7 Summary of one-way ANOVA tests comparing mean co-contraction (CC1 and CC2) in sections A, B, C96, G96 and W of the Ann Southam

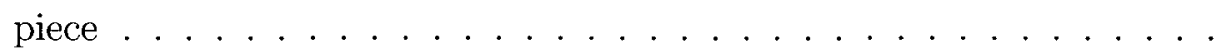

7.8 Summary of one-way ANOVA tests comparing mean active muscle stiffnesses of the FCU, ECU, FDS and EDC in sections A, B, W, A', B' and $W^{\prime}$ of the Ann Southam piece . . . . . . . . . . . . 118

7.9 Summary of one-way ANOVA tests comparing mean co-contraction (CC1 and CC2) in sections A, B, W, A', B' and W' of the Ann Southam

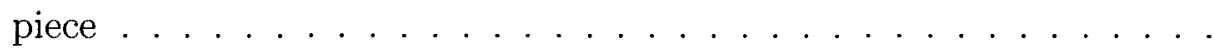

I.1 Mean active muscle stiffnesses for pooled ascending and pooled descending segments of the $\mathrm{C}$ major scale $\ldots \ldots \ldots \ldots \ldots$ 
I.2 Mean co-contraction levels for pooled ascending and pooled descending segments of the $\mathrm{C}$ major scale . . . . . . . . . . . . . . 202

J.1 Mean active muscle stiffnesses in segments $A 1, A 2$ and $A 3$ of the $\mathrm{C}$ major scale ... . . . . . . . . . . . . . . 204

J.2 Mean active muscle stiffnesses in segments $D 1, D 2$, and $D 3$ of the C major scale ........................ 205

J.3 Mean co-contraction levels in segments $A 1, A 2$ and $A 3$ of the C major

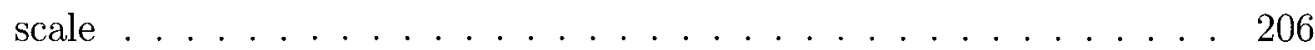

J.4 Mean co-contraction levels in segments D1, D2, and D3 of the C major

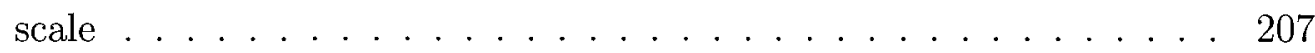

M.1 Resulting P-values from Student's t-tests comparing the mean start and end EMG values (comparing mTRs to mTRe) for both triad exercises. . . . . . . . . . . . . . . . . 247

Q.1 Mean active muscle stiffnesses of the FCU, ECU, FDS and EDC in sections A and A' of the Ann Southam piece . . . . . . . . . . 293

Q.2 Mean active muscle stiffnesses of the FCU, ECU, FDS and EDC in sections B and B' of the Ann Southam piece . . . . . . . . . . . . 294

Q.3 Mean active muscle stiffnesses of the FCU, ECU, FDS and EDC in sections $W$ and $W^{\prime}$ of the Ann Southam piece . . . . . . . . . 295

Q.4 Mean active muscle stiffnesses of the FCU, ECU, FDS and EDC in sections C96 and G96 of the Ann Southam piece . . . . . . . . 296

Q.5 Mean co-contraction levels (CC1 and CC2) in sections A and A' of the Ann Southam piece . . . . . . . . . . . . . . . 297

Q.6 Mean co-contraction levels (CC1 and CC2) in sections B and B' of the Ann Southam piece . . . . . . . . . . . . . . . . 298 
Q.7 Mean co-contraction levels (CC1 and CC2) in sections $\mathrm{W}$ and $\mathrm{W}^{\prime}$ of the Ann Southam piece . . . . . . . . . . . . . . 299

Q.8 Mean co-contraction levels (CC1 and CC2) in sections C96 and G96 of the Ann Southam piece . . . . . . . . . . . 300 


\section{List of Figures}

2.1 Skeletal muscle structural organization . . . . . . . . . . . 7

2.2 Arrangement of T-tubules and terminal cisternae . . . . . . . . 8

2.3 Sarcomere length and associated muscle tension as a percentage of the total muscle tension . . . . . . . . . . . . . . . . . 11

2.4 Front view of the skeletal structure of the lower half of the upper limb. 13

2.5 Extrinsic flexor muscles of the hand . . . . . . . . . . . . . 16

2.6 Extrinsic extensor muscles of the hand . . . . . . . . . . . 17

2.7 Force-length relationships of the gastrocnemius and sartorius muscles 20

2.8 Estimated stiffness ellipses for different hand positions of subjects A, B, C and D in Mussa-Ivaldi et al.'s 1985 study . . . . . . . . . . . . 24

2.9 Estimated inertia ellipses, stiffness ellipses and viscosity ellipses of several hand positions for all four participants in Tsuji et al.'s 1995 study. 28

2.10 Co-contraction index of the vastus lateralis and hamstrings from Unnithan et al.'s study . . . . . . . . . . . . . . 35

3.1 Bagnoli $^{\top M}$ 8-ch desktop EMG system, DAQ device and MIDI relay. . . 43

3.2 Delsys ${ }^{\circledR}$ DE 2.1 single differential sensor with dimensions. . . . . . . 43

3.3 Setup of MIDI relay, showing connections to DAQ device and Disklavier piano, and plot of resulting voltage change during G4 note on, note off $\ldots \ldots \ldots \ldots \ldots$ 
3.4 Placement of reference electrode, EDC, ECU, FDS and FCU sensors .

4.1 Summary of the algorithms used to analyze the EMG and MIDI data of the Ann Southam piece ................ . . 5

4.2 Unshifted voltage data as detected by channel 8 of the DAQ device and the resulting voltage change due to the depression of G4, corresponding MIDI data and visual representation of $\mathrm{T}$ - the time delay between the start of the EMG and MIDI date ............... 56

5.1 Participant E002's active muscle stiffness and MIDI results while playing the $\mathrm{C}$ major scale . . . . . . . . . . . . . . . . 64

5.2 Participant E002's co-contraction and MIDI results while playing the C major scale . . . . . . . . . . . . . . . . . 65

6.1 Musical score for (a) TR1 and (b) TR2 . . . . . . . . . . . 78

6.2 (a) Participant E002's active muscle stiffness and MIDI results while playing TR2. (b) Participant E002's co-contraction and MIDI results while playing TR2 . . . . . . . . . . . . . .

6.3 Example of divisions in TR1 and TR2 files showing discarded and compared segments . . . . . . . . . . . . . . .

6.4 Participant E008's active muscle stiffness and MIDI results while playing TR1(a) and TR2(b). The large increases in active muscle stiffness at the end of TR1 and TR2 are highlighted . . . . . . . . . 83

7.1 Musical score of piece composed by Ann Southam . . . . . . . . . . 97

7.2 Participant E003's mean key velocities of sections A, B, C96, G96, W, $A^{\prime}, B^{\prime}$ and $W^{\prime}$ in the Ann Southam piece . . . . . . . . . . . 107

7.3 Participant E006's mean key velocities of sections A, B, C96, G96, W, A', B' and $W^{\prime}$ in the Ann Southam piece . . . . . . . . . . . . . . 108 
7.4 Participant E002's results from the first attempt of the Ann Southam piece. Active muscle stiffness and corresponding MIDI data are shown in (a). Co-contraction and corresponding MIDI data are shown in (b).

8.1 Summary plot of participant E002's mean active muscle stiffness and

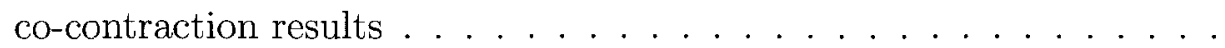

8.2 Summary plot of participant E003's mean active muscle stiffness and co-contraction results

8.3 Summary plot of participant E004's mean active muscle stiffness and

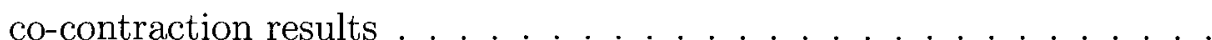

8.4 Summary plot of participant E005's mean active muscle stiffness and

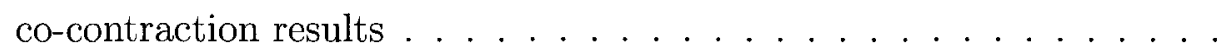

8.5 Summary plot of participant E006's mean active muscle stiffness and co-contraction results

8.6 Summary plot of participant E007's mean active muscle stiffness and co-contraction results

8.7 Summary plot of participant E008's mean active muscle stiffness and co-contraction results . . . . . . . . . . . . . . . 135

8.8 Summary plot of participant E009's mean active muscle stiffness and

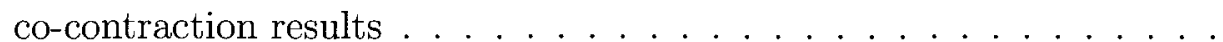

8.9 Summary plot of participant E010's mean active muscle stiffness and co-contraction results . . . . . . . . . . . . . 137

A.1 Front view of a person in the anatomical position. . . . . . . . . 157

H.1 Participant E002's active muscle stiffness and MIDI results while playing the $\mathrm{C}$ major scale . . . . . . . . . . . . . . . . . . . . 184

H.2 Participant E003's active muscle stiffness and MIDI results while playing the $\mathrm{C}$ major scale .................. 185 
H.3 Participant E004's active muscle stiffness and MIDI results while playing the C major scale . . . . . . . . . . . . . . . . . 186

H.4 Participant E005's active muscle stiffness and MIDI results while playing the $\mathrm{C}$ major scale . . . . . . . . . . . . . . 187

H.5 Participant E006's active muscle stiffness and MIDI results while playing the $\mathrm{C}$ major scale . . . . . . . . . . . . . . . . 188

H.6 Participant E007's active muscle stiffness and MIDI results while playing the $\mathrm{C}$ major scale . . . . . . . . . . . . . . . . . . 189

H.7 Participant E009's active muscle stiffness and MIDI results while playing the $\mathrm{C}$ major scale . . . . . . . . . . . . . . .

H.8 Participant E010's active muscle stiffness and MIDI results while playing the C major scale . . . . . . . . . . . . . . . . 191

H.9 Participant E002's co-contraction and MIDI results while playing the C major scale ........................

H.10 Participant E003's co-contraction and MIDI results while playing the C major scale . . . . . . . . . . . . . . . . . . 193

H.11 Participant E004's co-contraction and MIDI results while playing the C major scale . . . . . . . . . . . . . . . . . . . . 194

H.12 Participant E005's co-contraction and MIDI results while playing the C major scale . . . . . . . . . . . . . . . . . . 195

H.13 Participant E006's co-contraction and MIDI results while playing the C major scale ...................... 196

H.14 Participant E007's co-contraction and MIDI results while playing the C major scale . . . . . . . . . . . . . . . . . . . . . 197

H.15 Participant E009's co-contraction and MIDI results while playing the C major scale . . . . . . . . . . . . . . . . . 198 
H.16 Participant E010's co-contraction and MIDI results while playing the C major scale . . . . . . . . . . . . . . . . . . . 199

K.1 Participant E002's active muscle stiffness and MIDI results while play-

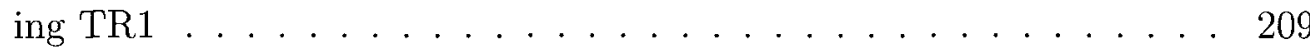

K.2 Participant E002's active muscle stiffness and MIDI results while playing $\mathrm{TR} 2 \ldots \ldots \ldots \ldots \ldots \ldots \ldots$

K.3 Participant E003's active muscle stiffness and MIDI results while play-

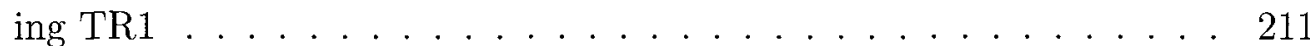

K.4 Participant E003's active muscle stiffness and MIDI results while playing TR2 . . . . . . . . . . . . . . 212

K.5 Participant E004's active muscle stiffness and MIDI results while playing $\mathrm{TR} 1 \ldots \ldots \ldots \ldots \ldots \ldots$

K.6 Participant E004's active muscle stiffness and MIDI results while playing TR2 .

K.7 Participant E005's active muscle stiffness and MIDI results while play-

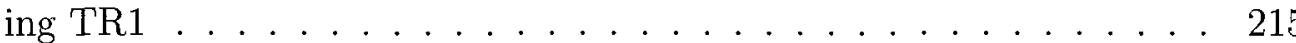

K.8 Participant E005's active muscle stiffness and MIDI results while play$\operatorname{ing} \mathrm{TR} 2 \ldots \ldots \ldots \ldots \ldots \ldots \ldots$

K.9 Participant E006's active muscle stiffness and MIDI results while playing TR1 . . . . . . . . . . . . . . . . . 217

K.10 Participant E006's active muscle stiffness and MIDI results while playing TR2 . . . . . . . . . . . . . . . 218

K.11 Participant E007's active muscle stiffness and MIDI results while play-

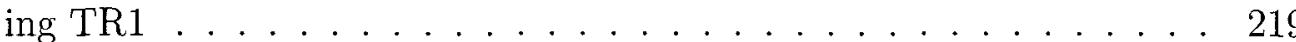

K.12 Participant E007's active muscle stiffness and MIDI results while playing TR2 
K.13 Participant E008's active muscle stiffness and MIDI results while play-

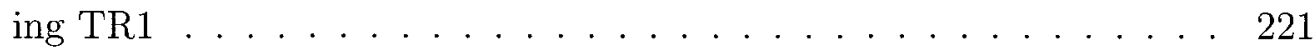

K.14 Participant E008's active muscle stiffness and MIDI results while play-

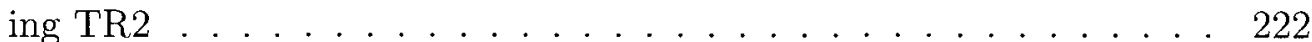

K.15 Participant E009's active muscle stiffness and MIDI results while play-

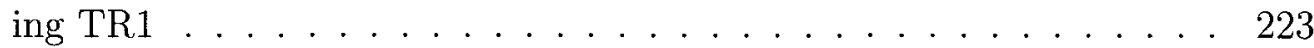

K.16 Participant E009's active muscle stiffness and MIDI results while play-

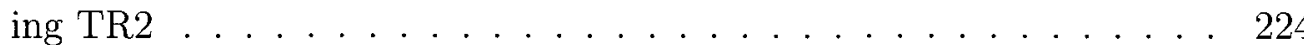

K.17 Participant E010's active muscle stiffness and MIDI results while play-

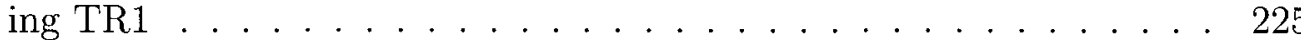

K.18 Participant E010's active muscle stiffness and MIDI results while play-

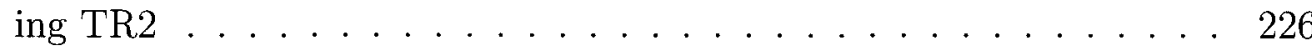

L.1 Participant E002's co-contraction and MIDI results while playing TR1 228

L.2 Participant E002's co-contraction and MIDI results while playing TR2 229

L.3 Participant E003's co-contraction and MIDI results while playing TR1 230

L.4 Participant E003's co-contraction and MIDI results while playing TR2 231

L.5 Participant E004's co-contraction and MIDI results while playing TR1 232

L.6 Participant E004's co-contraction and MIDI results while playing TR2 233

L.7 Participant E005's co-contraction and MIDI results while playing TR1 234

L.8 Participant E005's co-contraction and MIDI results while playing TR2 235

L.9 Participant E006's co-contraction and MIDI results while playing TR1 236

L.10 Participant E006's co-contraction and MIDI results while playing TR2 237

L.11 Participant E007's co-contraction and MIDI results while playing TR1 238

L.12 Participant E007's co-contraction and MIDI results while playing TR2 239

L.13 Participant E008's co-contraction and MIDI results while playing TR1 240

L.14 Participant E008's co-contraction and MIDI results while playing TR2 241 
L.15 Participant E009's co-contraction and MIDI results while playing TR1 242

L.16 Participant E009's co-contraction and MIDI results while playing TR2 243

L.17 Participant E010's co-contraction and MIDI results while playing TR1 244

L.18 Participant E010's co-contraction and MIDI results while playing TR2 245

N.1 Participant E002's mean key velocities of sections A, B, C96, G96, W, A', B' and $W^{\prime}$ in the Ann Southam piece . . . . . . . . . . . . . 249

N.2 Participant E003's mean key velocities of sections A, B, C96, G96, W, A', B' and W' in the Ann Southam piece . . . . . . . . . . . . . . 250

N.3 Participant E004's mean key velocities of sections A, B, C96, G96, W, $\mathrm{A}^{\prime}, \mathrm{B}^{\prime}$ and $\mathrm{W}^{\prime}$ in the Ann Southam piece . . . . . . . . . . . . 250

N.4 Participant E005's mean key velocities of sections A, B, C96, G96, W, A', B' and W' in the Ann Southam piece . . . . . . . . . . . . . 251

N.5 Participant E006's mean key velocities of sections A, B, C96, G96, W, A', B' and $W^{\prime}$ in the Ann Southam piece . . . . . . . . . . . . . . 251

N.6 Participant E007's mean key velocities of sections A, B, C96, G96, W, A', B' and $W^{\prime}$ in the Ann Southam piece . . . . . . . . . . . . . 252

N.7 Participant E008's mean key velocities of sections A, B, C90 (attempt 1), C96 (attempt 2), G96, W, A', B' and W' in the Ann Southam piece 252

N.8 Participant E009's mean key velocities of sections A, B, C96, G96, W, A', B' and W' in the Ann Southam piece . . . . . . . . . . . . 253

N.9 Participant E010's mean key velocities of sections A, B, C73 (attempt 1), C96 (attempt 2), G96, W, A', B' and W' in the Ann Southam piece 253

O.1 Participant E002's active muscle stiffness and MIDI results while playing the Ann Southam piece for the first time . . . . . . . . . 255

O.2 Participant E002's active muscle stiffness and MIDI results while playing the Ann Southam piece for the second time . . . . . . . . . 256 
O.3 Participant E003's active muscle stiffness and MIDI results while playing the Ann Southam piece for the first time . . . . . . . 257

O.4 Participant E003's active muscle stiffness and MIDI results while playing the Ann Southam piece for the second time . . . . . . . . 258

O.5 Participant E004's active muscle stiffness and MIDI results while playing the Ann Southam piece for the first time . . . . . . . . . 259

O.6 Participant E004's active muscle stiffness and MIDI results while playing the Ann Southam piece for the second time . . . . . . . . 260

O.7 Participant E005's active muscle stiffness and MIDI results while playing the Ann Southam piece for the first time . . . . . . . . . 261

O.8 Participant E005's active muscle stiffness and MIDI results while playing the Ann Southam piece for the second time . . . . . . . . 262

O.9 Participant E006's active muscle stiffness and MIDI results while playing the Ann Southam piece for the first time . . . . . . . . 263

O.10 Participant E006's active muscle stiffness and MIDI results while playing the Ann Southam piece for the second time . . . . . . . . . 264

O.11 Participant E007's active muscle stiffness and MIDI results while playing the Ann Southam piece for the first time . . . . . . . . . .

O.12 Participant E007's active muscle stiffness and MIDI results while playing the Ann Southam piece for the second time . . . . . . . 266

O.13 Participant E008's active muscle stiffness and MIDI results while playing the Ann Southam piece for the first time . . . . . . . . . 267

O.14 Participant E008's active muscle stiffness and MIDI results while playing the Ann Southam piece for the second time . . . . . . . 268

O.15 Participant E009's active muscle stiffness and MIDI results while playing the Ann Southam piece for the first time . . . . . . . . . 269 
O.16 Participant E009's active muscle stiffness and MIDI results while playing the Ann Southam piece for the second time . . . . . . 270

O.17 Participant E010's active muscle stiffness and MIDI results while playing the Ann Southam piece for the first time . . . . . . . . . 271

O.18 Participant E010's active muscle stiffness and MIDI results while playing the Ann Southam piece for the second time $\ldots \ldots \ldots . . \ldots 272$

P.1 Participant E002's co-contraction and MIDI results while playing the Ann Southam piece for the first time . . . . . . . . . . 274

P.2 Participant E002's co-contraction and MIDI results while playing the Ann Southam piece for the second time . . . . . . . . . . 275

P.3 Participant E003's co-contraction and MIDI results while playing the Ann Southam piece for the first time . . . . . . . . . 276

P.4 Participant E003's co-contraction and MIDI results while playing the Ann Southam piece for the second time . . . . . . . . . 277

P.5 Participant E004's co-contraction and MIDI results while playing the Ann Southam piece for the first time . . . . . . . . . 278

P.6 Participant E004's co-contraction and MIDI results while playing the Ann Southam piece for the second time . . . . . . . . . . 279

P.7 Participant E005's co-contraction and MIDI results while playing the Ann Southam piece for the first time . . . . . . . . . . 280

P.8 Participant E005's co-contraction and MIDI results while playing the Ann Southam piece for the second time . . . . . . . . . . . . 281

P.9 Participant E006's co-contraction and MIDI results while playing the Ann Southam piece for the first time . . . . . . . . . . . 282

P.10 Participant E006's co-contraction and MIDI results while playing the Ann Southam piece for the second time . . . . . . . . . . . 283 
P.11 Participant E007's co-contraction and MIDI results while playing the Ann Southam piece for the first time . . . . . . . . . . . . . . 284

P.12 Participant E007's co-contraction and MIDI results while playing the Ann Southam piece for the second time . . . . . . . . . . . . 285

P.13 Participant E008's co-contraction and MIDI results while playing the Ann Southam piece for the first time . . . . . . . . . . 286

P.14 Participant E008's co-contraction and MIDI results while playing the Ann Southam piece for the second time . . . . . . . . . . . . . 287

P.15 Participant E009's co-contraction and MIDI results while playing the Ann Southam piece for the first time . . . . . . . . . . . 288

P.16 Participant E009's co-contraction and MIDI results while playing the Ann Southam piece for the second time . . . . . . . . . . . . 289

P.17 Participant E010's co-contraction and MIDI results while playing the Ann Southam piece for the first time . . . . . . . . . . 290

P.18 Participant E010's co-contraction and MIDI results while playing the Ann Southam piece for the second time . . . . . . . . . . . . . . . 291 


\section{List of Abbreviations}

This is a list of the abbreviations used throughout this thesis:

$\alpha-\mathrm{mn} \quad$ Alpha motoneuron

ANOVA Analysis of variance

bpm Beats per minute

CC1 Co-contraction of a flexor and extensor having their origin in the forearm and where their primary action is at the wrist

CC2 Co-contraction of a flexor and extensor having their origin in the forearm and where they have a secondary action at the wrist

CCM Co-contraction magnitude

CCR Co-contraction ratio

CI Co-contraction index

DAQ Data acquisition

Continued on next page 
DIP distal interphalangeal [joint]

ECU Extensor carpi ulnaris [muscle]

EDC Extensor digitorum communis [muscle]

EMG Electromyography

fr1 Finger 1, more commonly known as the thumb

fr2 Finger 2, more commonly known as the index finger

fr3 Finger 3, more commonly known as the middle finger

fr4 Finger 4, more commonly known as the ring finger

fr5 Finger 5, more commonly known as the pinky or baby finger

FCR Flexor carpi radialis [muscle]

FCU Flexor carpi ulnaris [muscle]

FDP Flexor digitorum profundus [muscle]

FDS Flexor digitorum superficialis [muscle]

FPL Flexor profundus longus [muscle]

HPF High pass filter

Continued on next page 
IMCJ Index of muscle co-contraction around the joint

IP Interphalangeal [joint]

LE Linear envelope

LPF Low pass filter

MCP Metacarpophalangeal [joint]

MES Myoelectric signal

MG Medial gastrocnemius [muscle]

MIDI Musical instrument digital interface

mTRe Mean of end or segment $4(\approx 3 \mathrm{~s})$ of triad exercise

mTRs Mean of start or segment $2(\approx 3 \mathrm{~s})$ of triad exercise

MUAP Motor unit action potential

MVC Maximal voluntary contraction

Mykin Myokinetic

PFM Parallel link drive air-magnet floating manipulandum

PIP Proximal interphalangeal [joint] 
PPM Pearson Product-Moment

RCI Reciprocal contraction index

RDS Relative difference signal

RMS Root mean squared

SOL Gastrocnemius and soleus [muscles]

SysEx System exclusive [message]

TA Tibialis anterior [muscle]

TR1 First triad exercise

TR2 Second triad exercise 


\section{Chapter 1}

\section{Introduction}

\subsection{Motivation}

Playing the piano is a fairly common activity and yet the biomechanics underlying piano playing are still not well understood. Often, piano teachers have too little background in biomechanics and physiology to understand what is involved in playing the piano [1]; students generally have even less background in these fields than their teachers. Because playing the piano is a complex task, creative approaches to pedagogy are used, and they commonly involve metaphors. Metaphors are often used by teachers to communicate to their students a means of achieving a certain movement (e.g. Fink [2]). Because of the necessary impreciseness of these metaphors, there can be inconsistencies in the messages they convey, even within one pedagogical school. Furthermore, in teaching, biomechanical terms are often used to describe piano technique, even though these terms are used ambiguously and without the teacher or

the student having complete understanding of their meaning [2]. Four interrelated biomechanical concepts that are used differently by pedagogues and scientists have been identified: stıffness, relaxatıon, co-contractıon and multı-joınt ıssues [3].

Joint stiffness arises from the agonist-antagonist muscle pair that moves the joint. 
Muscles exist in pairs because they can only pull; they cannot push [4]. The agonist generates a movement in one direction, and the antagonist generates a movement in the opposite direction. When both the agonist and antagonist pull equally, the joint will not move, and the stiffness of the joint increases. The simultaneous contraction of the agonist and antagonist is called co-contraction. This relationship between joint stiffness and co-contraction allows co-contraction to serve as an indirect indicator of joint stiffness. Co-contraction yields only an estimate of joint stiffness because there are other factors, such as passive muscle and ligament properties, that contribute to joint stiffness. There is misconception among prominent members of the music community that stiffness and co-contraction are injurious. For example Mark [5] states that, "Co-contraction inhibits movement and can cause injury." He is not alone. In one of her video volumes, Taubman [6] states that co-contraction (which she refers to as a "dual-muscular pull") should be avoided. Fraser [7] refers to the "evils of co-contraction" and "useless co-contraction," representing co-contraction as being negative.

\subsection{Problem Statement}

The overall goal was to investigate active muscle stiffness and co-contraction in the hands and wrists of participants who played several exercises on the piano. The results will be compared to the results of Vant [3], who found no statistically significant change in impedance at the wrist in participants who played a piece composed for her study.

Depressing keys on the piano calls upon the finger flexor muscles, but to maintain proper arm posture wrist extensor muscles must contract to prevent the wrist from collapsing. This illustrates from a physiological point of view that co-contraction 
may be essential for piano playing. One of the goals of the study is to quantify levels of co-contraction of advanced piano players $^{1}$ during piano playing. Specifically, the co-contraction of muscles acting at and crossing the wrist will be examined.

Disorders of the forearms and wrists are most common in piano students, with $22 \%$ of students affected [8]. By better understanding co-contraction in muscles acting at the wrist and the biomechanics of piano playing, perhaps causes of pain and injury can be better understood.

In this study, subjects will be asked to perform three distinct playing tasks: a scale, two triad exercises for finger independence, and a piece of music chosen by the researchers. While the subject is playing, surface electromyography (sEMG) data will be recorded from electrodes placed on the subject's forearm. Analysis of sEMG data will reveal the levels of contraction in muscles of the forearm, from which cocontraction will be computed. Musical Instrument Digital Interface (MIDI) ${ }^{3}$ data will be recorded from the piano.

\subsection{Outline}

The following chapter describes the anatomical background relevant to the study. This information reviews the structure of muscle, muscle contractions, the skeletal structure of the forearm and actions of the muscles of the forearm. It describes EMG and where the myoelectric signal originates. A large portion of the chapter is dedicated to clarifying the types of stiffness, specifically muscle stiffness (both

\footnotetext{
${ }^{1}$ In these experiments, an advanced piano player is someone who has received long-term piano training, meaning they have been playing for at least 15 years or have studied piano performance at the undergraduate level (or both).

${ }^{2}$ Surface electromyography is a technique for measuring an electrical signal on the surface of the body, in this case the arm, that is related to the level of muscular contraction of the underlying muscles.

${ }^{3}$ Musical Instrument Digital Interface allows communication and data exchange between instruments, computers and other equipment. It is used to record key velocity, pitch, accuracy and timing.
} 
passive and active) and joint stiffness, and how they are related. Techniques used to measure joint stiffness are reviewed, and the relationships between joint stiffness, multi-joint issues and impedance are described. The chapter then goes on to describe co-contraction, various methods of quantifying it, and studies that have measured co-contraction. The last part of this chapter describes the work of Christy Vant [3], who measured driving point impedance in response to a perturbation administered to the wrist.

The experimental protocol is described in Chapter 3. This chapter outlines the motivation for the study, the selection of participants, the equipment used, the setup and connections between pieces of equipment and the three tasks performed by the participants. The following chapter (Chapter 4) describes how EMG data were filtered, and how EMG task data were normalized. This includes explanations of column entries of the MIDI matrix, the method use to synchronize MIDI and EMG data, and the division of EMG files into sections.

The results of the three experimental tasks are presented in Chapters 5,6 and 7 . The execution of each experimental task is assessed. This is followed by the evaluation of proposed hypotheses concerning the experimental task in question. Next, the EMG results are presented, which is followed by a discussion of the results, and in some cases, new studies are proposed.

The following chapter (Chapter 8) presents a summary of the results and relates the results to each other. The second half of the chapter discusses the study as a whole and comments on the normalization techniques used, evaluates the design of the study and the measurements made.

Experimental study conclusions, contributions of the work and recommendations for future study are briefly described in the last chapter (Chapter 9), which concludes the document. 


\section{Chapter 2}

\section{Background and Literature Review}

As indicated in Chapter 1, it has been established that changes in co-contraction are a rough indication of changes in joint stiffness. To understand how co-contraction can be measured using EMG, a basic understanding of muscle microstructure and the physiology of a muscle contraction is beneficial. To this end, the following chapter describes these two elements. Since the focus of this study is on the co-contraction of muscles with a primary or secondary action at the wrist, the chapter proceeds to detail the musculoskeletal structures of the forearm. The chapter also provides a brief overview of EMG and the origin of the myoelectric signal.

Building on those basic concepts, the chapter goes on to explore the topic of stiffness. It describes different types of stiffnesses, with a heavy emphasis on joint stiffness, and how it is measured. It then explores the topic of co-contraction, the various ways is has been computed, and highlights some of the findings of earlier studies that measured co-contraction in activities other than piano playing and their

relevance. This chapter concludes with a summary of the approach used by Vant [3] in her 2007 study, where she used force perturbations applied to the wrist to observe changes in driving point impedance. 


\subsection{Anatomy and Physiology Background}

This section reviews the structure of muscles, how they contract, and some of the musculoskeletal structures used to play the piano.

\subsubsection{Muscles - Structure and Function}

The musculoskeletal system serves two main functions: mobility and stability. Muscle contractions produce movement or locomotion by moving bones around a joint. Muscles play a role in joint stabilization by contracting to maintain body posture or position [9].

\section{Muscle Macro- and Microstructure}

There are three types of muscle tissues in the human body; however, only the structure and function of skeletal muscle tissue will be discussed.

Skeletal muscle, simply referred to as muscle throughout this document, is wrapped in layers of connective tissue. The outer layer, the epimysium, surrounds the muscle. The central layer, the perimysium, surrounds bundles of muscle fibres called fascicles. The perimysium contains the blood vessels and nerves that supply each fascicle. The inner layer, the endomysium, surrounds each muscle fibre. The collagen fibres of these layers of connective tissue converge at each end of the muscle forming tendons, which attach to bones, and allow muscles to stabilize or produce movement about joints [10] (see Figure. 2.1).

The bulk of skeletal muscles is made up of muscle cells, which are called muscle fibres. The structures of a muscle fibre are shown in Figure 2.1. Muscle fibres are $10 \mu \mathrm{m}$ to $100 \mu \mathrm{m}$ in diametre and can be as long as $30 \mathrm{~cm}$ [11]. A muscle fibre is typically the same length as the muscle to which it belongs, thus there is variability in 


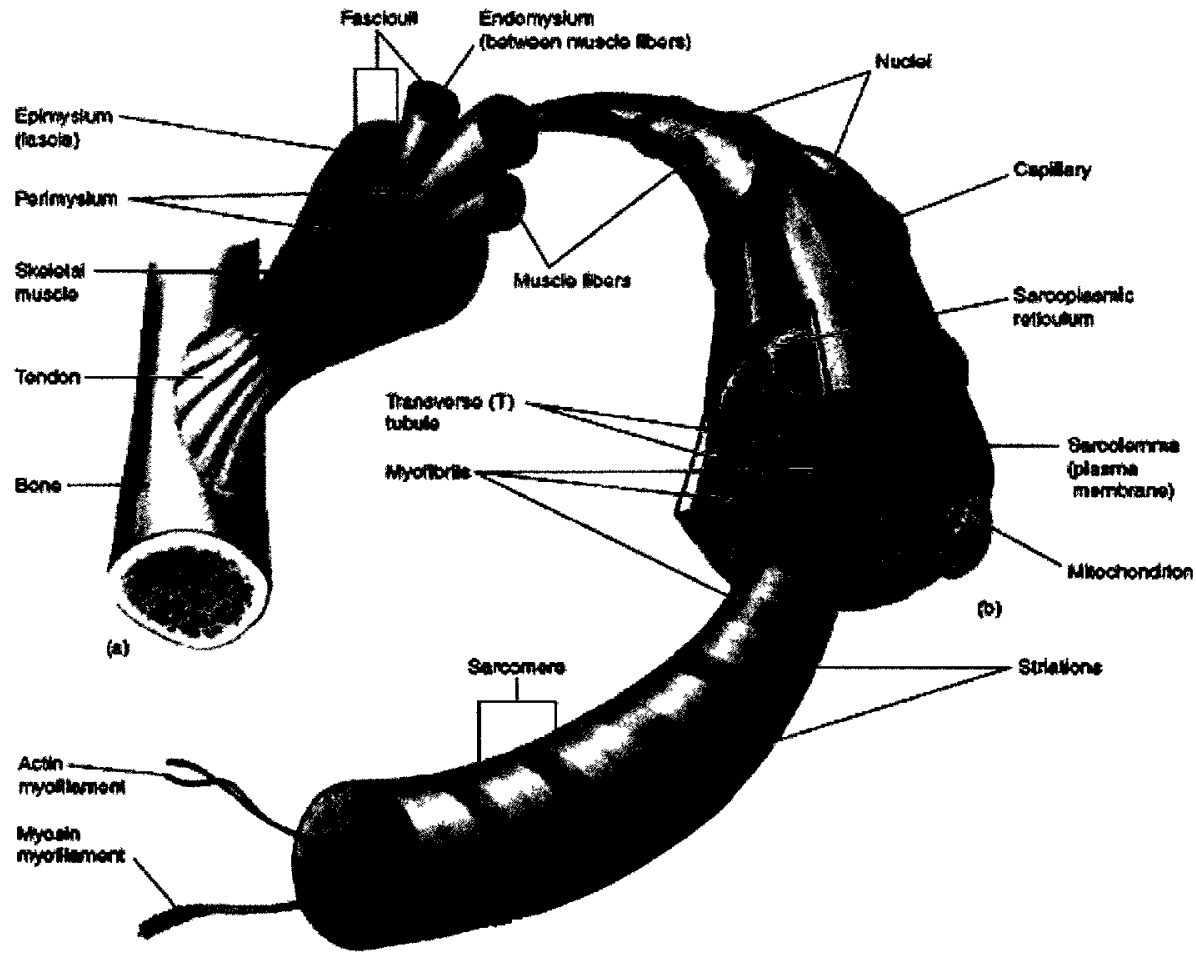

Figure 2.1: A sketch showing the structural organization of skeletal muscle, obtained from $[12]$.

the length of human muscle fibres. As with most cells, muscle fibres are surrounded by a cell membrane, the sarcolemma, and are filled with cytoplasm, the sarcoplasm. The surface of the sarcolemma contains invaginations that form a network of narrow tubules surrounding the myofibrils called transverse tubules or T-tubules. T-tubules aid in the coordination and stimulation of muscle contractions [10].

Within the sarcoplasm of each muscle fibre are hundreds to thousands of myofibrils. Myofibrils are the structures responsible for muscle contraction and thus span the length of the muscle fibre. A myofibril is made up of bundled, overlapping thick and thin protein filaments, and is encased by a membrane, the sarcoplasmic reticulum. The sarcoplasmic reticulum forms a transport network throughout the cell 


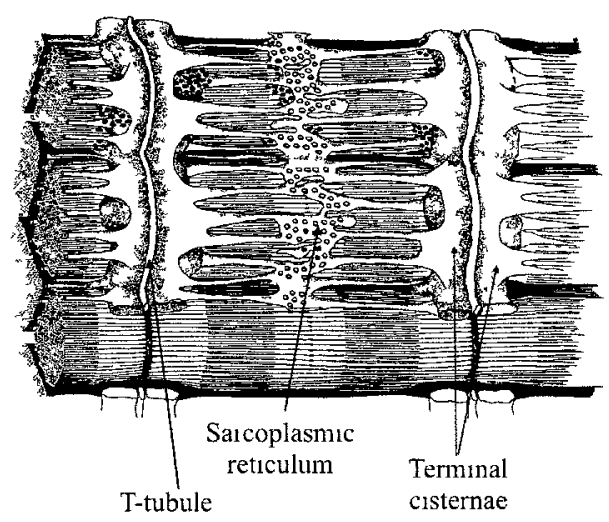

Figure 2.2: A sketch showing the arrangement of T-tubules and terminal cisternae, obtained from [13].

and houses calcium, which is necessary for muscle contractions. The tubules of the sarcoplasmic reticulum, one on either side of a T-tubule, get larger and fuse together forming chamber-like structures. These chamber-like structures are called terminal cisternae, and they are closely associated with T-tubules, although there is no direct connection between them. The organization of the T-tubules and terminal cisternae is shown in Figure 2.2.

As already mentioned, myofibrils are made up of thick and thin filaments. The thick filament is composed of the protein myosin, and the thin filament is composed primarily of the protein actin. Actin and myosin filaments are arranged into repeating units called sarcomeres. A sarcomere is the functional unit of a myofibril. The myosin filament is at the middle of the sarcomere, and the actin filaments are at the ends of the sarcomere. Actin filaments are joined to other actin filaments in adjacent sarcomeres. A schematic of one sarcomere with varying amounts of overlap of the filaments is shown in Figure. 2.3. 


\section{Muscle Contraction}

Muscle fibres are innervated by alpha motor neurons (or $\alpha$-mn). An $\alpha$-mn has its cell body in the central nervous system - either in the brainstem or in the spinal cord. The cell body receives input from interneurons (in the case of spinal reflexes) or from higher motor pathways (as in playing the piano). The axon of an $\alpha$-mn extends from its cell body to the muscle it innervates. Near the muscle the axon branches, and each branch extends to an individual muscle fibre. The $\alpha$-mn and the muscles fibres it innervates is called a motor unit. The number of muscle fibres innervated per $\alpha-\mathrm{mn}$ is called the innervation ratio and varies by muscle. Where fine motor control is required, muscles typically have a low innervation ratio (e.g. muscles of the eye); muscles with a high innervation ratio (e.g. large muscles in the leg) are responsible for gross motor control [9].

For a voluntary muscle contraction to take place, muscle fibres must receive input from the nervous system. The input originates in the central nervous system as an action potential. An action potential is triggered by a change in the cell membrane's permeability to sodium ions, which occurs in response to a stimulus, resulting in a change in the transmembrane potential. The localized change in polarity causes the adjacent part of the membrane to depolarize, and an action potential travels as a wave down the axon of the $\alpha$-mn until it reaches the axon terminal. The action potential crosses the space between the axon terminal and muscle fibre (i.e. the synaptic cleft) by a biochemical process. In the muscle fibre, the action potential spreads throughout the cell by means of the network of T-tubules. As the action potential is distributed throughout the cell by the T-tubules, it causes nearby terminal cisternae to become permeable to calcium, triggering a release of calcium into the sarcoplasm. Calcium release causes a conformational change of myofibril proteins that unblocks the binding sites on the actin protein filament. The actin and myosin protein filaments bind, 
resulting in the formation of cross-bridges [10]. The "stroke" that causes the actin and myosin filaments to slide past one another occurs when ATP is cleaved into ADP and inorganic phosphate (molecules important for energy transfer in cells) and the phosphate molecule is released from the thick filament. It results in a shortened muscle fibre and muscular contraction [11].

The amount of overlap of the actin and myosin filaments determines the amount of tension muscles are able to generate. As the amount of overlap of the contractile filaments increases or decreases beyond optimal resting length, muscles do not generate as much tension. When there is insufficient overlap of the actin and myosin filaments, cross-bridges cannot form and the muscle cannot generate tension. Similarly, when there is too much overlap of the thick and thin filaments so that actin filaments overlap, muscles do not generate as much tension. The optimal resting length refers to the length of the sarcomere at which a muscle fibre is able to generate the highest amount of tension (see Figure. 2.3).

\subsubsection{The Forearm, Wrist, Hand and Fingers}

There are many movements of the upper limb that are used while playing the piano: protraction/retraction, elevation/depression, abduction/adduction, flexion/extension of the shoulder; flexion/extension of the elbow; pronation/supination of the forearm at the radioulnar joint; flexion/extension, radial/ulnar deviation ${ }^{1}$, circumduction of the wrist; abduction/adduction, flexion/extension of the knuckles; and flexion/extension of the finger joints. The meanings of these terms of movement, as well as other terminology used in anatomical descriptions, are explained in Appendix A.

Measuring all the muscles responsible for these movements would be challenging,

\footnotetext{
${ }^{1}$ In the anatomical position, radial deviation can also be referred to as abduction of the wrist; ulnar deviation can also be referred to as adduction of the wrist.
} 


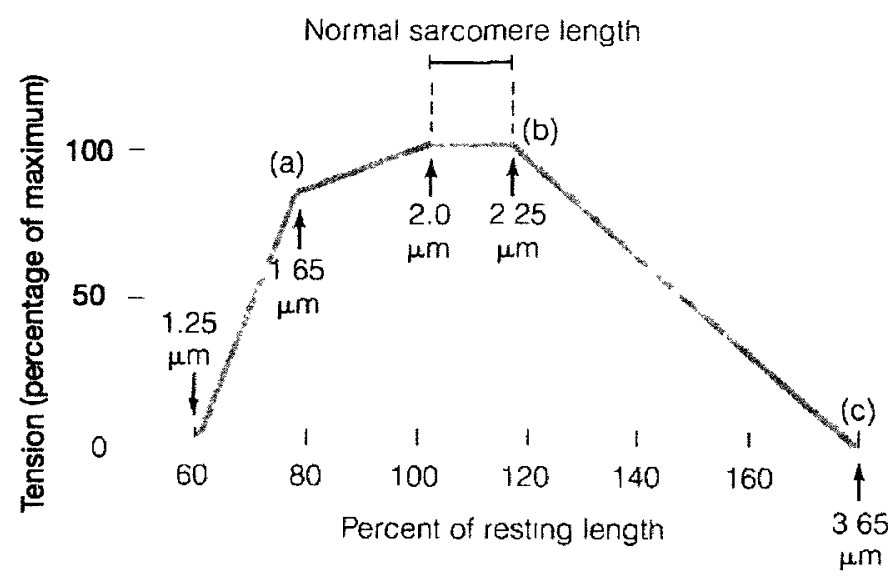

(b)

(a)
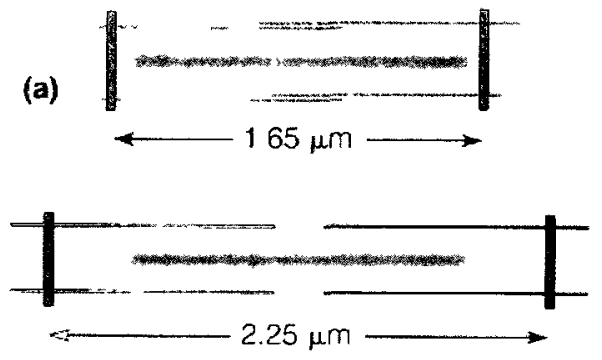

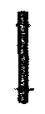

(c)

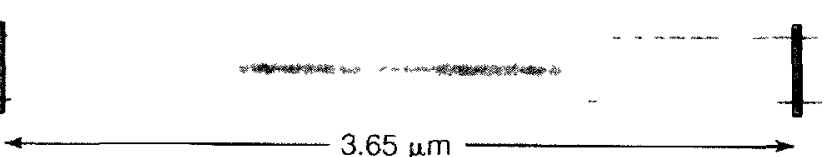

Figure 2.3: Sarcomere length and associated muscle tension as a percentage of the total muscle tension the muscle is able to generate, obtained from Marieb's Human Anatomy and Physiology [14]. In (a), there is overlap of the actin filaments, thus contraction strength (i.e. tension) is reduced. In (b) and in the yellow zone, there is optimal overlap of the actin and myosin filaments so that cross-bridges can form without the actin filaments crossing the centre of the sarcomere and overlapping. This is called optimal resting length. Maximum contraction strength is achieved when the sarcomere is at its optimal resting length. In (c), the sarcomere is stretched so that there is no overlap of the actin and myosin filaments at all, thus cross-bridges cannot form and contraction does not occur. 
and would yield huge amounts of data. This study will examine the contribution of four muscles of the lower half of the upper limb during piano playing. Two of these muscles move fingers and knuckles; all four muscles have actions (be they primary or secondary) at the wrist. The following two sections will describe the location of these muscles, and will present an overview of the anatomy of the forearm, wrist, hand and fingers relevant to this study.

\section{Skeletal Structure of the Forearm}

The forearm begins where the upper arm ends. The bone of the upper arm is the humerus. It has bony processes on its medial (or inner) and lateral (or outer) sides called the medial epicondyle and lateral epicondyle, respectively. The epicondyles serve as the point of attachment for several muscles. As a general rule, the medial epicondyle serves as a proximal point of attachment for the flexor muscles of the forearm (either partially or fully), and the lateral epicondyle serves as a point of attachment for the extensor muscles of the forearm (again, either partially or fully). The two bones of the forearm, the ulna and radius, articulate with the humerus, forming the humeroulnar joint and the humeroradial joint, respectively [10]. These joints are shown in Figure. 2.4. Collectively, the humeroulnar joint and humeroradial joint form the elbow.

Distally, the radius articulates with the carpal bones forming the radiocarpal joints. The carpal bones also articulate with each other forming gliding joints called intercarpal joints. Proximal to the radiocarpal joint, the radius articulates with the ulna forming the radioulnar joint that allows for pronation and supination of the

forearm. The wrist is made up of the radiocarpal joints, intercarpal and radioulnar joints (see Figure. 2.4. The radiocarpal joints permit ulnar and radial deviation. 


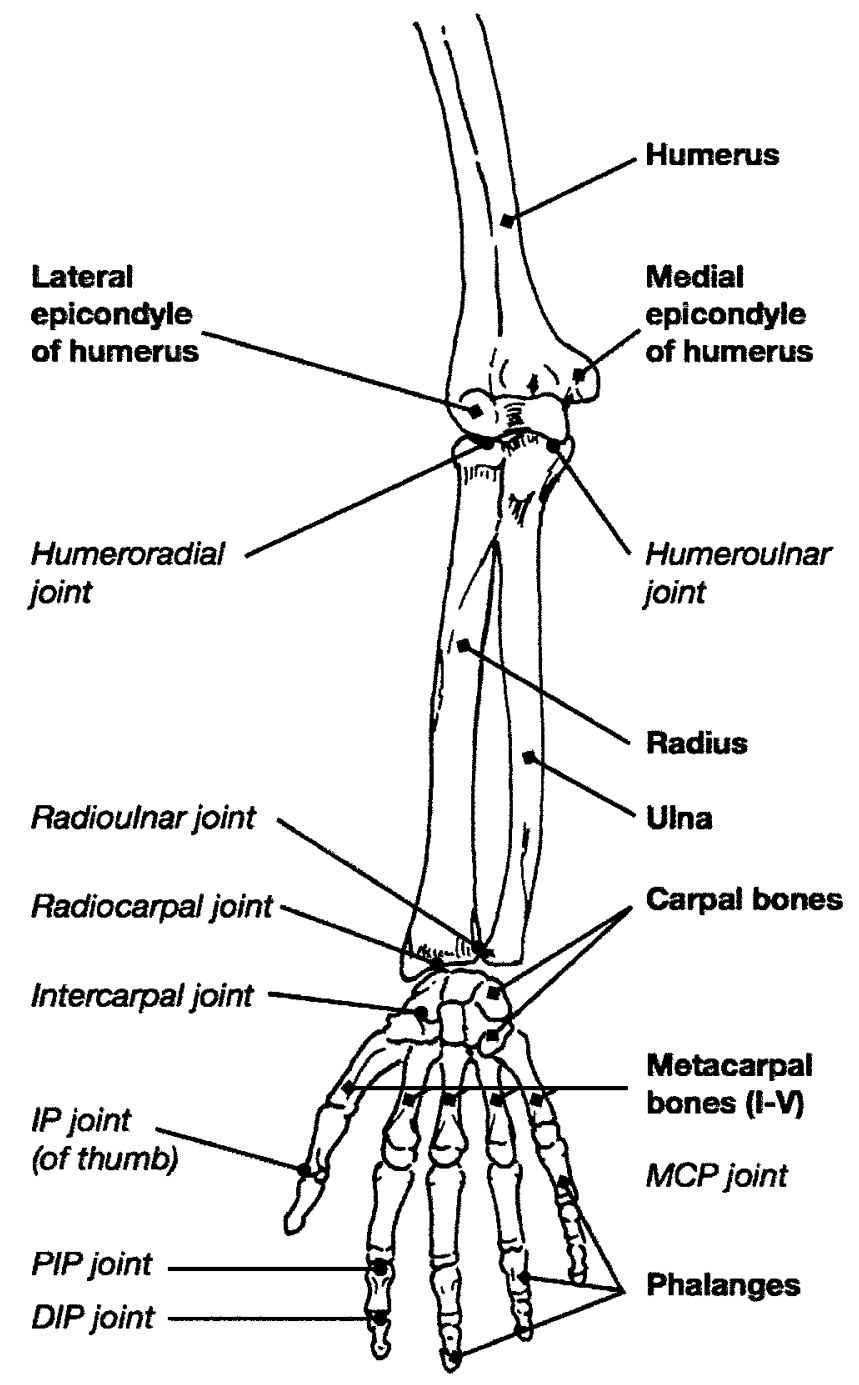

Figure 2.4: Anterior view of the skeletal structure of the lower half of the upper limb. Right arm shown. Bones are labelled in bold font; articulations between bones are labelled in italic font. Adapted from Nordin and Frankel's Basic Biomechanics of the Musculoskeletal System [15]. 
The carpal bones are arranged in two rows. The distal row articulates with the metacarpals forming the carpometacarpal (CMC) joints. The metacarpals are found in the palm of the hand. They articulate with the proximal phalanges, forming the metacarpophalangeal joints, or MCP joints. The MCP joints correspond to the knuckles. Each hand has fourteen phalanges: two in the thumb (proximal and distal) and three in each finger (proximal, middle and distal). Articulations between phalanges are referred to as interphalangeal joints, or IP joints. Where the proximal and middle phalanges articulate, the proximal interphalangeal (or PIP) joints are formed; where the middle and distal phalanges articulate, the distal interphalangeal (or DIP) joints are formed. The joint between the proximal and distal phalanges of the thumb is simply referred to as the interphalangeal joint. It is able to flex and extend, like the PIP and DIP joints. The thumb is able to perform circumduction ${ }^{2}$ and opposition ${ }^{3}$ movements because of the geometry of its CMC joint. The CMC, MCP, IP, PIP and DIP joints are shown in Figure. 2.4.

\section{Muscles of the Forearm}

While the geometry of the bones dictates the types of movements the joints can perform, it is the muscles that cause these movements to take place. Muscles have an origin (proximal point of attachment) and an insertion (distal point of attachment). Muscles can be synergistic and redundant in their actions. For instance, finger flexion is accomplished primarily by the flexor digitorum profundus and the flexor digitorum superficialis. These muscles act together synergistically to control

\footnotetext{
${ }^{2}$ Circumduction is a type of angular motion, where the body segment is fixed at one end and the other end can be moved in a complete circle. Circumduction can be described as a combination of flexion/extension and abduction/adduction.

${ }^{3}$ Opposition is a special movement that can only be accomplished by the thumbs. Opposition refers to the pad to pad contact between the thumb and any of the fingers or the thumb and the palm of the hand.
} 
finger flexion. Flexion of the PIP and MCP joints is carried out by the flexor digitorum superficialis, but the flexor digitorum profundus also flexes the PIP and MCP joints when it contracts [9], making these two muscles redundant in function.

There are multiple muscles that control wrist position. They are referred to as the extrinsic muscles of the hand, or simply the extrinsics, because they originate outside of the hand, and insert on the wrist, hand or fingers. Many of them originate on one of the epicondyles of the humerus, and several originate along the forearm. Some of these muscles insert on the carpals or metacarpals, and have their primary actions at the wrist; others insert on the phalanges, and have their primary actions at the digits or thumb, but have a secondary action at the wrist. The extrinsics are also classified according to their action: flexion or extension. There are six extrinsic flexor muscles and nine extrinsic extensor muscles.

Of the six extrinsic flexor muscles, which are shown in Figure. 2.5, three have their primary action at the wrist. These are the flexor carpi radialis (FCR), the flexor carpi ulnaris (FCU) and the palmaris longus (PL). The remaining three extrinsic flexor muscles flex the MCP and IP joints of the fingers (the flexor digitorum superficialis or FDS and the flexor digitorum profundus or FDP) and of the thumb (the flexor profundus longus or FPL). Because the FDS is farther from the MCP joint than the FDP, and because the FDS crosses fewer joints than the FDP, the FDS is able to produce more torque at the MCP joint [9].

Of the nine extrinsic extensor muscles, which are shown in Figure. 2.6, three have their primary action at the wrist, three are responsible for the extension of the IP joints of the fingers, and three extend and abduct the thumb. The extensor carpi radialis longus (ECRL), extensor carpi radialis brevis (ECRB) and extensor carpi ulnaris (ECU) have their primary action at the wrist. The extensor digitorum 

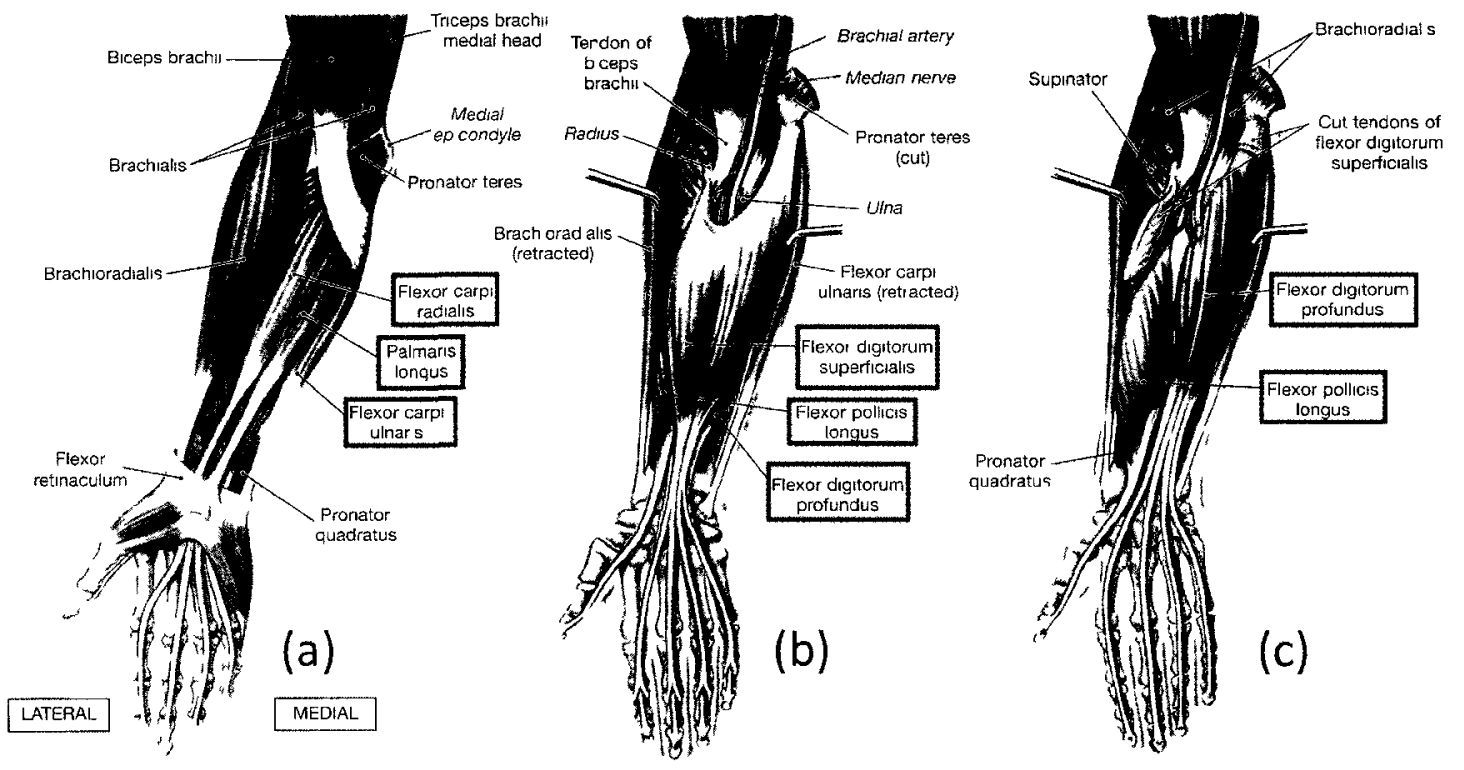

Figure 2.5: The palmar side of the hand and forearm, showing the (a) superficial, (b) middle, and (c) deep extrinsic flexor muscles. Obtained from Martini et al.'s Human Anatomy [10].

communis (EDC), extensor indicis proprius (EIP) and extensor digiti minimi (EDM) are IP joint extensors of the fingers, but cross the wrist, thereby having a secondary action at the wrist. The extensor pollicis longus (EPL) extends the MCP and IP joints of the thumb; both the extensor pollicis brevis (EPB) and the abductor pollicis longus (APL) abduct the CMC joint at the base of the thumb, but, of the two, only the EPB extends the MCP joint of the thumb. All three of these muscles controlling movements of the thumb originate in the forearm and cross the wrist before inserting on one of the metacarpals or phalanges, thus having a secondary action at the wrist [9].

This study will measure the myoelectric signal from the FCU, ECU, FDS and EDC. These muscles were selected because the FCU and ECU represent an antagonistic muscle pair with their primary actıon at the wrist; the FDS and EDC represent an antagonistic pair of muscles with a secondary action at the wrist. 


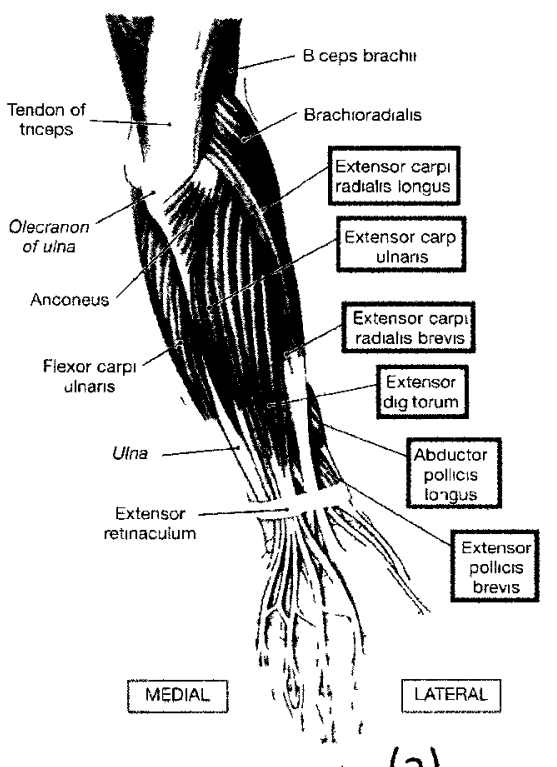

(a)

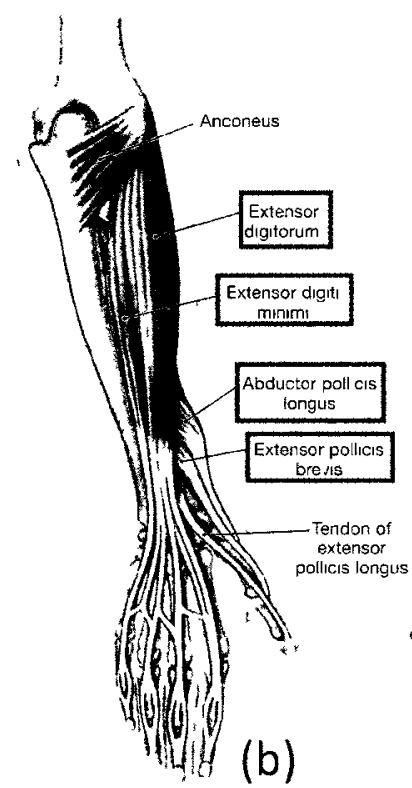

(b)

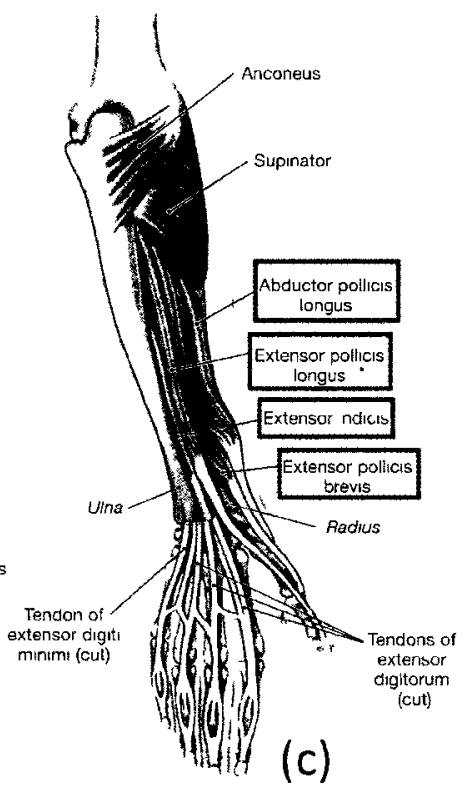

Figure 2.6: The volar side of the hand and forearm, showing the (a) superficial, (b) middle, and (c) deep extrinsic extensor muscles. Obtained from Martini et al.'s Human Anatomy [10].

\subsection{Electromyography}

Electromyography (EMG) is the study of muscle function by detecting, analyzing and using the complex electrical signal, termed the myoelectric signal or MES, muscles generate when they contract. The electrical signal arises from the ionic flow across the sarcolemma that occurs during muscle contraction. The emanating signal can be detected using conductive electrodes. The term "electromyography" is outdated. In the past, the myoelectric signal was output in "graphic" form; today it is quite common to capture the MES using electronic components, like computers. Despite this transition, the word "electromyography" continues to be used by clinicians [16].

As mentioned in Section 2.1.1, a muscle contraction is initiated when a muscle receives neural input from one or multiple $\alpha$-mn. Each axonal branch of an $\alpha$-mn innervates a single muscle fibre, and the combination of an $\alpha$-mn and the fibres it 
innervates is called a motor unit. When the muscle fibres of a motor unit are activated, the resulting electrical signal detected by an electrode is called a motor unit action potential (or MUAP). This is the fundamental unit of the MES. The summation of MUAPs is the MES.

There are two types of electrodes used in EMG: surface electrodes attached to the skin and intramuscular electrodes (either wire or needle) inserted into the muscle of interest. The MES captured by surface electrodes is a summation of MUAPs generated by the underlying muscle tissue. Intramuscular electrodes are inserted through the skin into a muscle and have a much smaller surface area. As such, they can be used to record the MES of deep muscles, and are capable of detecting individual MUAPs. The MES collected using surface EMG gives an indication of the overall activity of the muscle in question rather than the activity of a few muscle fibres [16]. For this reason, surface EMG is used in this study.

The captured MES appears Gaussian, and simply averaging the unprocessed signal provides no useful information about the signal. The average value of the unprocessed MES should be zero. Other data reduction procedures can be used to obtain useful information about the MES. These include rectifying, finding the mean or integrating the MES, but the Encyclopedia of Medical Devices and Instrumentation recommends using the root-mean-square (or RMS) value. The RMS value provides a measure of the energy of the signal [16].

The main disadvantages to using surface EMG are that it is only useful for detecting the MES from superficial muscles and that it cannot be used on smaller muscles. When using surface EMG on smaller muscles "cross-talk" can be detected from adjacent muscles [16]. 


\subsection{Stiffness}

In mechanical engineering, the term stiffness generally represents the relationship between force and resulting deformation of an elastic body. The stiffness of a spring refers to the relationship of the applied load and resulting extension of the spring; this relationship is represented by the spring constant. Stiffness also applies to concepts encountered in biomechanics. Muscle stiffness, joint stiffness and impedance are distinct yet related concepts, and they are discussed in the following three sections.

\subsubsection{Muscle Stiffness}

Muscle stiffness is a combination of the passive and active properties of the muscle.

The passive properties of the muscle are the properties of the muscle at rest, and they arise from the composition and structure of the muscle itself, specifically the amount and geometry of the connective tissue in the muscle. Connective tissues of the muscle control the resistance to stretching beyond resting length. A muscle stretched beyond its resting length generates a passive force that opposes lengthening.

The active properties of muscle arise from the ability to form cross-bridges. At resting length, where the length of the sarcomere is approximately $2.5 \mu \mathrm{m}$, the maximum number of cross-brides can form between filaments, and thus maximum tension is possible [17]. According to Winter [17], cross-bridges are able to form when the sarcomere measures between $1.5 \mu \mathrm{m}$ and $4.0 \mu \mathrm{m}$, which is approximately the equivalent of $60 \%$ to $160 \%$ of sarcomere resting length; in this range muscles are able to generate tension. Below $60 \%$ resting length, there is too much overlap of the contractile proteins to form cross-bridges necessary to generate a contraction; above $160 \%$ resting length there is no overlap of the contractile proteins making it impossible for cross-bridges to form and generate a contraction. 

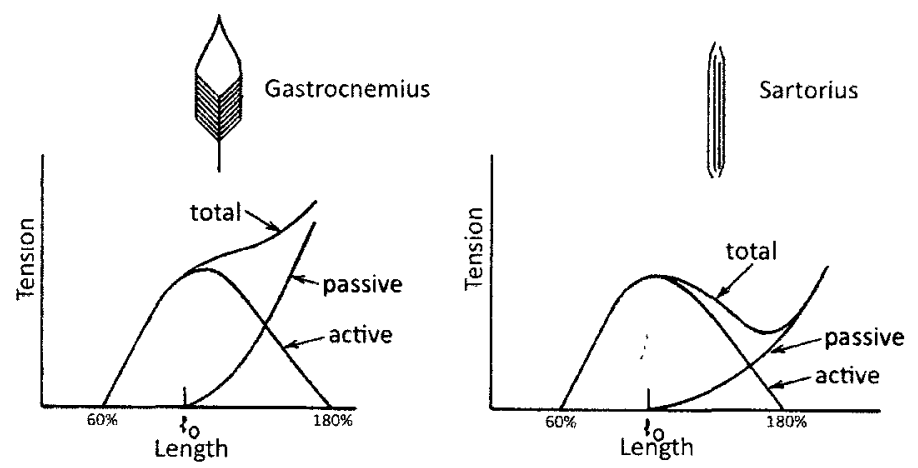

Figure 2.7: Schematic of force-length curves of a healthy gastrocnemius (left) and healthy sartorius (right). The total stiffness of a muscle, hence the force it can generate, is dependent on both its passive and active properties. Adapted from McMahon's Muscles, reflexes, and locomotion [18].

The relationship between the strength of an isometric contraction and the corresponding muscle length is shown in Figure 2.7. At an ideal length, which is typically close to resting length, a muscle is able to generate the maximum active force. The force a muscle is able to generate decreases as the muscle is shortened or stretched beyond this length. The shape of the force-length relationship is generally the same from muscle to muscle. Conversely, passive stiffness is not constant from muscle to muscle because this property depends on the amount and geometry of the connective tissues in the muscle. Consequently, the shapes of the total muscle tension curves vary from muscle to muscle, as demonstrated in Figure 2.7, which shows the total muscle stiffness curves for the gastrocnemius and sartorius. The gastrocnemius has short fibres and a relatively large amount of connective tissue. By contrast, the sartorius has long muscle fibres arranged in parallel. The total muscle stiffness curves of these two muscles differ: the total muscle stiffness curve of the sartorius has a local minimum and a more pronounced local maximum on its total muscle stiffness curve, as shown in Figure 2.7. 
The active stiffness of a muscle arises from reflex and voluntary contractions, and is regulated by changing muscle activation. Muscle activation can be quantified using EMG, which measures the electrical activity of muscles and is related to the level of muscular contraction. In their 1999 study of multijoint muscle regulation mechanisms, Osu and Gomi [19] quantified total muscle stiffness by measuring the individual muscle activity, multiplying the value of muscle activity by a positive constant coefficient, and adding a value corresponding to the intrinsic stiffness value of the inactive muscle (i.e. passive muscle stiffness). Their calculation assumes that muscle activity is proportional to EMG.

\subsubsection{Joint Stiffness}

Joint stiffness is a rotational stiffness, and it refers to the change in the applied moment relative to the change in joint rotation. Muscle stiffness (both its passive and active components) influences joint position and rotation, and thus contributes to joint stiffness. The passive properties of muscle are one of the determinants of equilibrium joint position. The elastic forces generated by muscles will return a hand to its original posture when it is displaced from its equilibrium position [20]. Active muscle stiffness (i.e. stiffness due to muscular contraction) allows joints to move, thus contributing to joint stiffness. It was observed by Osu and Gomi that there is a strong correlation between effective muscle stiffness and joint stiffness when muscles are working as agonists; this correlation is not as strong but can still often be found when muscles are working as antagonists [19].

There are multiple studies that have examined movements or held positions of the arm in the horizontal plane, thus keeping the influence of gravity constant [21], to study how these relate to joint stiffness (and limb impedance) [19-24]. While the human arm can be represented by a three-link model (upper arm, forearm and hand 
segments with joints at the shoulder, elbow and wrist), it is common for researchers to fix the wrist joint, by means of a splint or otherwise, so that rotation is only possible at the shoulder and elbow joints, allowing the arm to be represented by a two-link model.

In 1985, Mussa-Ivaldi et al. [22] developed a novel experimental method to characterize the spring-like behaviour of the neuromuscular system. They used a system where the wrist was constrained and fixed to the handle of a manipulandum (meaning the arm could be modelled as a two link system) and applied small displacements, of either 5 or $8 \mathrm{~mm}$ in magnitude, in various directions to the hand using five different hand positions in the horizontal plane. The movement that followed the applied disturbance lasted approximately $120 \mathrm{~ms}$. This was followed by a holding phase in the displaced position for $1.5 \mathrm{~s}$. Participants were told to focus on perceiving the direction of the displacement and to move rapidly in the direction opposite to the displacement so that the voluntary response could be observed. The researchers used EMG to measure activity of four muscles controlling the shoulder and elbow joints to determine the onset of the voluntary response, which occurred $300 \mathrm{~ms}$ to $800 \mathrm{~ms}$ into the holding phase, and to verify the absence of significant voluntary response during the first part of the holding phase. They calculated displacement and force vectors from measured joint angles and torque. During the holding phase, the researchers observed that the participant exerted a significant and measurable force on the handle. This force had no viscous or inertial components because the hand was at rest, thus the force represented the static restoring force propelling the hand back to its original equilibrium position. From their measurements of displacement and force, Mussa-Ivaldi et al. were able to determine stiffness, which they represented 
mathematically as a matrix, $\vec{K}$ shown in Equation 2.1,

$$
\begin{gathered}
\qquad \vec{F}=\vec{K} d \vec{x} \\
\text { where } \vec{K}=\left[\begin{array}{ll}
K_{x x} & K_{x y} \\
K_{y x} & K_{y y}
\end{array}\right]
\end{gathered}
$$

so that Equation 2.1 can be expressed as,

$$
\left[\begin{array}{l}
F_{x} \\
F_{y}
\end{array}\right]=\left[\begin{array}{ll}
K_{x x} & K_{x y} \\
K_{y x} & K_{y y}
\end{array}\right]\left[\begin{array}{l}
d x \\
d y
\end{array}\right]
$$

and graphically as an ellipse. The ellipse was defined by its size (area), shape (the ratio of the axes) and orientation (direction of the major axis). Arm posture was shown to produce regular patterns of change in the shape and orientation of the stiffness ellipse, as in Figure 2.8; however, no systematic changes in the magnitude of the stiffness ellipse could be characterized with changing arm posture. Additionally, in a given posture, the magnitude of the ellipse changed over time but shape and orientation did not [22].

In Osu and Gomi's 1999 study [19], they used a similar setup as Mussa-Ivaldi et al. to investigate multi-joint muscle regulation mechanisms by determining human arm stiffness from EMG measurements. Osu and Gomi's methodology differed from that of Mussa-Ivaldi et al.: Osu and Gomi required their participants to produce specified forces without co-contraction, to produce no external force with varying levels 

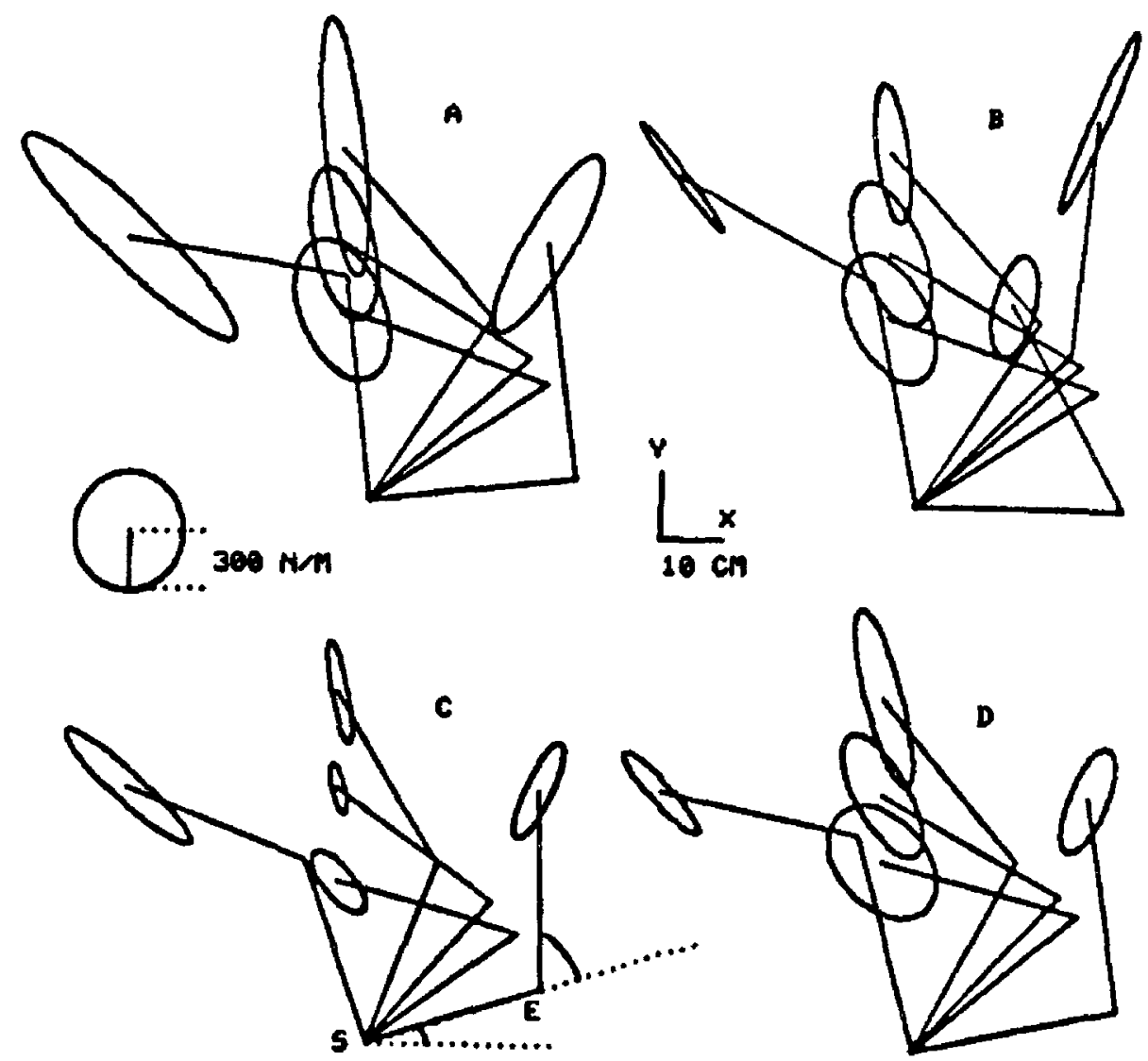

Figure 2.8: Estimated stiffness ellipses for different hand positions of subjects $\mathrm{A}, \mathrm{B}$, $\mathrm{C}$ and D in Mussa-Ivaldi et al.'s 1985 study [22]. The upper arm and forearm are represented by two line segments; the shoulder is located at S, and the elbow is located at E. Note that the shape and orientation of the ellipse vary with arm position. 
of co-contraction (none, quarter, half, full, co-contraction at the shoulder only, cocontraction at the elbow only), and to produce a specified force in specified directions with moderate co-contraction at five different positions in the horizontal plane. The study used a plastic cuff to fix the wrist joint of the participants, so that the arm could be modelled as a two-link manipulator. The cuff was coupled to the handle of the parallel link drive air-magnet floating manipulandum (PFM). The MES was captured from four monoarticular muscles and two biarticular muscles that correspond to idealized muscles in the two-link model. A monoarticular muscle crosses only one joint, and a biarticular muscle crosses two joints. The researchers assumed that EMG reflects the corresponding muscles stiffness, and used EMG measurement to calculate single-joint stiffnesses, which are the diagonal terms in the joint-stiffness matrix (or $K_{x x}$ and $K_{y y}$ in Equation 2.2), and cross-joint stiffnesses, which are the off-diagonal terms in the joint-stiffness matrix (or $K_{x y}$ and $K_{y x}$ in Equation 2.2), and hence, calculate joint stiffness. To match EMG levels to the stiffness measured by the perturbation method, they applied conversion factors; the limitation in doing so is that the conversion factors changed based on position and data groups [25]. In addition, this study, as well as another study by Osu et al. in 2002 [26], make the simplifying assumption that the moment arm remains constant.

Shin et al. [25] set out to improve upon Osu and Gomi's study, and developed a mathematical myokinetic (Mykin) model that takes into account anatomical and physiological data to estimate joint torque from EMG data. While they acknowledge that earlier studies, such as those by Mussa-Ivaldi et al. [22], Tsuji et al. [23] and Gomi and Kawato [24], were instrumental in developing the basic experimental approach to measuring stiffness, Shin et al. also refer to the perturbation method as being tedious, and point out that it relies on trial-to-trial repeatability [25]. They modelled the human arm as a two-link manipulator controlled by six monoarticular muscles and 
two biarticular muscles. In the first part of their experiments parameters representing muscle properties were determined from EMG and torque measurements. In the second part of the experiment, perturbations were applied to the participant's hand, while hand displacement and hand force were measured by the PFM during the perturbation period and the MES was collected using EMG equipment. The MES was used to calculate time-varying joint torque. These values were compared to the joint torques calculated from the force sensor and were found to be consistent. The Mykin model of Shin et al. successfully estimated joint torque using EMG measurements [25].

\section{Multi-joint Issues}

Because there can be multiple muscles that contribute to the stiffness of a joint, and those muscles can cross multiple joints, joint stiffness is also influenced by the proximity of other joints. This was somewhat alluded to in the previous section where the results of Osu and Gomi's 1999 study and Mussa-Ivaldi et al.'s 1985 study were described.

The endpoint stiffness matrix is a $2 \times 2$ matrix. The entries in the matrix represent single-joint $\left(K_{x x}\right.$ and $\left.K_{y y}\right)$ and cross-joint $\left(K_{x y}\right.$ and $\left.K_{y x}\right)$ stiffnesses. Monoarticular and biarticular muscles generate stiffness at a single-joint, whereas only biarticular muscles contribute to cross-joint stiffnesses [19]. By determining the joint-stiffness matrix, Osu and Gomi were able to show that the contribution of single-joint stiffnesses and cross-joint stiffnesses to the endpoint stiffness varies according to the nature of the task. For instance, Osu and Gomi observed that single-joint stiffness was always higher than cross-joint stiffness during static tasks, but that in some cases single-joint stiffness could be as small as cross-joint stiffness during dynamic tasks. This means that monoarticular muscles were not always activated during dynamic 
tasks, and that an increase in the biarticular muscle component of cross-joint stiffness was accompanied by an increase in the monoarticular muscle components of single-joint stiffness [19].

Researchers, such as Mussa-Ivaldi et al. [22], Flash and Mussa-Ivaldi [20], Tsuji et al. [23], Gomi and Kawato [24], Osu and Gomi [19] and Shin et al. [25], have observed a change in stiffness with changes in arm posture. This is manifested as a change in the shape and orientation of the stiffness ellipse. This further reinforces that joint stiffness is affected by the proximity to other joints.

\section{Impedance}

Impedance, like stiffness, is a measure of resistance to an applied force, but is not necessarily specific to a joint or muscle. Impedance can be used to characterize the overall response of the limb to a force while also taking into account the dynamic responses due to inertia and viscosity. When Tsuji et al. [23] published their work in 1995, previous studies had only estimated hand stiffness during multi-joint arm movements, not global impedance. Tsuji et al. used a similar approach to Mussa-Ivaldi et al.; they displaced the hand of a subject from an equilibrium position by applying a small disturbance for a short duration. It was essential that the disturbance be small so that hand inertia, viscosity and stiffness remain constant after the disturbance was applied. It was also essential that the duration be short to avoid any of the effects of voluntary neural feedback. A second-order linear model was used to estimate hand impedance based on time changes of the hand displacements and measured forces caused by the disturbances [23]. Like stiffness, hand inertia and viscosity can be represented mathematically by matrices and graphically by ellipses.

The researchers found that the major axes of the viscosity and stiffness ellipses 


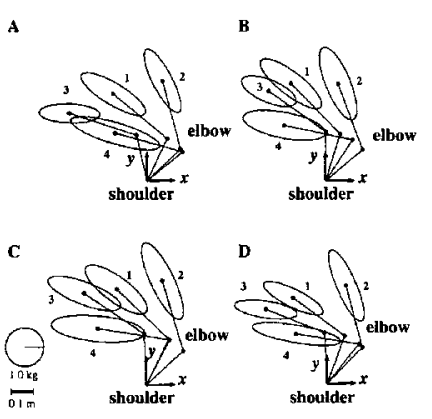

(a) Inertia ellipses

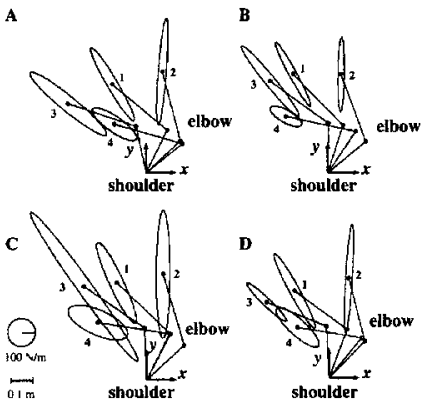

(b) Stiffness ellipses

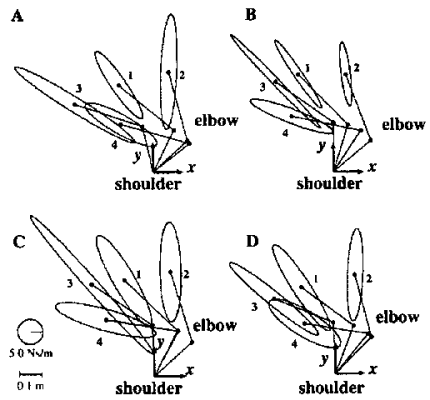

(c) Viscosity ellipses

Figure 2.9: Estimated inertia ellipses, stiffness ellipses and viscosity ellipses of several hand positions of all four participants (A, B, C and D) in Tsuji et al.'s 1995 study. Obtained from [23]. The upper arm and forearm are represented by two line segments, and the locations of the shoulder and elbow are labelled.

were nearly coaligned (see Figure 2.9). This was not unexpected because hand viscosity and stiffness are both dependent upon the viscoelastic properties of skeletal muscles, low-level neural reflexes and passive elements (e.g. skin and veins). The stiffness ellipses tended to be longer in distal locations of the workspace, became more isotropic in proximal locations of the workspace, and the major axes had a tendency to be oriented toward the participant's shoulder. Conversely, there was a tendency for the major axes of the inertia ellipses to be aligned with the forearm (see Figure 2.9). The results of Tsuji et al. were spatially comparable to the results of Mussa-Ivaldi et al.; however, the stiffness ellipses determined by Tsuji et al. were smaller in magnitude than those reported by Mussa-Ivaldi et al. [23].

\subsection{Co-contraction}

Studies employing the perturbation method to measure the endpoint stiffness using a manipulandum have been useful in characterizing the multi-joint behaviour of the arm in a defined workspace. It would be useful to extend these studies to situations 
where the arm does not need to be represented as a two-link model and where motions of the arm are not restricted to perturbations applied to the hand from different locations in the workspace, to understand better the joint stiffness in dexterous (and dynamic) tasks such as playing the piano. As stated in Chapter 1, many professional and non-professional piano players suffer from pain and injury, especially at the wrist. By better understanding the biomechanics of piano playing it might be possible to understand the causes of the pain and injury, and to develop corrective measures to treat these causes or minimize their effects. Measuring muscle activity and cocontraction using EMG provides a less restrictive alternative to the perturbation method.

Co-contraction typically occurs when two muscle groups with opposing actions surrounding a joint simultaneously contract [27]. The pair of antagonistic muscles produce forces, but in opposing directions with no net movement. Co-contraction serves as a motor control strategy to increase joint stability [28,29], improve movement accuracy [28] and adapt to changing environmental conditions [29]; however, these can occur at the cost of a reduction in mechanical efficiency [28]. When co-contraction becomes excessive, movement is impaired [30].

Measuring muscle activity and co-contraction provides an indication of changes in stiffness. Muscle activity as measured by EMG is an indication of the level of active stiffness of the muscle. Active muscle stiffness contributes to overall muscle stiffness, and muscle stiffness is a component of joint stiffness. An increase in co-contraction will be accompanied by an increase in joint stiffness; a decrease in co-contraction will be accompanied by a decrease in joint stiffness. 


\subsubsection{Quantifying Co-contraction}

Since measurement of co-contraction can be done using EMG, co-contraction can be assessed in monoarticular muscles, biarticular muscles and multiarticular muscles while performing movements in the normal physiological range, unlike the restrictions imposed by the studies described in Section 2.3.2. Even though co-contraction measurements offer a direct and computationally simpler method of observing changes in stiffness, there is no universally accepted method of calculating co-contraction. Co-contraction is usually expressed in one of two forms,

- As a ratio, or

- As the area of overlap of two linear envelopes (or traces) from two EMG signals, also called the co-contraction index (or CI),

or as a combination of these two forms.

\section{Co-contraction as a Ratio}

There are multiple types of ratios researchers use to express co-contraction. Researchers who have used a ratio of antagonist:agonist, or some other similar expression, to quantify simultaneous activation of an antagonistic pair of muscles include,

- Myklebust et al. [31],

- Levin and Hui-Chan [32],

- Ikeda et al. [33],

- Busse et al. [34],

- Damiano et al. [30], 
- Chae et al. [35].

Myklebust et al. [31] looked at the ratio of the activity of the tibialis anterior (or TA) to the activity of the gastrocnemius and soleus (or SOL) muscles. (The TA is on the ventral side of the lower leg, and the SOL muscles are on the dorsal side of the lower leg.) The researchers administered tendon taps to tendons on the ventral and dorsal sides of the lower leg, and quantified the simultaneous activation of the TA and SOL muscles by computing the ratio TA:SOL [31]. Even though part of the time the TA acted as the agonist and the SOL group acted as the antagonist (and vice versa), the ratio computed was always TA:SOL, not SOL:TA, so that the ratio was expressed consistently.

Levin and Hui-Chan's study published in 1994 [32] measured the activities of the tibialis anterior and soleus muscles during maximal isometric contractions. Cocontraction was calculated by computing the ratio of antagonist:total activity, where the total activity is the summation of agonist and antagonist activities.

There is also a lack of consensus on what co-contraction actually is. The definition of co-contraction used thus far (and used in the remainder of this document) is what Ikeda et al. refer to as co-activation [33]. In their study published in 1998, they maintain that co-activation is a measure of the electrical activity produced by muscles at a joint, and is defined as,

$$
\text { Co-activation }=\frac{\text { antagonist } \mathrm{EMG}}{\text { maximum } \mathrm{EMG}} * 100
$$

while they define co-contraction as the percentage of the net moment attributed to the antagonist moment, and they calculate co-contraction using the equation:

$$
\text { Co-contraction }=\frac{\text { antagonist moment }}{\text { net moment }} * 100
$$


Conversely, Busse et al. [34] used the following equations to define co-activation in their 2006 paper:

$$
\text { Hamstrings muscle moment }=\text { constant }(b) * \text { Hamstring EMG }
$$

Quadriceps muscle moment $=$ constant (a) * Quadriceps EMG

$$
\text { Co-activation }=\frac{b * \text { Hamstring EMG }}{a * \text { Quadriceps EMG }} * 100 \%
$$

Busse et al.'s study looked at co-activation during knee extension using an isometric exercise and a sit-to-stand task. Their computation of co-activation is a ratio of muscle moments, which is not dissimilar from Ikeda et al.'s definition of co-contraction. This further reinforces the inconsistencies in defining and calculating co-contraction as well as co-activiation.

Cowan et al. [36] used the Pearson Product-Moment (PPM) correlation to quantify muscular co-activation from EMG data. The PPM correlation coefficient is calculated according to the equation,

$$
\frac{\sum\left(x_{\imath}-x\right)\left(y_{\imath}-y\right)}{\left(\sum\left(x_{\imath}-x\right)^{2}\left(y_{\imath}-y\right)^{2}\right)^{1 / 2}}
$$

where $\mathrm{x}_{\imath}$ and $\mathrm{y}_{\imath}$ are magnitudes of the EMG linear envelopes of antagonistic muscles at time $\mathrm{i}$, and $\mathrm{x}$ and $\mathrm{y}$ are the average EMG levels of the antagonistic muscles. This method produces a ratio, thus it can be classified as another method of quantifying co-contraction.

In Damiano et al.'s study [30] of isometric strength and gait in children with cerebral palsy, published in 2000, co-contraction was quantified by determining the co-contraction ratio (or CCR). The co-contraction ratio was computed by comparing the EMG values of the agonist and antagonist for each point in time. Of the two 
values, the minimal EMG value was divided by the maximal value, so that the ratio was always $<1$. The CCR was computed by finding the mean ratio for the entire trial [30]. They found that the CCR was always expressed as antagonist:agonist in the isometric strength tests [30].

Chae et al.'s approach [35] was similar to that of Damiano et al. They used the ratio of the RMS values of the antagonist and agonist muscles to quantify cocontraction (i.e. RMS $\mathrm{Rntagonust}_{\text {: }} \mathrm{RMS}_{\text {agonıst }}$ ).

Yoshie et al. [37] quantified the co-contraction of the EDC and FDS using the relative difference signal (RDS), which is calculated for each point in time using the equation,

$$
R D S=\frac{(E-F)}{(E+F)}
$$

where E and F represent the rectified and smoothed EMG signals of the EDC and FDS, respectively, each scaled so that it has a mean of 1 . Unlike the previously described ratios used to quantify co-contraction, the RDS is not a single numerical value; rather, it is a series of values. The standard deviation of the RDS is calculated, which generates a single value called the reciprocal contraction index (or RCI). A higher RCI in an indication of a tendency towards reciprocal activity, and a lower $\mathrm{RCI}$ is an indication of a tendency towards co-contraction.

One major disadvantage to representing co-contraction as a ratio is that it does not indicate the magnitude of the contraction in the agonist and antagonist. A pair of antagonistic muscles with low levels of contraction could potentially yield the same CCR as a pair of antagonistic muscles with high levels of contraction. These two scenarios have very different impacts on muscle function. The latter could impair movement, whereas the former may stabilize the same movement. Moreover, high co-contraction may not indicate a high contribution by the antagonist muscle, but 
rather a low contribution by the agonist muscle [30].

This list is not exhaustive. Each of these studies has been described simply to show there is no consistency in the ratio used to quantify co-contraction, and that there are conflicting definitions of co-contraction.

\section{Co-contraction as the common overlap of two EMG signals}

Similar to the dilemma of defining co-contraction as a ratio, there is a lack of consensus on calculating co-contraction when it is defined as the common overlap between two EMG signals.

One common approach is to use the area of overlap of activities of the agonistantagonist muscle pair. Unnithan et al. defined this as the co-contraction index (or CI) [38]. In their assessment of co-contraction in muscles of the lower leg, they used $\mathrm{EMG}_{\text {vastus lateralss }} \cap \mathrm{EMG}_{\text {hamstrings }}$ to assess co-contraction in the thigh, and $\mathrm{EMG}_{\text {tzbralis anterior }} \cap \mathrm{EMG}_{\text {soleus }}$ to assess co-contraction in the lower leg, after raw EMG data were full wave rectified, low pass filtered with a cut-off frequency of 3 $\mathrm{Hz}$ and normalized [38]. The CI was found by dividing the area of overlap by the number of data points, and this is shown in Figure. 2.10. Similarly, Lamontagne et al. assessed the co-activation duration of the tibialis anterior (TA) and medial gastrocnemius (MG) by taking the overlap of the linear envelope of the TA and MG signals above a threshold voltage of $20 \mu \mathrm{V}$. Since they were analyzing gait, they also calculated the duration of co-activation by dividing the co-activation by the length of time over which co-activation was calculated. Co-activation duration was expressed as a percent [39].

Thoroughman and Shadmehr [40] also used the same technique to calculate cocontraction, which they call "wasted contraction," in their paper published in 1999. 


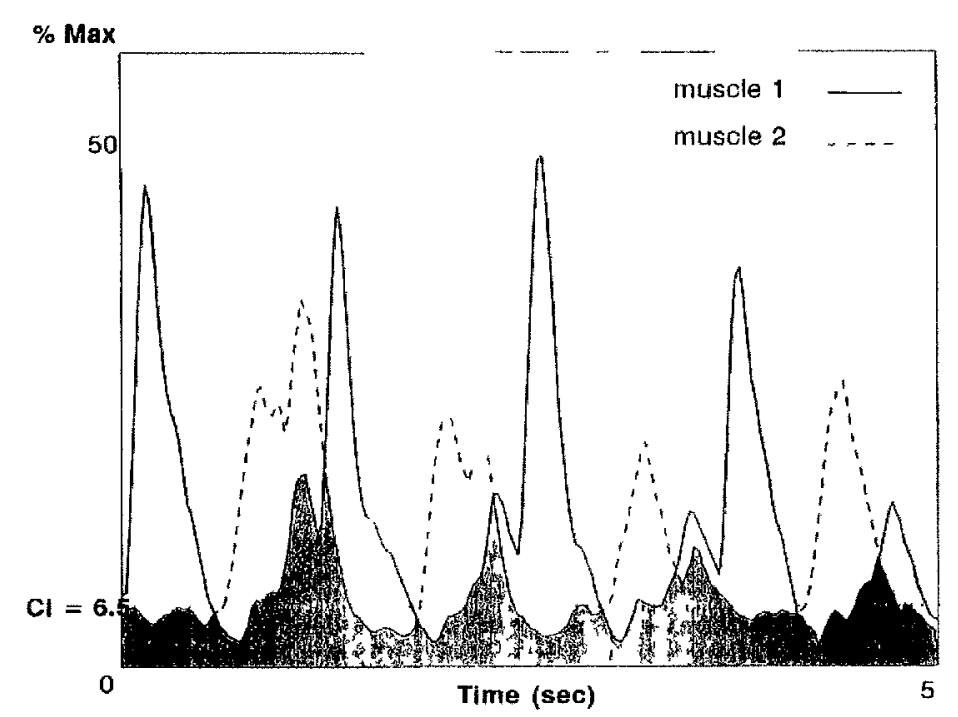

Figure 2.10: Co-contraction index of the vastus lateralis and hamstrings from Unnithan et al.'s study [38]. To find the CI, the area of overlap of linear envelopes of the vastus lateralis and hamstrings is divided by the number of data points.

They state that wasted contraction arises from contraction of opposing groups of muscles that does not lead to effective force production.

In Damiano et al.'s study [30] published in 2000, in addition to quantifying cocontraction using the CCR, they also evaluated the magnitude of co-contraction using the co-contraction magnitude (or CCM), which is another way of describing the area of overlap of the linear envelopes of the MES from a pair of antagonistic muscles.

\section{Co-contraction as a combination of a ratio and the area of overlap of two}

\section{EMG signals}

In his book titled Bromechanucs and Motor Control of Human Movement, Winter [17] employed a method of measuring the percentage co-contraction, which incorporated both a ratio and the area common to two EMG signals The percentage co-contraction is calculated as two times the area under the MES common to both 
the agonist and antagonist, divided by the sum of the areas under the MES of the agonist and antagonist, multiplied by $100 \%$ :

$$
\% \text { Co-contraction }=2 * \frac{\text { MES agonist } \cap \text { MES antagonist }}{\text { MES agonist }+ \text { MES antagonist }} * 100 \%
$$

This equation assumes that the activity of the antagonist is matched by an equal increase in the activity of the agonist, thus any activity that is unnecessary (or any "unnecessary cocontractions") must be equal to twice the activity of the antagonist alone, which is where the factor of 2 comes from in Equation 2.10 [17]. This method of calculating co-contraction does not require the signals to be normalized.

\section{Proposed calculation of co-contraction}

In this study, we propose using the geometric mean to calculate the co-contraction of an antagonistic muscle pair:

$$
\text { Co-contraction }=\left(M E S_{\text {agonist }} * M E S_{\text {antagonist }}\right)^{1 / 2}
$$

This calculation is computationally simple and offers an advantage over representing co-contraction as a ratio. According to our proposed definition, smaller agonist and antagonist muscle activities correspond to lower levels of co-contraction, and higher agonist and antagonist muscle activities correspond to higher levels of co-contraction. In this study, EMG values are normalized using isometric MVC contractions of the muscles involved. Co-contraction is calculated for the FCU and ECU; it is called CC1 because these muscles have their primary action at the wrist. Co-contraction is calculated for the FDS and EDC; it is called CC2 because these muscles have a secondary action at the wrist. 


\subsubsection{Studies Using Co-contraction}

Co-contraction is measured and studied during the performance of a variety of static and dynamic tasks. For instance, in Damiano et al.'s study [30], CCR and CCM were calculated for isometric strength tests (knee flexion and extension), which are static tasks, and during a walking trial, which is a dynamic task. Similarly, in Busse et al.'s study [34], co-contraction was quantified for an isometric knee extension exercise and also during a sit-to-stand task. Their study looked at co-contraction in healthy subjects and subjects with neurological disorders. They were able to show that co-contraction did not vary with the type of neurological pathology.

There is a wide variety of dynamic tasks during which co-contraction has been studied. Some of these recent studies are summarized in the next five paragraphs.

Caty et al. [41] studied the co-contraction of the FCU and ECU in swimmers during two principal phases of the freestyle stroke, insweep and outsweep, to investigate stabilization of the wrist. While the article did not use a direct measure of co-contraction, activation of an antagonist pair of muscles (the FCU and ECU) was quantified by integrating the rectified MES. They found that during the insweep and outsweep phases, FCU activation was similar, but that ECU activation was significantly higher during insweep than during outsweep. They state that the high resistance of the water on the hand during the insweep phase would have created an unstable load, and offer this as a possible explanation for increased ECU activity observed [41].

Heiden et al. [42] compared gait in patients with knee osteoarthritis to matched controls and looked at kinematic, kinetic and muscle co-contraction. Co-contraction ratios indicated that osteoarthritis patients had higher lateral muscle activation, which was matched by larger adduction moments. The researchers were able to 
conclude that the increase in lateral muscle activation observed in osteoarthritis patients was to stabilize the external knee adduction moment, which was related to lower perception of pain [42].

In a landing task, Yeadon et al. [43] were able to show there were high levels of co-activation at touch down. They had their participant jump from three different heights, and in all cases the participant exhibited high levels of co-activation. They developed a model to determine if landing tasks could be accomplished without coactivation. This was possible, but only at a maximum height of $1.05 \mathrm{~m}$ [43].

Liu and Ji measured the MES emanating from shoulder and upper limb muscles during a driver's steering manoeuvre. The researchers were able to confirm the existence of co-contraction during steering [44].

To the knowledge of the author, there has only been one study published that has examined changes in co-contraction while playing the piano. Yoshie et al. [37] had a set of highly trained pianists perform a piece of their choice in two settings: in rehearsal and in competition. They measured co-contraction, as indicated by the RCI, of the EDC and FDS as part of their study on music performance anxiety. The RCI was found to be lower during competition than during rehearsal, an indication of a tendency towards co-contraction.

\subsection{Previous Work by Vant}

The goal of Vant's study [3] was to detect changes in impedance at the wrist during piano playing using a driving point impedance model, where the driving point was at the wrist. In Vant's study force perturbations were applied to the right wrists of participants while they played a piece composed by Ann Southam. Displacements of the wrist were measured during perturbations as an indication of a change in 
impedance [3].

The piece, shown in Figure. 7.1, was composed specifically for Vant's study. The goal of the piece of music was to induce an increase in stiffness in the player's wrist after one minute of playing, even in an experienced pianist. The tempo is initially slow, meaning the number of notes played per second - or note rate - is low, but as the piece progresses the time signature changes and note rate increases. The piece also has a gradual crescendo, meaning the loudness of the piece increases as the piece progresses. The note pattern of the piece is repeated with subtle modifications to the pattern so that it is largely unpredictable, forcing the participant to read the music, rather than "play by ear" or from memory. The first four bars of the composition are repeated at the end of the piece so that the two sections can be compared [3]. The piece requires minimal lateral movement of the arm, as each finger always plays the same note. Vant hypothesized that an increase in note rate and loudness would lead to an increase in impedance.

Due to limitations of the equipment, Vant's analysis was limited to bars 1 to 8 (where the note rate ranges from one note to three notes per second played by the right hand) and bars 22 to 26 (where the note rate ranges from one note to two notes per second played by the right hand), but the piece is at its loudest in bars 17 to 20, and note rate is highest in bars 9 to 20 (where four notes per second are played by the right hand). Vant found no statistically significant change in displacement [3].

One of the goals of the present study is to confirm the results of Vants work, but also to analyze a section of the piece, specifically bars 17 to 20 , that Vant's methodology prevented her from analyzing. Rather than measuring changes in displacement, EMG will be used to capture the MES from extrinsic muscles in the forearm that influence wrist position: the FCU, ECU, FDS and EDC. The MES emitted from the FCU and ECU will be used to calculate CC1; similarly, the MES emitted from the 
FDS and EDC will be used to calculate CC2. Although $\mathrm{CC} 1$ and $\mathrm{CC} 2$ are derived from active muscle stiffnesses of the FCU, ECU, FDS and EDC, active muscle stiffness and co-contraction are independent. Both values of active muscle stiffness and co-contraction levels will be reported in this study. In addition, this study will use a larger sample size so that statistical analyses can be performed. 


\section{Chapter 3}

\section{Experimental Approach}

It was set out at the end of the last chapter that the methodology followed in Vant's study imposed limitations on the analysis that could be performed on the data collected. In line with Vant's recommendation to use a different approach, our study used EMG to detect changes in co-contraction as an indicator of changes in wrist stiffness.

The goals of this study were twofold: the first was to observe if a change in wrist stiffness occurred, as indicated by a change in co-contraction, while the participants played several exercises on the piano; the second was to measure co-contraction of participants' muscles while they played the piece composed by Ann Southam and to observe if these were similar to Vant's findings. Participants were required to play the piece by Ann Southam, after completing two other piano playing tasks.

This study was approved by the Carleton University Research Ethics Board.

\subsection{Participants}

Eleven healthy subjects were recruited for this study; however, the results of two subjects had to be omitted for reasons explained in Chapter 5 . The remaining nine 
participants were from 21 to 37 years old (one male, eight females, mean age $=$ 26.2 years, $\mathrm{SD}=5.1$ ). Of these nine participants, only one reported some pain while playing. The subject met the following inclusion criteria for the study if he or she was: 1) 18 years of age or older; and 2) had more than 15 years of piano playing experience or was studying piano performance at the undergraduate level. Written consent was obtained from each subject. Refer to Appendix B for the letter of information, consent form and questionnaire.

\subsection{Equipment}

The Bagnoli ${ }^{\top M}$ 8-ch Desktop EMG system (Delsys ${ }^{\circledR}$ Inc., Boston, MA) was used to collect electromyographic data (see Figure 3.1 for experimental setup). Surface EMG signals have frequencies as high as $500 \mathrm{~Hz}$ [45]; following the Nyquist theorem, EMG data were collected at $1000 \mathrm{~Hz}$. DE-2.1 Single Differential sensors (Delsys ${ }^{\circledR}$ Inc., Boston, MA), shown in Figure. 3.2, were used to detect the EMG signal. These sensors consist of:

- two $99.9 \%$ silver parallel bar electrodes each measuring $1 \mathrm{~mm}$ by $10 \mathrm{~mm}$, and spaced $10 \mathrm{~mm}$ apart and

- the electronic circuitry for differential amplification of the EMG signal.

The electrodes are active electrodes and they have a high input impedance (> $\left.10^{15} \Omega / / 0.2 p F\right)$. The gain of the differential amplifier is $10 \mathrm{~V} / \mathrm{V} \pm 10 \%$; it has a common mode rejection ratio of $92 \mathrm{~dB}$ and the overall noise is $1.2 \mu \mathrm{V}$ (RMS, R.T.I.) [46]. The myoelectric signal was amplified $1000 \mathrm{x}$ by the main amplifier and fed into the computer by a data acquisition (DAQ) device, NI USB-6211 (National Instruments 


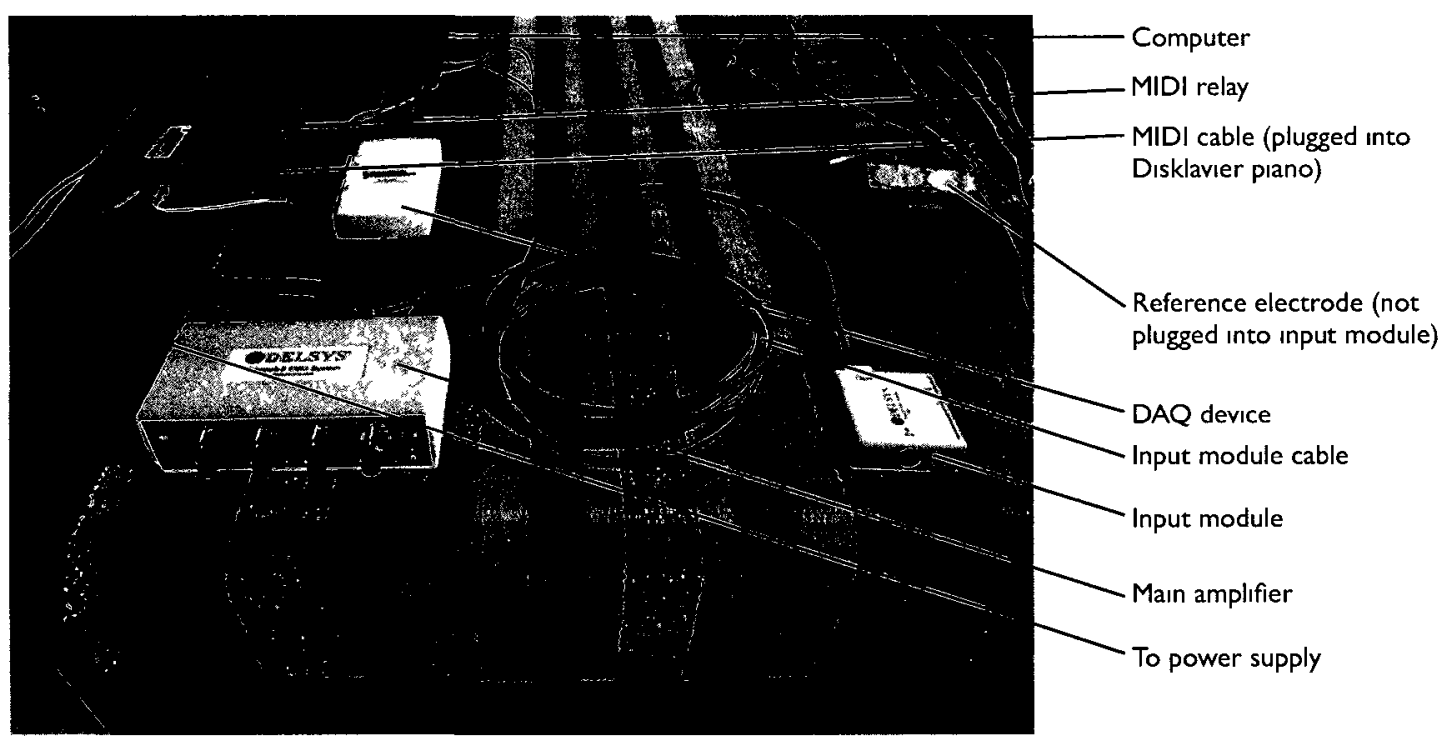

Figure 3.1: Bagnoli $^{T M}$ 8-ch desktop EMG system, DAQ device and MIDI relay.

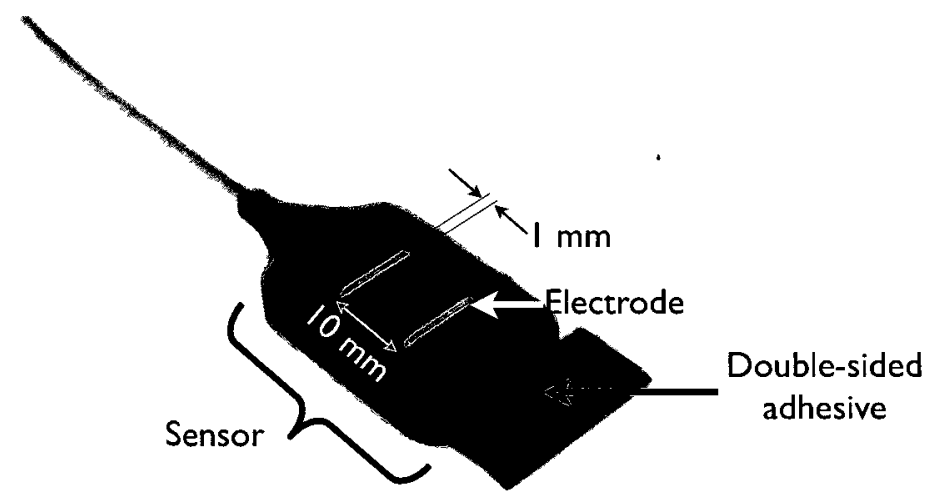

Figure 3.2: Delsys $^{\circledR}$ DE 2.1 single differential sensor with dimensions. 
Corporation, Austin, TX). The Bagnoli ${ }^{\top M}$ EMG system hardware uses a high pass filter (HPF) of $20 \mathrm{~Hz}$ and a low pass filter (LPF) of $450 \mathrm{~Hz}$. EMGworks ${ }^{\circledR} 3.7$ software (Delsys ${ }^{\circledR}$ Inc., Boston, MA) was used to collect the data.

A Yamaha C7 7'6" grand piano equipped with a Disklavier Pro Mark III optical sensor system (Yamaha Canada Music Ltd., Toronto, ON) was used to collect musical instrument digital interface (MIDI) data. This includes key velocity, note played and timing (note on and length of note) information.

EMG data collection did not begin at the same time as MIDI data collection. In order to align the two sets of data, a MIDI relay (MIDI Solutions Inc., Vancouver, BC) was employed. The MIDI relay was connected to the Disklavier piano and to the DAQ device (refer to Figure 3.3(a) for a schematic of the setup). The relay was programmed to respond to a MIDI event: G4 (or MIDI note number 67) on the Disklavier piano. When G4 was depressed, the relay closed, producing a change in voltage, which was measured by the DAQ device. The relay opened in response to G4 note-off and the voltage returned to approximately $0 \mathrm{~V}$. This is depicted in Figure $3.3(\mathrm{~b})$.

\subsection{Setup}

The quality of the myoelectric signal is influenced by the skin-electrode interface. When applying the electrodes to the skin, excessive amounts of body hair should be shaved or pushed aside so they do not interfere with the signal collected by the electrode. All of the participants in this study had very little amounts of hair on their forearms, and so shaving was unnecessary. The sites on the subject's forearm where the sensors were to be placed were cleaned with rubbing alcohol (95\% ethanol). The electrode portions of the Delsys ${ }^{\circledR}$ parallel bar EMG sensors were cleaned with rubbing 


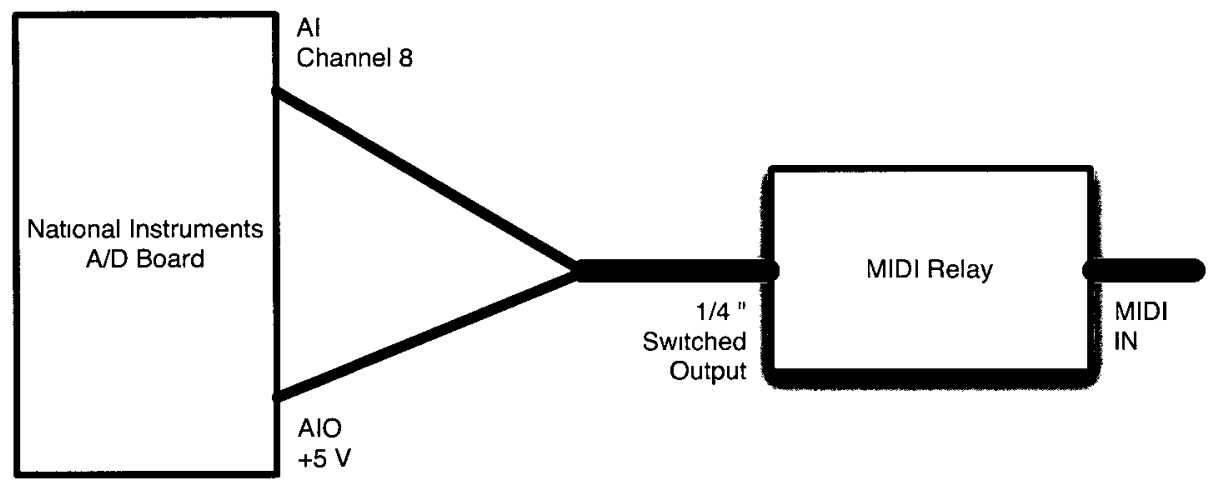

(a) MIDI relay connected to DAQ device and Disklavier

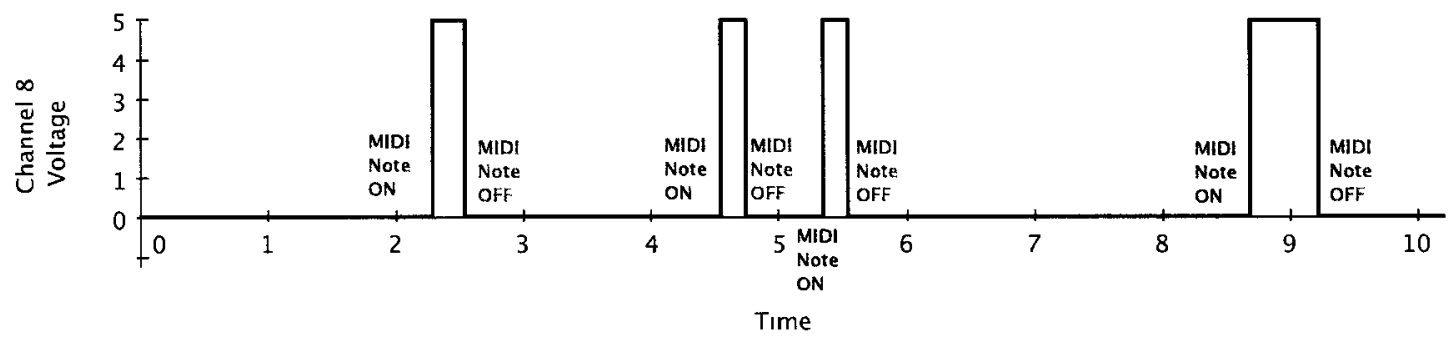

(b) Voltage change

Figure 3.3: (a) Configuration of MIDI relay, showing connections to DAQ device (to channel 8 and $5 \mathrm{~V} \mathrm{AIO} \mathrm{channel)} \mathrm{and} \mathrm{Disklavier} \mathrm{piano} \mathrm{(via} \mathrm{MIDI} \mathrm{IN} \mathrm{port).}$ (b) Resulting voltage change during G4 note on, note off. 
alcohol to remove any residue. No electrolyte was applied to the skin. Although electrolytes are commonly used to improved the electrical conductivity between the electrode and the skin, the electrodes used in this study are "dry sensors," meaning they do not require an electrolyte to make proper electrical contact with the skin [47].

Another factor to consider is sensor location. Sensor location influences the quality of the myoelectric signal obtained. Placing a sensor near a tendon origin, the innervation zone or the perimeter of the muscle will yield signals with lower amplitudes. The sensors were placed along the longitudinal midline of the muscle, with the silver bars perpendicular to the direction of the muscle fibres. Sensor placement is the most important factor in achieving a high signal-to-noise ratio and reducing crosstalk. Double-sided adhesive strips were used to fix the sensors to the skin overlying the bellies of

- the flexor carpi ulnaris (FCU),

- the extensor carpis ulnaris (ECU),

- the flexor digitorum superficialis (FDS), and

- the extensor digitorum communis (EDC)

of only the right arm. The sensors were placed according to the instructions in the Anatomıc Guıde for the Electromyographer [48] (see Figure. 3.4 for sensor placement). The ECU and EDC are muscles on the posterior side of the forearm; the FCU and the FDS are muscles on the volar side of the forearm. A reference electrode was placed on an area that is electrically unrelated: the lateral epicondyle of subject's right elbow. 


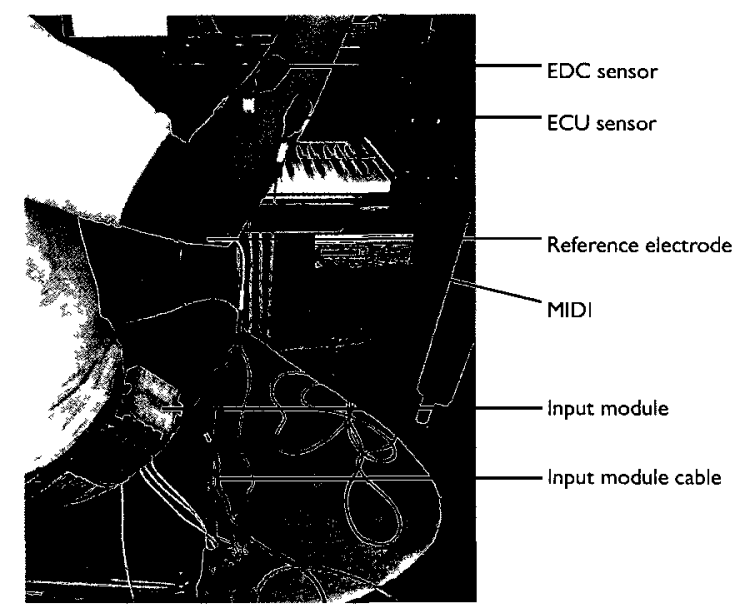

(a) Reference electrode, EDC and ECU sensors

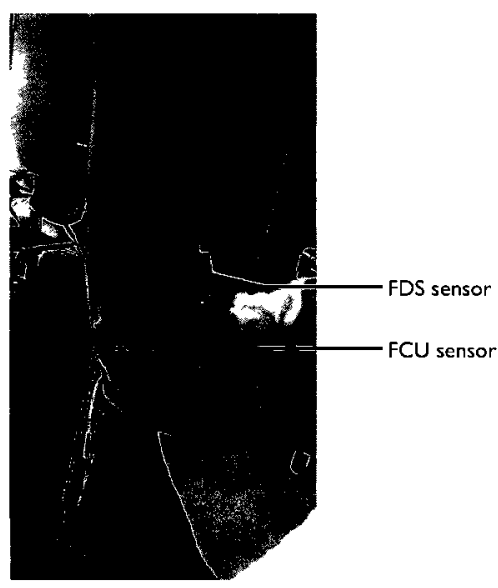

(b) FDS and FCU sensors

Figure 3.4: (a) Placement of EDC and ECU sensors. Location of the reference electrode is shown. (b) Placement of FDS and FCU sensors.

\subsection{Task}

Participants did not perform any warmup exercises before beginning the experiment. The participant entered the room, read the letter of information, and signed the consent form. The sensors were then affixed to the forearm of the participant as described in the previous section.

Before completing the three experimental tasks, the participant performed four maximal voluntary contractions (MVC), designed by the researchers. An MVC is an indication of a subject's strength and estimates the physiologic limits of the muscle [49]. When EMG results are expressed as a percentage of the MVC, they are said to be normalized. The purpose of normalizing the measurements is so that comparisons can be made between muscles belonging to the same person and between subjects. In the first MVC exercise, the participant flexed their hand towards their forearm while keeping a $90^{\circ}$ angle between the forearm and upper arm. In the second MVC exercise, the participant clenched his or her fist as hard as possible. The participant was asked to maintain a $90^{\circ}$ angle between his or her forearm and upper arm. In the 
third MVC exercise, the subject pulled on the back of a chair with the middle and distal phalanges; the PIP joint was flexed and the MCP joint was extended. In the fourth MVC exercise the subject placed the middle and distal phalanges underneath the top of a table and extended their fingers towards the ceiling. MVC exercises were done using only the right arm. Each contraction was held for approximately three seconds and participants were allowed to relax for one minute between contractions to minimize fatigue effects.

Muscles typically generate their maximum tension at an optimal resting length; however, during muscle shortening, an increase in EMG may occur to compensate for this shortening that is not attributable to increased force production. High levels of EMG activity are commonly seen at the end ranges of motion [50]. The experimental tasks chosen for this study use movements not necessarily controlled by isometric, isotonic contractions. For this reason, several MVC exercises were designed that utilize a variety of muscle positions. The highest overall calculated MVC value for each muscle across all tasks was used to normalize the results of all three playing tasks.

For the first experimental task, the participant played a four-octave C major scale, hands together, in sixteenth notes (where the quarter note $=108$ beats per minute ${ }^{1}$ ), ascending and descending, at a moderate loudness level (approximately mezzo piano), three times continuously.

The second experimental task was adapted from exercises by Ernó Dohnányi [51]. The participant played and held the root position of the D minor triad (MIDI note numbers 62, 65 and 69) with the right hand while alternately depressing the E4 key (MIDI note number 64) and G4 key (MIDI note number 67) with the second and fourth fingers, respectively, of the right hand 30 times as fast as he or she could. The

\footnotetext{
${ }^{1}$ This tempo was established using a metronome. Additional discussion about the meaning of tempo can be found in Section 5.1.1
} 
participant was then allowed to relax for one minute. The participant then played and held the first inversion of the A minor triad (MIDI note numbers 60, 64 and 69) with the right hand while alternately depressing the F4 key (MIDI note number 65) and the G4 key (MIDI note number 67) with the third and fourth fingers of the right hand, 30 times, as fast as he or she could. The musical score for these exercises is shown in Figure. 6.1, Chapter 6. It should be noted that participants did not play from the musical score; the task was demonstrated to the participants by the researcher, who held the alternating notes when demonstrating the task. An explanation of holding is given in Section 6.1.1.

For the third experimental task, the participant was asked to play a piece written by Ann Southam twice (see Figure 7.1, Chapter 7). This piece was composed to induce changes in wrist stiffness [3].

Separate EMG files and separate MIDI files were created for each experimental task (for a total of four MVC EMG files, five EMG [task] files and five MIDI files per participant). In addition to collecting EMG and MIDI data, videos of each experimental task were recorded.

Finally, the participants filled out a questionnaire regarding their playing habits before the end of the session. 


\section{Chapter 4}

\section{Data Processing and Preparation}

\subsection{EMG processing in EMGworks ${ }^{\circledR}$}

Raw EMG files were processed using EMGworks ${ }^{\circledR} 3.7$ software and MATLAB ${ }^{\circledR}$ 7.1 (The Mathworks ${ }^{\text {TM }}$, Inc., Natick, MA).

EMG files, including MVC EMG files, were imported into the EMGworks ${ }^{\circledR}$ analysis environment. All raw EMG data were baseline corrected by removing the mean, and software filtered using a fourth order Butterworth filter with a bandpass frequency of $20 \mathrm{~Hz}$ to $450 \mathrm{~Hz}$. At this stage, a three-second segment of data of the MVC EMG files where levels of contraction were highest was selected. The root mean square (RMS) was calculated for each data set (experimental task files and MVC files) using a moving window of $0.125 \mathrm{~s}$ and a window overlap of $0.0625 \mathrm{~s}$. Calculating the RMS with the window size and window overlap specified here reduced the number of data points by a factor of 63 . 


\subsection{Analysis of EMG and MIDI files in the MATLAB ${ }^{\circledR}$ Environment}

Following the processing and filtering of raw EMG files in the EMGworks ${ }^{\circledR}$ analysis environment, the resulting files were converted to .csv format so that they could be imported into MATLAB ${ }^{\circledR}$. A summary of the algorithms applied to the EMG and MIDI files recorded during the third task is shown in Figure 4.1. The parallelograms represent the algorithms created and used to analyze the data.

\subsubsection{Manipulation of EMG and MIDI files in MATLAB ${ }^{\circledR}$}

Files in .csv format were imported into MATLAB ${ }^{\circledR}$ using the LoadData algorithm. The code for this algorithm is given in Appendix C. Three functions were created for the purpose of analyzing EMG data collected in this study: $M V C$, NormalizeEMG and cocontraction. The code for these functions is given in Appendix D. The MVC function calculates the average value of each MVC exercise for each muscle, and finds the maximum value for each muscle (refer to the following section for more details on the calculation of the MVC); the NormalizeEMG function normalizes the EMG data, so that EMG data is expressed as a percentage of the MVC; the cocontraction function calculates co-contraction using the geometric mean. The LoadData algorithm contains instructions that use these three functions. Additionally, the LoadData algorithm was used to import raw (unprocessed) EMG files and MIDI files into MATLAB ${ }^{\circledR}$. 


\section{MVC EMG files}

For each subject, there were four MVC values calculated for each of the four muscles measured because four MVC exercises were used. The results of the three experimental tasks were normalized using the highest MVC value observed for each muscle, regardless in which of the MVC exercises it occurred; the EMG results of the three experimental tasks are expressed as a percentage of the MVC.

\section{MIDI files}

The MIDI Toolbox: MATLAB Tools for Music Research [52] was used to import MIDI files into MATLAB ${ }^{\circledR}$. These files were imported as structure arrays. Column 1 is note onset time in beats; column 2 is note duration in beats; column 3 is MIDI channel (which is always channel 1 in these sets of experiments); column 4 is MIDI pitch (also known as MIDI note number); column 5 is key velocity, which is a value between 0 and 127, and indicates how loudly the note is played; column 6 is note onset in seconds; and column 7 is note duration in seconds. Once MIDI files were imported into MATLAB, the files were adjusted by subtracting the initial start time (row 1, column 6) from all column 6 values. This was done so that the first note played occurred at $t=0$. A note off column was created (in column 8) by adding column 6 to column 7 .

\section{Ann Southam piece - MIDI files}

In the Ann Southam piece, only the notes played by the right hand were of interest because EMG data were collected from right forearm muscles. To remove the notes played by the left hand, an algorithm was created in MATLAB that eliminated notes with MIDI note numbers lower than 60. In the Ann Southam piece, the right hand does not play notes below MIDI note number 65 , and the left hand does not play 
notes above MIDI note number 55. The MATLAB code used to do this can be found in Appendix E.

\subsubsection{Synchronizing MIDI and EMG data}

As described in Section 4.2.1 MIDI files were adjusted so that $t=0$ corresponded to the first MIDI event (i.e. note on). EMG data collection began before the participant started playing the piano, so the first few seconds of EMG data needed to be trimmed from the file. This was accomplished by having a voltage spike fed into the EMG equipment in response to a particular MIDI event on the piano (i.e. G4 note on), and then shifting the EMG data so that the voltage spike aligned with the corresponding MIDI event. To do so, a MIDI relay was employed. The MIDI relay was connected to the Disklavier piano and the DAQ device. The relay was programmed by sending it a system exclusive (SysEx) message. HexEdit [53] was used to create a SysEx message, i.e. a message in .syx format, and SysEx Librarian [54] was used to send the SysEx message to the relay. The message instructed the relay to close when G4 was depressed, and this generated a voltage change that was detected by the DAQ device. When the G4 key was released, the voltage returned to approximately $0 \mathrm{~V}$. The first voltage change in the unprocessed EMG data was aligned with the first G4 from the MIDI data. This was done using the TrimData algorithm. The TrimData algorithm code for the first experimental task is found in Appendix F. The TrimData algorithm generates a value, $\mathrm{T}$. The value of $\mathrm{T}$ corresponds to the time delay between the start of the EMG and MIDI data (refer to Figure. 4.2). When $\mathrm{T}^{*} 1000$ number of rows are removed from raw EMG files and the value of $\mathrm{T}$ is subtracted from the time data (column 1 in all EMG files), raw EMG files align with MIDI data. When $(1000 / 63)^{*} \mathrm{~T}$ number of rows are removed from processed EMG files and the value of 


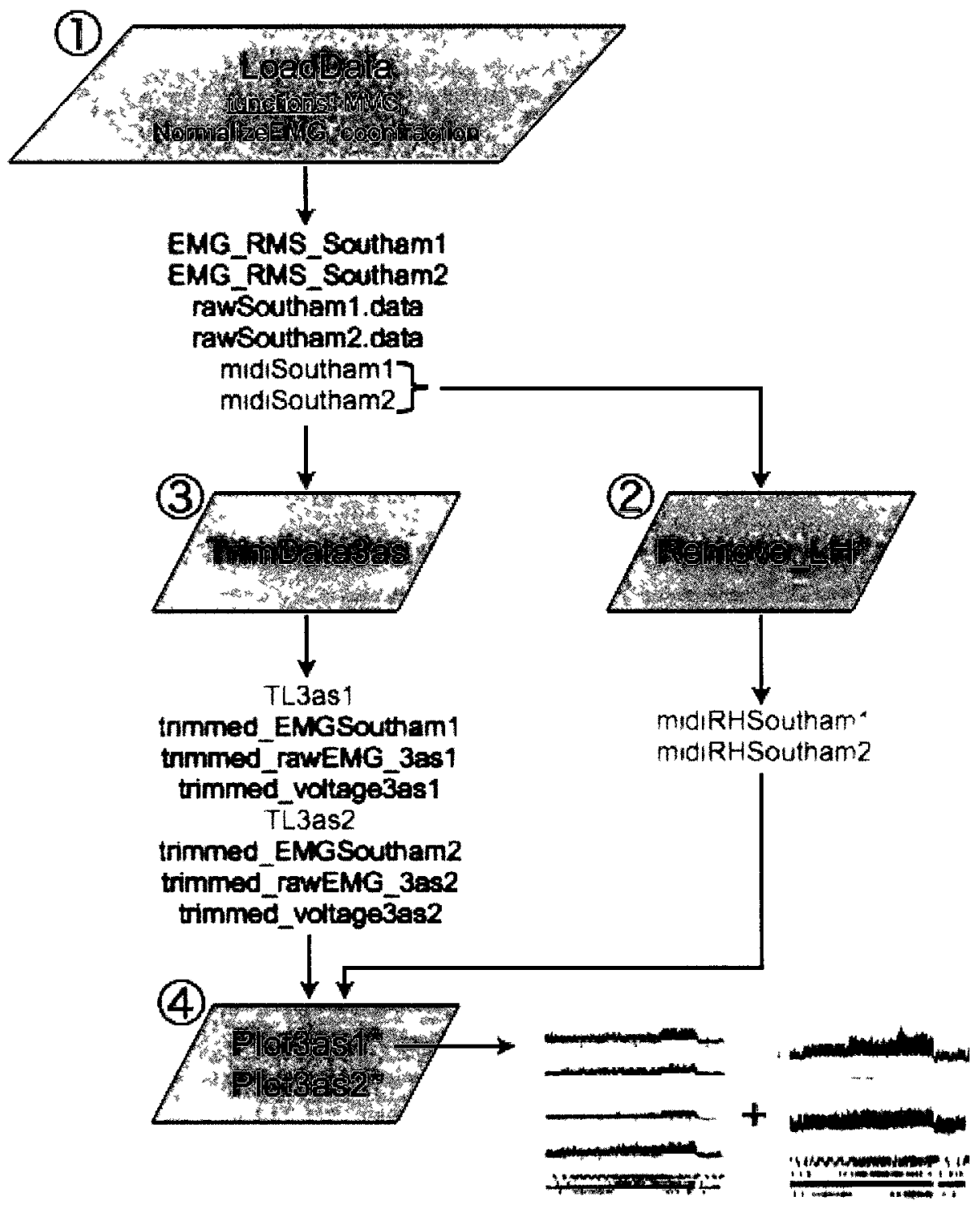

Figure 4.1: Flowchart showing the algorithms used to analyze the EMG and MIDI data of the Ann Southam piece. This series of algorithms was used when plotting the normalized EMG results and MIDI data. The algorithms are denoted by parallelograms. The variables output by the algorithms are represented by text. When the text is grey, that varıable represents MIDI data; when the text is blue, that variable originates from raw EMG data, when the text is green, that variable originates from processed EMG data. The * in the algorithm name indicates that some parameters are participant-specific and need to be changed each time the algorithm is run for a different participant. 
$\mathrm{T}$ is subtracted from the time data, processed EMG files align with MIDI data.

\subsubsection{Identifying sections in $\mathrm{C}$ major scale}

In the $\mathrm{C}$ major scale, the notes played by the right hand ranged from MIDI note number 48 to MIDI note number 96 . The ascending segment of the scale began at the time MIDI note number 48 was played by the right hand; the descending segment of the scale began at the time MIDI note number 96 was played by the right hand. The notes C3 (MIDI note number 48) and C7 (MIDI note number 96) were identified using the DetectSpike algorithm (see Appendix G for the code). The times associated with these notes were used to identify manually the time at which the directionality of the scale changed in the EMG files. The result was that the EMG files collected during the playing of the $\mathrm{C}$ major scale were split into six sections: three ascending segments (A1, A2, A3) and three descending segments (D1, D2, D3). The ascending segment terminated with the start of the next descending segment, and the descending segment terminated with the start of the next ascending segment (or when the last note was played, as was the case of the third descending segment). If there were a pause before playing the top note of the scale (MIDI note number 96), those EMG data were included in the ascending segment before MIDI note number 96 . In the rare case that the participant had a false start, the EMG data corresponding to those notes were cut out.

\subsubsection{Identifying sections in Ann Southam piece}

For reasons further explained in Chapter 7, the Ann Southam piece was split into 11 distinct sections: A, B, C, D, E, F, G, W, A', B' and W'. Musically speaking, section $\mathrm{G}$ could be considered part of $\mathrm{W}$, and section $W^{\prime}$ could be considered part 


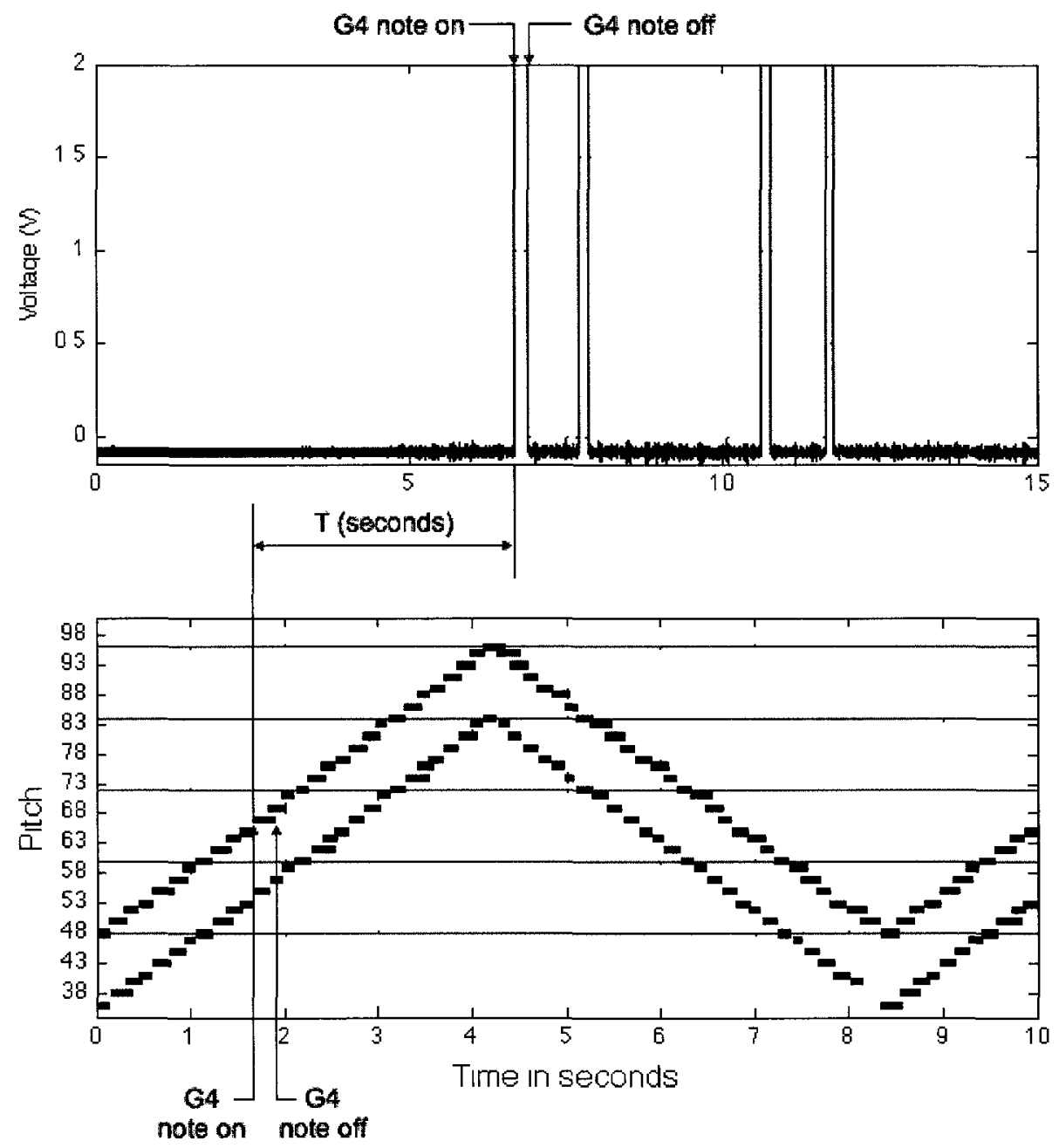

Figure 4.2: Unshifted voltage data as detected by channel 8 of the DAQ device and the resulting voltage change due to the depression of G4, corresponding MIDI data (bottom figure) and visual representation of $T$ - the time delay between the start of the EMG and MIDI date - in seconds as calculated by the TrimData algorithm. In this case, the first voltage change occurs at $\mathrm{t}=6.668 \mathrm{~s}$, and the first G4 in the MIDI data is at $t=1.671 \mathrm{~s}$, and the corresponding value of $\mathrm{T}$ is $4.997 \mathrm{~s}$. 
of $\mathrm{B}^{\prime}$ as both sections $\mathrm{W}$ and $\mathrm{W}^{\prime}$ are each composed of only one note. They were analyzed separately because over the course of $\mathrm{W}$ and $\mathrm{W}^{\prime}$, active muscle stiffness and co-contraction were observed to decrease. These divisions were based on note rate, note sequence and location in the piece. Where a section was repeated at the end of the piece, it was given the same letter as the first time it was played followed by a prime (').

Sections D, E, F and G all have the same note rate, but have different sequences of notes and different numbers of notes. Only section $\mathrm{G}$ will be compared to the other sections of the piece (A, B, C, W, A', B' and $\left.W^{\prime}\right)$ because it is expected that the greatest change in active muscle stiffness and co-contraction will have occurred by the time section $\mathrm{G}$ is played.

When playing the piece, many participants did not complete all repetitions, or they repeated sections $\mathrm{C}$ or $\mathrm{G}$ more times than indicated in the musical score (refer to Table 7.3 of Chapter 7). Additionally, the number of note accuracy errors in sections $\mathrm{C}$ and $\mathrm{G}$ varied widely (refer to Tables 7.1 and 7.2 for the number of accuracy errors made by participants in their first and second attempts, respectively, of the Ann Southam piece). Where participants made fewer or no errors in sections $\mathrm{C}$ and/or $\mathrm{G}$, the beginning and end of these sections were easily identifiable. This was not the case where participants made many errors - note accuracy and repetition/omission errors. To maintain some consistency in the lengths of sections $\mathrm{C}$ and $\mathrm{G}$, section $\mathrm{C}$ was defined as the 96 notes following section B; section G was defined as the 96 notes preceding section W. These redefined sections were called C96 and G96 respectively, so that they are not confused with the actual sections $\mathrm{C}$ and $\mathrm{G}$, which may be longer or shorter than 96 notes. Where sections $\mathrm{C}$ or $\mathrm{G}$ contained omission errors, they were named according to the actual number of notes they contained. For instance, in E010's first attempt of the Ann Southam piece, section C was 73 notes long, thus it 
was named $\mathrm{C} 73$.

The divisions were made manually: the note number column of the MIDI file (column 4) was used to identify the start and end of the sections of the Ann Southam piece. There are times associated with these start and end points, which were used to identify the times in the EMG data at which a section started and ended. 


\section{Chapter 5}

\section{Task \#1: Results and Discussion}

A total of eleven subjects underwent testing according to the protocol described in Chapter 3. On the basis of their responses to the questionnaire, all eleven subjects met the inclusion criteria of the study. Subjects were assigned numbers (E001 E011) to maintain anonymity. Subject E001 was very familiar with the experimental tasks, having participated in several pilot studies. In 2003, it was observed by Gribble et al. that over the course of practice of pointing movements, co-contraction and associated limb stiffness decreased [27]. Thus it is difficult to say which aspects of E001's results could be attributed to the nature of the exercises and which results could be attributed to the effect of practicing. There were problems with the EMG equipment when E011 underwent testing, and the data obtained were contaminated. For these reasons, the results of E001 and E011 are not included in this analysis.

\subsection{Results of Task \#1: C Major Scale}

Generally, the first scale learned when playing the piano is the $\mathrm{C}$ major scale because it uses only the white keys on the keyboard. Participants in this study were required 
to play the $\mathrm{C}$ major scale in parallel motion, ascending and descending, three times continuously, in sixteenth notes at $108 \mathrm{bpm}$, which is the equivalent of playing 7.2 notes per second per hand ${ }^{1}$ and corresponds to a late intermediate level. The tempo was established using a metronome, but participants were given the option of playing the scale with or without the metronome.

\subsubsection{Execution of C Major Scale}

Although no one played at exactly $108 \mathrm{bpm}$, most participants played close to that speed. Table 5.1 shows participants' mean ${ }^{2}$ note rates of the full scale, pooled ascending segments (A), each ascending segment (A1, A2, A3), pooled descending segments (D) and each descending segment (D1, D2, D3). In this study, the correct mean note rate of the $\mathrm{C}$ major scale was 7.2 notes/s. Where a participant's mean note rate was less than 7.2 notes/s, he or she played slower than the indicated tempo, and where a participant's mean note rate was greater than 7.2 notes/s, he or she played faster than the indicated tempo.

According to Table 5.1, participants E002, E004 and E009 played slower than the indicated tempo, participants E005 and E007 player faster, and participants E003, E006 and E010 played approximately at the indicated tempo. Not surprisingly, only participants E003 and E010 chose to play with a metronome. Participant E004 played slightly slower than all participants, and participant E009 played much slower than all participants.

The results in Table 5.1 also show that direction had an effect on the tempo. Some participants, including E002, E003, E004, E005 and E010, in most cases, played faster

\footnotetext{
${ }^{1}$ In the realm of music, the speed at which an exercise or piece is played is called the tempo and it is expressed in bpm, not notes per second.

${ }^{2}$ In this context, and in all other cases unless directly stated, the mean refers to the arithmetic mean.
} 
Table 5.1: Participants' mean note rates of the full scale, pooled ascending segments (A), each ascending segment (A1, A2, A3), pooled descending segments (D), and each descending segment (D1, D2, D3) of the C major scale, expressed in notes per second. When the scale is played at $108 \mathrm{bpm}$ the mean note rate is the same as it is in the correct case. Where a participant's mean note rate was less than 7.2 notes/s, he or she played slower than the indicated tempo, and where a participant's mean note rate was greater than 7.2 notes/s, he or she played faster than the indicated tempo. There was a problem with collecting MIDI data when participant E008 was tested, thus no MIDI data were collected and the mean note rate could not be accurately calculated. This is indicated by $a t$.

\begin{tabular}{|c|c|c|c|c|c|c|c|c|c|c|}
\hline & \multicolumn{9}{|c|}{ Note Rate (notes/s) } \\
\hline & & Full scale & A & A1 & A2 & A3 & D & D1 & D2 & D3 \\
\hline \multicolumn{2}{|c|}{ correct } & 7.20 & 7.20 & 7.20 & 7.20 & 7.20 & 7.20 & 7.20 & 7.20 & 7.20 \\
\hline \multirow{9}{*}{ 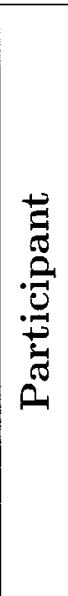 } & E002 & 6.67 & 6.74 & 6.78 & 6.67 & 6.78 & 6.60 & 6.68 & 6.64 & 6.48 \\
\hline & E003 & 7.20 & 7.25 & 7.24 & 7.25 & 7.27 & 7.14 & 7.16 & 7.12 & 7.14 \\
\hline & E004 & 6.10 & 6.10 & 6.18 & 6.11 & 6.02 & 6.09 & 6.28 & 6.02 & 5.98 \\
\hline & E005 & 7.63 & 7.65 & 7.71 & 7.65 & 7.59 & 7.62 & 7.67 & 7.69 & 7.49 \\
\hline & E006 & 7.15 & 7.20 & 7.24 & 7.05 & 7.31 & 7.11 & 7.06 & 7.16 & 7.11 \\
\hline & E007 & 7.87 & 7.85 & 7.84 & 7.80 & 7.91 & 7.88 & 8.07 & 7.91 & 7.67 \\
\hline & E008 & $\dagger$ & $\dagger$ & $\dagger$ & $\dagger$ & $\dagger$ & $\dagger$ & $\dagger$ & $\dagger$ & $\dagger$ \\
\hline & E009 & 5.07 & 5.10 & 4.90 & 5.14 & 5.26 & 5.05 & 4.79 & 5.11 & 5.27 \\
\hline & E010 & 7.18 & 7.29 & 7.31 & 7.27 & 7.27 & 7.08 & 7.04 & 7.11 & 7.11 \\
\hline
\end{tabular}


during the ascending segment than during the descending segment. The remaining three participants showed no common pattern in terms of tempo. Participant E006 played the first ascending and descending segments at approximately the same tempo, but played the remaining two ascending segments much slower than the remaining two descending segments. Participant E007 played with very little variability in tempo. Participant E009 played consistently faster with each repeat of the scale.

\subsubsection{Hypotheses — C Major Scale}

The $\mathrm{C}$ major scale is a rudimentary part of piano technique. A classically trained pianist should experience no technical difficulties when playing this scale, and should find the task "easy." As such, two hypotheses are proposed:

Hypothesis SC-LO (scale - low): The levels of active muscle stiffness and cocontraction that are measured will be low.

Hypothesis SC-NC (scale - no change): There will be no significant change in the level of active muscle stiffness and co-contraction during the exercise, given that the participants recruited for this study are experienced pianists.

To test hypothesis SC-LO, the mean active muscle stiffness and co-contraction will be calculated for the length of the scale, excluding the last note, which is held for much longer than the notes making up the scale. If the mean is below $15 \% \mathrm{MVC}$, it can be concluded that active muscle stiffness (activity of the FCU, the ECU, the FDS and the EDC) and co-contraction (CC1 and CC2) remained low.

To test hypothesis SC-NC, the mean EMG level of the first five seconds of the scale will be compared to the mean EMG level of the last five seconds of the scale, excluding the duration of the last note, to see if there is a significant change in active muscle stiffness and co-contraction. 
The EMG data collected while the last note of the scale was played is not included in the analysis because this note was played for much longer than any of the other notes making up the scale. Including the last note in the analysis would introduce bias into the results because participants held the last notes for varying lengths of time. Additionally, participant E010 continuously played the $\mathrm{C}$ major scale more than three times, and hence did not hold the C3 key at the end of the third (last) descending segment.

\subsubsection{EMG Results of C Major Scale}

EMG files were collected (as described in Chapter 3), processed, trimmed and sectioned (as described in Chapter 4). EMG data for each of the four muscles measured - the FCU, ECU, FDS and EDC - were plotted versus time, and co-contraction data (CC1 and CC2) were plotted versus time. Participant E002's results are shown in Figure. 5.1 (active muscle stiffness) and Figure. 5.2 (co-contraction).

The results of E002 are typical of how most participants performed. (For results of all other participants, see Appendix H.) Mean active muscle stiffness appears to fluctuate between $20 \%$ and $50 \%$ MVC depending on which muscle is being measured. This is much higher than 15\% MVC, the proposed definition of low active muscle stiffness and low co-contraction. Based on these observations, muscle stiffness and co-contraction are not low while playing the $\mathrm{C}$ major scale in parallel motion at 108 bpm, and hypothesis NC-LO is rejected.

Based on visual inspection of the EMG results, E002 appears to exhibit higher levels of active muscle stiffness and co-contraction during the ascending segment of the scale than during the descending segment. This is not unexpected. Different finger and hand motions are used to play the ascending and descending segments of a scale. 

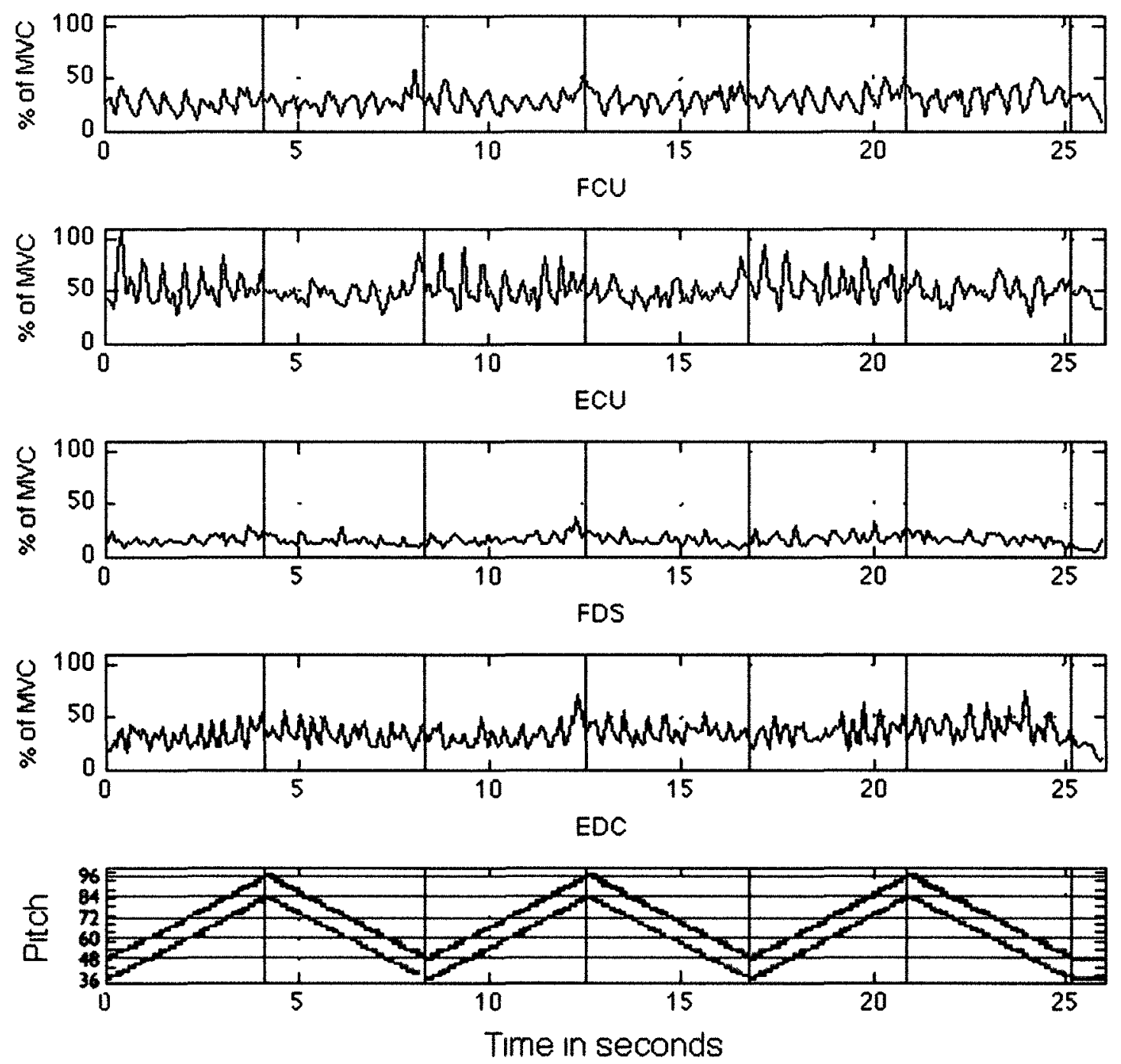

Figure 5.1: Participant E002's results the upper plots show active muscle stiffness, expressed as a percentage of maxımal voluntary contractıon, whıle playıng the $\mathrm{C}$ major scale in parallel motion, ascending and descending for four muscles of the right forearm the FCU, the ECU, the FDS and the EDC The corresponding MIDI data (note on, note off) are shown on the lower plot 

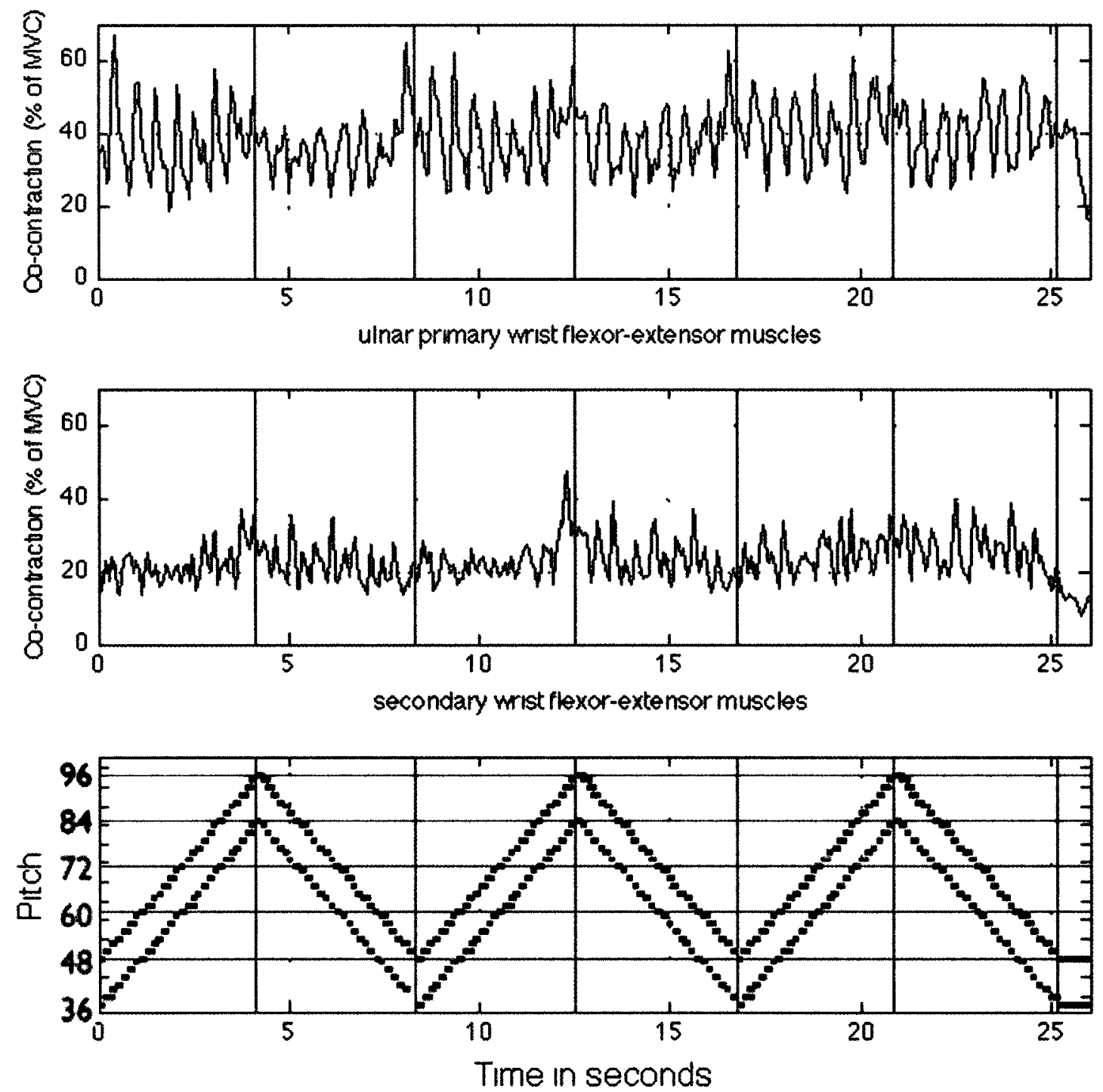

Figure 5.2: Partıcipant E002's results the upper plots show levels of muscular cocontraction, expressed as a percentage of maximal voluntary contraction, while playıng the $\mathrm{C}$ major scale in parallel motıon, ascending and descending The corresponding MIDI data (note on, note off) are shown on the lower plot 
When playing the ascending segment of the C major scale, the "thumb under" motion of the right thumb is used, which causes some radial deviation of the right hand [55]; when playing the descending segment of the $\mathrm{C}$ major scale the middle and ring fingers of the right hand (i.e. fr3 and fr4, respectively) use the "finger over" motion, which causes some radial deviation as the finger crosses over the thumb, followed by ulnar deviation as the descending notes are played [55]. Consequently, variable levels of active muscle stiffness and co-contraction in forearm muscles are expected according to the direction of the scale, and the proposed method to test hypothesis SC-NC cannot be used. For example, participant E002 uses $82.5 \%$ of the first five seconds to play the first ascending segment and uses $17.5 \%$ of the first five seconds to play the first descending segment; $13.4 \%$ of the last five seconds (excluding the last note) is spent playing the third ascending segment and $86.6 \%$ of the last five seconds is spent playing the third descending segment. If active muscle stiffness and co-contraction vary with the direction of the scale, comparing the first five seconds to the last five seconds will not correctly indicate if active muscle stiffness and co-contraction are changing because it is not possible to say which factor the change is attributable to: the direction of the scale or an increase in muscle activity over time.

A possible solution to this problem would be to pool the first ascending and descending segments, pool the third ascending and descending segments, and compare both pooled segments to each other. The problem with this method is that pooling ascending and descending segments together might mask any changes that are taking place. For example, if the level of muscle activity goes up from ascending segment 1 (A1) to ascending segment $3(A 3)$, but the level of muscle activity goes down from descending segment 1 (D1) to descending segment 3 (D3), this may be masked when $A 1-D 1$ results are compared to $A 3-D 3$ results.

An alternative to the two above methods was used. First it was determined if there 
were an actual difference between pooled ascending segments and pooled descending segments. To do this, the EMG collected during the playing of the scale was split into six sections: $A 1$ - ascending segment $1, D 1$ - descending segment $1, A 2-$ ascending segment 2,D2- descending segment 2, A3 - ascending segment 3 and $D 3$ - descending segment 3. $A 1, A 2$ and $A 3$ were pooled to give $A ; D 1, D \mathscr{2}$ and $D 3$ were pooled to give $D$. The means of $A$ and $D$ were compared to each other using a one-way analysis of variance - or ANOVA - with the alpha level of probability set at 0.05 for each measure of active muscle stiffness and co-contraction for each participant. The summary of the ANOVA is shown in Table 5.2. The means of the pooled EMG data for the ascending segments and descending segments can be found in Appendix I. Table I.1 shows the means of the FCU, ECU, FDS and EDC; Table I.2 shows the means of the two measures of co-contraction.

It should be noted that the results of participant E008 were not included in the analysis because this participant's MIDI file was not recorded. Without the MIDI file, it is difficult to identify precisely where the ascending and descending segments of the scale began and ended. Also, it appears there was a problem with the FCU data while participant E006 played the first ascending and first descending segments of the scale. These data have been left out of the analysis. Thus, when $A$ is compared to $D$ for participant E006's FCU, $A$ comprises most of $A \mathscr{2}$ and all of $A 3$ (but not $A 1$ ) and $D$ comprises $D 2$ and $D 3$ (but not $D 1$ ).

According to Table 5.2, there are significant differences between the ascending and descending segments of the scale. The level of activity of the FCU is generally higher during the descending segment or not significantly different from the ascending segment of the scale. Conversely, the level of activity of the FDS is generally higher 
Table 5.2: Summary of one-way ANOVA tests (with the alpha level of probability set at 0.05) comparing mean active muscle stiffnesses of the FCU, ECU, FDS and EDC and mean co-contraction levels ( $\mathrm{CC} 1$ and $\mathrm{CC} 2$ ) in ascending and descending segments of the $\mathrm{C}$ major scale for participants in this study. The total number of participants where the mean of the pooled ascending segments is significantly larger than the mean of the pooled descending segments is indicated in the column titled $A>D$. The total number of participants where the mean of the pooled ascending segments is significantly smaller than the mean of the pooled descending segments is indicated in the column titled $A<D$. The total number of participants where the means were not significantly different is indicated in the column titled $A=D$.

\begin{tabular}{|c|c|c|c|}
\hline & $\mathbf{A}>\mathbf{D}$ & $\mathbf{A}<\mathbf{D}$ & $\mathbf{A}=\mathbf{D}$ \\
\hline \hline FCU & 1 & 3 & 4 \\
\hline ECU & 4 & 2 & 2 \\
\hline FDS & 7 & 0 & 1 \\
\hline EDC & 3 & 3 & 2 \\
\hline CC1 & 1 & 1 & 6 \\
\hline CC2 & 5 & 0 & 3 \\
\hline
\end{tabular}


during the ascending segment than during the descending segment of the scale. Overall, when only active muscle stiffness results are considered, in more than half of all cases the ascending segment of the scale is significantly larger than the descending segment. When only the co-contraction results are considered, the ascending and descending segments of the scale are not significantly different in more than half of all cases.

Next, scale segments $A 1, A 2$ and $A 3$ were compared by means of a one-way ANOVA to determine if there were any significant change in active muscle stiffness or co-contraction from the beginning to the end of the scale; the same approach was used for scale segments D1, D2 and D3. The ANOVA identified where ascending segments of the scale had different means; it did the same for the descending segments. Where ANOVA indicated that the means of the ascending segments differed (similarly, where ANOVA indicated that the means of the descending segments differed), the TukeyKramer method was used to show which pairs of means differed. The Tukey-Kramer method calculates the minimum significant difference (or MSD) for each pair of means. If the MSD is smaller than the observed difference of a pair of means, the pairs are significantly different. The summary of the ANOVA and comparison of the means using the Tukey-Kramer method is shown in Table 5.3 for segments $A 1, A 2$ and $A 3$. The results for segments D1,D2 and D3 are shown in Table 5.4. The alpha level of probability was set at 0.05 . The ANOVA and Tukey-Kramer method were performed using a spreadsheet from McDonald's Handbook of Brological Statıstzcs [56]. The means of the EMG data for the A1, A2, A3, D1, D2 and D3 segments can be found in Appendix J. Tables J.1 and J.2 show the mean active muscle stiffness of the FCU, ECU, FDS and EDC; Tables J.3 and J.4 show the means of CC1 and CC2.

In the ascending segments where the means were not equal, there was an increase 
Table 5.3: Summary of pairwise comparisons of mean active muscle stiffness and co-contraction levels in $\mathrm{C}$ major scale segments $A 1, A 2$ and $A 3$ using the TukeyKramer method (with the alpha level of probability set at 0.05 ) for participants in this study. The paired comparisons were: A1 to A2, A1 to A3, A2 to A3. Where means of pairs were not significantly different, the results for all participants were pooled in the column $\mathrm{A} 1=\mathrm{A} 2, \mathrm{~A} 1=\mathrm{A} 3, \mathrm{~A} 2=\mathrm{A} 3$. The total number of participants where the means of a pair were significantly different is given in columns $\mathrm{A} 1 \neq \mathrm{A} 2, \mathrm{~A} 1 \neq \mathrm{A} 3$ and $\mathrm{A} 2 \neq \mathrm{A} 3$. In all cases where the means were not equal, mean $\mathrm{A} 1<$ mean $\mathrm{A} 2$ and mean $\mathrm{A} 1<$ mean $\mathrm{A} 3$. In all cases except one (denoted by $\mathrm{a}^{*}$ ), mean A2 $<$ mean A3.

\begin{tabular}{|c|c|c|c|c|}
\hline & \multicolumn{4}{|c|}{ Ascending } \\
\hline & $\begin{array}{l}\text { A1=A2, } \\
\text { A1=A3, } \\
\text { A2=A3 }\end{array}$ & A1 $\neq$ A2 & A1 $\neq$ A3 & A2 $\neq$ A3 \\
\hline \hline FCU & 17 & 2 & 3 & $1+1^{*}$ \\
\hline ECU & 24 & - & - & - \\
\hline FDS & 20 & - & 2 & 2 \\
\hline EDC & 21 & 1 & 2 & - \\
\hline CC1 & 20 & 1 & 3 & - \\
\hline CC2 & 16 & 1 & 4 & 3 \\
\hline
\end{tabular}


Table 5.4: Summary of pairwise comparisons of mean active muscle stiffness and co-contraction levels in C major scale segments $D 1, D 2$ and $D 3$ using the TukeyKramer method (with the alpha level of probability set at 0.05) for participants in this study. The paired comparisons were: D1 to D2, D1 to D3, D2 to D3. Where means of pairs were not significantly different, the results for all participants were pooled in the column $\mathrm{D} 1=\mathrm{D} 2, \mathrm{D} 1=\mathrm{D} 3, \mathrm{D} 2=\mathrm{D} 3$. The total number of participants where the means of a pair were significantly different is given in columns $\mathrm{D} 1 \neq \mathrm{D} 2, \mathrm{D} 1 \neq \mathrm{D} 3$ and $\mathrm{D} 2 \neq \mathrm{D} 3$. In all cases where the means were not equal, mean D1 < mean D2, mean D1 < mean D3 and mean D2 < mean D3.

\begin{tabular}{|c|c|c|c|c|}
\hline & \multicolumn{4}{|c|}{ Descending } \\
\hline & $\begin{array}{l}\text { D1=D2, } \\
\text { D1=D3, } \\
\text { D2=D3 }\end{array}$ & D1 $\neq$ D2 & D1 $\neq$ D3 & D2 $\neq$ D3 \\
\hline \hline FCU & 19 & - & 3 & 2 \\
\hline ECU & 22 & - & 1 & 1 \\
\hline FDS & 23 & - & 1 & - \\
\hline EDC & 19 & 2 & 3 & - \\
\hline CC1 & 19 & - & 4 & 1 \\
\hline CC2 & 19 & 1 & 4 & - \\
\hline
\end{tabular}


in active muscle stiffness and co-contraction: in all cases, mean A1 < mean A2 and mean $\mathrm{A} 1<$ mean A3; in all cases except one (denoted by a * in Table 5.3), mean A2 $<$ mean A3. The same holds true for the descending segments: mean D1 $<$ mean D2, mean D1 < mean D3 and mean D2 < mean D3 (see Table 5.4).

In the majority of cases, ascending segments do not have means that are statistically different (see Table 5.3); the same is true of the descending segments (see Table 5.4). The values in the column "A1=A2, $\mathrm{A} 1=\mathrm{A} 3, \mathrm{~A} 2=\mathrm{A} 3$ " of Table 5.3 are much higher than the values in columns $\mathrm{A} 1 \neq \mathrm{A} 2, \mathrm{~A} 1 \neq \mathrm{A} 3$ and $\mathrm{A} 2 \neq \mathrm{A} 3$ of Table 5.3 even if these columns were to be combined. Similarly, in Table 5.4, the values in the column "D1=D2, D1=D3, D2=D3" are greater than the values in columns columns D1 $\neq \mathrm{D} 2$, $\mathrm{D} 1 \neq \mathrm{D} 3$ and $\mathrm{D} 2 \neq \mathrm{D} 3$. This indicates that active muscle stiffness and co-contraction are not changing (neither increasing nor decreasing) from the first segment of the scale to the third segment of the scale, thus confirming hypothesis SC-NC.

\subsection{Discussion}

\subsubsection{Execution of C Major Scale}

To evaluate the execution of the $\mathrm{C}$ major scale the note rate, expressed in notes per second played by the right hand, was reported. Any added or repeated notes might increase the note rate, assuming the left hand played the scale correctly. Similarly, if notes were skipped, this might be reflected by a lower note rate. The note rate does not reflect where note accuracy errors occurred, but it provides an adequate indication of how the participant performed the task. 


\subsubsection{EMG Results and Hypothesis SC-LO}

Initially it was hypothesized that participants would exhibit low levels of active muscle stiffness and co-contraction while playing three repetitions of the $\mathrm{C}$ major scale, since the $\mathrm{C}$ major scale is generally the first scale learned by piano students. This was not observed. The majority of the time (in approximately in $95 \%$ of cases) the levels of mean active muscle stiffness and co-contraction were above $15 \%$. These high levels might be the outcome of the normalization methods used, specifically the choice of exercises to elicit a MVC and the calculation of the MVC. Normalization will be discussed in Chapter 8 .

It was hypothesized by Vant that increasing the note rate would lead to an increase in wrist stiffness, and this criterion was incorporated by Ann Southam in her composition of Piano Lab. If the Ann Southam piece is played at the correct tempo and according to the indicated time signatures, the piece reaches a maximum of four notes

per second, and it is expected that active muscle stiffness and co-contraction will be high when this maximum is reached. When the $\mathrm{C}$ major scale is played according to the instructions specified in this study, the note rate is 7.2 notes per second. If note rate affects levels of active muscle stiffness and co-contraction, this may explain why active muscle stiffness and co-contraction were not low during the $\mathrm{C}$ major scale task of this study. Thus, a new hypothesis is proposed: note rate during scales is directly related to active muscle stiffness and co-contraction levels. To test this hypothesis, it would be worthwhile to conduct a series of experiments where participants play the C major scale at a variety of tempos corresponding to one, two, three and four notes per second. This study could possibly examine note rates as high as eight notes per second, which is the equivalent of $120 \mathrm{bpm}$ when the scale is played in sixteenth notes. The order in which the scales would be played could be varied so that the order would not affect the outcome of the results. The participants could also be given more time 
between tasks (i.e. scales) to relax in order to reduce the effects of fatigue.

It is also possible that low levels of active muscle stiffness and co-contraction typically were not observed during the scales because the scales spanned four octaves. Given that a white key on a piano has a width of $23 \mathrm{~mm}$, the hand of a pianist must travel $644 \mathrm{~mm}$ when playing the four ascending octaves of a piano. This is referred to as hand excursion. A hand excursion of approximately $322 \mathrm{~mm}$ (half $644 \mathrm{~mm}$ ) across the torso is somewhat large when compared to everyday tasks, such as typing. To test if hand excursion affects active muscle stiffness and co-contraction levels, a study could be conducted where the participants are asked to play two-octave and four-octave scales at a set tempo.

\subsubsection{EMG Results and Hypothesis SC-NC}

Initially it was hypothesized that participants would not experience a significant change in active muscle stiffness and co-contraction while playing the $\mathrm{C}$ major scale. A visual inspection of the EMG plots shows that there appear to be differences in patterns according to the direction of the scale (i.e. whether the scale is ascending or descending). To test if directionality affected mean active muscle stiffness and co-contraction levels, the three continuous repetitions of the scale were split into six segments: three ascending and three descending. The data from ascending segments were pooled, as were the data from the descending segments. ANOVA revealed that mean active muscle stiffnesses of the FDS during the ascending and descending segments were significantly different in most cases, but that mean active muscle stiffness of the FCU did not change significantly between ascending and descending portions of the scale. This could possibly be because of the different movements during the ascending and descending segments of the scale.

These movements are described in great detail in Wristen's paper on a proposal of 
theoretical procedures for biomechanical analysis of piano technique [55]. The MCP flexion, which occurs in order to depress a piano key, is effectively accomplished by the FDS. During the descending segment of the $\mathrm{C}$ major scale in the right hand, pronation and deviation movements throw the third (or fourth) finger(s) over the thumb [55]. This is called the "finger over" action of the third or fourth fingers. Since it raises the third (or, at times, the fourth) finger, this may add additional force due to gravity that does not occur in the ascending scale of the right hand. This may explain why the mean active muscle stiffness is significantly greater during the ascending segment of the scale than in the descending segment of the scale.

The lateral motions of the wrist to adjust the hand so it is properly positioned over the keys are a combination of ulnar and radial deviations. During the ascending segment of $\mathrm{C}$ major scale of the right hand, there is increasingly less radial deviation [55]. During the descending segment of the the $\mathrm{C}$ major scale there is increasingly more ulnar deviation of the right hand as it moves towards the position it started from, but there is also radial deviation during "finger over" motion of the third and fourth fingers [55]. Perhaps for this reason, there is no obvious pattern with respect to scale direction and active muscle stiffness in the FCU or ECU. Since co-contraction of muscles with their primary action at the wrist (or CC1) is a function of the active stiffness levels of the FCU and ECU, and because there is no obvious pattern in active muscle stiffness of the FCU and ECU with respect to scale direction, this is a logical explanation for the lack of significant difference in CC1 between ascending and descending segments of the scale.

In addition to comparing mean active muscle stiffness and co-contraction levels in the ascending and descending segments of the scale, the three ascending segments were compared to one another, and the three descending were compared to one another to determine if there were significant changes in mean active muscle stiffness and 
co-contraction from the beginning to the end of the scale. It was found that in most cases, mean active muscle stiffness and co-contraction did not change from the start to the end of the scale, thus confirming hypothesis SC-NC.

The actions of the FCU and ECU produce ulnar deviation in addition to the flexion and extension actions at the wrist. It would be worthwhile to record the activities of muscles producing radial deviation to determine if the mean active muscle stiffnesses during the ascending and descending segments of the scale were significantly different.

In future studies, the position of the pianist with respect to the keyboard should be analyzed. As the hands of the pianist travel up and down the keyboard, the pianist might choose to adjust his or her position with respect to the keyboard. This could potentially affect the amount of ulnar and radial deviation, thus producing changes in active muscle stiffness (and by extension, the co-contraction) of muscles controlling these movements that might vary with scale direction.

\subsection{Outcomes of Hypothesis Testing}

In this chapter, two hypotheses were proposed:

1. Hypothesis SC-LO: Active muscle stiffness and co-contraction levels will be low while playing the $\mathrm{C}$ major scale.

This hypothesis was rejected.

2. Hypothesis SC-NC: Active muscle stiffness and co-contraction levels will not change significantly from the beginning to the end of the $\mathrm{C}$ major scale.

This hypothesis was confirmed. 


\section{Chapter 6}

\section{Task \#2: Results and Discussion}

\subsection{Results of Task \#2: Triad Exercises}

The participants were asked to play two triad exercises, which are shown in Figure. 6.1. These particular exercises were adapted from Dohnányi's Essentıal Finger

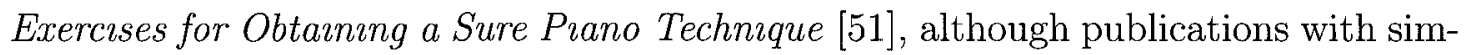
ilar exercises exist. There is some speculation in the piano community that these exercises, which emphasize finger independence, help to develop a secure playing technique.

The triad exercises were demonstrated on the piano to the participant by the researcher, who held the alternating notes when demonstrating the task. An explanation of holdıng is given in Section 6.1.1. Participants did not play from the musical scores shown in Figure. 6.1. Participants were told to play as fast as he or she could, and did not play with a metronome.

The first exercise required that the participant hold down fingers one (fr1), three (fr3) and five (fr5) of the right hand while alternately depressing keys with fingers two (fr2) and four (fr4) thirty times. This exercise is referred to as TR1. The second exercise required that the participant hold down piano keys with fr 1 , fr2 and fr5 while 


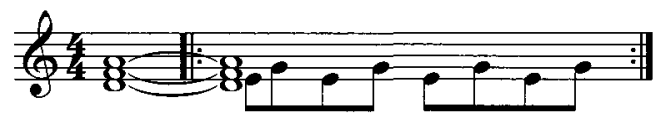

(a) TR1

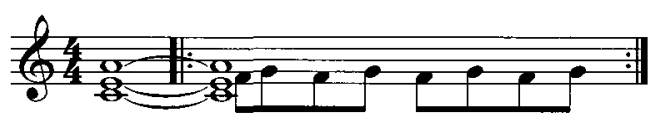

(b) TR2

Figure 6.1: Musical score for (a) TR1 and (b) TR2. It should be noted that while the score indicates to play the alternating notes eight times (when the repeats are followed), participants were asked to play the alternating notes thirty times.

alternately depressing keys with fr3 and fr4 thirty times. This exercise is referred to as TR2.

\subsubsection{Execution of Triad Exercises}

Generally speaking, participants were more successful in executing TR2. The results of several participants in this study were eliminated from the analysis, as these people did not complete the task as required. If one of the fingers that was to remain depressed throughout the exercise (fr1, fr3 and fr5 in TR1; fr1, fr2 and fr5 in TR2) lifted off one of the keys for $10 \%$ or more of the time, that participant's results were eliminated. The following results of the triad exercises were eliminated from the analysis:

- E002 TR1: Did not keep fr1, fr3 and fr5 depressed for the entire duration of the exercise. E002 lifted fr1 three times during TR1; lifted fr3 six times during TR1; lifted fr5 three times during TR1. From start to finish, fr1 was lifted almost $25 \%$ of the time, fr3 was lifted almost $50 \%$ of the time, and fr5 was lifted approximately $10 \%$ of the time.

- E005 TR1: Did not keep fr3 depressed for the entire duration of the exercise. E005 lifted fr3 approximately 7.5 seconds after beginning TR1, and did not depress fr3 again for the remainder of the exercise. 
- E006 TR2: Did not depress fr1 for the entire duration of the exercise.

- E007 TR1: Did not keep fr1, fr3 and fr5 depressed for the entire duration of the exercise. E007 lifted fr1 two times during TR1; lifted fr3 seven times; lifted fr5 three times. From start to finish, fr1 was lifted almost $2.0 \%$ of the time, fr3 was lifted almost $25 \%$ of the time, and fr 5 was lifted almost $3.2 \%$ of the time.

At the end of the exercises, most participants noted that they felt some discomfort while performing the tasks, and that TR1 was more difficult and required more concentration than TR2. Many observed that they felt stiffer and experienced more discomfort when executing TR1 than TR2.

In executing TR1 and TR2, participants used two strategies. One subset of participants played the alternating notes in a detached (or staccato) manner, so that the keys were depressed for only short lengths of time; this strategy will be referred to as tapping. Another subset of participants played the alternating notes in a more legato (or smooth) manner, holding the alternating notes for longer times; this strategy will be referred to as holding. Holding was classified as depressing the alternating notes for 0.15 seconds or longer on average; tapping was classified as depressing the alternating notes for less than 0.15 seconds. The bottom plots of Figure. 6.2 show the MIDI data of a participant who held alternating notes; the bottom plot of Figure. 6.4(a) shows the MIDI data of a participant who tapped alternating notes. After the participants were eliminated on account of not properly executing the task, there remained six participants who correctly executed TR1 and eight participants who corrected executed TR2. Of these remaining participants, four participants held and two participants tapped the alternating notes in TR1; five participants held and three participants tapped the alternating notes in TR2. The results are shown in Table 6.1. 
Table 6.1: Mean duration of alternating notes in TR1 and TR2, measured in seconds, and associated strategy used to play alternating notes. A $\dagger$ indicates that the participant's results are not valid, as that person did not execute the task properly.

\begin{tabular}{|c|c|c|c|c|c|}
\hline & \multicolumn{2}{|l|}{ TR1 } & \multicolumn{2}{|l|}{ TR2 } \\
\hline & & $\begin{array}{l}\text { Average duration of } \\
\text { alternating notes (s) }\end{array}$ & Strategy & $\begin{array}{l}\text { Average duration of } \\
\text { alternating notes (s) }\end{array}$ & Strategy \\
\hline \multirow{9}{*}{ 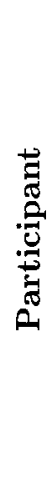 } & E002 & 0.727 & holding $\dagger$ & 0.392 & holding \\
\hline & E003 & 0.204 & holding & 0.346 & holding \\
\hline & E004 & 0.188 & holding & 0.155 & holding \\
\hline & E005 & 0.101 & tapping $\dagger$ & 0.085 & tapping \\
\hline & E006 & 0.150 & holding & 0.379 & holding $\dagger$ \\
\hline & E007 & 0.431 & holding $†$ & 0.358 & holding \\
\hline & E008 & 0.088 & tapping & 0.336 & holding \\
\hline & E009 & 0.163 & holding & 0.109 & tapping \\
\hline & E010 & 0.111 & tapping & 0.144 & tapping \\
\hline
\end{tabular}

\subsubsection{Hypotheses — Triad Exercises}

Holding down three piano keys with the fingers, while alternately depressing two other piano keys with the remaining two fingers is a challenging task. It relies on finger co-ordination, which may call upon intrinsic muscles of the hand in addition to using the extrinsic muscles of the forearm such as the four muscles measured in this study. Consequently, two hypotheses are proposed:

Hypothesis TR-SC (triads - significant change): There will be a significant increase in active muscle stiffness and co-contraction throughout the exercise.

Hypothesis TR-HI (triads - high): The level of active muscle stiffness and cocontraction will be high.

To test hypothesis TR-SC, the means of the first and last three seconds of active muscle stiffness and co-contraction data will be compared to determine if there is a 
significant change in active muscle stiffness and co-contraction from the start to the end of the exercise. It is expected that the mean of the last three seconds of EMG data will be significantly higher than the mean of the first three seconds of EMG data.

Hypothesis TR-HI will be confirmed if the means of the first and last three seconds of active muscle stiffness and co-contraction data are greater than 15\% MVC.

\subsubsection{EMG Results of Triad Exercises}

Before beginning testing of these hypotheses, the EMG files were processed and trimmed according to the procedure described in Section 4.2.2 in order to eliminate EMG data that were recorded before the first MIDI event. EMG data of the FCU, ECU, FDS and EDC were plotted versus time to visualize active muscle stiffness throughout the exercises; additionally, co-contraction data were plotted versus time. Active muscle stiffness and co-contraction TR2 results of participant E002 are shown in Figure. 6.2. Plots showing active muscle stiffness and co-contraction for all participants can be found in Appendix $\mathrm{K}$ and Appendix L, respectively.

Next, TR1 and TR2 EMG files were divided into five segments and sequentially numbered. The divisions were as follows:

- Segment 1: rows 1 to 30 ,

- Segment 2: rows 31 to 80 ,

- Segment 3: rows 81 to end-81,

- Segment 4: rows end-80 to end-31,

- Segment 5: rows end-30 to end. 

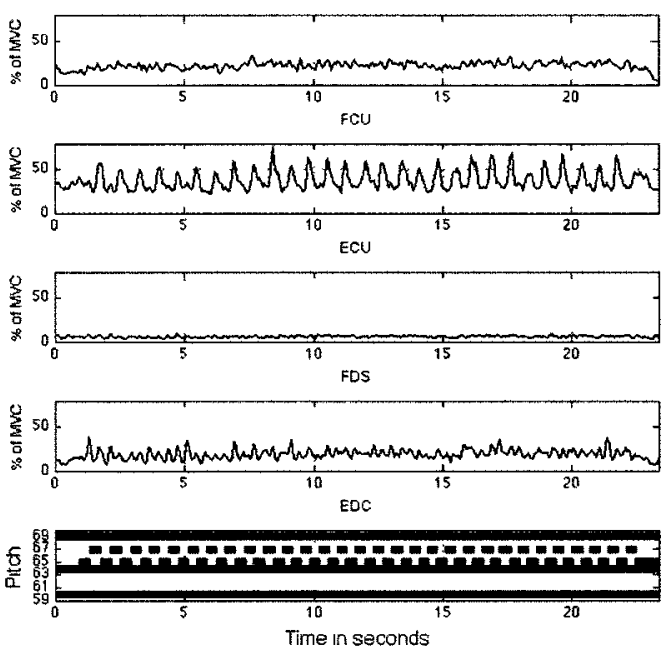

(a) active muscle stiffness - TR2
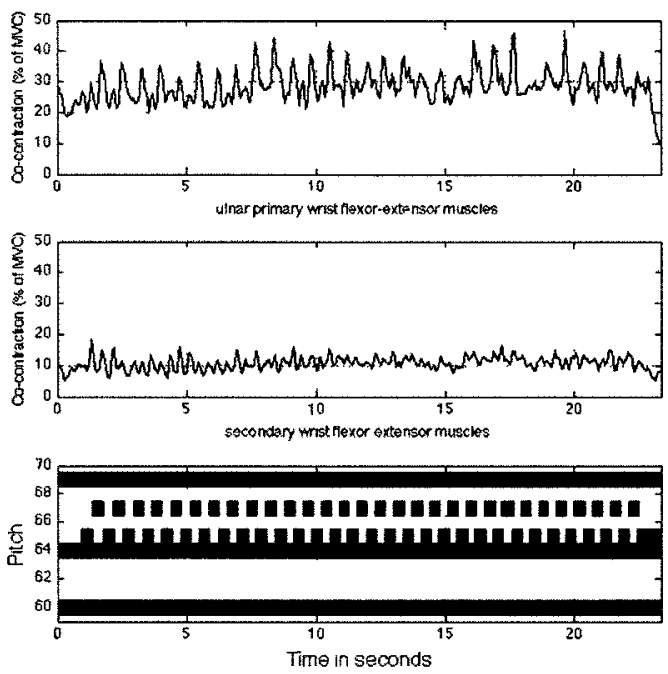

(b) co-contraction - TR2

Figure 6.2: Participant E002's results: the upper plots of (a) show active muscle stiffness for the FCU, ECU, FDS and EDC of the right forearm, and the upper plots of (b) show levels of muscular co-contraction while playing TR2. Active muscle stiffness and co-contraction are expressed as a percentage of maximal voluntary contraction, while playing the second triad exercise (TR2). The corresponding MIDI data (note on, note off) are shown on the bottom plots of (a) and (b).

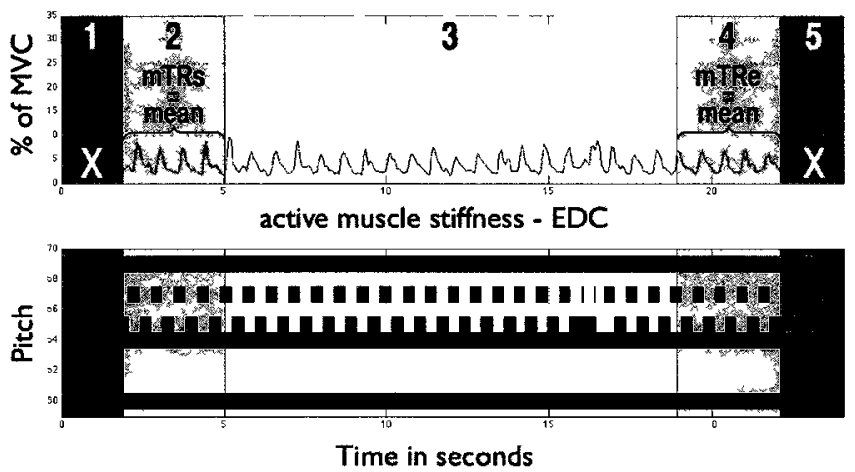

Figure 6.3: Participant E008's active muscle stiffness of the EDC and corresponding MIDI data from TR2, showing the segments of the file that were discarded (segments 1 and 5) and the segments that were compared (segments 2 and 4). 

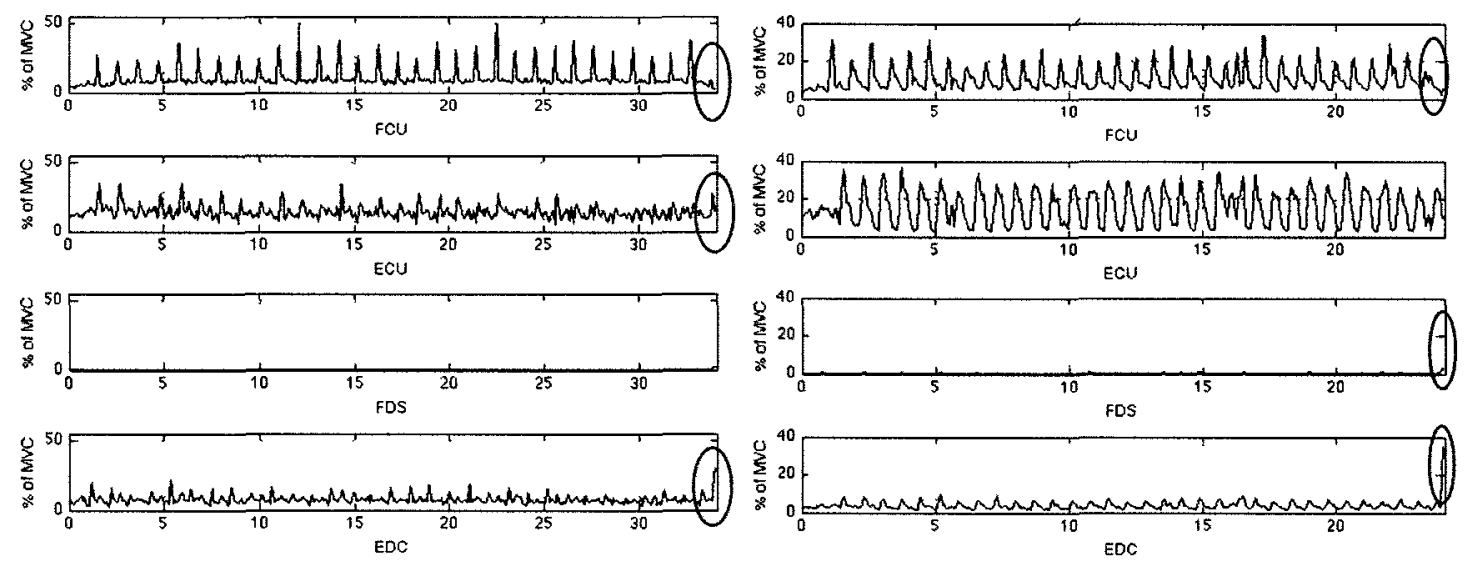

\section{홀}

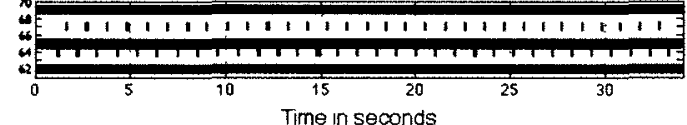

(a) TR1

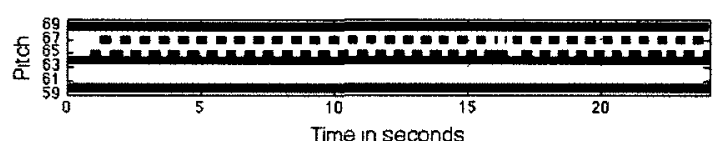

(b) TR2

Figure 6.4: Participant E008's results for both triad exercises, TR1(a) and TR2(b). The upper plots show active muscle stiffness for the FCU, ECU, FDS and EDC of the right forearm, expressed as a percentage of maximal voluntary contraction. The EMG pattern changes at the end of the exercise. This is indicated by the black ovals. The corresponding MIDI data (note on, note off) are shown on the lower plot.

These divisions are shown in Figure. 6.3.

As can be seen from Figure. 6.2, as well as the figures in Appendices $\mathrm{K}$ and L, participants took variable lengths of time to begin playing the alternating notes after having depressed the three keys that were held for the duration of the exercise. They began playing the alternating notes between $0.414 \mathrm{~s}$ and $1.817 \mathrm{~s}$ after depressing the held keys, and on average, participants depressed the three held keys for $1.3 \mathrm{~s}$ before beginning to play the alternating notes. The pattern of the EMG data also appears to change near the end of the exercise, and may be partly attributable to participants finishing the exercise and lifting their hand off the piano keys. This is especially noticeable in participant E008's data (see Figure. 6.4). For these reasons, segments 1 and 5, each corresponding to $1.89 \mathrm{~s}$ of data, were discarded. 


\section{Hypothesis TR-SC}

As identified in Section 6.1.2, it was expected that from the start to the end of TR1 and TR2 there would be a significant increase in active muscle stiffness and cocontraction. The means of the entries in each of segments 2 and $4^{1}$ were calculated. These means, mTRs and mTRe, respectively, were compared using Student's t-test to determine if they were significantly different. (The resulting P-values can be found in Table M.1 of Appendix M.) The values of mTRs and mTRe are presented in Tables 6.2 and 6.3. Table 6.2 shows the mTRs and mTRe values of the active muscle stiffness data; Table 6.3 gives the mTRs and mTRe values of the co-contraction data. A $※$ indicates where mTRs and mTRe are significantly different; $※$ is placed next to the mean that is larger. In most cases, where the means are significantly different, mTRe is larger than mTRs. In TR1, there is only one case (participant E008) where mTRe is significantly smaller than mTRs. In TR2, there are two cases where mTRe is significantly smaller than mTRs: participants E003 and E005.

The results presented in Tables 6.2 and 6.3 are summarized in Table 6.4. Table 6.4 shows that there are more instances where mTRs $<$ mTRe than mTRs $>$ mTRe, but that the number of instances where mTRs $<$ mTRe and mTRs $=$ mTRe are about equal. This means that from the start to the end of the triad exercise, active muscle stiffness and co-contraction increased more often than they decreased, but that active muscle stiffness and co-contraction increased in approximately the same number of cases as where they did not change significantly.

\footnotetext{
${ }^{1}$ Segments 2 and 4 each correspond to $3.15 \mathrm{~s}$ of data.
} 
Table 6.2: Mean actıve stıffnesses of the FCU, ECU, FDS and EDC in segments 2 (mTRs) and 4 (mTRe) of TR1 and TR2 A ※ indicates that the means are significantly different, and is placed next to the larger mean A $\dagger$ indicates that the participant's results are not valıd because they did not execute the task correctly, and therefore are not included in the table

\begin{tabular}{|c|c|c|c|c|c|c|c|c|c|c|}
\hline & \multicolumn{8}{|c|}{ Active Muscle Stiffness } \\
\hline & & & \multicolumn{4}{|c|}{ TR1 } & \multicolumn{4}{|c|}{ TR2 } \\
\hline & & & FCU & ECU & FDS & EDC & FCU & ECU & FDS & EDC \\
\hline \multirow{18}{*}{ 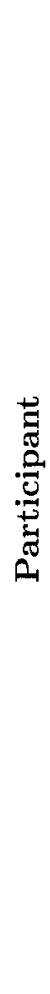 } & FOn? & mTRs & & & & & 2145 & 3367 & 539 & 1722 \\
\hline & & mTRe & 1 & 1 & 1 & 1 & $2473 ※$ & $3852 ※$ & $610 \%$ & $2169 \times$ \\
\hline & \multirow{2}{*}{ E003 } & mTRs & 3043 & 6051 & 5218 & 5612 & 2229 & $6941 ※$ & 3114 & 5333 \\
\hline & & mTRe & $3598 \%$ & 6555 & 5869 & 6030 & 2100 & 5100 & 2859 & 4853 \\
\hline & \multirow{2}{*}{ E004 } & mTRs & 3473 & 3591 & 721 & 2822 & 3221 & 4045 & 634 & 3147 \\
\hline & & mTRe & $4322 \approx$ & $4732 *$ & 761 & 4054 ※ & 3934 ※ & 3982 & $735 \%$ & 3434 \\
\hline & \multirow{2}{*}{ E005 } & mTRs & \multirow{2}{*}{$\dagger$} & \multirow{2}{*}{$\dagger$} & \multirow{2}{*}{$\dagger$} & \multirow{2}{*}{$\dagger$} & 3269 & 3481 ※ & 1283 & 3031 \\
\hline & & mTRe & & & & & 3303 & 3117 & 1319 & 3307 \\
\hline & \multirow{2}{*}{ E006 } & mTRs & 1859 & 3755 & 1311 & 1454 & \multirow{2}{*}{$\dagger$} & \multirow{2}{*}{$\dagger$} & \multirow{2}{*}{$\dagger$} & \multirow{2}{*}{$\dagger$} \\
\hline & & mTRe & $2965 \nsim$ & $4343 \%$ & $1438 \%$ & $1762 \approx$ & & & & \\
\hline & \multirow{2}{*}{ E007 } & mTRs & \multirow{2}{*}{$\dagger$} & \multirow{2}{*}{$\dagger$} & \multirow{2}{*}{ t } & \multirow{2}{*}{$\dagger$} & 2140 & 2405 & 1191 & 921 \\
\hline & & mTRe & & & & & $2331 ※$ & $2718 \approx$ & 1265 & $1171 \times$ \\
\hline & \multirow{2}{*}{ E008 } & mTRs & 974 & $1676 *$ & 046 & 800 & 1162 & 1488 & 047 & 383 \\
\hline & & mTRe & 1095 & 1278 & 047 & 712 & 1127 & 1558 & 044 & 376 \\
\hline & \multirow{2}{*}{ E009 } & mTRs & 1768 & 6047 & 3264 & 5450 & 1730 & 4320 & 2268 & 3848 \\
\hline & & mTRe & 1944 & 6097 & $3753 *$ & 5999 & $2100 \%$ & $5306 \%$ & $2752 \approx$ & $4644 ※$ \\
\hline & \multirow{2}{*}{ E010 } & mTRs & 4550 & 3913 & 2657 & 1525 & 3963 & 4399 & 1163 & 1253 \\
\hline & & mTRe & 4405 & 3971 & 2664 & 1569 & $4491 ※$ & 4131 & 1167 & 1381 \\
\hline
\end{tabular}


Table 6.3: Mean co-contraction levels (CC1 and CC2) in segments 2 (mTRs) and 4 (mTRe) of TR1 and TR2. A $※$ indicates that the means are significantly different, and is placed next to the larger mean. $A \dagger$ indicates that the participant's results are not valid because they did not execute the task correctly, and therefore are not included in the table.

\begin{tabular}{|c|c|c|c|c|c|c|}
\hline & \multicolumn{4}{|c|}{ Co-contraction } \\
\hline & & & \multicolumn{2}{|c|}{ TR1 } & \multicolumn{2}{|c|}{ TR2 } \\
\hline & & & CC1 & $\mathrm{CC} 2$ & CC1 & $\mathrm{CC} 2$ \\
\hline \multirow{17}{*}{ 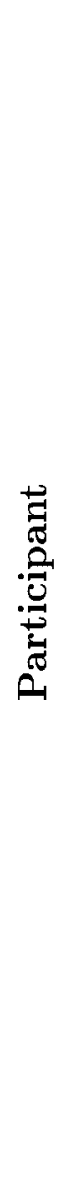 } & E002 & $\begin{array}{l}\text { mTRs } \\
\text { mTRe }\end{array}$ & $\dagger$ & $\dagger$ & $\begin{array}{l}26.66 \\
30.49 \%\end{array}$ & $\begin{array}{c}9.58 \\
11.44 \%\end{array}$ \\
\hline & \multirow{2}{*}{ E003 } & mTRs & 42.68 & 47.56 & $39.00 ※$ & 38.70 \\
\hline & & mTRe & $48.32 ※$ & 52.71 & 32.53 & 35.93 \\
\hline & \multirow{2}{*}{ E004 } & mTRs & 34.84 & 14.20 & 35.74 & 14.04 \\
\hline & & mTRe & $44.06 ※$ & $17.36 \%$ & $38.76 ※$ & $15.70 ※$ \\
\hline & \multirow{2}{*}{ E005 } & mTRs & \multirow{2}{*}{$\dagger$} & \multirow{2}{*}{$\dagger$} & 32.83 & 19.60 \\
\hline & & mTRe & & & 31.49 & 20.73 \\
\hline & \multirow{2}{*}{ E006 } & mTRs & 25.61 & 13.62 & \multirow{2}{*}{$\dagger$} & \multirow{2}{*}{$\dagger$} \\
\hline & & mTRe & $34.25 ※$ & $15.78 ※$ & & \\
\hline & \multirow{2}{*}{ E007 } & mTRs & \multirow{2}{*}{$\dagger$} & \multirow{2}{*}{$\dagger$} & 22.55 & 10.28 \\
\hline & & mTRe & & & $24.99 ※$ & $11.95 ※$ \\
\hline & \multirow{2}{*}{ E008 } & mTRs & 12.28 & 1.90 & 11.26 & 1.34 \\
\hline & & mTRe & 11.25 & 1.82 & 11.36 & 1.27 \\
\hline & \multirow{2}{*}{ E009 } & mTRs & 31.87 & 41.54 & 26.07 & 28.94 \\
\hline & & mTRe & $33.76 ※$ & $46.87 ※$ & $32.21 ※$ & $34.98 \%$ \\
\hline & \multirow{2}{*}{ E010 } & mTRs & 41.72 & 19.98 & 41.39 & 11.71 \\
\hline & & mTRe & 41.21 & 20.20 & 42.58 & 12.32 \\
\hline
\end{tabular}


Table 6.4: Summary of the results from Tables 6.2 and 6.3. The numbers in the columns represent the number of participants, who executed the task correctly, with mTRs $>$ mTRe, $m$ TRs $=$ mTRe and mTRs $<$ mTRe in terms of active muscle stiffness and co-contraction

\begin{tabular}{|c|c|c|c|c|c|c|c|c|c|c|c|c|}
\hline & \multicolumn{6}{|c|}{ TR1 } & \multicolumn{6}{|c|}{ TR2 } \\
\hline & & 邑 & $\stackrel{\mathscr{L}}{\mathrm{Q}}$ & 㽞 & $\bar{U}$ & $\tilde{U}$ & 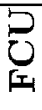 & 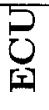 & 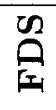 & 导 & $\bar{U}$ & $\underset{\mho}{N}$ \\
\hline mTRs $>$ mTRe & - & 1 & - & - & - & - & - & 2 & - & - & 1 & - \\
\hline $\mathrm{mTRs}=\mathrm{mTRe}$ & 3 & 3 & 4 & 4 & 2 & 3 & 3 & 3 & 5 & 5 & 3 & 4 \\
\hline \multirow[t]{2}{*}{ mTRs $<$ mTRe } & 3 & 2 & 2 & 2 & 4 & 3 & 5 & 3 & 3 & 3 & 4 & 4 \\
\hline & \multicolumn{6}{|c|}{6 participants } & \multicolumn{6}{|c|}{8 participants } \\
\hline
\end{tabular}

\section{Hypothesis TR-HI}

The mean appears to remain high for the duration of both triad exercises. In many cases, mTRs and mTRe are greater than 15\%. E008 is the only participant where mTRs and mTRe are less than $15 \%$ most of the time. Hypotheses TR-HI and SC-LO will be further addressed in Section 8.1.

\section{Hypotheses TR-N1 and TR-N2}

The comments made by the participants after executing TR1 and TR2, visual inspection of the results, and the strategies participants used to carry out these two exercises (i.e. tapping and holding), led to the development of two additional hypotheses.

Hypothesis TR-N1 (triads - new hypothesis \#1): In TR1, there will be a greater proportion of cases where mTRs $<$ mTRe than in TR2.

After having executed TR1 and TR2, many participants commented that the first triad exercise was more challenging (from a co-ordination perspective) and required 
more concentration than the second triad exercise. Given that co-contraction serves as a strategy to improve accuracy and joint stability [28], by extension it is expected that increased co-contraction would be observed under circumstances involving fine movements. If this is true, in the context of these results, it is anticipated that TR1 will have more instances where mTRs $<$ mTRe than TR2; however, this was not the case. There appears to be no large difference between TR1 and TR2.

Hypothesis TR-N2 (triads - new hypothesis \#2): Where tapping was used to play the alternating keys, active muscle stiffness and co-contraction will not change from the start to the end of the exercise, i.e. where a participant tapped the alternating keys mTRs will not be significantly different from mTRe.

Tapping the alternating keys appears to be less challenging than holding the alternating keys. The results presented in Table 6.4 were separated according to which strategy participants used to play the alternating notes of TR1 and TR2. These results are shown in Table 6.5. In TR1, there are no instances where participants who tapped had mTRs $<$ mTRe in terms of active muscle stiffness or co-contraction. More often than not, participants who held the alternating notes had mTRs $<$ mTRe. In TR2, there are no obvious differences between participants who held alternating notes and participants who tapped alternating notes. Overall, there are no large differences due to holding and tapping in TR1 or in TR2; however, the numbers are too small to determine if there are any obvious patterns attributable to holding and tapping. 
Table 6.5: Summary of the results from Tables 6.2 and 6.3, divided according to which strategy (i.e. holding and tapping) the participants used to play the alternating notes in TR1 and TR2. The numbers in the columns represent the number of participants who executed the task correctly with mTRs $>$ mTRe, mTRs $=$ mTRe and $m T R s<m T R e$ in terms of active muscle stiffness and co-contraction.

\begin{tabular}{|c|c|c|c|c|c|c|c|c|c|c|c|c|}
\hline & \multicolumn{6}{|c|}{ TR1 } & \multicolumn{6}{|c|}{ TR2 } \\
\hline HOLDING & & 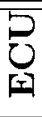 & $\stackrel{\mathscr{O}}{\mathscr{\rho}}$ & $\begin{array}{l}\circlearrowright \\
\text { 宝 }\end{array}$ & $\bar{U}$ & \begin{tabular}{l}
\multirow{U}{U}{} \\
\end{tabular} & $\underbrace{}_{\underbrace{}_{1}}$ & 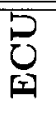 & 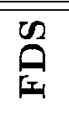 & 吕 & $\overline{0}$ & U \\
\hline mTRs $>$ mTRe & - & - & - & - & - & - & - & 1 & - & - & 1 & - \\
\hline $\mathrm{mTRs}=\mathrm{mTRe}$ & 1 & 2 & 2 & 2 & - & 1 & 2 & 2 & 3 & 3 & 1 & 2 \\
\hline \multirow[t]{2}{*}{ mTRs $<$ mTRe } & 3 & 2 & 2 & 2 & 4 & 3 & 3 & 2 & 2 & 2 & 3 & 3 \\
\hline & \multicolumn{6}{|c|}{4 participants } & \multicolumn{6}{|c|}{5 participants } \\
\hline TAPPING & $\underbrace{}_{\text {它 }}$ & & 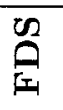 & $\begin{array}{l}\text { 昌 } \\
\text { 星 }\end{array}$ & U & $\begin{array}{l}\mathbb{U} \\
\mathcal{U}\end{array}$ & $\bigodot_{\mathscr{I}}$ & $己_{\text {: }}$ & $\stackrel{\mathscr{2}}{\mathscr{A}_{1}}$ & 吕 & ర & $\begin{array}{l}N \\
\mathcal{U}\end{array}$ \\
\hline mTRs $>$ mTRe & - & 1 & - & - & - & - & - & 1 & - & - & - & - \\
\hline $\mathrm{mTRs}=\mathrm{mTRe}$ & 2 & 1 & 2 & 2 & 2 & 2 & 1 & 1 & 2 & 2 & 2 & 2 \\
\hline \multirow[t]{2}{*}{ mTRs $<$ mTRe } & - & - & - & - & - & - & 2 & 1 & 1 & 1 & 1 & 1 \\
\hline & \multicolumn{6}{|c|}{2 participants } & \multicolumn{6}{|c|}{3 participants } \\
\hline
\end{tabular}




\subsection{Discussion}

\subsubsection{Execution of Triad Exercises}

Although most participants completed the task as required, several lifted their fingers intermittently. Four participants lifted their fingers for a length of time longer than $10 \%$ the length of the exercise, so their results were eliminated. These were E002 (TR1), E005 (TR1), E006 (TR2) and E007 (TR1). In general, participants had more problems with correctly executing TR1 than TR2. This is possibly because of the anatomy of the muscles and tendons controlling the movements of fr 2 , fr3 and fr4: the EDC of the right $\operatorname{arm}^{2}$ has tendons that insert on fr2, fr3, fr4 and fr5. These tendons are branches from a single tendon that comes off the EDC; fr2 and fr5 are each controlled by an additional muscle, the EIP and EDM, respectively, that is specific to that digit only. TR1 required that fr1, fr3 and fr5 hold down piano keys, while fr2 and fr4 alternately moved to depress E4 and G4 keys; TR2 required that fr1, fr2 and fr5 hold down piano keys, while fr3 and fr4 alternately moved to depress F4 and G4 keys. It is possible that TR2 relied on greater contributions from the EIP and EDM than TR1 to keep fr2 and fr5 depressed, which might possibly explain why more participants successfully executed TR2 than TR1.

Unexpectedly, some of the participants tapped the alternating notes, rather than playing them in a legato manner. The researcher demonstrated the task to the participants; they received no instruction that they were to play the alternating keys in a legato manner. It is assumed that some participants tried to overcome the difficulty of the task by tapping the alternating notes, such as E005 (TR1 and TR2), E008 (TR1), E009 (TR2) and E010 (TR1 and TR2), while others played the alternating

\footnotetext{
${ }^{2}$ The EDC of the left arm is no different than the EDC of the right arm. We make the distinction between right and left here because the " $\mathrm{r}$ " in "fr" refers to the right hand.
} 
notes in a legato manner yet struggled to get through the exercise, such as E002 (TR1).

The results of participant E009 were atypical: this participant held the alternating notes in TR1, but tapped the alternating notes in TR2. This observation was unexpected because of the two triad exercises, TR1 is more challenging. TR 1 presents an anatomical challenge that TR2 does not. Thus, it was expected that participants who were able to hold the alternating keys in TR1 would not tap them in TR2.

\subsubsection{EMG Results and Hypothesis TR-SC}

Initially it was hypothesized that there would be a significant increase in active muscle stiffness and co-contraction from the start of the exercise to the end of the exercise. To measure if this change took place, the means of segments 2 and 4 were compared. The number of participants that experienced significant increases in the mean active muscle stiffness and co-contraction and the number of participants who experienced no significant change in these measurements were approximately equal. There were slightly more participants with no significant changes in mean active muscle stiffness, and slightly more participants with significant increases in mean co-contraction.

Closer examination of Tables 6.2 and 6.3 shows that significant increases in active muscle stiffness and co-contraction appear to be participant-related, rather than taskrelated. For instance, participants E008 and E010 experienced very few significant changes in active muscle stiffness and co-contraction in TR1 and TR2; participants E004 and E009 experienced significant increases in CC1 and CC2 in both TR1 and TR2. It is difficult to say if all results are participant-related, because a number of results have been eliminated from the analysis due to improper task execution. Also, the results of participant E003 did not follow this pattern: during TR1, active 
muscle stiffness of the FCU and CC1 increased significantly, but in TR2 active muscle stiffness of the ECU and CC1 decreased significantly.

As noted in Section 6.1.1, most participants stated that they found TR1 more challenging than TR2 (i.e. participants felt stiffer and experienced more discomfort when executing TR1 than TR2); however, this is not reflected in the results. If TR1 was more difficult, the expectation is that there would be more significant increases in active muscle stiffness and co-contraction in TR1 than in TR2, which is proposed in hypothesis TR-N1; however, this was not the case. It is possible that because TR1 results of three participants and TR2 results of one participant were eliminated on account of these participants not properly executing the task, the results were skewed.

To verify if TR1 caused higher levels of active muscle stiffness and co-contraction than TR2, their averages could be compared. This analysis was not done here because participants held the alternating notes for varying lengths of time, and currently it is unclear what effect tapping, instead of holding, the alternating notes has. If this study were to be repeated, participants should be instructed to play the alternating notes in a legato manner, and to play alternating notes to the beat of a metronome.

As identified in hypothesis TR-N2, it is suspected that tapping the alternating notes in the finger independence exercises is less challenging than holding them. Participants may have used tapping as a strategy to overcome difficulties they faced in executing TR1 and TR2. This study neither confirmed nor rejected hypothesis TRN2. To test hypothesis TR-N2 further, a new study should be carried out. Under this new study, participants would be asked to perform TR1 and TR2 first by holding the alternating notes, then by tapping the alternating notes. Mean values of active muscle stiffness and co-contraction would be calculated for the full length of each exercise. For each participant in the new study comparisons of all four measures of active muscle stiffness and both measures of co-contraction would be made for the 
two strategies (i.e. holding and tapping the alternating notes) used to execute TR1 and TR2. In addition, the results of the new study should be analyzed using the same methods employed in the current study, i.e. mean active muscle stiffness and co-contraction of segments 2 and 4 should be compared. Determination of whether significant changes in active muscle stiffness and co-contraction were more commonly observed using the tapping strategy or the holding strategy would be possible.

Another way to improve our study, in addition to the hypothetical study described in the previous paragraph, would be to measure the MES from more than four muscles. The participants noted an increase in discomfort during the task, but a significant increase in active muscle stiffness and co-contraction was observed approximately $50 \%$ of the time. The discomfort that occurs might be due to an increase in active muscle stiffness and co-contraction of other muscles that were not measured, such as the FDP, ECRL and ECRB. The actions of the EDC might be offset not only by the FDS but also by the FDP. The hypothesized additional contraction in the FDP might require some additional stabilization, hence co-contraction, at the wrist.

\subsubsection{EMG Results and Hypothesis TR-HI}

Except in the case of the results of participant E008, the levels of mean active muscle stiffness and co-contraction were greater than $15 \% \mathrm{MVC}$, meaning they are not low. In the literature, there does not seem to be a clear definition of what qualifies as high active muscle stiffness and co-contraction. As already mentioned in Section 5.2.2, the mean values of active muscle stiffness and co-contraction might be as high as they are due to the normalization methods used. On several plots, the linear envelope of mean active stiffness or co-contraction exceeds $100 \%$, which does not make sense from a physiological standpoint, assuming the MVC is the greatest force a muscle can generate. Normalization techniques will be discussed in Chapter 8 . 
If active muscle stiffness and co-contraction were already high at the start of the exercise, it is possible that they did not increase because one or several muscles had already reached the maximum force it can generate in that anatomical configuration. Furthermore, active muscle stiffness and co-contraction may not have increased due to the effects of reciprocal inhibition. Reciprocal inhibition is the inhibition of antagonist muscle contraction during the contraction of an agonist muscle. It is regulated by a neural mechanism [57].

\subsection{Outcomes of Hypothesis Testing}

In this chapter, four hypotheses were proposed:

1. Hypothesis TR-SC: Active muscle stiffness and co-contraction will significantly increase from the start to the end of TR1 and TR2.

This hypothesis was confirmed, but requires further investigation as participants in this study used two distinct strategies (i.e. holding and tapping the alternating notes of TR1 and TR2) to execute the exercise.

2. Hypothesis TR-HI: Active muscle stiffness and co-contraction will be high throughout the duration of the exercise.

This hypothesis was confirmed.

3. Hypothesis TR-N1: Significant increases in active muscle stiffness and cocontraction will be observed more frequently in TR1 than in TR2.

This hypothesis was rejected, although this may attributed to the elimination of results of participants who did not execute the task as required. 
4. Hypothesis TR-N2: Significant increases in active muscle stiffness and cocontraction will not be observed where participants tapped the alternating notes in TR1 and TR2.

This hypothesis was rejected, but requires further investigation as there were not enough participants to establish any discernible patterns. 


\section{Chapter 7}

\section{Task \#3: Results and Discussion}

The composition by Ann Southam is a piece of music that is 26 bars in length and takes 0:02:36 to play when executed correctly. The musical score is presented in Figure 7.1.

\subsection{Results of Task \#3: Piece by Ann Southam}

The Ann Southam piece was composed to induce an increase in wrist stiffness, even in experienced pianists, after being played for a minute [3]. As the piece progresses, its tempo increases (meaning its note rate increases) as do the dynamic levels (meaning it gets louder), both of which are hypothesized to increase muscle stiffness. Sections of the piece are repeated to allow for comparison. The note pattern of the piece is repeated over and over with slight modifications to the pattern so that it is largely unpredictable, forcing the participant to read the music, rather than "play by ear" or from memory. The piece requires minimal lateral movement of the arm, as each finger always plays the same note.

The participants were asked to play the piece twice. Initially, they were given several minutes to look over the music, but were not allowed to play. During this 

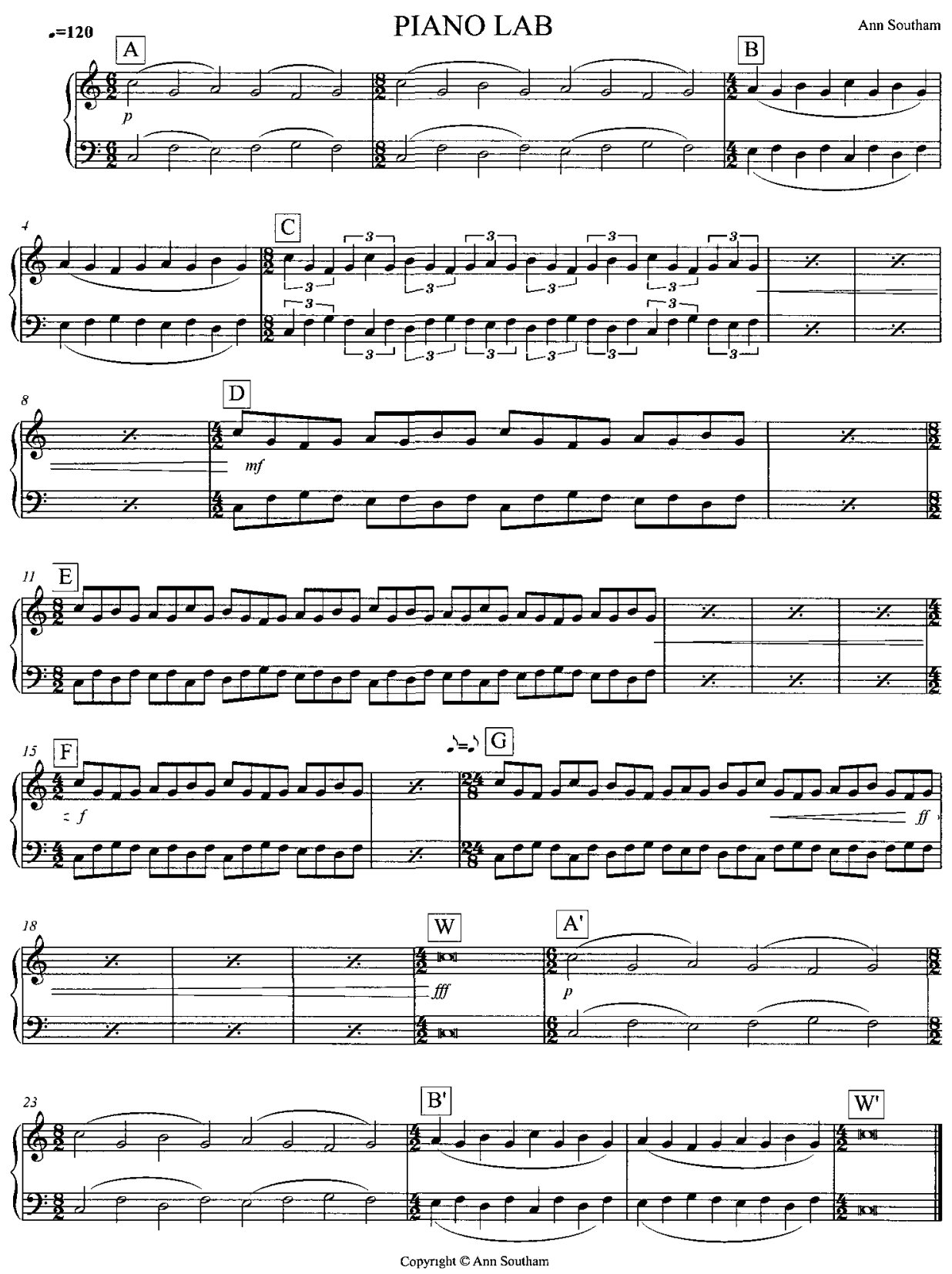

Figure 7.1: Musical score of piece composed by Ann Southam. Each letter marks a different section of the piece. 
time several hints to help them correctly perform the piece were offered. They were told that each finger always played the same note, meaning that their hands did not need to move up and down the keyboard. Participants were also told that the right and left hands mirror each other in their playing pattern (e.g. when the right thumb plays F4 or midi note 65, the left thumb should be playing G3 or midi note 55), and that every other note is G4 or midi note 67 . The musical score was reviewed with each participant to ensure they understood the notation and the dynamics. This was done to minimize the number of errors played by the participant and to reduce the variability of the performance of the piece between participants. Participants were given the choice to play with a metronome, but only E003 chose to.

\subsubsection{Execution of the Piece by Ann Southam}

Overall, the participants were successful in executing the piece; however, no one played the piece perfectly. The performance of the piece was assessed for its note accuracy and for the participants' observation of musical dynamics and tempo. These measurements of performance are described in the following three sections. Participants were not given the opportunity to prepare the piece in advance as practice might lead to decreased levels of active muscle stiffness and co-contraction. In a study by Gribble et al. [27] participants performed pointing movements trials; they found that co-contraction and associated limb stiffness decreased over the course of these trials.

\section{Note Accuracy}

Note accuracy indicates how many deviations the participants made from the correct note order of the piece. The note duration and the time elapsed between note off and note on of the subsequent piano keys were not considered in reporting note accuracy. (Anomalies in note duration and time elapsed between notes are captured 
in the tempo analysis.) Although the MIDI data includes notes played by the left hand and the right hand, only the note accuracy of the right hand was considered, as only EMG data were collected from the muscles of the right forearm.

To understand where participants made the most errors, the piece was divided into three sections: beginning, middle and end. The "beginning" is made up of sections A, B and C; the "middle" is made up of sections D, E, F and G; the "end" is made up of sections W, A', B' and W'. (Refer to Figure. 7.1 to see where these sections fall in the piece.) These divisions were chosen based on note duration. If the time signatures are correctly followed in bars 9-20, every note has the same duration $(0.25 \mathrm{~s})$. The beginning and end sections (excluding bars 21 and 26) have longer note durations, ranging from $0.33 \mathrm{~s}$ to $1.0 \mathrm{~s}$. Bars 21 and 26 are whole notes, with durations of $4.0 \mathrm{~s}$.

Three types of note accuracy errors were considered: additions (added notes), deletions (missed notes) and substitutions (incorrect notes). The three types of note accuracy errors were counted in all three sections for both times the participants played the piece.

The "diff" algorithm in Linux was used to detect errors, although ultimately all errors were counted manually. In cases where there were several consecutive errors or errors within close proximity, often there were multiple ways to interpret these errors. Depending on how the errors were interpreted, this yielded different numbers of note accuracy errors. The analysis that yielded the fewest total note accuracy errors was used. For example, when participant E004 played the Ann Southam piece for the first time, in one of the repetitions of bar 17 the participant played 67-69-67-69-71. The correct note sequence is 67-65-67-69-67-71. These errors can be interpreted in at least two ways:

1. 67 - deletion - deletion - 69 - 67- [addition of 69] - 71, or 
2. 67 - substitution - $67-69$ - deletion - 71 .

The first method of counting errors yields one addition error and two deletion errors, for a total of three note accuracy errors. The second method of counting errors yields one deletion error and one substitution error, for a total of two note accuracy errors. This error was scored as one deletion error and one substitution error.

Tables 7.1 and 7.2 show that participants made the fewest number of errors in the beginning and end sections, and made the most errors in the middle section. This was expected as the middle section has a higher note rate and contains more notes: the middle section is composed of 288 notes, while the beginning and end sections are composed of 126 and 32 notes, respectively.

Table 7.1: Tally of participants' addition (add), deletion (del) and substitution (sub) note accuracy errors made in the beginning, middle and end sections of the first attempt of the Ann Southam piece.

\begin{tabular}{|c|c|c|c|c|c|c|c|c|c|c|c|c|c|}
\hline \multirow{3}{*}{\multicolumn{2}{|c|}{$\begin{array}{r}\text { Location } \\
\text { Error type }\end{array}$}} & \multicolumn{12}{|c|}{ Attempt 1} \\
\hline & & \multicolumn{3}{|c|}{ Beginning } & \multicolumn{3}{|c|}{ Middle } & \multicolumn{3}{|c|}{ End } & \multicolumn{3}{|c|}{ total } \\
\hline & & add & del & sub & add & del & sub & add & del & sub & add & del & sub \\
\hline \multirow{9}{*}{ } & $\mathrm{E} 002$ & 1 & - & - & 3 & 2 & 7 & - & 2 & - & 4 & 4 & 7 \\
\hline & E003 & 6 & - & - & 21 & - & 2 & - & - & - & 27 & - & 2 \\
\hline & E004 & - & - & - & 21 & 8 & 4 & - & - & - & 21 & 8 & 4 \\
\hline & E005 & - & - & - & 2 & - & 4 & - & - & - & 2 & - & 4 \\
\hline & E006 & 1 & 1 & 2 & 58 & 1 & 1 & - & - & - & 59 & 2 & 3 \\
\hline & E007 & - & 6 & 3 & 25 & 12 & 5 & - & - & - & 25 & 18 & 8 \\
\hline & E008 & 15 & 18 & 4 & 44 & 9 & 11 & - & - & - & 59 & 27 & 15 \\
\hline & E009 & 13 & - & - & 29 & - & 1 & 1 & - & - & 43 & - & 1 \\
\hline & E010 & 1 & - & 1 & 6 & 2 & - & - & - & - & 7 & 2 & 1 \\
\hline
\end{tabular}

A large number of the errors that were observed in the middle section were addition errors, e.g. E003 attempt 1, E006 attempts 1 and 2, and E008 attempt 1. In some 
Table 7.2: Tally of participants' addition (add), deletion (del) and substitution (sub) note accuracy errors made in the beginning, middle and end sections of the second attempt of the Ann Southam piece.

\begin{tabular}{|c|c|c|c|c|c|c|c|c|c|c|c|c|c|}
\hline \multirow{3}{*}{\multicolumn{2}{|c|}{\begin{tabular}{|l|} 
Location \\
Error type
\end{tabular}}} & \multicolumn{12}{|c|}{ Attempt 2} \\
\hline & & \multicolumn{3}{|c|}{ Beginning } & \multicolumn{3}{|c|}{ Middle } & \multicolumn{3}{|c|}{ End } & \multicolumn{3}{|c|}{ total } \\
\hline & & add & del & sub & add & del & sub & add & del & sub & add & del & sub \\
\hline \multirow{9}{*}{ 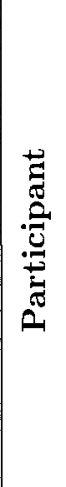 } & E002 & 2 & - & 2 & 8 & 9 & 11 & - & 2 & - & 10 & 11 & 13 \\
\hline & E003 & - & - & - & - & - & - & - & - & - & - & - & - \\
\hline & E004 & - & - & - & 10 & 9 & 4 & - & - & 1 & 10 & 9 & 5 \\
\hline & E005 & - & - & - & - & - & - & - & - & - & - & - & - \\
\hline & E006 & - & - & 1 & 25 & 5 & 8 & - & - & - & 25 & 5 & 9 \\
\hline & E007 & 1 & - & - & 30 & 1 & 2 & - & - & - & 31 & 1 & 2 \\
\hline & E008 & - & - & 2 & 6 & 4 & 24 & - & - & - & 6 & 4 & 26 \\
\hline & E009 & 4 & - & - & 8 & - & - & - & - & - & 12 & - & - \\
\hline & E010 & 1 & - & - & 2 & - & - & - & - & - & 3 & - & - \\
\hline
\end{tabular}

cases, the large number of addition errors was due to participants "self-correcting" or restarting at the beginning of a bar after having played several notes. In the second attempt of the piece participant E008 - and to a lesser extent, participant E002 in her first attempt of the piece - made more substitution errors than addition or deletion errors. Participants E002 and E008 were two of four undergraduate music performance students who took part in the study. Music performance majors are taught to proceed to the next note in the piece should they make a mistake. This may explain why participants E002 and E008 made more substitution errors than addition errors.

In addition to note accuracy errors, participants also made repetition or omission errors. A repetition error is similar to an addition error, except that an entire bar is repeated (with or without note accuracy errors) more times than is indicated in the piece. An omission error is similar to a deletion error, except that it occurs where a 
participant leaves out an entire bar. If repetition and omission errors were not counted separately, and were counted as note addition and deletion errors instead, this would overestimate note accuracy errors, when the problem is that the participant lost track of how many times he or she had repeated a section. For example, participant E005 made no note accuracy errors when playing the piece for a second time (see Table 7.2), but repeated bar $20^{1}$ an extra time (see Table 7.3). (Bar 20 falls in the middle section.) By counting E005's one repetition error as 24 addition errors, the note accuracy results of E005 would not be distinguishable from those of a hypothetical participant who made 24 addition errors throughout the piece. From a musician's perspective, it is clear that E005 played the piece more accurately than the hypothetical participant; however, our accuracy analysis would not reflect this. For this reason, repetition and omission errors are quantified separately from accuracy errors.

Participants made repetition and omission errors in sections C, D, E, F and G, and these errors are tallied in Table 7.3; sections A (bars 1 and 2), B (bars 3 and 4), W (bar 21), A' (bars 22 and 23), B' (bars 24 and 25) and W' (bar 26) were not included in Table 7.3 as participants made no repetition or omission errors in these sections. The majority of participants made repetition or omission errors in section $G$ (see Table 7.3). In attempt 1 of the piece, a total of 14 repetition or omission errors were made in section $G$ when the results of all participants were pooled. The same number of repetition or omission errors were made in the second attempt of the piece.

Generally speaking, the second attempt of the piece was played with fewer errors: participants made fewer note accuracy errors and fewer sections of the piece were repeated or omitted the second time the piece was played.

\footnotetext{
${ }^{1}$ It is impossible to know whether the participant repeated bar 17, 18, 19 or 20 since the symbol in bars 18,19 and 20 indicates to repeat bar 17
} 
Table 7.3: Bars repeated or omitted by all participants during the first and second attempts of the Ann Southam piece. Positive integers indicate how many extra times a bar was repeated; negative integers indicate how many times a bar was omitted. Bars that have the same note sequence (e.g. bars 5, 6, 7 and 8) are combined into one entry in the table.

\begin{tabular}{|c|c|c|c|c|c|c|c|}
\hline & Section & $\mathrm{C}$ & $\mathrm{D}$ & $\mathbf{E}$ & $\mathrm{F}$ & G \\
\hline & & \multirow{4}{*}{ Bar } & 5 & 9 & 11 & 15 & 17 \\
\hline & & & 6 & 10 & 12 & 16 & 18 \\
\hline & & & 7 & & 13 & & 19 \\
\hline & & & 8 & & 14 & & 20 \\
\hline \multirow{18}{*}{ 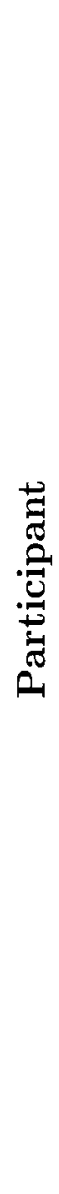 } & \multirow{9}{*}{ 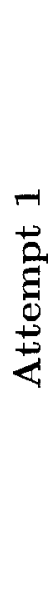 } & E002 & 1 & & 2 & & \\
\hline & & E003 & & & & 2 & 2 \\
\hline & & E004 & & & -1 & & 3 \\
\hline & & E005 & & & & & 1 \\
\hline & & E006 & & 1 & & & 1 \\
\hline & & E007 & 1 & 1 & & & 4 \\
\hline & & E008 & & & & & \\
\hline & & E009 & & & & & 2 \\
\hline & & E010 & -1 & & -1 & & 1 \\
\hline & \multirow{9}{*}{ 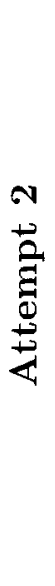 } & E002 & 2 & & & & 3 \\
\hline & & E003 & & & & & 2 \\
\hline & & E004 & & & 1 & & 2 \\
\hline & & E005 & & & & & 1 \\
\hline & & E006 & 1 & & & -1 & 1 \\
\hline & & E007 & & & 1 & & 1 \\
\hline & & E008 & & & & & 3 \\
\hline & & E009 & & & & & \\
\hline & & E010 & & -1 & -1 & & 1 \\
\hline
\end{tabular}


Sections C, D, E, F and G vary in the number of notes they contain from participant to participant because of the note accuracy, repetition and omission errors each made. For that reason, section $\mathrm{C}$ was redefined as the first 96 notes following the end of section B, and was renamed C96. Similarly, section G was redefined as the 96 notes preceding section $\mathrm{W}$, and was renamed section G96. There are two exceptions, which are:

- Participant E008 - attempt 1 - section $\mathrm{C}=90$ notes

- Participant E010 - attempt 1 - section $\mathrm{C}=73$ notes

Both made deletion or omission errors in section $C$, and thus there are fewer than 96 notes in this section for them. Section $\mathrm{C}$ was redefined according to the number of notes participants played in this section. Participant E008's section C was renamed C90; participant E010's section C was renamed C73. Sections D, E and F were not considered in all other analyses. These sections have the same note rate as section G96, but the most significant changes in active muscle stiffness and co-contraction are expected to have taken place in section G96 where the piece is meant to be played at its loudest. The changing levels of loudness are evaluated in the following section.

\section{Dynamics}

In the musical sense, the dynamics of a piece of piano music refer to the loudness of its notes. The amplitude of the sound can be measured; however, it should be noted that dynamic markings are relative to the piece as a whole. They are subject to interpretation by the performer and the amplitude of the sound is influenced by the abilities of the performer. That said, the music software company, Tanager AudioWorks Inc., assigns key velocity ranges to various dynamic levels [58], as outlined in Table 7.4. In the Ann Southam piece the piece starts quietly (or $p$ ), and there is 
a. gradual crescendo (or an increase in loudness) until the end of bar 21 where the dynamics indicate to play very loudly (or $f f f$ ).

Table 7.4: MIDI key velocity ranges and associated dynamic levels (adapted from Chirp Virtual MIDI Keyboard Controller User Guide - Build 1.2) [58].

\begin{tabular}{|c|l|}
\hline Velocity range & Music terminology term (and abbreviation) \\
\hline $1-15$ & Extremely soft or pianississimo $(p p p)$ \\
\hline $16-30$ & Pianissimo $(p p)$ \\
\hline $31-45$ & Piano $(p)$ \\
\hline $46-60$ & Mezzo piano $(m p)$ \\
\hline $61-75$ & Mezzo forte $(m f)$ \\
\hline $76-90$ & Forte $(f)$ \\
\hline $91-105$ & Fortissimo $(f f)$ \\
\hline $102-127$ & Extremely loud or fortisissimo $(f f f)$ \\
\hline
\end{tabular}

On the Disklavier piano, note loudness can be determined by measuring key velocity. The Disklavier piano possesses continuous-position optical sensors for measuring key velocity. It is a value from 0 to 127 and has no units; the higher the key velocity, the louder the note. The software used to generate the score of the Ann Southam piece also produced a MIDI file with key velocities for each note matching the dynamics of the piece. This MIDI file was treated as the ideal way to play the piece. The Tukey-Kramer test (with the alpha level of probability set at 0.05 ) was used to compare the mean key velocities of the ideal case. This test revealed that:

- the mean key velocity was higher in section B than in section A, but not significantly;

- the mean key velocity was higher in section C96 than in sections A and B, but not significantly; 
- the mean key velocity was significantly higher in section G96 than in sections A, B and C96;

- the mean key velocity was significantly higher in section $W$ than in sections $A$, B, C96 and G96.

To determine if participants observed the dynamics of the piece, the mean key velocities were found for sections A, B, C96, G96, W, A', B' and W', and the TukeyKramer test (with the alpha level of probability set at 0.05) was used to establish which means were significantly different.

Participant E003's mean key velocity results from the first attempt of the piece, depicted in Figure. 7.2(a), were consistent with the ideal case. Figure. 7.2(a) shows that:

- the mean key velocity in section $B$ was higher than in section A, but not significantly;

- the mean key velocity in section C96 was higher than in section A and lower than in section $\mathrm{B}$, but not significantly in either case;

- the mean key velocity in section G96 was significantly higher than in sections A, B and C96;

- the mean key velocity in section $\mathrm{W}$ was significantly higher than in sections $\mathrm{A}$, $\mathrm{B}$ and $\mathrm{C} 96$; however, the mean key velocity in section $\mathrm{W}$ was higher than in section G96, but not significantly.

Figure. 7.2(b) shows that participant E003's mean key velocity results from the second attempt of the piece are similar to the results from the first attempt. The 


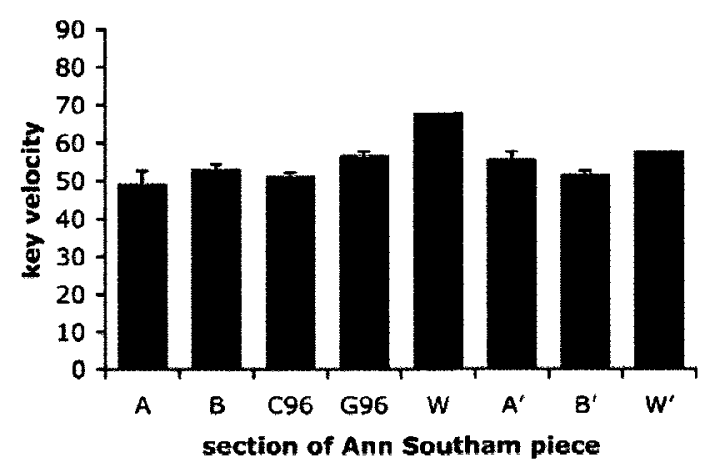

(a) Attempt 1

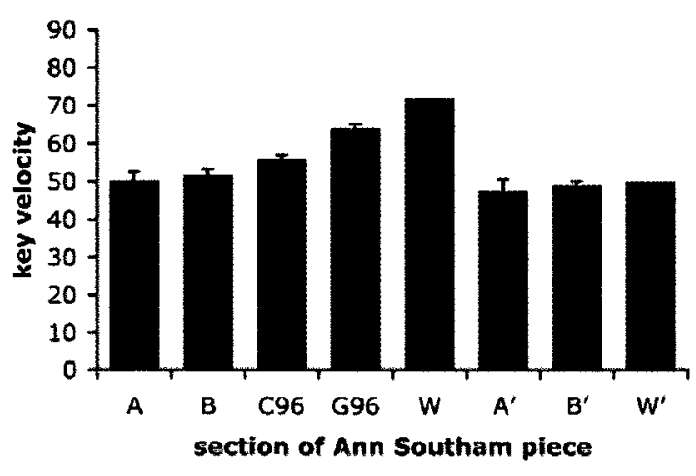

(b) Attempt 2

Figure 7.2: Mean key velocities of sections A, B, C96, G96, W, A', B' and W' in Ann Southam piece for participant E003. Mean key velocities are shown for (a) attempt 1 - blue, and (b) attempt 2 - red. Error bars represent $95 \%$ confidence intervals. There are no error bars for $W$ and $W$ ' because these sections are each made up of only one note.

only difference is that mean key velocity was significantly higher in section C96 than in sections $\mathrm{A}$ and $\mathrm{B}$.

Not all participants' results were consistent with this pattern. For example, in participant E006's first attempt of the piece, the mean key velocity was highest in section $\mathrm{W}$, then $\mathrm{A}$, then $\mathrm{C} 96$, then $\mathrm{B}$, then G96. These results are shown in Figure. 7.3(a). The differences in mean key velocity were not significant. In participant E006's second attempt of the piece, the mean key velocity was highest in section G96, then C96, then $W$, then B, then A. These results are shown in Figure. 7.3(b). This is more consistent with the dynamics in the ideal case; however, the results were not significant.

Appendix $\mathrm{N}$ contains figures showing the mean key velocities, with error bars representing $95 \%$ confidence intervals, for all participants in this study. 


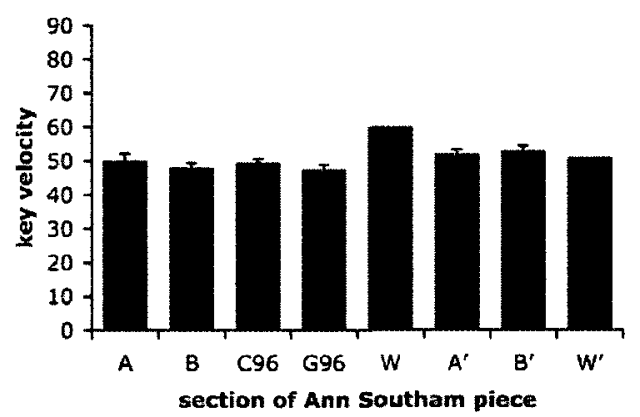

(a) Attempt 1

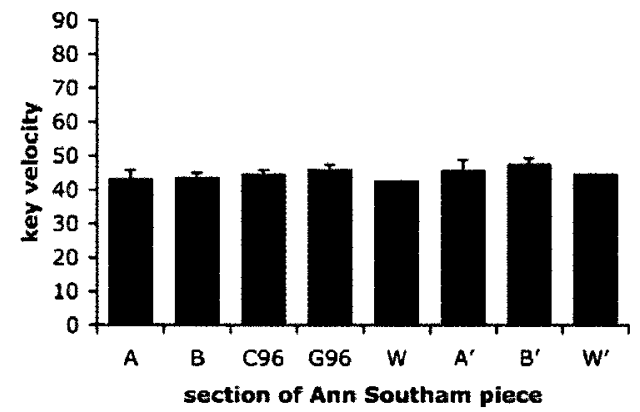

(b) Attempt 2

Figure 7.3: Mean key velocities of sections A, B, C96, G96, W, A', B' and 'W' in Ann Southam piece for participant E006. Mean key velocities are shown for (a) attempt 1 - blue, and (b) attempt 2 - red. Error bars represent $95 \%$ confidence intervals. There are no error bars for $W$ and $W$ ' because these sections are each made up of only one note.

\section{Tempo}

The tempo of the piece was set to $120 \mathrm{bpm}$ using a metronome; however, participants were allowed to play without it if they chose. Participant E003 was the only one who elected to use a metronome (although set the metronome to flash, rather than to beat). As most participants chose not to use the metronome, there was a tendency to start too slowly and to slow down as the participant progressed through the piece. The tempo was reported as the mean number of notes played per second by the right hand (i.e. as the note rate), as it was in Chapter 5 .

The mean note rates in each section in both attempts of the piece are presented in Table 7.5. Table 7.5 also lists the ideal note rate - the number of notes per second that should have been played in that section had the indicated tempo and time signature been followed. When a participant's note rate was higher than the ideal case, the participant was playing faster than the indicated tempo; when a participant's note rate was lower than the ideal case, the participant was playing slower than the 
Table 7.5: Participants' mean note rates in sections A, B, C96, G96, W, A', B' and W' of the Ann Southam piece, expressed in notes per second. Results of both attempts, as well as the ideal case, are shown. When the piece is played at the indicated tempo, and all time signatures are followed, the note rate is the same as it is during the ideal case. A participant played slower than the indicated tempo when his or her note rate is lower than the ideal case; a participant played faster than the indicated tempo when his or her note rate is higher than the ideal case. Only participant E003 chose to play with a metronome.

\begin{tabular}{|c|c|c|c|c|c|c|c|c|c|c|}
\hline \multirow{2}{*}{\multicolumn{3}{|c|}{ section }} & \multicolumn{8}{|c|}{ Note rate (in notes/s) } \\
\hline & & & A & B & C96 & G96 & W & $\mathbf{A}^{\prime}$ & $\mathrm{B}^{\prime}$ & $\mathbf{W}^{\prime}$ \\
\hline \multicolumn{3}{|r|}{ ideal } & 1.00 & 2.00 & 3.00 & 4.00 & 0.25 & 1.00 & 2.00 & 0.25 \\
\hline \multirow{18}{*}{ 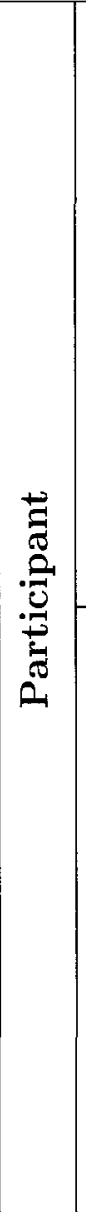 } & \multirow{18}{*}{ 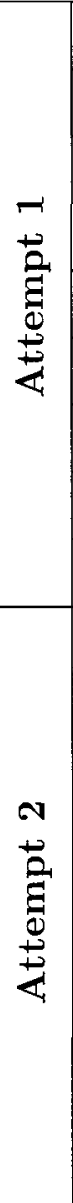 } & E002 & 0.83 & 1.56 & 2.77 & 4.24 & 0.34 & 0.75 & 1.90 & 0.27 \\
\hline & & E003 & 0.93 & 1.91 & 2.18 & 4.85 & 0.17 & 0.69 & 1.62 & 0.22 \\
\hline & & E004 & 1.13 & 2.28 & 3.52 & 3.69 & 0.26 & 1.00 & 2.38 & 0.26 \\
\hline & & E005 & 1.23 & 2.68 & 3.91 & 3.70 & 0.26 & 0.96 & 2.88 & 0.20 \\
\hline & & E006 & 1.08 & 2.11 & 3.79 & 3.63 & 0.29 & 1.00 & 2.11 & 0.17 \\
\hline & & E007 & 0.80 & 1.53 & 3.11 & 4.42 & 0.37 & 0.72 & 1.61 & 0.21 \\
\hline & & E008 & 0.95 & 2.09 & 3.54 & 4.41 & 0.25 & 0.84 & 2.15 & 0.23 \\
\hline & & $\mathbf{E}$ & 0.57 & 1.33 & 3.08 & 3.81 & 0.14 & 0.68 & 1.74 & 0.19 \\
\hline & & E010 & 1.01 & 2.14 & 2.84 & 3.90 & 0.51 & 1.21 & 2.56 & 0.34 \\
\hline & & E002 & 0.81 & 1.65 & 2.91 & 4.13 & 0.34 & 0.73 & 1.74 & 0.26 \\
\hline & & E003 & 0.78 & 1.72 & 2.41 & 4.78 & 0.27 & 0.77 & 1.76 & 0.24 \\
\hline & & E004 & 0.94 & 1.96 & 3.50 & 3.60 & 0.34 & 0.84 & 2.23 & 0.34 \\
\hline & & E005 & 1.11 & 2.84 & 4.21 & 4.18 & 0.24 & 0.96 & 2.61 & 0.17 \\
\hline & & E006 & 0.99 & 2.00 & 3.60 & 3.37 & 0.27 & 0.90 & 1.82 & 0.28 \\
\hline & & E007 & 0.74 & 1.60 & 3.35 & 4.42 & 0.26 & 0.73 & 1.66 & 0.22 \\
\hline & & E008 & 0.89 & 2.00 & 3.67 & 4.90 & 0.18 & 0.80 & 1.98 & 0.31 \\
\hline & & E009 & 0.63 & 1.47 & 3.26 & 4.79 & 0.26 & 0.69 & 1.72 & 0.32 \\
\hline & & E010 & 1.18 & 2.52 & 3.19 & 4.42 & 0.43 & 1.16 & 2.44 & 0.30 \\
\hline
\end{tabular}


indicated tempo.

In the ideal case, the note rate in section $A$ is 1 note/s. When section $B$ starts, the note rate increases to 2 notes/s. At the beginning of section C96 the note rate increases to 3 notes/s. The highest note rate is in section G96 where it is 4 notes/s; the lowest note rate is in sections $W$ and $W^{\prime}$, where it is 0.25 notes/s (which can also be described as $4 \mathrm{~s}$ to play one note). The note rates in sections $\mathrm{A}$ and $\mathrm{A}^{\prime}$ are identical, as they are in sections B and B' and sections $\mathrm{W}$ and $\mathrm{W}^{\prime}$.

Initially, participant E006 played the piece at tempo (see Table 7.5, E006 attempts 1 and 2, sections A and B), played faster than tempo in section C96, but slowed down in section G96. Participant E003 did the opposite in the second attempt of the piece. Initially, participant E003 played slower than the indicated tempo, continued to play slower than $120 \mathrm{bpm}$ in sections B and C96, then sped up and played faster than 120 bpm in section G96. Different still, in both attempts of the piece participant E007 played slower than $120 \mathrm{bpm}$ in sections $\mathrm{A}$ and B, then sped up in section C96 and continued to play faster than the set tempo in G96.

According to Table 7.5, in both attempts of the piece, participants E005 and E006 had a higher note rate in section C96 than in G96. Conversely, participants E002 and E003 played slower than the set tempo in section C96, but sped up and played considerably faster than the set tempo in section G96. This occurred in both of their attempts of the piece.

For the most part, participants played slower in section A' than they did in section A, indicated by a smaller note rate in A' than in A in Table 7.5. Participants E009 and E010 are exceptions to this generalization. They both played at a faster tempo in A' than in A during their first attempt, but only E009 played at a faster tempo in A' than in A during the second attempt of the piece. 


\subsubsection{Hypotheses - Piece by Ann Southam}

As already mentioned at the beginning of Section 7.1, the piece composed by Ann Southam was written so that it would induce an increase in wrist stiffness, even in experienced pianists, after being played for a minute. The piece increases in note rate from bars 1-20 (and returns to lower note rates in bars 21-26). It also increases in loudness from bars 1-21 (and returns to lower loudness levels in bars 22-26). As such, two hypotheses are proposed:

Hypothesis AS-SC (Ann Southam - significant change): There will be a significant change in levels of active muscle stiffness and co-contraction from the beginning of the piece to the end of bar 20 .

Hypothesis AS-BE (Ann Southam - beginning versus end): Following the middle passage, the first four bars of the piece are repeated at the end of the piece. In the middle passage, the note rate is higher and the notes are played more loudly. The levels of active muscles stiffness and co-contraction at the beginning and end of the piece will be significantly different.

a. Active muscle stiffness and co-contraction will be higher in $A^{\prime}$ than in A, because A' follows almost immediately after the middle passage.

b. Active muscles stiffness and co-contraction will be higher in B' than in B because $\mathrm{B}^{\prime}$ comes after the middle passage.

c. Active muscle stiffness and co-contraction will be higher in W than in W' because $W$ comes directly after the middle passage, and $W^{\prime}$ comes after sections A' and B', which do not have as high a note rate as the middle passage, nor are these sections as loud as the middle passage. 
To test hypothesis AS-SC, the mean EMG levels for sections A, B, C96 and G96 will be compared to one another (using paired comparisons) to see if there is a significant change in active muscle stiffness and co-contraction throughout the piece.

To test hypothesis AS-BE, paired comparisons of mean EMG levels will be made for sections $A$ and $A^{\prime}, B$ and $B^{\prime}$, and $W$ and $W^{\prime}$.

\subsubsection{EMG results of the piece by Ann Southam}

EMG files were processed and trimmed as described in Chapter 4. EMG data for each of the four muscles measured were plotted versus time, and co-contraction data (CC1 and CC2) were plotted versus time. As an example, the results of participant E002's first attempt of the Ann Southam piece are shown in Figure. 7.4.

\section{Hypothesis AS-SC}

Participant E002's results are typical of how most participants performed. Plots of all participants' active muscle stiffness and co-contraction results can be found in Appendix $\mathrm{O}$ and Appendix $\mathrm{P}$, respectively. E002's results show that initially active muscle stiffness and co-contraction were low, but as E002 progressed through the piece, active muscle stiffness and co-contraction increased. Visually, it appears that mean active muscle stiffness and co-contraction increased from $\mathrm{A}$ to $\mathrm{B}$, from $\mathrm{B}$ to $\mathrm{C} 96$, from C96 to G96, decreased from G96 to W and from W to A', increased from A' to B', then decreased from B' to W'. This was confirmed by ANOVA. The EMG files were split into eight sections, as described in Chapter 4, corresponding to the equivalent divisions in the MIDI files: A, B, C96, G96, W, A', B' and W'. (As already mentioned in Section 7.1.1, sections D, E and F were left out of the analysis because they have the same note rate as section G96, and it is expected that levels of active muscle 

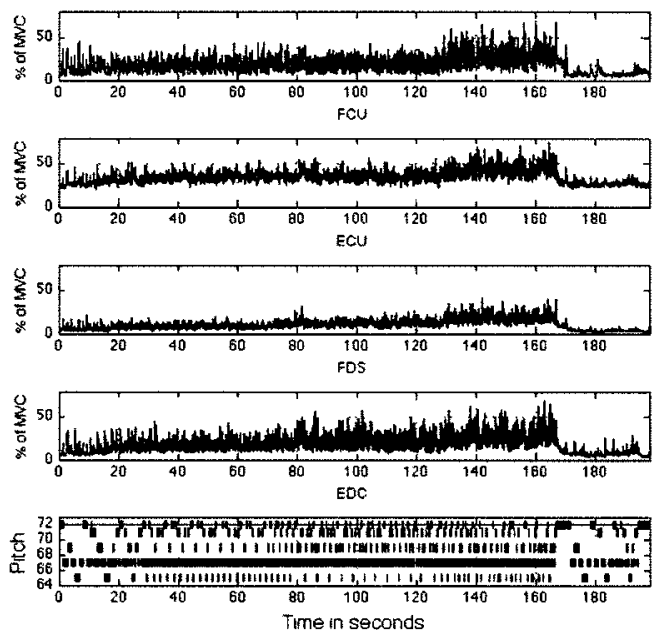

(a) active muscle stiffness - attempt 1
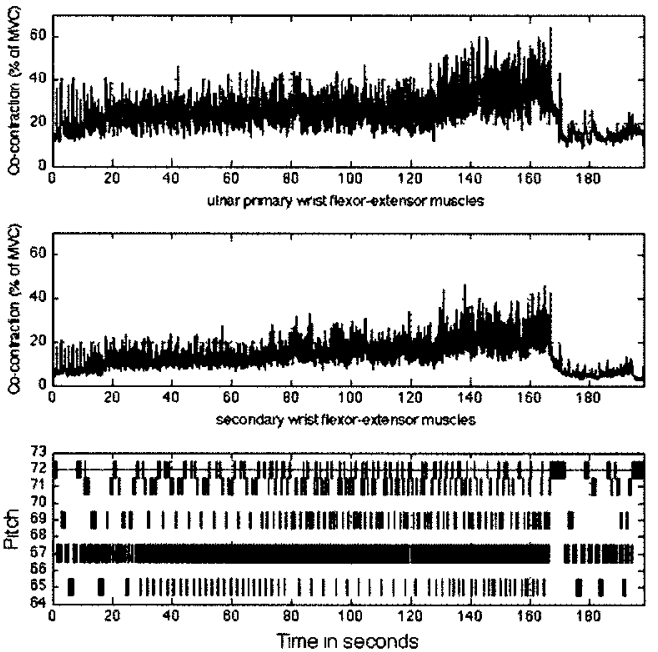

(b) co-contraction - attempt 1

Figure 7.4: Participant E002's results: the upper plots of (a) show active muscle stiffness for the FCU, ECU, FDS and EDC of the right forearm, and the upper plots of (b) show levels of muscular co-contraction while playing the piece by Ann Southam - first attempt. Active muscle stiffness and co-contraction are expressed as a percentage of maximal voluntary contraction. The corresponding MIDI data (note on, note off) are shown on the bottom plots of (a) and (b). 
stiffness and co-contraction were highest in G96, which appears to be confirmed in the plots of active muscle stiffness and co-contraction.) Paired comparisons were made for A and B, A and C96, A and G96, C96 and G96, and G96 and W using a one-way ANOVA. The summary of the ANOVA (with the alpha level of probability set at 0.01 ) of these five comparisons for active muscle stiffness is shown in Table 7.6. It should be noted that in Table 7.6, the results of both attempts of the piece have been pooled. The mean active muscle stiffnesses of the FCU, ECU, FDS and EDC in eight sections of the piece for each participant can be found in Tables Q.1, Q.2, Q.3 and Q.4 of Appendix Q. As was expected, the majority of the time, the mean active muscle stiffness increased as the participants progressed through the piece, and then decreased from section G96 to W.

The same was true of co-contraction. The summary of the ANOVA (with the alpha level of significance set at 0.01) of these five comparisons for CC1 and CC2 is shown in Table 7.7. It should be noted that in Table 7.7, the results of both attempts of the piece have been pooled. Tables Q.5, Q.6, Q.7 and Q.8 of Appendix Q show the means of $\mathrm{CC} 1$ and $\mathrm{CC} 2$ in eight sections of the Ann Southam piece for each participant.

\section{Hypothesis AS-BE}

It was also hypothesized that EMG levels in sections $\mathrm{A}, \mathrm{B}$ and $\mathrm{W}$ would significantly differ from sections A', B' and W', respectively, even though the note sequence, note rate and loudness (with the exception of $W$ and $W^{\prime}$ ) are the same. It was further hypothesized that active muscle stiffness and co-contraction would be higher in sections A' and B' than in sections A and B, respectively, because sections A' and B' are preceded by a passage that is played loudly and has a high note rate. Similarly, 
Table 7.6: Summary of one-way ANOVA tests comparing mean active muscle stiffness of the FCU, ECU, FDS and EDC in sections A, B, C96, G96 and W of the Ann Southam piece. Five pairs of comparisons were made: sections A to B, A to C96, A to G96, C96 to G96 and G96 to W. The total number of participants where the mean of a section labelled " $x$ " was significantly larger than the mean of a section labelled " $\mathrm{y}$ " is indicated in the $\mathrm{x}>\mathrm{y}$ column. The total number of participants where the mean of a section labelled " $x$ " was significantly smaller than the mean of a section labelled " $\mathrm{y}$ " is indicated in the $\mathrm{x}<\mathrm{y}$ column. The total number of participants where the means were not significantly different is indicated in the column titled $\mathrm{x}=\mathrm{y}$. Note, the results of both attempts of the Ann Southam piece have been pooled. The alpha level of probability was set at 0.01 .

\begin{tabular}{|c|c|c|c|c|c|c|}
\hline \multirow[b]{3}{*}{$\mathbf{x}$ to $\mathbf{y}$} & \multicolumn{3}{|c|}{ FCU } & \multicolumn{3}{|c|}{ ECU } \\
\hline & \multirow[b]{2}{*}{$\mathrm{x}=\mathrm{y}$} & \multicolumn{2}{|c|}{$\mathbf{x} \neq \mathbf{y}$} & \multirow[b]{2}{*}{$\mathbf{x}=\mathbf{y}$} & \multicolumn{2}{|c|}{$\mathbf{x} \neq \mathbf{y}$} \\
\hline & & $x>y$ & $\mathbf{x}<\mathbf{y}$ & & $x>y$ & $\mathbf{x}<\mathrm{y}$ \\
\hline$A$ to $B$ & 3 & 3 & 12 & 3 & 0 & 15 \\
\hline $\mathrm{A}$ to $\mathrm{C}$ & 3 & 1 & 14 & 2 & 0 & 16 \\
\hline A to $\mathrm{G}$ & 1 & 1 & 16 & 1 & 1 & 16 \\
\hline $\mathrm{C}$ to $\mathrm{G}$ & 2 & 2 & 14 & 3 & 1 & 14 \\
\hline \multirow[t]{3}{*}{$G$ to $W$} & 4 & 13 & 1 & 0 & 18 & 0 \\
\hline & \multicolumn{3}{|c|}{ FDS } & \multicolumn{3}{|c|}{$\overline{\mathrm{EDC}}$} \\
\hline & & \multicolumn{2}{|c|}{$\mathbf{x} \neq \mathbf{y}$} & & \multicolumn{2}{|c|}{$x \neq y$} \\
\hline $\mathrm{x}$ to $\mathrm{y}$ & $x=y$ & $x>y$ & $\mathbf{x}<\mathbf{y}$ & $\mathbf{x}=\mathbf{y}$ & $x>y$ & $\mathbf{x}<\mathrm{y}$ \\
\hline$A$ to $B$ & 4 & 0 & 14 & 3 & 0 & 15 \\
\hline A to $\mathrm{C}$ & 1 & 0 & 17 & 0 & 0 & 18 \\
\hline A to $G$ & 0 & 0 & 18 & 1 & 0 & 17 \\
\hline $\mathrm{C}$ to $\mathrm{G}$ & 2 & 2 & 14 & 4 & 1 & 13 \\
\hline$G$ to $W$ & 5 & 13 & 0 & 1 & 17 & 0 \\
\hline
\end{tabular}


Table 7.7: Summary of one-way ANOVA tests comparing mean co-contraction (CC1 and $\mathrm{CC} 2$ ) in sections A, B, C96, G96 and W of the Ann Southam piece. Five comparisons were made: sections A to B, A to C96, A to G96, C96 to G96 and G96 to $\mathrm{W}$. The total number of participants where the mean of a section labelled " $\mathrm{x}$ " was significantly larger than the mean of a section labelled " $y$ " is indicated in the $\mathrm{x}>\mathrm{y}$ column. The total number of participants where the mean of a section labelled " $\mathrm{x}$ " was significantly smaller than the mean of a section labelled " $\mathrm{y}$ " is indicated in the $\mathrm{x}<\mathrm{y}$ column. The total number of participants where the means were not significantly different is indicated in the column titled $\mathrm{x}=\mathrm{y}$. Note, the results of both attempts of the Ann Southam piece have been pooled. The alpha level of probability was set at 0.01 .

\begin{tabular}{|c|c|c|c|c|c|c|}
\hline \multirow[b]{3}{*}{$\mathrm{x}$ to $\mathrm{y}$} & \multicolumn{3}{|c|}{ CC1 } & \multicolumn{3}{|c|}{$\mathrm{CC} 2$} \\
\hline & \multirow[b]{2}{*}{$x=y$} & \multicolumn{2}{|c|}{$\mathbf{x} \neq \mathbf{y}$} & \multirow[b]{2}{*}{$\mathrm{x}=\mathrm{y}$} & \multicolumn{2}{|c|}{$\mathbf{x} \neq \mathbf{y}$} \\
\hline & & $x>y$ & $\mathrm{x}<\mathrm{y}$ & & $x>y$ & $\mathbf{x}<\mathbf{y}$ \\
\hline $\mathrm{A}$ to $\mathrm{B}$ & 3 & 2 & 13 & 4 & 0 & 14 \\
\hline $\mathrm{A}$ to $\mathrm{C}$ & 3 & 1 & 14 & 0 & 0 & 18 \\
\hline$A$ to $G$ & 1 & 1 & 16 & 0 & 0 & 18 \\
\hline $\mathrm{C}$ to $\mathrm{G}$ & 3 & 1 & 14 & 2 & 1 & 15 \\
\hline$G$ to $W$ & 0 & 18 & 0 & 1 & 17 & 0 \\
\hline
\end{tabular}


it was hypothesized that active muscle stiffness and co-contraction would be higher in section $\mathrm{W}$ than in $\mathrm{W}^{\prime}$ because section $\mathrm{W}$ immediately follows a passage of music that is played loudly and has a high note rate. To determine if this were true, paired comparisons were made for $\mathrm{A}$ to A', B to B' and W to W' using a one-way ANOVA. Paired comparisons were also made for A to B and A' to B' using a one-way ANOVA, as it was expected these sections would be significantly different. The summary of the ANOVA (with the alpha level of probability set at 0.05 ) of these five comparisons for active muscle stiffness and co-contraction are shown in Table 7.8 and Table 7.9, respectively. It should be noted that in Table 7.8 and Table 7.9 , the results of both attempts of the piece have been pooled.

Similar to what was expected, mean active muscle stiffness of the FCU was significantly greater in A' than in A in more than half of all cases (10 of 18); conversely, the mean active muscle stiffnesses of the ECU, FDS and EDC were greater in A' than in $\mathrm{A}$ in 5 of 18,6 of 18 and 5 of 18 cases, respectively. Comparing B to B', there was no significant difference in mean active muscle stiffnesses of the FCU, FDS and EDC in approximately half of all cases (9 of 18,8 of 18 and 10 of 18 , respectively). In addition, approximately the same number of participants exhibited higher mean active muscle stiffnesses of the FCU, FDS and EDC in B as participants who exhibited higher mean active muscle stiffnesses of the FCU, FDS and EDC in B'. Comparing W to W', the opposite was found: in most cases the mean active muscle stiffnesses of the FCU, ECU, FDS and EDC were significantly higher in W than in W' in 12 of 18,11 of 18,10 of 18 and 11 of 18 cases, respectively.

Co-contraction results were similar to active muscle stiffness results. There was no obvious pattern in co-contraction when comparing $\mathrm{A}$ to $\mathrm{A}^{\prime}$ and $\mathrm{B}$ to $\mathrm{B}$ ':

- Mean CC1 was significantly greater in A' than in A in 7 of 18 cases; mean CC1 
Table 7.8: Summary of one-way ANOVA tests comparing mean active muscle stiffnesses of the FCU, ECU, FDS and EDC of the Ann Southam piece. Five comparisons were made: sections $\mathrm{A}$ to $\mathrm{A}^{\prime}, \mathrm{B}$ to $\mathrm{B}^{\prime}, \mathrm{W}$ to $\mathrm{W}^{\prime}, \mathrm{A}$ to $\mathrm{B}$ and $\mathrm{A}^{\prime}$ to $B$ '. The total number of participants where the mean of a section labelled " $x$ " was significantly larger than the mean of a section labelled " $y$ " is indicated in the $x>y$ column. The total number of participants where the mean of a section labelled " $x$ " was significantly smaller than the mean of a section labelled " $y$ " is indicated in the $\mathrm{x}<\mathrm{y}$ column. The total number of participants where the means were not significantly different is indicated in the column titled $x=y$. Note, the results of both attempts of the Ann Southam piece have been pooled. The alpha level of probability was set at 0.05 .

\begin{tabular}{|c|c|c|c|c|c|c|}
\hline \multirow[b]{3}{*}{$x$ to $y$} & \multicolumn{3}{|c|}{ FCU } & \multicolumn{3}{|c|}{ ECU } \\
\hline & \multirow[b]{2}{*}{$x=y$} & \multicolumn{2}{|c|}{$\mathbf{x} \neq \mathbf{y}$} & \multirow[b]{2}{*}{$x=y$} & \multicolumn{2}{|c|}{$\mathbf{x} \neq \mathbf{y}$} \\
\hline & & $x>y$ & $\mathrm{x}<\mathrm{y}$ & & $x>y$ & $x<y$ \\
\hline A to $A^{\prime}$ & 5 & 3 & 10 & 5 & 8 & 5 \\
\hline $\mathrm{B}^{\prime}$ to $\mathrm{B}^{\prime}$ & 9 & 4 & 5 & 7 & 7 & 4 \\
\hline $\mathrm{W}$ to $\mathrm{W}^{\prime}$ & 3 & 12 & 3 & 4 & 11 & 3 \\
\hline$A$ to $B$ & 3 & 3 & 12 & 1 & 2 & 15 \\
\hline $\mathrm{A}^{\prime}$ to $\mathrm{B}^{\prime}$ & 5 & 5 & 8 & 5 & 1 & 12 \\
\hline \multirow[b]{3}{*}{$x$ to $y$} & \multicolumn{3}{|c|}{ FDS } & \multicolumn{3}{|c|}{ EDC } \\
\hline & \multirow[b]{2}{*}{$x=y$} & \multicolumn{2}{|c|}{$x \neq y$} & \multirow[b]{2}{*}{$x=y$} & \multicolumn{2}{|c|}{$x \neq y$} \\
\hline & & $x>y$ & $\mathrm{x}<\mathrm{y}$ & & $x>y$ & $\mathrm{x}<\mathrm{y}$ \\
\hline A to $A^{\prime}$ & 8 & 4 & 6 & 9 & 4 & 5 \\
\hline $\mathrm{B}$ to $\mathrm{B}^{\prime}$ & 8 & 5 & 5 & 10 & 4 & 4 \\
\hline $\mathrm{W}$ to $\mathrm{W}^{\prime}$ & 5 & 10 & 3 & 4 & 11 & 3 \\
\hline $\mathrm{A}$ to $\mathrm{B}$ & 3 & 1 & 14 & 2 & 0 & 16 \\
\hline$A^{\prime}$ to $B^{\prime}$ & 4 & 0 & 14 & 4 & 1 & 13 \\
\hline
\end{tabular}


Table 7.9: Summary of one-way ANOVA tests comparing co-contraction (CC1 and CC2) in sections $A, B, W, A^{\prime}, B^{\prime}$ and $W^{\prime}$ of the Ann Southam piece. Five comparisons were made: sections $\mathrm{A}$ to $\mathrm{A}^{\prime}, \mathrm{B}$ to $\mathrm{B}^{\prime}, \mathrm{W}$ to $\mathrm{W}^{\prime}, \mathrm{A}$ to $\mathrm{B}$ and $\mathrm{A}^{\prime}$ to $B$ '. The total number of participants where the mean of a section labelled " $\mathrm{x}$ " was significantly larger than the mean of a section labelled " $y$ " is indicated in the $\mathrm{x}>\mathrm{y}$ column. The total number of participants where the mean of a section labelled " $x$ " was significantly smaller than the mean of a section labelled " $y$ " is indicated in the $\mathrm{x}<\mathrm{y}$ column. The total number of participants where the means were not significantly different is indicated in the column titled $\mathrm{x}=\mathrm{y}$. Note, the results of both attempts of the Ann Southam piece have been pooled. The alpha level of probability was set at 0.05 .

\begin{tabular}{|c|c|c|c|c|c|c|}
\hline & \multicolumn{3}{|c|}{ CC1 } & \multicolumn{3}{c|}{ CC2 } \\
\cline { 3 - 4 } & & \multicolumn{2}{|c|}{$\mathbf{x} \neq \mathbf{y}$} & & \multicolumn{2}{c|}{$\mathbf{x} \neq \mathbf{y}$} \\
\cline { 3 - 4 } \cline { 6 - 7 } $\mathbf{x}$ to $\mathbf{y}$ & $\mathbf{x}=\mathbf{y}$ & $\mathbf{x}>\mathbf{y}$ & $\mathbf{x}<\mathbf{y}$ & $\mathbf{x}=\mathbf{y}$ & $\mathbf{x}>\mathbf{y}$ & $\mathbf{x}<\mathbf{y}$ \\
\hline \hline A to A' & 6 & 5 & 7 & 8 & 4 & 6 \\
\hline B to B' & 6 & 6 & 6 & 7 & 6 & 5 \\
\hline W to $\mathbf{W}^{\prime}$ & 3 & 12 & 3 & 4 & 12 & 2 \\
\hline A to B & 2 & 2 & 14 & 1 & 0 & 17 \\
\hline A' to B' $^{\prime}$ & 7 & 2 & 9 & 5 & 0 & 13 \\
\hline
\end{tabular}


was significantly greater in $\mathrm{A}$ than in $\mathrm{A}^{\prime}$ in 5 of 18 cases; mean $\mathrm{CC} 1$ in $\mathrm{A}$ and A' was not significantly different in 6 of 18 cases.

- Mean CC1 was significantly greater in B' than in B in 6 of 18 cases; mean CC1 was significantly greater in $\mathrm{B}$ than in $\mathrm{B}^{\mathrm{\prime}}$ in 6 of 18 cases; mean $\mathrm{CC} 1$ in $\mathrm{B}$ and B' was not significantly different in 6 of 18 cases.

- Mean CC2 was significantly greater in $\mathrm{A}^{\prime}$ than in A in 6 of 18 cases; mean CC2 was significantly greater in A than in $\mathrm{A}^{\prime}$ in 4 of 18 cases; mean $\mathrm{CC} 2$ in $\mathrm{A}$ and A' was not significantly different in 8 of 18 cases.

- Mean CC2 was significantly greater in B' than in B in 5 of 18 cases; mean CC2 was significantly greater in B than in B' in 6 of 18 cases; mean $\mathrm{CC} 2$ in B and B' was not significantly different in 7 of 18 cases.

Thus, parts a and b of hypothesis AS-BE are rejected. The comparison of $\mathrm{W}$ to $\mathrm{W}$ ' showed that mean co-contraction was higher in $\mathrm{W}$ than in $\mathrm{W}^{\prime}$ in most cases - 12 of 18 for $\mathrm{CC} 1$ and 12 of 18 for $\mathrm{CC} 2$. This confirms part $\mathrm{c}$ of hypothesis AS-BE.

The comparisons of $\mathrm{A}$ to $\mathrm{B}$ and $\mathrm{A}$ ' to $\mathrm{B}$ ' were done to verify that the mean active muscle stiffnesses and levels of co-contraction were not significantly different from A to B and from A' to B'. In most cases, mean active muscle stiffness and co-contraction were significantly greater in B than in A; similarly, in most cases mean active stiffness and co-contraction were significantly greater in B' than in A'. Had it been found that mean active muscle stiffness and co-contraction were not significantly different, the results could have been pooled; however, this was not the case. 


\subsection{Discussion}

\subsubsection{Execution of Piece by Ann Southam}

For the most part, the Ann Southam piece was well-executed, although no participant performed the piece perfectly. The participants were evaluated on their note accuracy, use of dynamics (in the musical sense of the word), and tempo.

Note accuracy errors were classified as addition, deletion or substitution errors. By far the majority of note accuracy errors occurred in the middle of the piece (i.e. in bars 9 to 20 inclusively, which correspond to sections D, E, F and G). Participants E006, E007, E008 and E009 made the most note accuracy errors during a first attempt of the piece. In all cases except that of E002, participants made fewer note accuracy errors the second time they played the piece. Similarly, participants made fewer repetition and omission errors the second time they played the piece, with the exception of participants E004 and E008, who made more repetition errors.

The one aspect where all participants did not adequately fulfill the requirements of the piece was in the contrast of the dynamics. None of the participants had mean key velocities greater than 90, which qualifies as forte according to [58]. Forte is not as loud as fortisissimo, which is the dynamic level indicated in section W.

There were variations observed in the tempo. In some cases, participants initially played faster than the set tempo in sections A, B and C96, but slowed down in section G96 (e.g. E004 attempt 1); in other cases, participants started slower than the set tempo, gradually played faster, and played section G96 above tempo (e.g. E007 attempt 2). In both attempts of the piece, participants E002, E007 and E009

played much slower than the set tempo in sections $\mathrm{A}$ and $\mathrm{B}$, but played above tempo in section G96 (except for participant E009's attempt 1). 


\subsubsection{EMG Results and Hypothesis AS-SC}

One of the primary goals of this study was to observe if a change in stiffness took place from section A to section G96. In her study, which used the same piece of music, but detected changes in mechanical impedance using a driving point impedance model, Vant detected no change in stiffness from section A to section $\mathrm{C}$. The results of this study indicate otherwise.

Our study uses changes of active muscle stiffness and co-contraction as indicators of changes in wrist stiffness. Most participants experienced an increase in active muscle stiffness and co-contraction from section A to section $\mathrm{C}$, contrary to what was found in Vant's study. There were increases in active muscle stiffness and cocontraction from $\mathrm{A}$ to $\mathrm{B}$, from $\mathrm{A}$ to $\mathrm{C} 96$, from $\mathrm{A}$ to $\mathrm{G} 96$ and from C96 to G96 in at least two thirds of cases. This shows that there is a general increase in active muscle stiffness and co-contraction from the start of section A to the end of section G96. While not all participants played at tempo, they all ${ }^{2}$ demonstrated a greater note rate in section $\mathrm{B}$ than in $\mathrm{A}$, a greater note rate in C96 than in B, and a greater note rate in G96 than in C96, which means note rate increased from A to G. Because not all participants played with as much dynamic contrast as was indicated in the piece, it is suspected that increases in active muscle stiffness and co-contraction are primarily due to increases in note rate. An additional analysis, such as a two-way ANOVA, or a separate study should be conducted to tease apart the relationship between increased note rate and increased loudness with active muscle stiffness and co-contraction. A separate study could investigate the effect of several loudness levels while playing multiple repetitions of the first two bars of the Ann Southam piece.

It was also observed that active muscle stiffness and co-contraction decreased

\footnotetext{
${ }^{2}$ With the exception of E005 and E006, who played with a higher mean note rate in C96 than in G96 in both attempts of the piece.
} 
from section G96 to section W. Section W is typically played louder than section G96 (although not necessarily significantly louder), but its note rate is one-sixteenth that of section G96. These findings support the idea that active muscle stiffness and co-contraction are dependent on note rate, but are unclear about the relationship of loudness (expressed as key velocity in the collected MIDI data) to active muscle stiffness and to co-contraction.

\subsubsection{EMG Results and Hypothesis AS-BE}

After Ann Southam composed the music for Vant's study, the first four bars of the piece were duplicated and added to the end of the composition so that comparisons could be made between the beginning and end of the piece. It was expected that there would be an increase in active muscle stiffness and co-contraction from section A to section A' and from section $B$ to section $B^{\prime}$ because sections A' and B' follow bars 9 through 20, where the note rate of the piece is highest. It was hypothesized that previous high levels of active muscle stiffness and co-contraction would affect a person's ability to play with lower active muscle stiffness and co-contraction levels.

Although there is an increase in active muscle stiffness and co-contraction from A to G96, active muscle stiffness and co-contraction levels in section A' returned to those observed during section A. The same was true for sections B and B'. Thus, an interval of increased active muscle stiffness and co-contraction appears to have no significant effect on the levels of active muscle stiffness and co-contraction observed in the immediately succeeding interval (i.e. in sections A' and B').

The same cannot be said for sections $W$ and W'. In nearly two-thirds of cases the mean active muscle stiffness and co-contraction were greater in section $\mathrm{W}$ than in section $W^{\prime}$. Section $W$ occurs immediately after an interval of increasing active muscle stiffness and co-contraction, and is played more loudly than section $W^{\prime}$. These 
are likely reasons for the difference observed between the two sections.

As mentioned earlier in this chapter, section $\mathrm{A}$ was compared to section $\mathrm{B}$, and section A' was compared to section B' to observe if there were any significant differences in mean active muscle stiffness and co-contraction. In at least two-thirds of cases, mean active muscle stiffness and co-contraction were significantly greater in section $B$ than in section $A$. The same pattern was observed when sections A' and B' were compared, although there were not as many cases. Sections A and B could have been pooled had there been very few cases where mean active muscle stiffness and co-contraction were significantly different. The same would have been true of sections $A^{\prime}$ and $B^{\prime}$.

\subsection{Outcomes of Hypothesis Testing}

In this chapter, two hypotheses were proposed:

1. Hypothesis AS-SC: Active muscle stiffness and co-contraction will significantly increase from the beginning of the Ann Southam piece to the end of bar 20.

This hypothesis was confirmed.

2. Hypothesis AS-BE: Active muscle stiffness and co-contraction will be significantly different between the beginning and end of the Ann Southam piece.

This hypothesis was split into three more specific hypotheses:

2a. Hypothesis AS-BE: Active muscle stiffness and co-contraction will be significantly different in sections A and A' of the Ann Southam piece.

This hypothesis was rejected. 
2b. Hypothesis AS-BE: Active muscle stiffness and co-contraction will be significantly different in sections B and B' of the Ann Southam piece.

This hypothesis was rejected.

2c. Hypothesis AS-BE: Active muscle stiffness and co-contraction will be significantly different in sections $W$ and $W^{\prime}$ of the Ann Southam piece.

This hypothesis was confirmed. 


\section{Chapter 8}

\section{Summary and Discussion}

In Chapters 5 and 6 it was hypothesized that certain tasks would yield low and high levels of co-contraction; however, categorizing co-contraction as low or high does not appear to occur in the literature, and there appear to be no values assigned to low and high co-contraction levels. In Jonsson's [59] study of muscular endurance during work, he measures EMG levels during different assembly tasks. In his paper, Jonsson refers to "low muscular activity" and high and low static loads, although it is unclear what qualifies as low and high. In Østensvik et al.'s 2009 paper [60] on the evaluation of muscle activity patterns associated with musculoskeletal discomfort, they defined a sustained low-level muscle activity as a sustained contraction above $0.5 \%$ MVC over a length of time at least $1.6 \mathrm{~s}$ long [60]. While playing the piano can be a sustained task, levels of mean active muscle stiffness were, in almost all cases, much higher than $0.5 \%$ MVC. In fact, the lowest values of mean active muscle stiffness and co-contraction in this study were commonly observed in section A of the Ann Southam piece, where mean values varied between $.66 \% \mathrm{MVC}$ and $36.94 \% \mathrm{MVC}$, and were typically around $10 \%$ MVC to $11 \%$ MVC. As previously mentioned in Section 5.2.2 and Section 6.2.3, the high values of mean active muscle stiffness and co-contraction might be a result of the normalization methods used in this study. Our normalization methods should 
be reevaluated, and this will be discussed in Section 8.2 .

\subsection{Summary of Results}

The following plots were generated to visualize and estimate how active muscle stiffness and co-contraction levels compare to one another across tasks. Included on the plots are the pooled ascending and pooled descending segments of the $\mathrm{C}$ major scale, section G96 of both attempts of the Ann Southam piece, and mTRs and mTRe of TR1 and TR2. Since the lowest levels of active muscle stiffness and co-contraction were in section $A$ of the Ann Southam piece the majority of the time, the results are expressed as a fraction of section $\mathrm{A}^{1}$ on the left $\mathrm{y}$ axis and as a percentage of the MVC on the right y axis. The summary plots are purely for speculative observations; no statistical analyses were carried out to compare experimental tasks to one another. For each of the tasks, where a pair of means is significantly different (e.g. where the mean active stiffness of the ascending segment of the $\mathrm{C}$ major scale is statistically larger than the mean of the descending segment), this is marked with a $※$. Results of all nine participants are shown in Figures 8.1, 8.2, 8.3, 8.4, 8.5, 8.6, 8.7, 8.8 and 8.9. Each figure shows the mean active muscle stiffness for all four muscles considered in this study and co-contraction of antagonistic muscle pairs. A $\dagger$ indicates where results of a particular trial were deemed invalid. As noted in Section 5.1.3, there was a problem with the sensor used to detect the activity of participant E006's FCU. This lasted for approximately 8.5 seconds at the start of the recording, and the FCU results (as well as the $\mathrm{CC} 1$ results) for this time, indicated by a $\S$, have been omitted from calculations of the mean.

\footnotetext{
${ }^{1}$ This is actually the mean of section A from attempt 1 and attempt 2 of the Ann Southam piece.
} 
Although never explicitly stated in this document, before any analyses of the results were begun, it was expected that a summary of the results would show that the scale had the lowest levels of active muscle stiffness and co-contraction, followed by section G96 of the Ann Southam piece, followed by mTRe of TR2 then mTRe of TR1. The scale was expected to have the lowest levels of active muscle stiffness and co-contraction based on the findings of Osu et al. [26], which state that joint viscoelasticity, as indicated by the index of muscle co-contraction around the joint (IMCJ), was high during learning of new movements, but as task performance improved, IMCJ decreased. The assumption was that the $\mathrm{C}$ major scale is a task with which advanced pianists should be quite familiar. The two triad exercises were chosen to be included in this study because they require coordinated finger movements. Participants experienced discomfort when executing the triad exercises, some noting they perceived a stiffening of the wrist, especially during TR1. Comments regarding discomfort or perceived wrist stiffening were not made following the scale nor the Ann Southam piece. For this reason, active muscle stiffness and co-contraction were expected to be highest during the triad exercises. The mean active muscle stiffness and co-contraction levels during section G96 of the Ann Southam piece were expected to fall somewhere between those of the scale and those of the triads. The pattern of scale < section G96 in the Ann Southam puece < triad exercuses is not observed in Figures $8.1,8.2,8.3,8.4,8.5,8.6,8.7,8.8$ and 8.9 with the exception of participant E003's mean active muscle stiffness of the ECU and mean CC1.

The analyses conducted in Chapters 5, 6 and 7 revealed that mean active muscle stiffness and co-contraction appear to be a function of note rate; thus, lower levels of mean active muscle stiffness and co-contraction should be observed in section G96 of the Ann Southam piece than in the $\mathrm{C}$ major scale and triad exercises. The majority of the results presented in Figures 8.1, 8.2, 8.3, 8.4, 8.5, 8.6, 8.7, 8.8 and 8.9 appear 


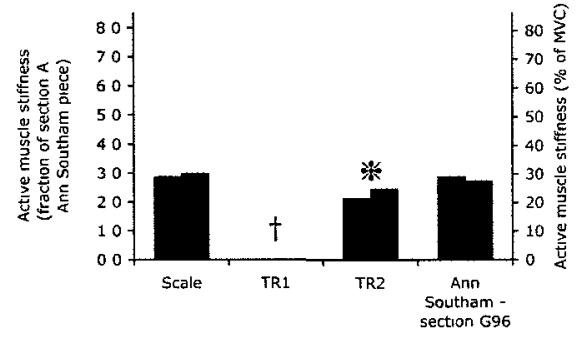

(a) FCU

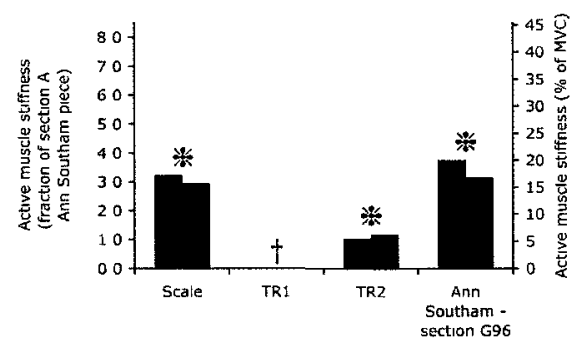

(c) FDS

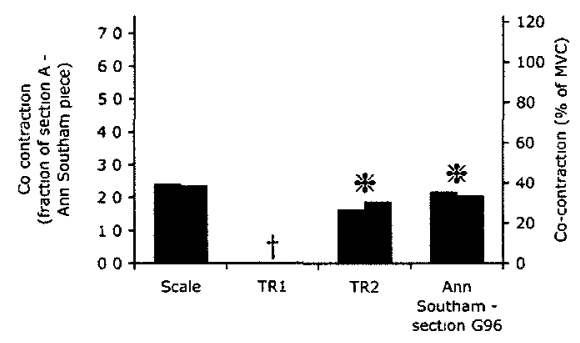

(e) $\mathrm{CC} 1$

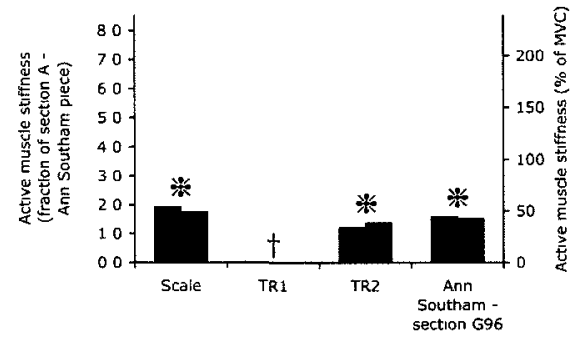

(b) ECU

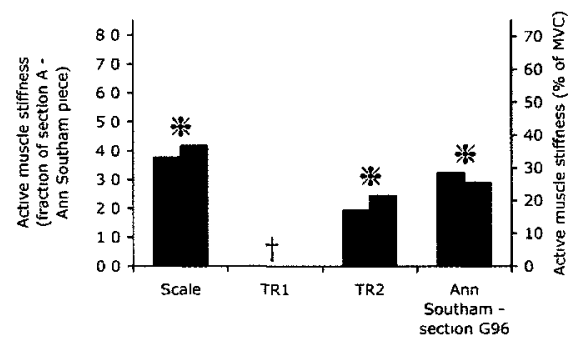

(d) EDC

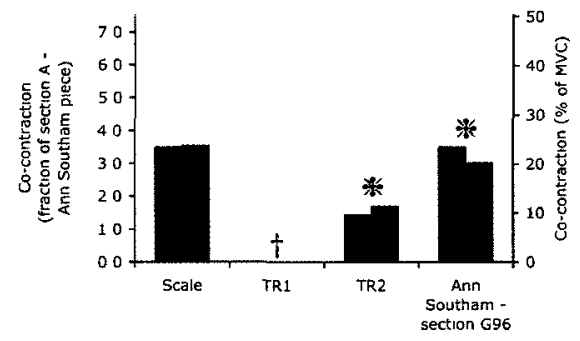

(f) $\mathrm{CC} 2$

Figure 8.1: A summary of participant E002's mean active muscle stiffness and co-contraction results, comparing ascending and descending segments of the $\mathrm{C}$ major scale (blue and red, respectively), mTRs and mTRe of TR1 (blue and red, respectively), mTRs and mTRe of TR2 (blue and red, respectively), and attempt 1 and 2 of section G96 of the Ann Southam piece (blue and red, respectively). Bars in (a), (b), (c) and (d) represent mean active muscle stiffness; bars in (e) and (f) represent mean co-contraction. Mean active muscle stiffness and mean co-contraction are expressed as a fraction of section $\mathrm{A}$ of the Ann Southam piece (left $\mathrm{y}$ axis) and as a percentage of the MVC (right y axis). Results classified as invalid are omitted; this is indicated by a $\uparrow$. A $※$ indicates the pair of results is significantly different, as determined by a one-way ANOVA or Student's t-test, $\mathrm{P}<0.05$. 


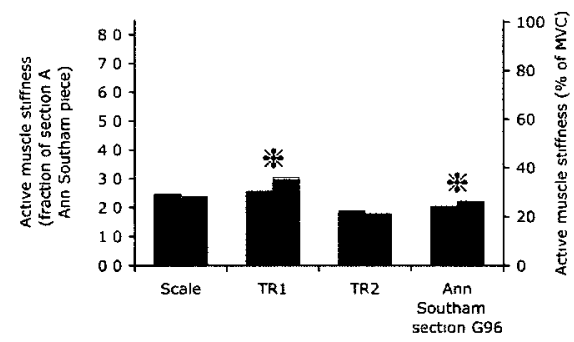

(a) $\mathrm{FCU}$

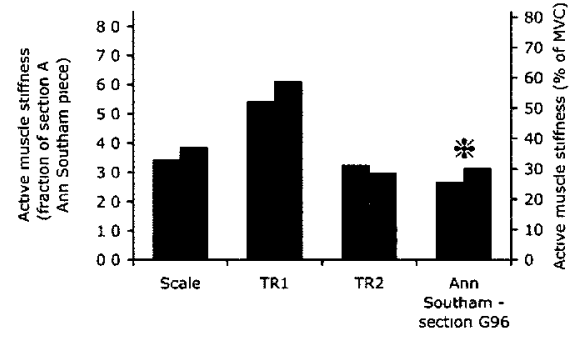

(c) FDS

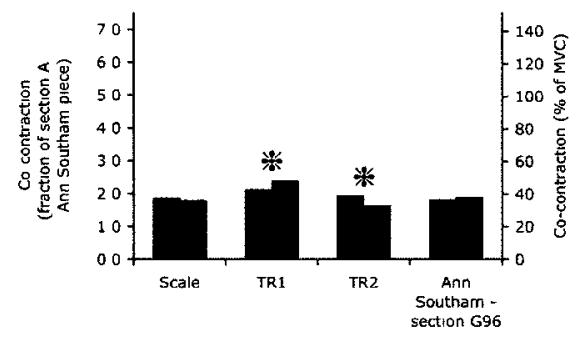

(e) $\mathrm{CC} 1$

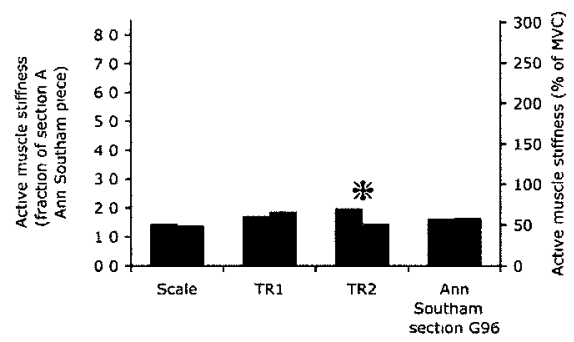

(b) ECU

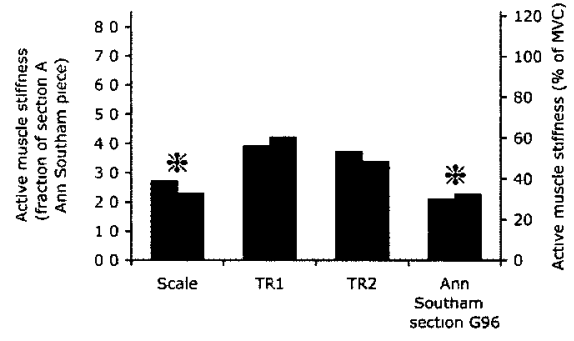

(d) EDC

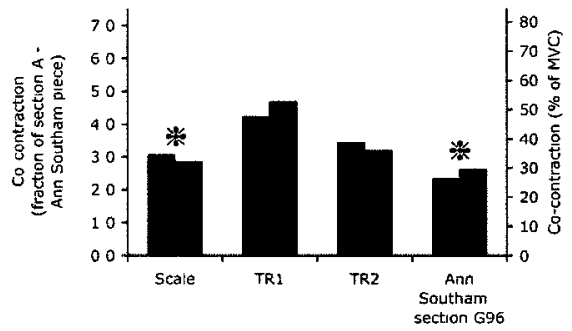

(f) $\mathrm{CC} 2$

Figure 8.2: A summary of participant E003's mean active muscle stiffness and co-contraction results, comparing ascending and descending segments of the $\mathrm{C}$ major scale (blue and red, respectively), mTRs and mTRe of TR1 (blue and red, respectively), mTRs and mTRe of TR2 (blue and red, respectively), and attempt 1 and 2 of section G96 of the Ann Southam piece (blue and red, respectively). Bars in (a), (b), (c) and (d) represent mean active muscle stiffness; bars in (e) and (f) represent mean co-contraction. Mean active muscle stiffness and mean co-contraction are expressed as a fraction of section $A$ of the Ann Southam piece (left y axis) and as a percentage of the MVC (right y axis). A $*$ indicates the pair of results is significantly different, as determined by a one-way ANOVA or Student's t-test, $\mathrm{P}<0.05$. 


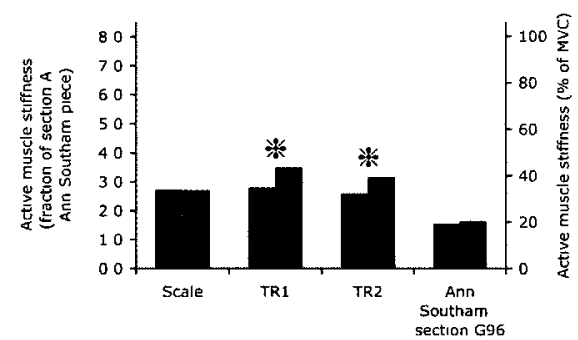

(a) FCU

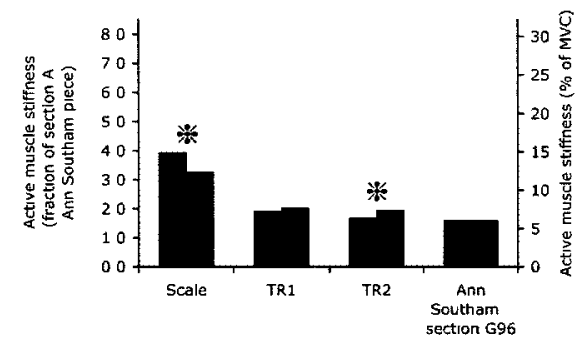

(c) FDS

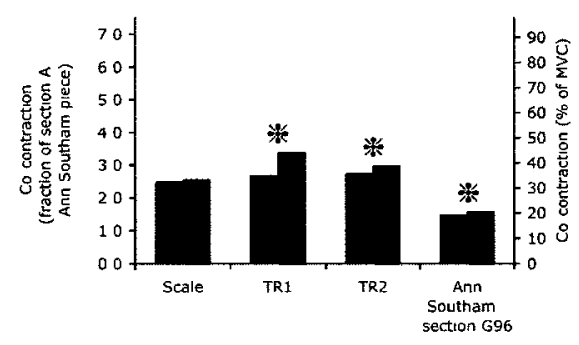

(e) $\mathrm{CC} 1$

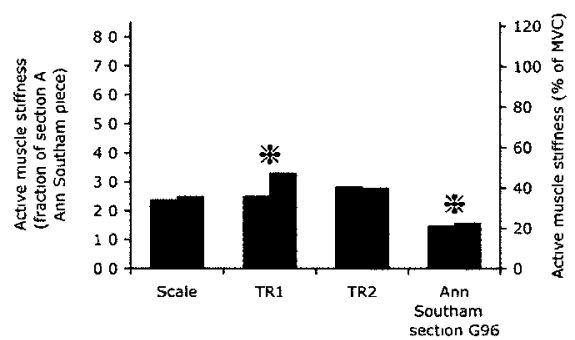

(b) ECU

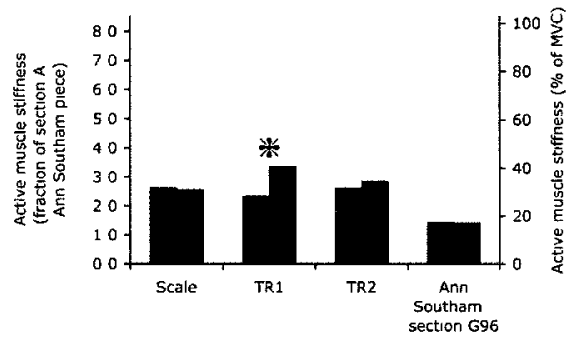

(d) EDC

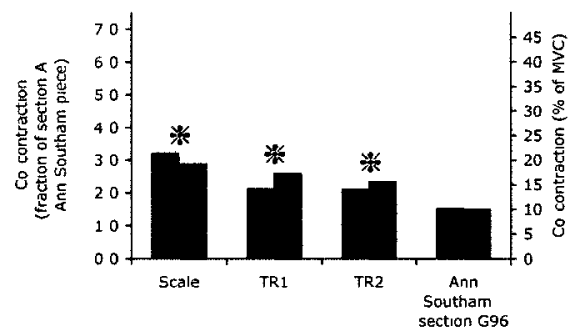

(f) $\mathrm{CC} 2$

Figure 8.3: A summary of participant E004's mean active muscle stiffness and co-contraction results, comparing ascending and descending segments of the $\mathrm{C}$ major scale (blue and red, respectively), mTRs and mTRe of TR1 (blue and red, respectively), mTRs and mTRe of TR2 (blue and red, respectively), and attempt 1 and 2 of section G96 of the Ann Southam piece (blue and red, respectively). Bars in (a), (b), (c) and (d) represent mean active muscle stiffness; bars in (e) and (f) represent mean co-contraction. Mean active muscle stiffness and mean co-contraction are expressed as a fraction of section $\mathrm{A}$ of the Ann Southam piece (left y axis) and as a percentage of the MVC (right y axis). A $\%$ indicates the pair of results is significantly different, as determined by a one-way ANOVA or Student's t-test, $\mathrm{P}<0.05$. 


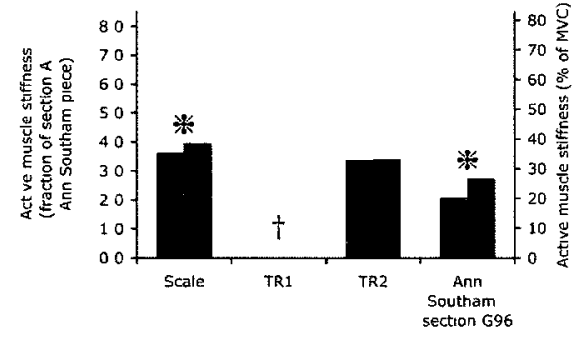

(a) FCU

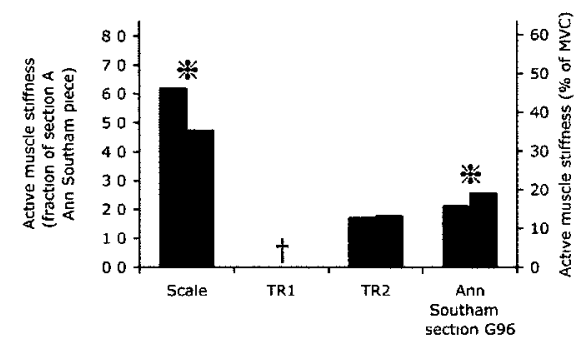

(c) FDS

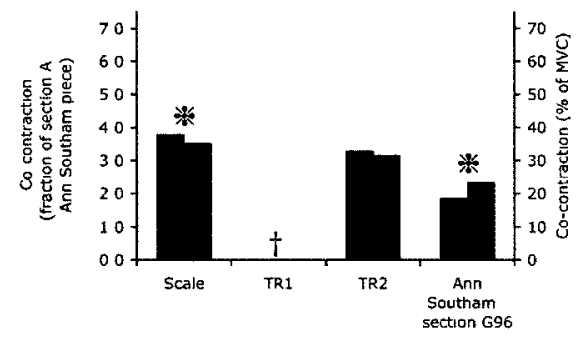

(e) $\mathrm{CC} 1$

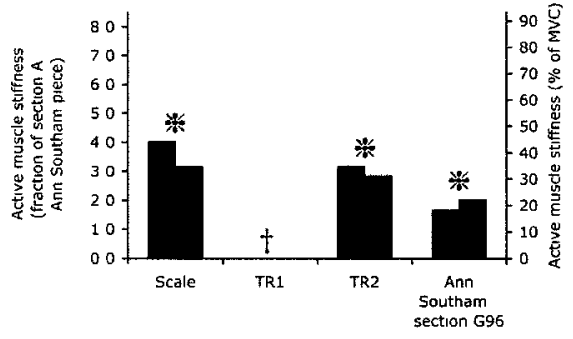

(b) ECU

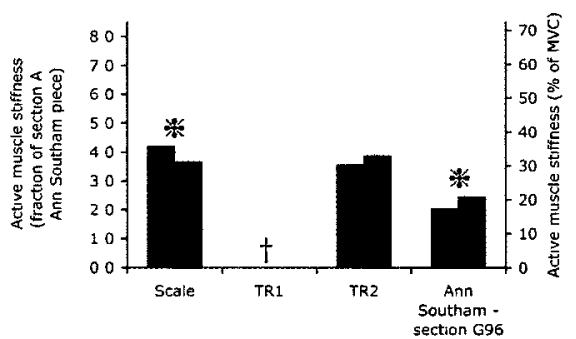

(d) EDC

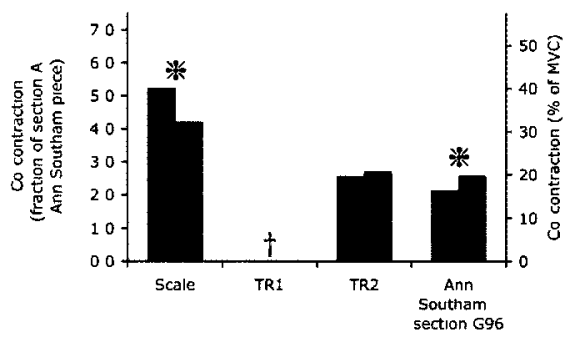

(f) $\mathrm{CC} 2$

Figure 8.4: A summary of participant E005's mean active muscle stiffness and co-contraction results, comparing ascending and descending segments of the $\mathrm{C}$ major scale (blue and red, respectively), mTRs and mTRe of TR1 (blue and red, respectively), mTRs and mTRe of TR2 (blue and red, respectively), and attempt 1 and 2 of section G96 of the Ann Southam piece (blue and red, respectively). Bars in (a), (b), (c) and (d) represent mean active muscle stiffness; bars in (e) and (f) represent mean co-contraction. Mean active muscle stiffness and mean co-contraction are expressed as a fraction of section $\mathrm{A}$ of the Ann Southam piece (left y axis) and as a percentage of the MVC (right y axis). Results classified as invalid are omitted; this is indicated by a $\uparrow$. A $※$ indicates the pair of results is significantly different, as determined by a one-way ANOVA or Student's t-test, $\mathrm{P}<0.05$. 


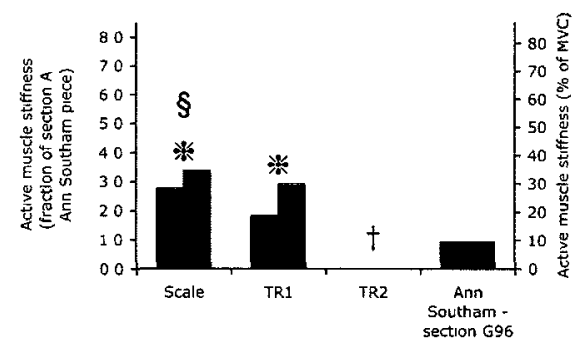

(a) FCU

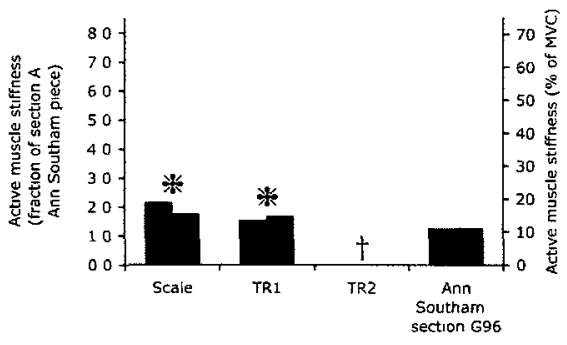

(c) FDS

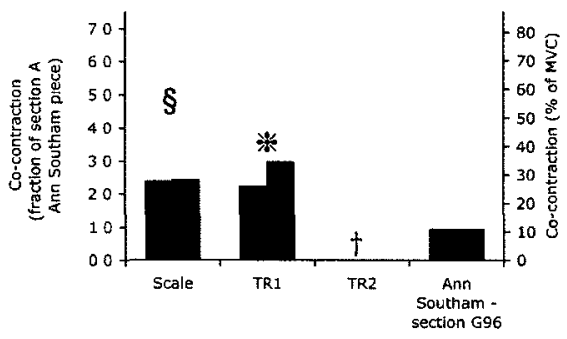

(e) $\mathrm{CCl}$

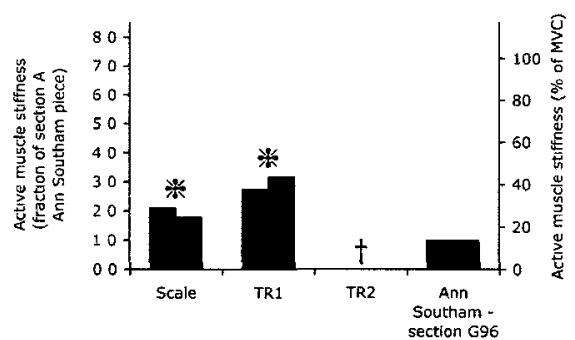

(b) ECU

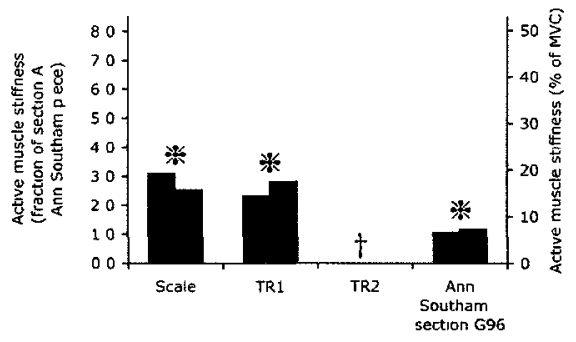

(d) EDC

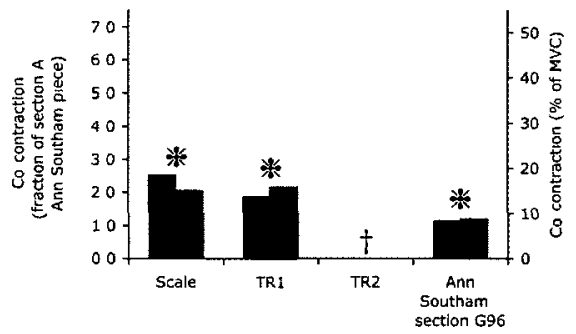

(f) $\mathrm{CC} 2$

Figure 8.5: A summary of participant E006's mean active muscle stiffness and co-contraction results, comparing ascending and descending segments of the $\mathrm{C}$ major scale (blue and red, respectively), mTRs and mTRe of TR1 (blue and red, respectively), mTRs and mTRe of TR2 (blue and red, respectively), and attempt 1 and 2 of section G96 of the Ann Southam piece (blue and red, respectively). Bars in (a), (b), (c) and (d) represent mean active muscle stiffness; bars in (e) and (f) represent mean co-contraction. Mean active muscle stiffness and mean co-contraction are expressed as a fraction of section $\mathrm{A}$ of the Ann Southam piece (left $y$ axis) and as a percentage of the MVC (right y axis). Results classified as invalid are omitted; this is indicated by a $\dagger$. A §indicates where some results were left out of the calculation of the mean due to a problem with the sensor. A $※$ indicates the pair of results is significantly different, as determined by a one-way ANOVA or Student's t-test, $\mathrm{P}<0.05$. 


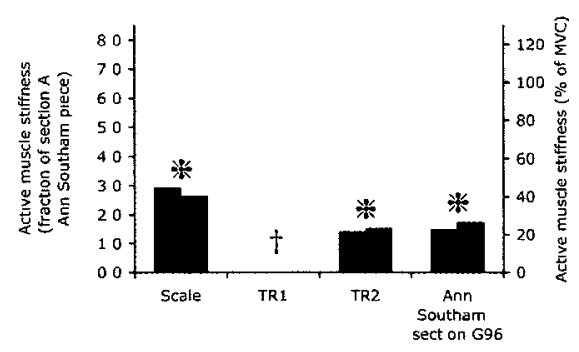

(a) FCU

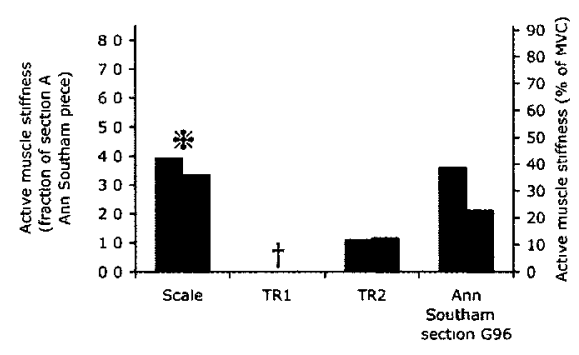

(c) FDS

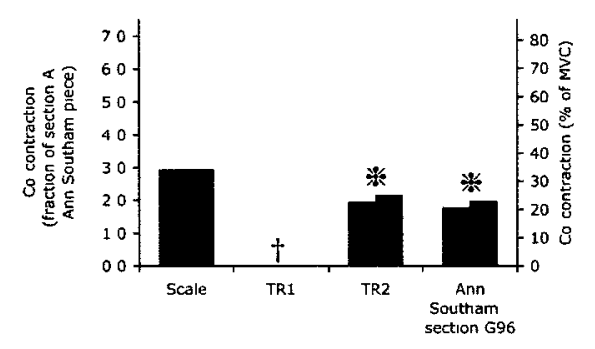

(e) $\mathrm{CC} 1$

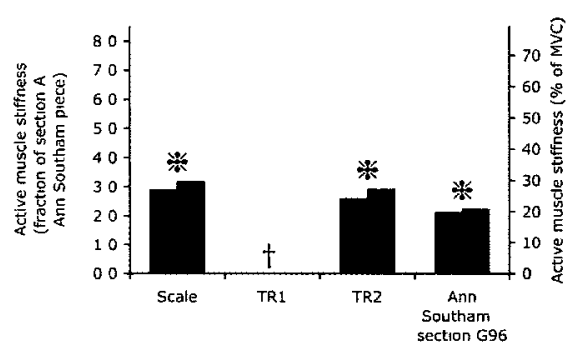

(b) ECU

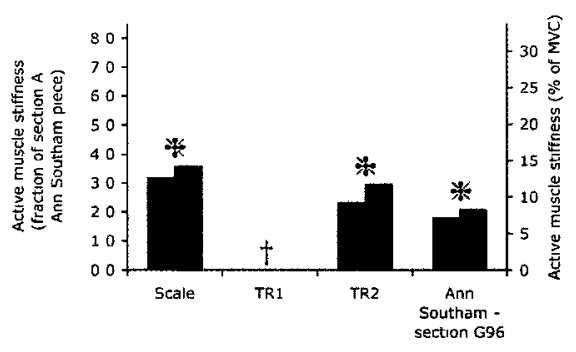

(d) EDC

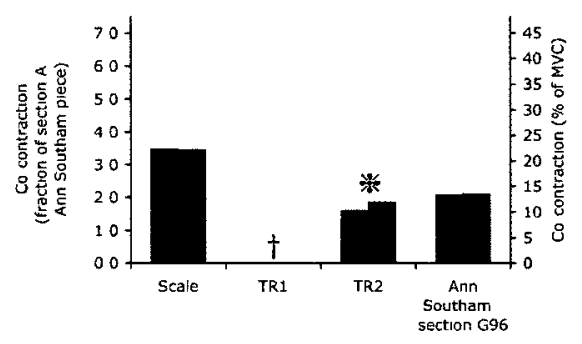

(f) $\mathrm{CC} 2$

Figure 8.6: A summary of participant E007's mean active muscle stiffness and co-contraction results, comparing ascending and descending segments of the $\mathrm{C}$ major scale (blue and red, respectively), mTRs and mTRe of TR1 (blue and red, respectively), mTRs and mTRe of TR2 (blue and red, respectively), and attempt 1 and 2 of section G96 of the Ann Southam piece (blue and red, respectively). Bars in (a), (b), (c) and (d) represent mean active muscle stiffness; bars in (e) and (f) represent mean co-contraction. Mean active muscle stiffness and mean co-contraction are expressed as a fraction of section $\mathrm{A}$ of the Ann Southam piece (left y axis) and as a percentage of the MVC (right y axis). Results classified as invalid are omitted; this is indicated by a $\uparrow$. A indicates the pair of results is significantly different, as determined by a one-way ANOVA or Student's t-test, $\mathrm{P}<0.05$. 


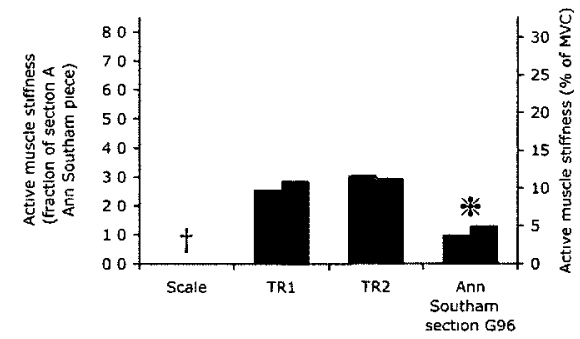

(a) FCU

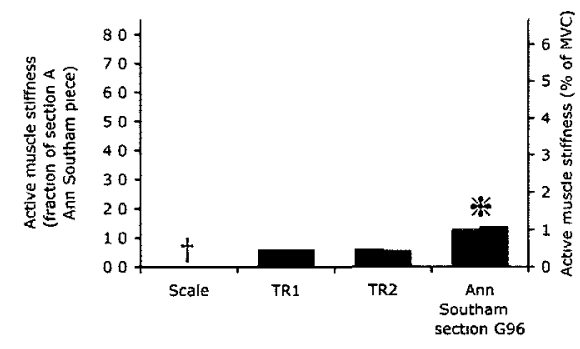

(c) FDS

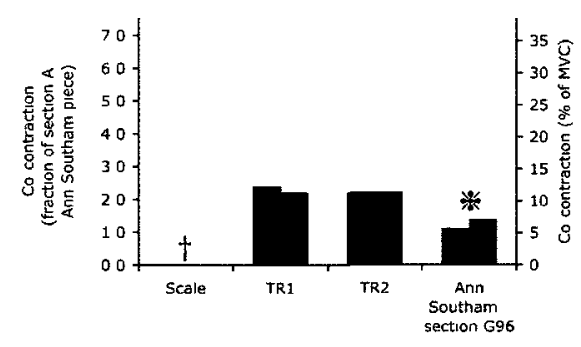

(e) $\mathrm{CC} 1$

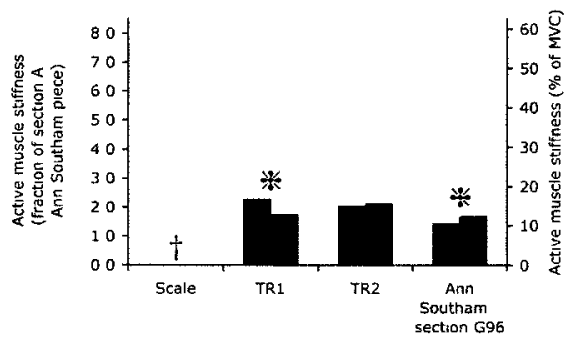

(b) ECU

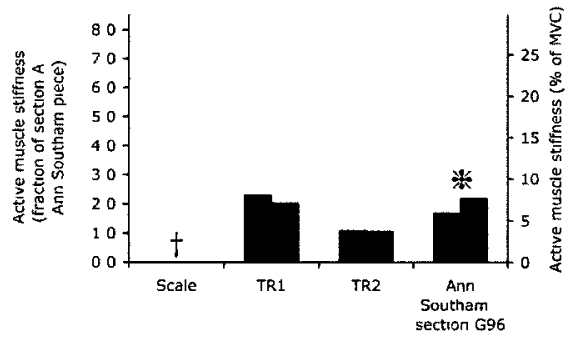

(d) EDC

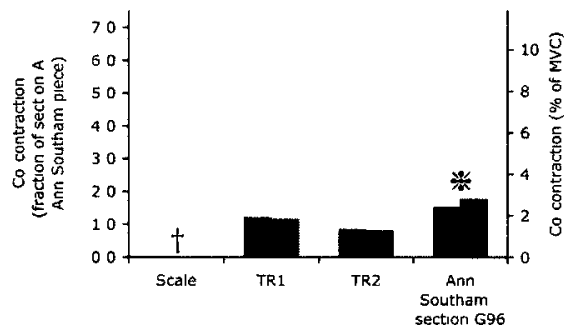

(f) $\mathrm{CC} 2$

Figure 8.7: A summary of participant E008's mean active muscle stiffness and co-contraction results, comparing ascending and descending segments of the $\mathrm{C}$ major scale (blue and red, respectively), mTRs and mTRe of TR1 (blue and red, respectively), mTRs and mTRe of TR2 (blue and red, respectively), and attempt 1 and 2 of section G96 of the Ann Southam piece (blue and red, respectively). Bars in (a), (b), (c) and (d) represent mean active muscle stiffness; bars in (e) and (f) represent mean co-contraction. Mean active muscle stiffness and mean co-contraction are expressed as a fraction of section $A$ of the Ann Southam piece (left y axis) and as a percentage of the MVC (right y axis). A ※ indicates the pair of results is significantly different, as determined by a one-way ANOVA or Student's t-test, $\mathrm{P}<0.05$. 


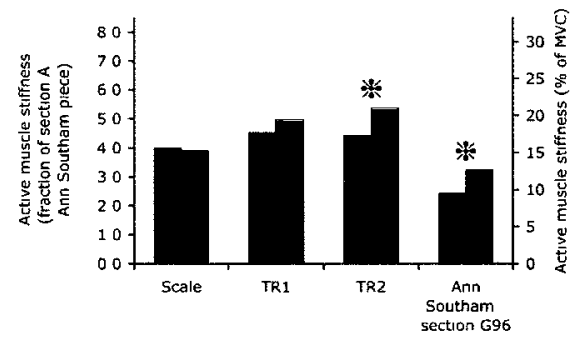

(a) $\mathrm{FCU}$

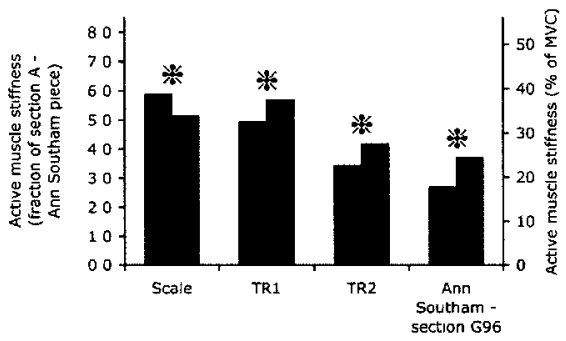

(c) FDS

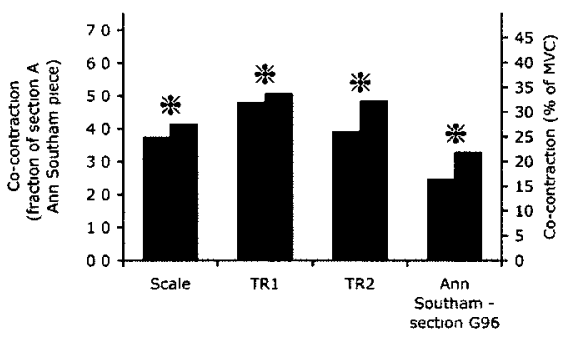

(e) $\mathrm{CC} 1$

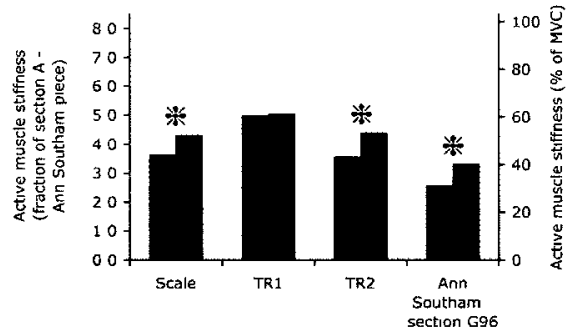

(b) ECU

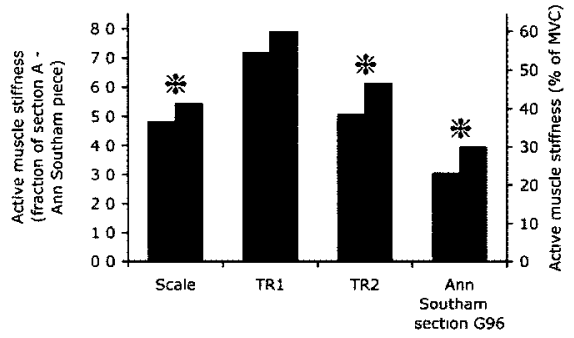

(d) EDC

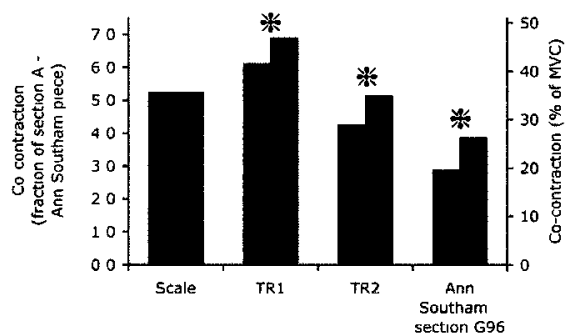

(f) $\mathrm{CC} 2$

Figure 8.8: A summary of participant E009's mean active muscle stiffness and co-contraction results, comparing ascending and descending segments of the $\mathrm{C}$ major scale (blue and red, respectively), mTRs and mTRe of TR1 (blue and red, respectively), mTRs and mTRe of TR2 (blue and red, respectively), and attempt 1 and 2 of section G96 of the Ann Southam piece (blue and red, respectively). Bars in (a), (b), (c) and (d) represent mean active muscle stiffness; bars in (e) and (f) represent mean co-contraction. Mean active muscle stiffness and mean co-contraction are expressed as a fraction of section A of the Ann Southam piece (left y axis) and as a percentage of the MVC (right y axis). A $※$ indicates the pair of results is significantly different, as determined by a one-way ANOVA or Student's t-test, $\mathrm{P}<0.05$. 


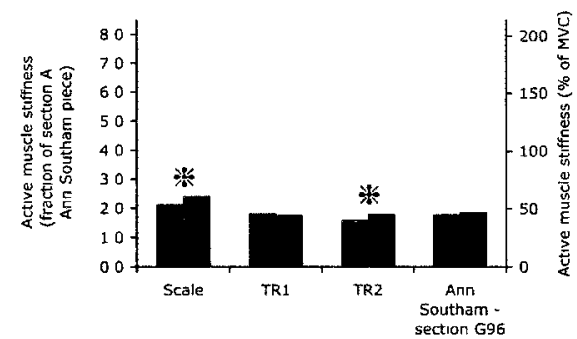

(a) $\mathrm{FCU}$

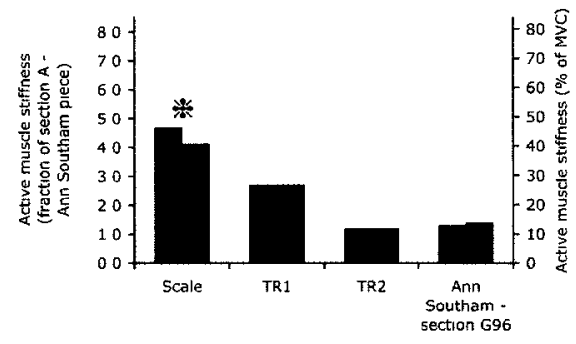

(c) FDS

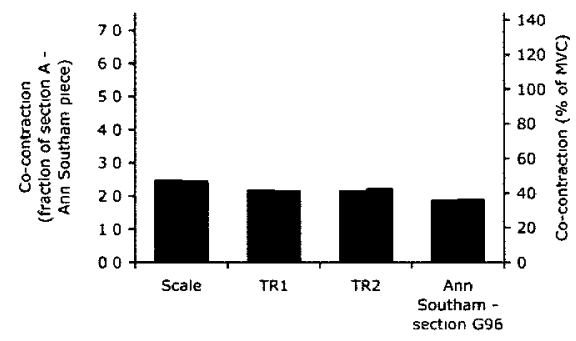

(e) CC1

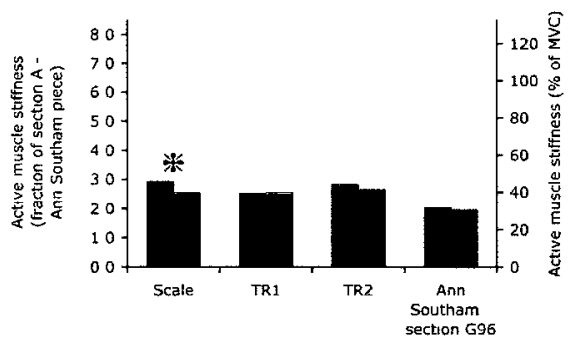

(b) ECU

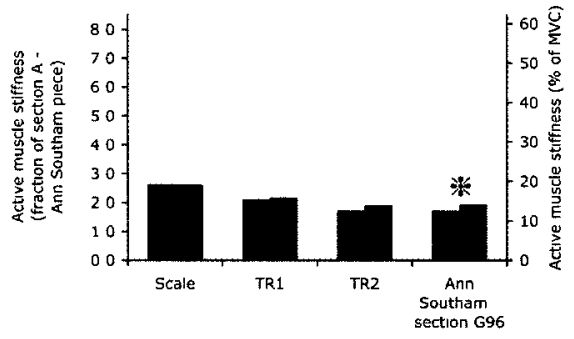

(d) EDC

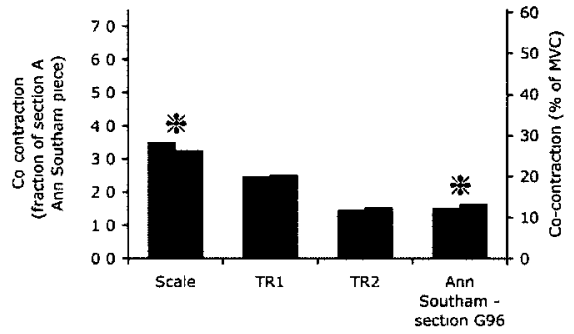

(f) $\mathrm{CC} 2$

Figure 8.9: A summary of participant E010's mean active muscle stiffness and co-contraction results, comparing ascending and descending segments of the $\mathrm{C}$ major scale (blue and red, respectively), mTRs and mTRe of TR1 (blue and red, respectively), mTRs and mTRe of TR2 (blue and red, respectively), and attempt 1 and 2 of section G96 of the Ann Southam piece (blue and red, respectively). Bars in (a), (b), (c) and (d) represent mean active muscle stiffness; bars in (e) and (f) represent mean co-contraction. Mean active muscle stiffness and mean co-contraction are expressed as a fraction of section $A$ of the Ann Southam piece (left y axis) and as a percentage of the MVC (right y axis). A $※$ indicates the pair of results is significantly different, as determined by a one-way ANOVA or Student's t-test, $\mathrm{P}<0.05$. 
to follow this pattern. ${ }^{2}$

Considering only the triad exercises, where participants had valid data from both triad exercises, it appears that mean active muscle stiffness and co-contraction are higher in TR1 than TR2. There are several cases where this is not true:

- in participant E003's ECU mean active muscle stiffness results (mTRs),

- in participant E004's ECU and EDC mean active muscle stiffness results (mTRs),

- in participant E008's FCU mean active muscle stiffness results (mTRs and mTRe), ECU mean active muscle stiffness (mTRe), FDS mean active muscle stiffness (mTRs), CC1 results (mTRe),

- in participant E009's FCU mean active muscle stiffness results (mTRs), and

- in participant E010's FCU mean active muscle stiffness results (mTRe), ECU mean active muscle stiffness results (mTRs and mTRe), CC1 results (mTRe).

It is unclear where levels of active muscle stiffness and co-contraction observed during the triad exercises should fit with respect to the levels observed during section G96 of the Ann Southam task and the C major scale. In fact, it is difficult to compare the data from the scale and Ann Southam piece with data from the triad exercises. In the scale and Ann Southam piece, both hands play at the same time, and only one note per hand is depressed at a time. In the triad exercises, multiple keys are depressed at the same in only the right hand.

Although both the scale and Ann Southam piece are tasks where the left and right hands play together, and only one finger per hand plays at a time, the hand positions

\footnotetext{
${ }^{2}$ No statistical test was used to make comparisons between experimental tasks. Although differences in mean active muscle stiffness and co-contraction may be observed, these differences may not be significant.
} 
assumed during each task are quite different. While playing the scale, the right hand travels up the keyboard, approximately $65 \mathrm{~cm}$ to the right, then down the keyboard, $65 \mathrm{~cm}$ to the left, moving across the midline of the body in both directions. In the Ann Southam piece, the hands remain positioned over the same keys for the entire exercise. The ellipse that represents stiffness at the hand (e.g. [22]) changes based on the position of the arm. Since active muscle stiffness and co-contraction affect joint stiffness, it is reasonable to assume that differences in active muscle stiffness and co-contraction between the scale and Ann Southam piece may be a result of the different positions of the hand with respect to the keyboard.

Based on the summary plots shown in Figures 8.1, 8.2, 8.3, 8.4, 8.5, 8.6, 8.7, 8.8 and 8.9, there appears to be no broad pattern that characterizes the data. This was somewhat expected because all three playing tasks are quite different in nature, and are subject to differences in playing techniques used by participants.

\subsection{Discussion of All Tasks}

Co-contraction has been expressed as a ratio [17,30-35], the area of overlap of linear envelopes [17, 30, 38-40], and the PPM [36], and co-contraction has been measured during a variety of tasks:

- Static tasks, such as isometric knee flexion and extension exercises [30];

- Dynamic tasks that are cyclical (and repetitive) in nature, such as stationary cycling [61], walking [30], freestyle swim stroke [41]; and

- Dynamic tasks that are not as predictable in nature, such as piano playing [37].

The primary goal of this study was to detect if a significant and measurable change in co-contraction, as measured by EMG, took place during a prescribed set of piano 
playing tasks. The study was successful in that, for the most part, significant changes in active muscle stiffness and co-contraction occurred as predicted.

As with any task quantifying co-contraction under dynamic conditions, the results need to be interpreted with some caution. Values of active muscle stiffness and co-contraction provide estimates only; interpretations of EMG measurements (e.g. co-contraction) take into account a set of physiologic assumptions that may not be justified [30]. The MES measured by EMG equipment is affected by various factors such as tissue composition. Thus being dehydrated can affect measured EMG levels [62]. EMG equipment must be used properly: if electrodes are not placed properly - they should be placed over the belly of the muscle, away from the neuromuscular junction, the muscle-tendon interface and the edges of the muscle - they will yield smaller signals. Also, because the MES is not a direct measure of force production, the MES needs to be normalized.

\subsubsection{Normalization}

There are multiple ways to normalize EMG results. In this study, a number of isometric contractions were performed to elicit high levels of activity in forearm muscles. Multiple exercises were chosen because it was unknown what isometric contraction would successfully target all four extrinsic hand muscles, two having their primary action at the wrist, and two having their primary action at the phalanges. In his article on "Standards for Reporting EMG Data" Merletti [63] recommends that the author of a study utilizing EMG report how subjects were trained to perform the MVC, specifically the rate of rise of the force, the velocity of shortening or lengthening, the range of joint angle and muscle length in non-isometric contractions, and the load applied during a non-isometric contraction. In published studies, these guidelines are not always followed. In our study, four isometric contractions were used to 
determine the value of the MVC. The RMS-processed signals of the FCU, ECU, FDS and EDC obtained from the four MVC manoeuvres were averaged over three-second intervals, and the highest value observed in each muscle was used to normalize EMG measurements of that muscle.

This is not the only procedure to elicit an MVC. EMG measurements can be scaled to the maximum or mean value observed during the task being studied, or scaled to the maximum or mean of an isometric contraction intended to elicit an MVC [30]. For instance, in a study by Unnithan et al. [38], the MVC used by researchers was the largest value of activity measured in each muscle during the MVC manoeuvre. Where a greater activity level was observed during an experimental task (e.g. walking on a treadmill at $90 \%$ fastest walking speed) this was used as the MVC, rather than the value from the MVC manoeuvre.

In order to obtain the best MVC estimate, it is important that subjects be properly trained on how to perform the MVC exercise(s). If they are not, is it possible for the MVC values to be 20-30\% less than they would be with proper training [63].

In some settings, reporting normalized EMG measurements has its limitations. Where people suffer from spasticity or have muscular problems that prevent them from producing a consistent contraction, calculating an MVC value using an isometric contraction might underestimate the value of the MVC, thus distorting the results $[30]$.

\subsection{Evaluation of Study Design}

Hypotheses SC-LO and TR-HI proposed that, during the $\mathrm{C}$ major scale and triad exercises, levels of co-contraction would be low and high, respectively. It was not entirely possible to confirm or reject these hypotheses because the literature reviewed 
by the author did not address what qualifies as low and high co-contraction.

Additionally, the MVC manoeuvres chosen for our study did not elicit accurate MVC values. This was revealed by plots of active muscle stiffness versus time (refer to Appendices $\mathrm{H}, \mathrm{K}$ and $\mathrm{O}$ ) and co-contraction versus time (refer to Appendices $\mathrm{H}$, $\mathrm{L}$ and $\mathrm{P}$ ), where the linear envelopes representing active muscle stiffness and cocontraction occasionally exceeded $100 \%$ MVC, which is physiologically impossible. Determination of MVC values could be improved by employing a dynamometer to measure the force or torque generated by the FCU, ECU, FDS and EDC during MVC manoeuvres. Alternatively, the same approach as Unnithan et al. [38] could be used: use the greatest activation observed in the study as the MVC value, whether that value was obtained from an isometric contraction intended to elicit the MVC or the dynamic task being studied.

\subsubsection{Measurements}

In Chapter 6 it was noted that participants experienced increases in discomfort while playing the triad exercises, but this increase in discomfort was not mirrored by an increase in mean active muscle stiffness and co-contraction. There were significant increases in mean active muscle stiffness and co-contraction in approximately $50 \%$ of cases, contrary to hypothesis TR-SC, which proposed that significant increases in mean active muscle stiffness and co-contraction would occur in all cases. It was suggested in Section 6.2.2 that the MES be measured from additional muscles controlling the wrist and fingers to observe if they underwent increases in active muscle stiffness and co-contraction. It is possible that these other muscles contributed to the production of the required movements [19]. This recommendation could be applied across all three playing tasks to understand which muscles contributed to movements and which ones did not. All three playing tasks evaluated in this study incorporated the 
use of the thumb; however, no muscles that control thumb movements were measured.

In addition to EMG measurements, reflective markers could have been placed on joints, allowing joint position to be accurately captured and analysed. Analysis of joint position could help clarify the relationship between hand positions on the keyboard and levels of active muscle stiffness and co-contraction.

\subsubsection{Co-contraction Measurement}

The measure of co-contraction used in our study provides a benefit over using a ratio of agonist compared to antagonist activity. When a ratio is used as an indicator of co-contraction, this ratio should be interpreted in the context of the task. Also, it is useful to have another measure of co-contraction that indicates magnitude, as was done in Damiano et al.'s study [30]. Using the geometric mean to compute co-contraction is beneficial because small magnitudes of co-contraction are indicated by smaller numbers; likewise, co-contractions of large magnitudes are indicated with larger numbers. The geometric mean offers the advantage over the arithmetic mean as it does not skew the value of the mean towards the larger number. Thus, for co-contraction to be large, both values of active muscle stiffness must be large.

The major flaw in our measures of co-contraction is that contributions from other muscles with primary and secondary actions at the wrist are not taken into account. This is not a criticism of our calculation of co-contraction; it is a criticism of the

methods used to capture co-contraction data. There are 15 muscles with primary and secondary actions at the wrist: nine wrist extensors and six wrist flexors [9]. Although wrist stiffness is partially determined by the co-contraction of muscles around it, activities of four muscles may not fully signal changes in stiffness at the wrist. 


\section{Chapter 9}

\section{Conclusions, Contributions and}

\section{Recommendations}

This study was designed to assess changes in active muscle stiffness and cocontraction during a variety of piano playing tasks using EMG. To date, there has only been one other study that has used EMG to evaluate changes in co-contraction while playing the piano [37]. EMG successfully detected where there were significant increases, decreases or no significant changes in active muscle stiffness and cocontraction.

\subsection{Experimental Study Conclusions}

The first task was to play a four-octave $\mathrm{C}$ major scale, ascending and descending, with both hands in parallel motion, three times continuously. Since most advanced pianists are quite familiar with the $\mathrm{C}$ major scale, it was hypothesized (hypothesis SC-LO) that active muscle stiffness and co-contraction would be low during the scale task; however, this was not the case. It was also hypothesized (hypothesis SC-NC) that active muscle stiffness and co-contraction would not change significantly from the beginning to the end of the scale. In most cases, mean active muscle stiffness and 
co-contraction of the ascending segments of the $\mathrm{C}$ major scale were not significantly different from each other. The same was found to be true of the descending segments of the $\mathrm{C}$ major scale. It was speculated that high levels of active muscle stiffness and co-contraction are likely due to a high note rate; the scale had the highest note rate of any of the exercises examined in our study.

1. Conclusion SC-LO: Active muscle stiffness and co-contraction levels were not low while playing the $\mathrm{C}$ major scale.

2. Conclusion SC-NC: Active muscle stiffness and co-contraction levels did not change significantly from the beginning to the end of the $\mathrm{C}$ major scale.

The second task was to play two triad exercises. These exercises are also described as finger independence exercises. It was hypothesized (hypothesis TR-HI) that levels of active muscle stiffness and co-contraction would be high. In most cases, it was found that levels of mean active muscle stiffness and co-contraction were greater than 15\% MVC at the beginning (segment 2) and end (segment 4) of both triad exercises. It was hypothesized (hypothesis TR-SC) that there would be significant increases in active muscle stiffness and co-contraction from the start to the end of the exercise. This occurred in approximately $50 \%$ of cases. This is possibly because levels of muscle activity were high at the start of the exercise, and an increase in the activity levels of the FCU, ECU, FDS and EDC may have been restricted by reciprocal inhibition. Another possibility is that the anatomical positions assumed in TR1 and TR2 determined the maximum force these muscles could produce, and the maximum force was reached as soon as the participants began to play the alternating notes of TR1 and TR2.

3. Conclusion TR-SC: Mean active muscle stiffness and co-contraction significantly increased from the start to the end of TR1 and TR2, but only in $50 \%$ of 
cases. Further investigation is required as participants in this study used two distinct strategies (i.e. holding and tapping the alternating notes of TR1 and TR2) to execute the exercise.

4. Conclusion TR-HI: Active muscle stiffness and co-contraction were high throughout the durations of the exercises.

It was observed that participants experienced more discomfort when executing TR1 than TR2, and that some participants tapped the alternating notes in the triad exercises rather than holding them. This led to the development of two additional hypotheses: significant increases in mean active muscle stiffness and co-contraction will be more frequently observed in TR1 than in TR2 (hypothesis TR-N1), and where participants tapped the alternating notes, rather than holding them, no significant changes in mean active muscle stiffness and co-contraction will be observed (hypothesis TR-N2). Neither hypothesis could be confirmed as there were not enough participants to identify any patterns.

5. Conclusion TR-N1: Significant increases in mean active muscle stiffness and co-contraction were not observed more frequently in TR1 than in TR2, although this may be attributed to the elimination of results of participants who did not execute the task as required.

6. Conclusion TR-N2: It is unclear what effect tapping, rather than holding, the alternating notes in TR1 and TR2 had on significant changes in mean active muscle stiffness and co-contraction. There were not enough participants to establish any discernible patterns.

The third and final task was to play a piece composed by Ann Southam. This piece was originally composed for a study by Vant examining the effects of force 
perturbations on driving point impedance while playing the piano, specifically the Ann Southam piece. It was hypothesized (hypothesis AS-SC) that there would be a significant increase in active muscle stiffness and co-contraction from the beginning of the piece to the end of section G96, where note rate and loudness are highest. Significant increases in mean active muscle stiffness and co-contraction were observed from sections A to B, A to C96, A to G96 and C96 to G96. These findings were contrary to the outcome of Vant's analysis, which found no significant changes in displacement between sections A, B and C96 when a force perturbation was applied to the wrist. (Vant was unable to analyze section G96.) It was also hypothesized (hypothesis AS-BE) that there would be significant differences in active muscle stiffness and co-contraction from the beginning of the piece to the end (which is the same as the first four bars of the piece, plus one note). Comparing sections A to A' and B to B', there are few cases where significant changes in mean active muscle stiffness and co-contraction were observed. This refutes the idea that because sections A' and B' are preceded by a passage that is played loudly and has a high note rate, sections A' and B' would have significantly higher mean active stiffnesses and co-contraction than sections $A$ and $B$, respectively. Whether these findings can be translated to actual performance conditions is unclear; the composition by Ann Southam is not a long piece of music, and it does not accurately reflect playing duration of advanced pianists.

7. Conclusion AS-SC: Active muscle stiffness and co-contraction significantly increased from the beginning of the Ann Southam piece to the end of bar 20 . In most cases, significant increases in active muscle stiffness and co-contraction were observed from A to B and from A to C96, thus contradicting Vant's findings. 
8. Conclusion AS-BE: Active muscle stiffness and co-contraction were not significantly different between the beginning (sections $\mathrm{A}$ and $\mathrm{B}$ ) and end (sections $A^{\prime}$ and B') of the Ann Southam piece, but were significantly different between $\mathrm{W}$ and $\mathrm{W}^{\prime}$.

\subsection{Contributions}

The findings of this study have set the framework for future studies. We were able to identify several variables, such as note rate, loudness, arm position and playing duration, which may affect levels of active muscle stiffness and co-contraction. It is highly suspected that increased note rate is accompanied by an increase in active muscle stiffness and co-contraction, but the results are not conclusive. It would be worthwhile to consider the effect of the identified variables in future studies.

This study was able to show that perceived stiffness is not always reflected in significant increases of active muscle stiffness and co-contraction: where participants perceived an uncomfortable increase in stiffness at the wrist, a significant increase in active muscle stiffness and co-contraction occurred in only $50 \%$ of cases.

\subsection{Recommendations for Future Study}

Further study is needed to tease apart the relationship between note rate, loudness, arm position and duration with active muscle stiffness and co-contraction.

Future studies examining the effect of these variables on active muscle stiffness and co-contraction in scales could be assessed using:

- Scales played at different tempos (e.g. with a note rate ranging from 1 note per second to 8 notes per second), 
- Scales played at different loudness levels (e.g. pianissimo, piano, mezzo piano, mezzo forte, forte and fortissimo),

- Different lengths of scale (e.g. two-octave scale compared to four-octave scales),

- Scales played for different lengths of time (e.g. three four-octave scales, ascending and descending, played continuously - approximately $23.3 \mathrm{~s}$ - compared with 38 four-octave scales, ascending and descending, played continuous - approximately 5 minutes).

The Ann Southam piece could be used to examine the effect of these four variables on active muscle stiffness and co-contraction by having participants play:

- The Ann Southam piece with no dynamics,

- Section B (or C) of the Ann Southam piece repeated several times, but louder each time,

- The Ann Southam piece read up or down an octave or two while maintaining the same sitting position,

- The Ann Southam piece played once - approximately 0:02:36 - compared with playing it four times continuously - approximately $0: 10: 24$.

It is more difficult to assess the effect of note rate, loudness, arm position and duration on active muscle stiffness and co-contraction while playing the triad exercises. Instead, the effect of tapping the alternating notes, compared to holding them, on mean active muscle stiffness and co-contraction should be examined. 


\section{List of References}

[1] M. Redmond and A. M. Tieman, "Knowledge and practices of piano teachers in preventing playing-related injuries in high school students," Medical Problems of Performıng Artısts, vol. 16, no. 1, p. 32-38, 2001.

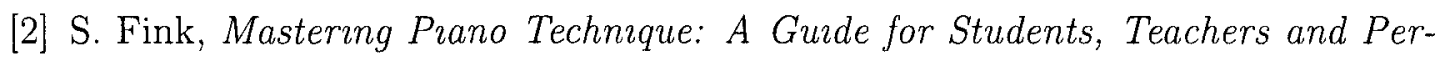
formers. Oregon: Amadeus Press, 1992.

[3] C. Vant, "Driving point impedance measurement during piano playing," Master's thesis, Carleton University, 2007.

[4] D. L. Russell, "Establishing a biomechanical basis for injury preventative piano pedagogy," Revue de recherche en éducation musicale, vol. 24, p. 105-117, 2006.

[5] T. Mark, What Every Pianıst Needs to Know About the Body. Chicago: GIA Publications, 2004.

[6] "Video 1: Introductory principles and concepts." The Taubman Institute of Piano, NY, 1995.

[7] A. Fraser, The Craft of Pıano Playıng. The Scarecrow Press Inc., 2003.

[8] A. Grieco, E. Occhipinti, D. Colombini, O. Menoni, M. Bulgheroni, C. Frigo, and S. Boccardi, "Muscular effort and musculo-skeletal disorders in piano students: electromyographic, clinicial and preventative aspects," Ergonomıcs, vol. 32, p. $697-716,1989$.

[9] N. M. Austin, Joint Structure 8 Function: A Comprehensive Analysıs, ch. The wrist and hand complex, p. 321-336. Philadelphia: F.A. Davis Company, 4 ed., 2005.

[10] F. H. Martini, M. J. Timmons, and R. B. Tallitsch, Human Anatomy. Upper Saddle River, NJ: Pearson Education Inc., 4 ed., 2003. 
[11] C. R. Ethier and C. A. Simmons, Introductory bıomechanıcs: from cells to organısms, p. 335. Cambridge, NY: Cambridge University Press, 2007.

[12] "Chapter 8. Control of movement." http://behavioralphys.wikispaces.com /Chapter+8, 2010. Retrieved on January 20, 2011.

[13] L. Peachey, "The sarcoplasmic reticulum and transverse tubules of the frog's sartorius," The Journal of Cell Brology, vol. 25, no. 3, p. 209-231, 1965.

[14] E. Marieb, Human Anatomy and Physiology. Redwood City, CA: Benjamin/Cummings, 3 ed., 1995.

[15] M. Nordin and V. Frankel, eds., Basıc Bromechanıcs of the Musculoskeletal System. Malvern, PA: Lea \& Febiger, 2 ed., 1989.

[16] C. De Luca, Encyclopedıa of Medical Devices and Instrumentation, ch. Electromyography, p. 98-109. Wiley-Interscience, 2006.

[17] D. Winter, Bromechanıcs and motor control of human movement. Hoboken, NJ: Wiley, 4 ed., 2009.

[18] T. McMahon, Muscles, reflexes, and locomotıon. Princeton, NJ: Princeton University Press, 1984.

[19] R. Osu and H. Gomi, "Multijoint muscle regulation mechanisms examined by measured human arm stiffness and EMG signals," Journal of Neurophysiology, vol. 81, no. 4, p. 1458-1468, 1999.

[20] T. Flash and F. Mussa-Ivaldi, "Human arm stiffness characteristics during the maintenance of posture," Experımental Brain Research, vol. 82, p. 315-326, 1990.

[21] P. Morasso, "Spatial control of arm movements," Experımental Brain Research, vol. 42, p. 223-227, 1981.

[22] F. Mussa-Ivaldi, N. Hogan, and E. Bizzi, "Neural, mechanical, and geometric factors subserving arm posture in humans," Journal of Neuroscuence, vol. 5, no. 10 , p. 2732-2743, 1985.

[23] T. Tsuji, P. Morasso, K. Goto, and K. Ito, "Human hand impedance characteristics during maintained posture," Brologıcal Cybernetıcs, vol. 72, no. 6, p. 475-485, 1995. 
[24] H. Gomi and M. Kawato, "Human arm stiffness and equilibrum-point trajectory during multı-joint movement," Brologıcal Cybernetıcs, vol. 76, p. 163-171, 1997.

[25] D. Shin, J. Kim, and Y. Koike, "A myokinetic arm model for estimating joint torque and stiffness from EMG signals during maintained posture," Journal of Neurophysiology, vol. 101, no. 1, p 387-401, 2009.

[26] R. Osu, D. Franklin, H. Kato, H. Gomı, K. Domen, T. Yoshioka, and M. Kawato, "Short- and long-term changes in joint co-contraction associated with motor learning as revealed from surface EMG," Journal of Neurophysiology, vol. 88, p. 991-1004, 2002

[27] P. Gribble, L. Mullin, N. Cothros, and A. Mattar, "Role of cocontraction in arm movement accuracy," Journal of Neurophysiology, vol. 89, p 2396-2405, 2003.

[28] D. Damiano, "Reviewing muscle cocontraction: Is it a developmental, pathological, or motor control issue?," Physical and Occupational Therapy in Pedratrics, vol. 12 , no. 4, p. 3-20, 1993.

[29] P. Gribble and D. Ostry, "Independent coactivation of shoulder and elbow muscles," Experımental Brain Research, vol. 123, no 3, p. 355-360, 1998.

[30] D. Damiano, T. Martellotta, D. Sullivan, K. Granata, and M. Abel, "Muscle force production and functional performance in spastic cerebral palsy: Relationship of cocontraction," Archives of Physical Medicine and Rehabulitation, vol. 81, no. 7, p. $895-900,2000$.

[31] B. Myklebust, G. Gottlieb, and G. Agarwal, "Stretch reflexes of the normal infant," Developmental Medıcıne and Child Neurology, vol. 28, no. 4, p. 440-449, 1986.

[32] M. Levin and C. Hui-Chan, "Ankle spasticity is inversely correlated with antagonist voluntary contraction in hemiparetic subjects," Electromyography and Clinıcal Neurophysıology, vol. 34, no. 7, p. 415-425, 1994.

[33] A. Ikeda, M. Abel, K. Granata, and D. Damiano, "Quantification of cocontraction in spastic cerebral palsy," Electromyography and Clinıcal Neurophysıology, vol. 38, no. 8, p. 497-504, 1998.

[34] M. Busse, C. Wiles, and R. van Deursen, "Co-activation: 1ts association with weakness and specific neurological pathology," Journal of Neuroengineering and Rehabulitation, vol 3, no. 26, p. published online, 2006. 
[35] J. Chae, B. Yang, G. Park, and I. Labatia, "Muscle weakness and cocontraction in upper limb hemiparesis: relationship to motor impairment and physical disability," Neurorehabulıtation and Neural Repair, vol. 16, no. 3, p. 241-248, 2002.

[36] M. Cowan, D. Stilling, S. Naumann, and G. Colborne, "Quantification of antagonist muscle coactivation in children with spastic diplegia," Clinıcal Anatomy, vol. 11 , no. 5, p. 314-319, 1998.

[37] M. Yoshie, K. Kudo, T. Murakoshi, and T. Ohtsuki, "Music performance anxiety in skilled pianists: effects of social-evaluative performance situation on subjective, autonomic, and electromyographic reactions," Experimental Brain Research, vol. 199, p. 117-126, 2009.

[38] V. Unnithan, J. Dowling, G. Frost, and O. Bar-Or, "Role of cocontraction in the $\mathrm{O}_{2}$ cost of walking in children with cerebral palsy," Medrcine 8 Scrence in Sports \& Exercise, vol. 28, no. 12, p. 1498-1504, 1996.

[39] A. Lamontagne, C. Richards, and F. Malouin, "Coactivation during gait as an adaptive behavior after stroke," Journal of Electromyography and Kinesıology, vol. 10 , no. 6 , p. 407-415, 2000.

[40] K. Thoroughman and R. Shadmehr, "Electromyographic correlates of learning an internal model of reaching movements," Journal of Neuroscience, vol. 19, no. 19, p. $8573-8588,1999$.

[41] V. Caty, Y. Aujouannet, F. Hintzy, M. Bonifazi, J. Clarys, and A. Rouard, "Wrist stabilization and forearm muscle coactivation during freestyle swimming," Journal of Electromyography and Kinesıology, vol. 17, p. 285-291, 2007.

[42] T. Heiden, D. Lloyd, and T. Ackland, "Knee joint kinematics and muscle cocontraction in knee osteoarthritis patient gait," Clınıcal Bıomechanıcs, vol. 24, no. 10, p. $833-841,2009$.

[43] M. Yeadon, M. King, S. Forrester, G. Caldwell, and M. Pain, "The need for muscle co-contraction prior to a landing," Journal of Bromechanıcs, vol. 43, no. 2, p. 364-369, 2010.

[44] Y. Liu and X. $\mathrm{J}_{1}$, "Research on the co-contraction of shoulder and upper limb muscles in driver's steering manoeuvre," pp. 5781-5784, 2010. 
[45] D. Robertson, "Principles of EMG: Recording." Coursenotes APA 4311 University of Ottawa http://www.health.uottawa.ca/biomech/courses/apa4311/emgp1.pdf, 2006. Retrieved on May 17, 2011.

[46] Delsys, "Surface EMG sensors specifications." http://www.delsys. com/Products/EMGSensors_Specifications.html, 2008. Retrieved on May $19,2011$.

[47] C. DeLuca, "A practicum on the use of sEMG signals in movement sciences." http://www.delsys.com/KnowledgeCenter/Practicum.html, 2008. Retrieved on May 17, 2011.

[48] E. F. Delagi, A. Perotto, J. Iazzetti, and D. Morrison, Anatomic Gunde for the Electromyographer, ch. The Limbs, p. 36-53. Springfield, IL: Charles C. Thomas., 2 ed., 1980.

[49] G. Shan and P. Visentin, "Utility of EMG to quantify activity levels in small muscles (Letters to the editor)," Medical Problems of Performing Artısts, vol. 20, no. 2, p. 111-112, 2005.

[50] P. Ludewig and J. Borstead, Jont Structure \& Function: A Comprehensive Analysıs, ch. Shoulder Joint, p. 265. Philadelphia: F.A. Davis Company, 4 ed., 2005.

[51] E. Dohnányi, Essentıal Finger Exercıses for Obtaınıng a Sure Pıano Technıque. Budapest: Editio Musica, 1950.

[52] T. Eerola and P. Toivianinen, MIDI Toolbox: MATLAB Tools for Musıc Research. Department of Music, University of Jyväskylä, Kopijyvä, Jyväskylä, Finland, 2004.

[53] M. Prewett, "HexEdit v1.03 software." http://www.physics.ohiostate.edu/ ${ }^{2}$ prewett/hexedit/, 1993-2008. Retrieved on May 17, 2011.

[54] K. Revis, "SysEx Librarian software." http://www.snoize.com/SysExLibrarian/, 2002-2008. Retrieved on May 17, 2011.

[55] B. Wristen, "Avoiding piano-related injury: A proposed theoretical procedure for biomechanical analysis of piano technique," Medical Problems of Performing Artusts, vol. 15, p. 55-64, 2000. 
[56] J. McDonald, Handbook of Brological Statıstıcs. Baltimore, MD: Sparky House Publishing, 2 ed., 2009.

[57] B. Day, C. Marsden, J. Obeso, and J. Rothwell, "Reciprocal inhibition between the muscles of the human forearm," Journal of Physiology, vol. 349, p. 519-534, 1984.

[58] Tanager AudioWorks, Inc., "Chirp virtual MIDI keyboard controller user guide (build 1.2)." http://www.tanageraudioworks.com/Datasheets/ Chirp_Virtual_MIDI_Keyboard_Controller_User_Guide.pdf, 2008. Retrieved on May 17, 2011.

[59] B. Jonsson, "The static load component in muscle work," European Journal of Applied Physıology, vol. 57, p. 305-310, 1988.

[60] T Ostensvik, K. Veiersted, and P. Nilsen, "A method to quantify frequency and duration of sustained low-level muscle activity as a risk factor for musculoskeletal discomfort," Journal of Electromyography and Kinesiology, vol. 9, p. 283-294, 2009.

[61] T. Johnston, A. Barr, and S. Lee, "Biomechanics of submaximal recumbent cycling in adolescents with and without cerebral palsy," Physical Therapy, vol. 87, no. 5, p. 572-585, 2007.

[62] J. Basmajian and C. DeLuca, Muscles alvve: Therr functions revealed by electromyography. Baltimore, MD: Williams \& Wilkins, 1985.

[63] R. Merletti, "Standards for reporting EMG data." http://www.isekonline.org/standards_emg.html, 1999. Retrieved on May 17, 2011.

[64] C. Corby, "The human body." http://www emergencymedicaled.com/224 The\%20Human\%20Body.htm, 2002-2009. Retrieved on May 17, 2011 


\section{Appendix A}

\section{Anatomical Terminology}

When describing human anatomical orientation and displacement, rather than

using an $(\mathrm{x}, \mathrm{y}, \mathrm{z})$ coordinate system, everything is described with reference to the anatomical position, illustrated in Figure A.1. There are many terms that are used to describe direction; however, six directional terms are relevant here:

Medial: towards the midline of the body (e.g. in the anatomical position, the fifth finger is medial to the thumb);

Lateral: away from the midline of the body (e.g. in the anatomical position, the thumb is lateral to the fifth finger);

Proximal: closer to the point of attachment of a limb to the trunk of the body (e.g. the elbow is proximal to the wrist);

Distal: further from the point of attachment of a limb to the trunk of the body (e.g. the ankle is distal to the knee);

Ventral: at or near the front of the body; when describing the surfaces of the hand the term palmar is used; 


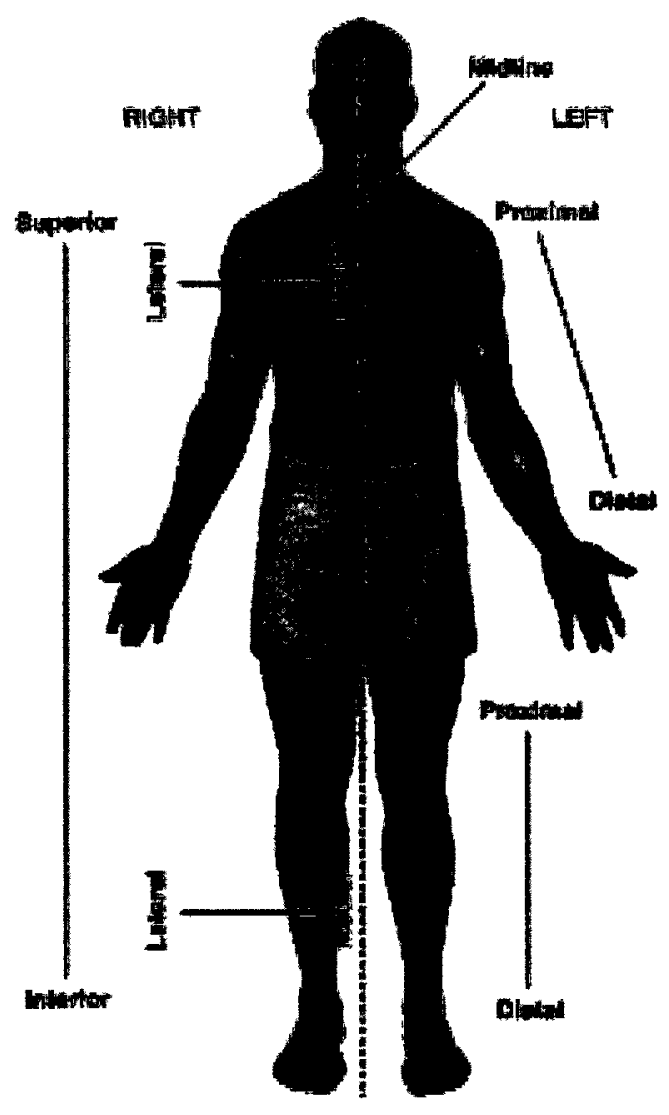

Figure A.1: Front view of a person in the anatomical position. Obtained from [64]. 
Dorsal: at or near the back of the body; when describing the surfaces of the hand the term volar is used.

There are also anatomical terms for describing the direction of displacement or movement of a body segment. Because parts of the body are capable of rotary and translatory motions, terms of movement reference the movements of the body segments were they in the anatomical position, even if the body is not positioned so, making the terms of movement independent of viewer perspective. Here, several terms relevant to the movements of the human forearm are defined:

Flexion: Rotation of two body segments in the same plane about a joint so that the ventral surfaces are brought closer together (e.g. bringing the wrist towards the shoulder results in elbow flexion);

Extension: Opposite of flexion; rotation of two body segments in the same plane about a joint so that the dorsal surfaces are brought closer together (e.g. during the heel-on portion of the gait cycle, the knee is extended);

Abduction: Rotation of a body segment about a joint so that the body segment moves away from the midline;

Adduction: Opposite of abduction; rotation of a body segment about a joint so that the body segment moves toward the midline (e.g. abduction and adduction of the hip joint occur when doing jumping jacks) ${ }^{1}$;

Pronation: Rotation of the forearm or foot turning the palmar surfaces to face posteriorly or down, respectively;

\footnotetext{
${ }^{1}$ Abduction and adduction of the wrist are often referred to as deviation. Deviation in the direction of the radius, or radial deviation, is equivalent to abduction; deviation in the direction of the ulna, or ulnar deviation, is equivalent to adduction.
} 
Supination: Opposite of pronation; rotation of the forearm or foot turning the palmar surfaces to face anteriorly or up, respectively (e.g cupping one's hands as in begging for soup involves supination of the forearms). 


\section{Appendix B \\ Letter of Information, Consent Form and Questionnaire for Participants}




\section{Carleton \\ U N I ER S I T}

Canada's Capital University

\section{Letter of Information}

Dear Participant,

A significant number of musicians experience playing related injuries despite many years of careful training. Many researchers suggest that these injuries are a result of the technique that each musician uses to play. This collaborative research project is investigating possible relationships between piano technique and patterns of muscle activation during piano playing. We are working to develop approaches that will dramatically reduce the occurrences of these injuries. This exciting research includes contributions from researchers in the field of biomechanics at Carleton University (Caroline Andison and Prof. Donald L. Russell) and the University of Ottawa's Piano Pedagogy Research Laboratory. We are currently recruiting subjects to participate in our project and are requesting your aid. Below, you will find a description of the study and a description of what your participation in the experiment would involve. Should you wish to volunteer to take part in this groundbreaking study, or if you would like more information, please email Caroline Andison at candison@connect.carleton.ca.

Research Project: EMG-based assessment of co-contraction in muscles crossing the wrist during piano playing.

\section{Target group:}

We are seeking participants 18 years of age or older and who are undergraduate music students majoring in piano or pianists who have been playing the piano for at least 15 years.

\section{Objective of the study:}

The objective of the study is to examine the contribution of different groups of muscles in the hand and wrist during piano playing using surface electromyography (EMG). The results will be used to determine if there is an increase (or change) in co-contraction while playing the prescribed exercises.

Where the studies will take place:

The experiments will take place at the University of Ottawa's Piano Pedagogy Research Laboratory and the address is listed below:

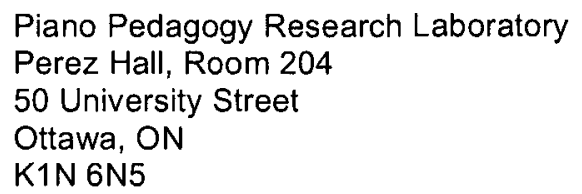

The newly constructed Piano Pedagogy Research Laboratory is similar to a real piano studio with two intelligent grand pianos, but is enhanced with video and acoustic equipment. These include a variety of video cameras and two large LCD screens. The laboratory is a comfortable area and is child friendly. 


\section{Carleton \\ UN IVERSITY \\ Canada's Capital University}

What is requested of the participants:

Participants will be assigned numbers so that their identities remain anonymous. They will be asked to complete a questionnaire regarding their piano (or other instrument) playing habits. Following the questionnaire, participants will be seated in a comfortable chair and EMG electrodes will be placed on the skin surface of the forearm. These electrodes are small, noninvasive and will be pasted onto the forearm using a non-toxic adhesive. Participants will then be asked to 1) play a $C$ major scale three times, 2) play two exercises involving triads, and 3) sight-read a piece of music composed by Ann Southam that has previously been used for the purpose of measuring tension in the wrist. The EMG data will help us understand how and when forearm muscles are recruited and used during piano performance. Musical instrument digital interface (MIDI) data and video data will also be recorded in order to have auditory and visual records of the participant's performance. The total time commitment for the participant will be 30-45 minutes.

How the participant's privacy will be protected:

Any personal information (name, year of birth, e-mail address, results of questionnaire) collected will only be used within the context of this study. Research data collected will be securely stored under lock and key in the Piano Pedagogy Research Laboratory, and electronic data stored on a computer will be protected by a password. Potentially, these data may be used again in similar studies. Only authorized members of the Piano Pedagogy Research Laboratory, Caroline Andison, Dr. Donald Russell, and students under the supervision of Dr. Russell working on projects of a similar nature will have access to this information. Data will be kept indefinitely.

Risks the participant may face:

There is a small risk of physical discomfort. When the surface electrodes used to collect electromyographic data are removed from the participant's arm, the participant may experience some discomfort. Removal of the surface electrodes will be no more painful than the discomfort experienced when removing an adhesive bandage. There is also a small risk of fatigue due to length of the experiment and the repetitive finger movements involved. However, since the participants will be piano players, it is unlikely that level of fatigue would be greater than that experienced during a typical practice session.

\section{Benefits of the study:}

This study stands to benefit pianists (the participants) and piano teachers. Results of this study will provide pianists with feedback on the level of muscle activity of their own forearms and allow them to alter their technique as they play so to reduce the amount of co-contraction in their muscles crossing the wrist. This feedback can help teachers learn what hand positions and motions cause increased levels of co-contraction, and can help them to better instruct their students. The data collected will help to understand how piansts injure themselves and what changes in technique can be made so that injury does not occur. Given what the participants will gain from this study, the benefits outweigh the risks.

How participant's can withdraw from the study:

Participation in this study is entirely voluntary. Participants may choose to withdraw at any time, whether before or during the experiment, and with or without prior warning. They may also choose not to complete all requested tasks. Should the participant decide to withdraw from the 


\section{Carleton \\ UN I VER S I T Y}

\section{Canada's Capital University}

study before the experiment is complete, we may use the information gathered unless the participant requests that the data be destroyed.

\section{Funding:}

All costs related to this project are paid for by the University of Ottawa and Carleton University.

\section{Compensation:}

The participant will not receive any form of compensation for participating in this study. It is strictly on a voluntary basis.

\section{How to obtain a copy of the results:}

We would be pleased to share the results of this project with you. In order to receive a summary of the results, please contact Donald Russell by email, Donald Russell@,carleton.ca, subject: EMG-piano playing experiment. For any additional information regarding this project, do not hesitate to communicate with us. Our contact information can be found at the bottom of this letter.

\section{Ethical concerns:}

This project was reviewed and received ethics clearance by the Carleton University Research Ethics Committee. If you have any ethical concerns regarding your participation in this study, please contact the Research Ethics Board Chair:

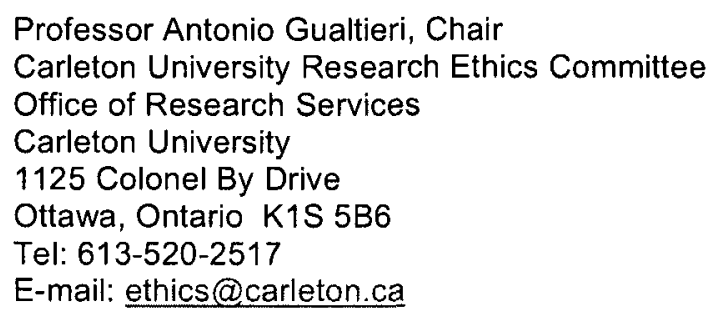

Sincerely,

Caroline Andison, M.A.Sc. candidate Department of Mechanical and Aerospace Engineering

Carleton University

E-mail: candison@connect.carleton.ca
Prof. Donald L. Russell, Ph.D.

Department of Mechanical and Aerospace Engineering Carleton University Phone: (613) 520-5658

E-mail: Donald Russell@carleton.ca

P.S. Please retain a copy of this letter and consent form for your records. 


\section{Consent Form}

\section{EMG-BASED ASSESSMENT OF CO-CONTRACTION IN MUSCLES CROSSING THE WRIST DURING PIANO PLAYING}

Please complete this form and sign it in the presence of the research assistant

I, , confirm that I have read and understood the information presented in the letter of Information about the project

- Yes, I agree to participate in this research project, knowing that I can withdraw at any tıme without cause or reason

\begin{tabular}{|c|c|c|}
\hline 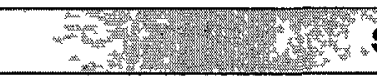 & apleted by th & (2) \\
\hline First name & Last name & \\
\hline Gender Male / Female & Year of bırth & \\
\hline \multicolumn{3}{|l|}{ E-mall (optional) } \\
\hline \multicolumn{2}{|c|}{$\mathrm{Cl}_{\mathrm{C}} \mathrm{C}$} & Date $(\mathrm{dd} / \mathrm{mm} /$ year $)$ \\
\hline \multicolumn{3}{|c|}{ Section to be completed by the Research Assistant } \\
\hline \multicolumn{3}{|l|}{ Research assistant } \\
\hline Signature & & Date $(\mathrm{dd} / \mathrm{mm} /$ year) \\
\hline
\end{tabular}




\section{Carleton \\ U N I ER S I T Y \\ Canada's Capita! University}

\section{Questionnaire}

\section{GENERAL INFORMATION QUESTIONNAIRE}

\section{Participant's number:}

Level of performance reached before university

- grade and institution

(e.g. grade 10 Royal Conservatory)

Last exam passed - grade and institution

Year in undergraduate music program

Participant's age:

Age at which piano studies began:

Frequency of piano practice (days/week): $\begin{array}{ll}1 & 5 \\ 2 & 6 \\ 3 & 7 \\ 4 & \end{array}$

Number of practice sessions per day:

Length of a practice session:

Time of day of practice session(s):

Do you experience any pain while playing? Yes / No 


\section{Appendix C}

\section{Code Listing 1: LoadData algorithm}

This appendix contains the code for the LoadData algorithm used to load EMG and MIDI data into MATLAB. 


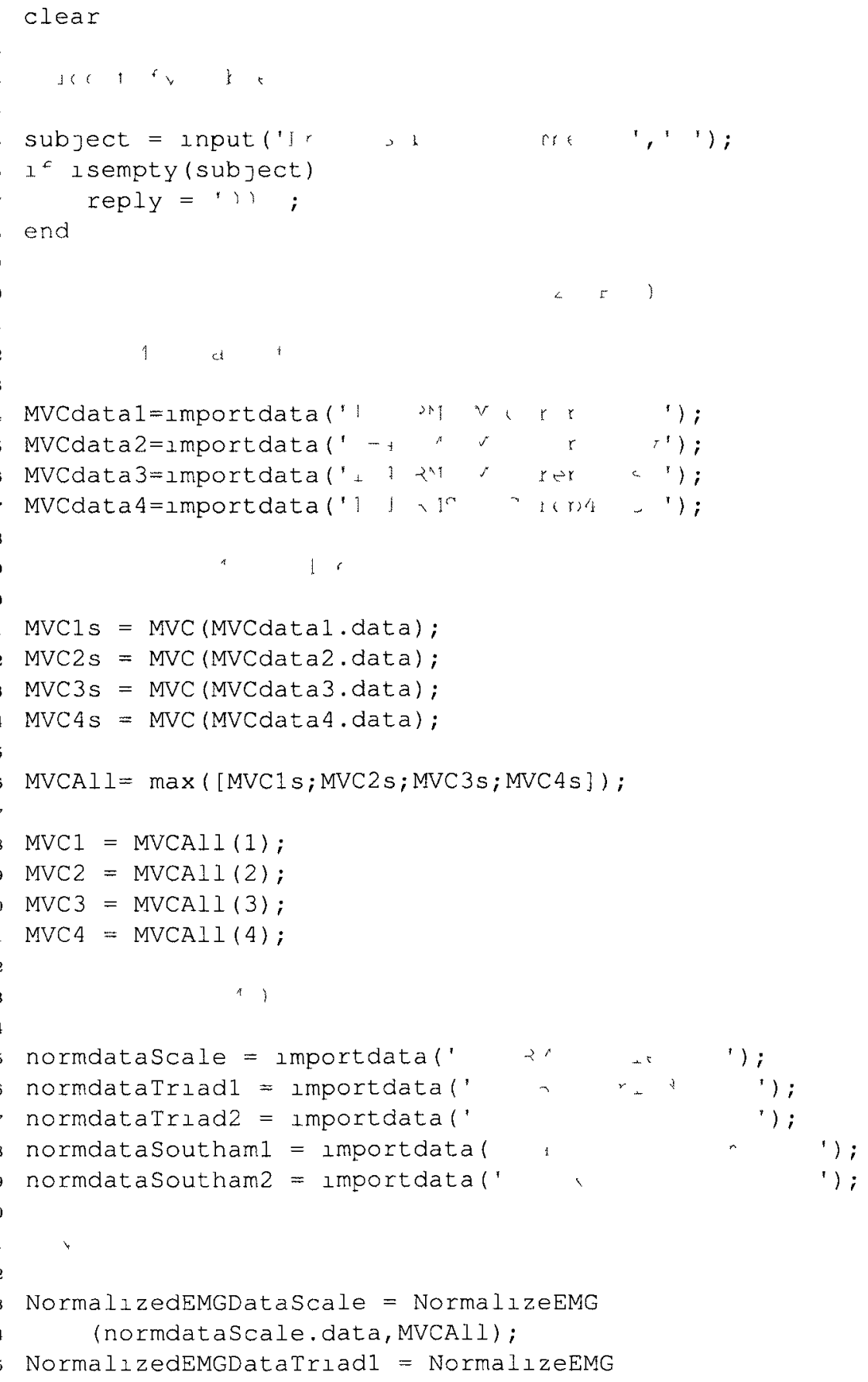




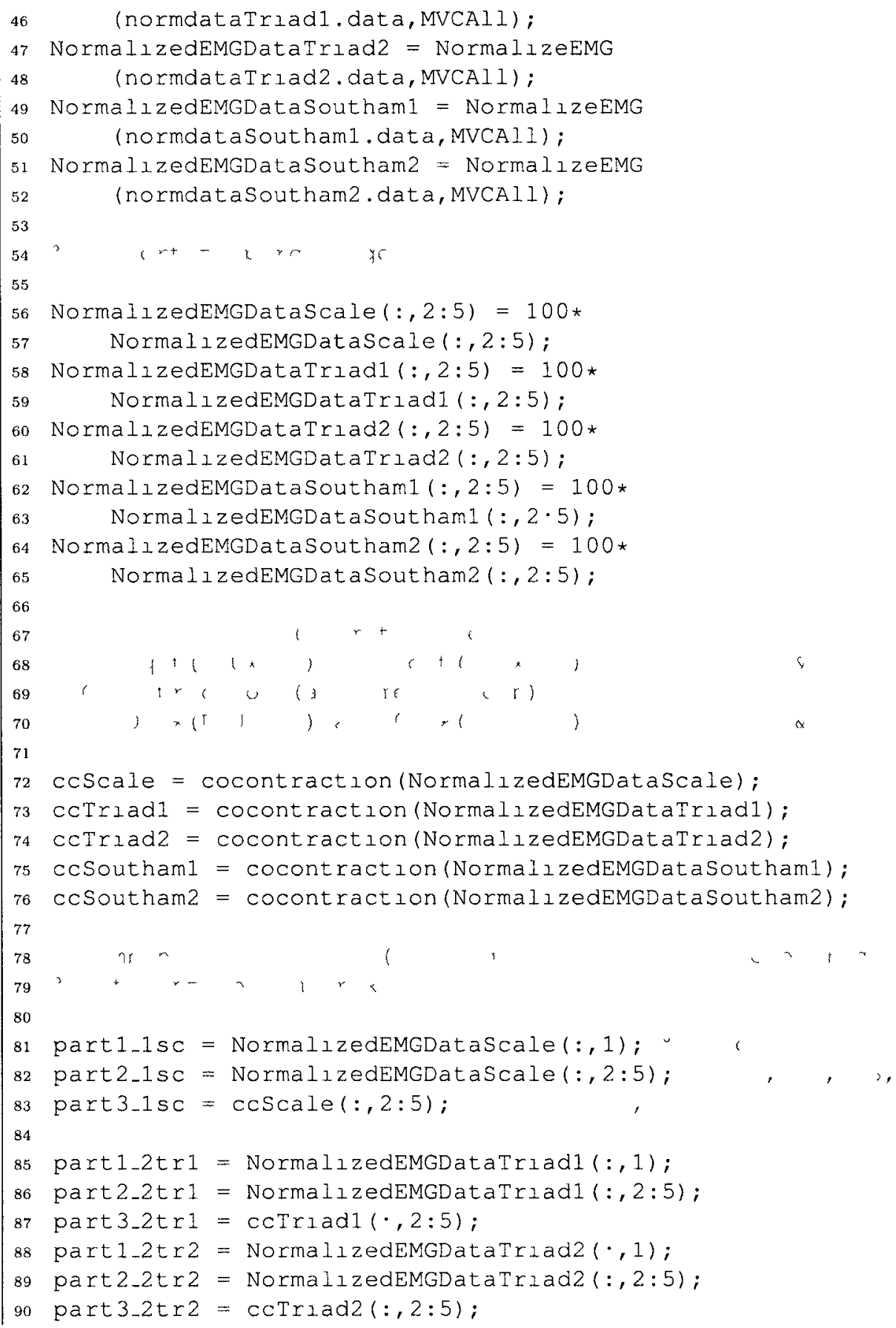


part1_3as 1 = NormalızedEMGDataSoutham $1(:, 1)$

part2-3as $1=$ NormalızedEMGDataSoutham1 $(:, 2: 5)$;

part3_3as $1=$ ccSoutham1 $(:, 2: 5)$;

part1-3as2 = NormalızedEMGDataSoutham2 $(:, 1)$;

part2_3as2 = NormalızedEMGDataSoutham2 $(:, 2: 5)$;

part3_3as2 = ccSoutham2 $(:, 2: 5)$;

EMG_RMS_Scale $=[$ part1_1sc,part2_1sc,part3_1sc];

EMG_RMS_Trlad1 $=[$ part1_2tr1, part2_2tr1, part3_2tr1];

EMG_RMS_Truad2 = [part1_2tr2, part2_2tr2,part3_2tr2];

EMG_RMS_Southam1 = [part1_3as1, part2_3asI,part3_3as1];

EMG_RMS_Southam2 = [part1_3as2, part2_3as2,part3_3as2];

clear part1_1sc part2-1sc part3_1sc

clear part1_2tr1 part2_2tr1 part3_2tr1

clear part1_2tr2 part2_2tr2 part3_2tr2

clear part1_3asl part2_3asl part3_3as 1

clear part1_3as2 part2_3as2 part3_3as 2

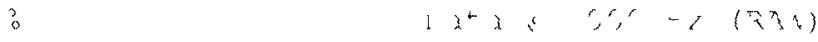

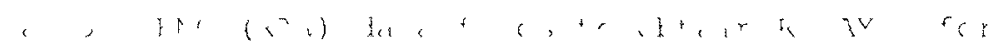

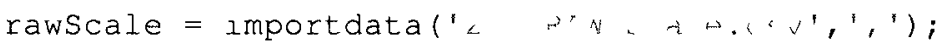

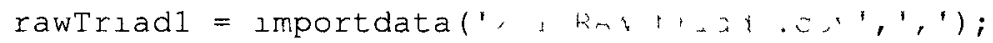

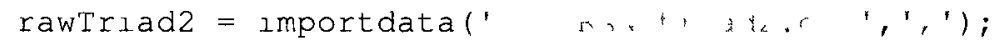

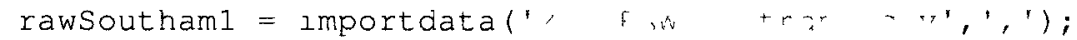

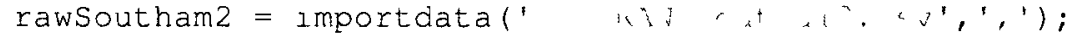

, $\because 1-x^{\prime}$

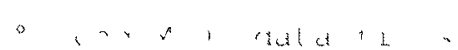

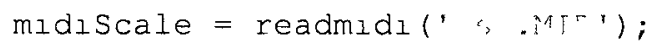

mıdıTrıad1 = readmıdı (", $+, 1 . M, ')$;

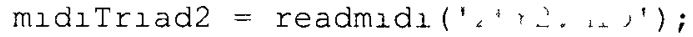

midıSouthaml = readmıdı(", , ");

mıdısoutham $2=\operatorname{readm}\lrcorner d(", . "$.

130

131

132

133

134

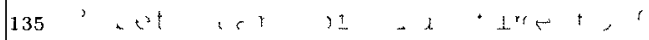




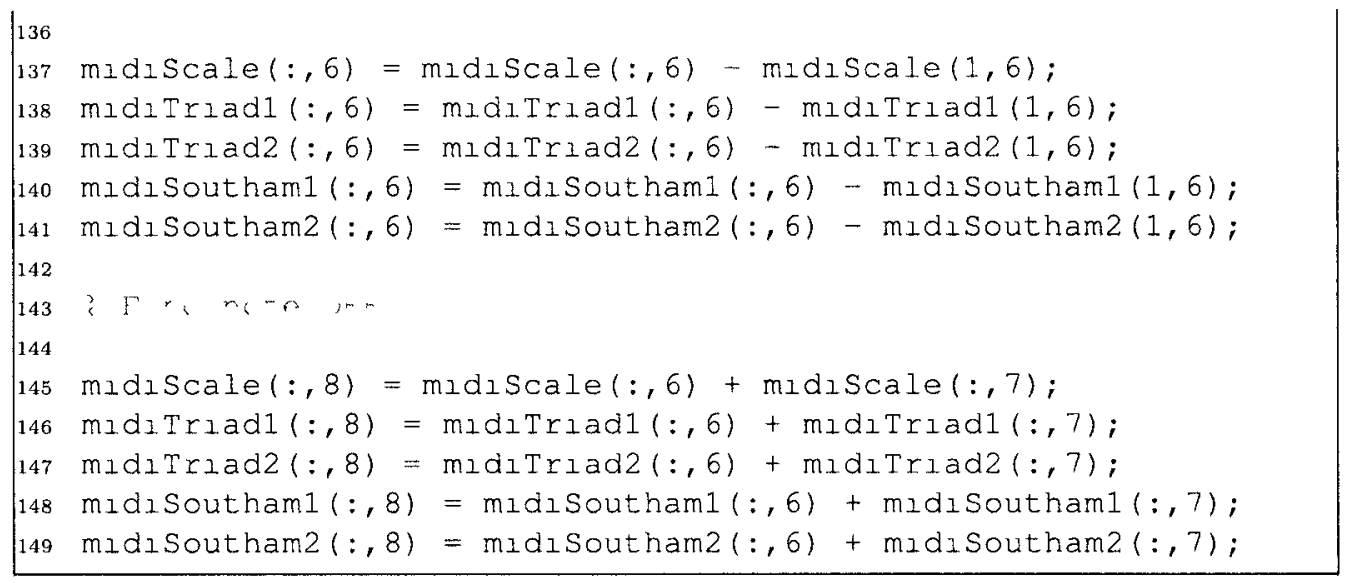




\section{Appendix D}

\section{Code Listing 2: Code for functions used}

\section{in LoadData algorithm}

This appendix contains the code for the functions used in the LoadData algorithm. 


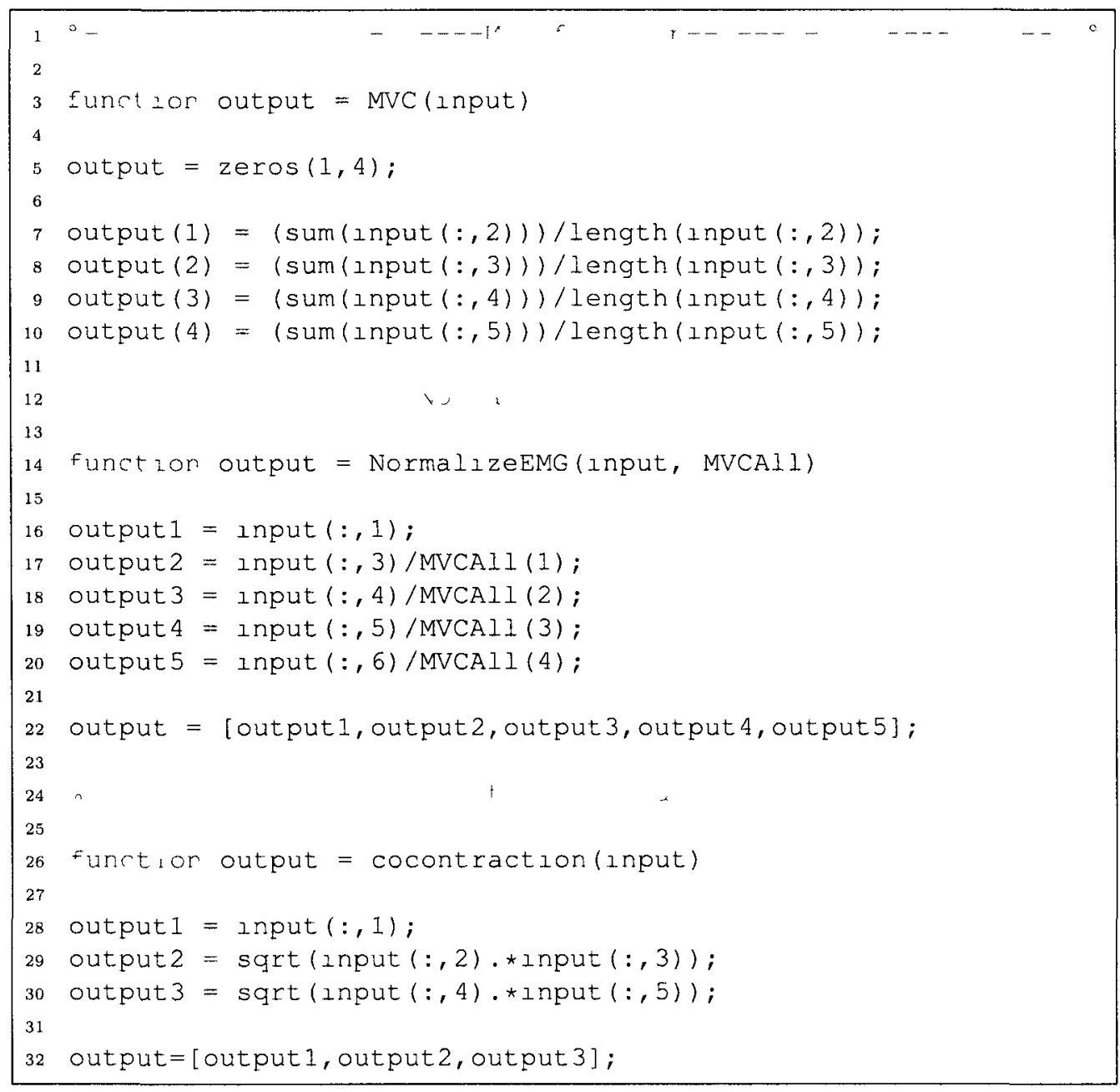




\section{Appendix E}

\section{Code Listing 3: MATLAB commands to}

remove notes played by left hand in Ann

\section{Southam piece from MIDI data}

This $m$ file removes the notes played by the left hand when playing the Ann Southam piece from the MIDI data. It does so by eliminating notes below MIDI note number 60 , as the left hand never plays notes higher than MIDI note number 55 , and the right hand never plays notes below MIDI note number 65. EMG data was collected only from the muscles of the right forearm, thus the notes played by the right hand are useful in the analysis of the Ann Southam piece, but the notes played by the left hand serve no function in the analysis of the EMG data. 


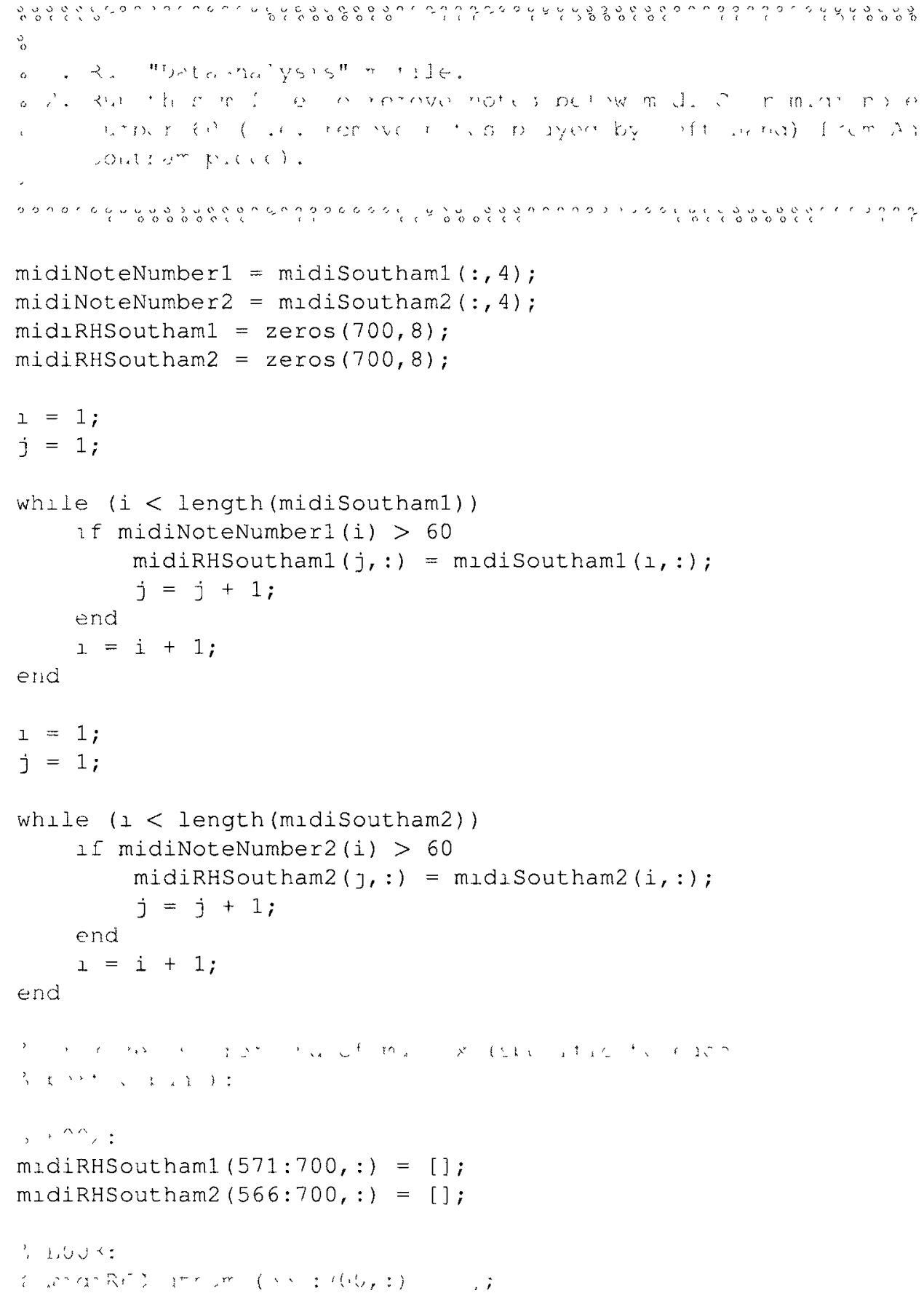




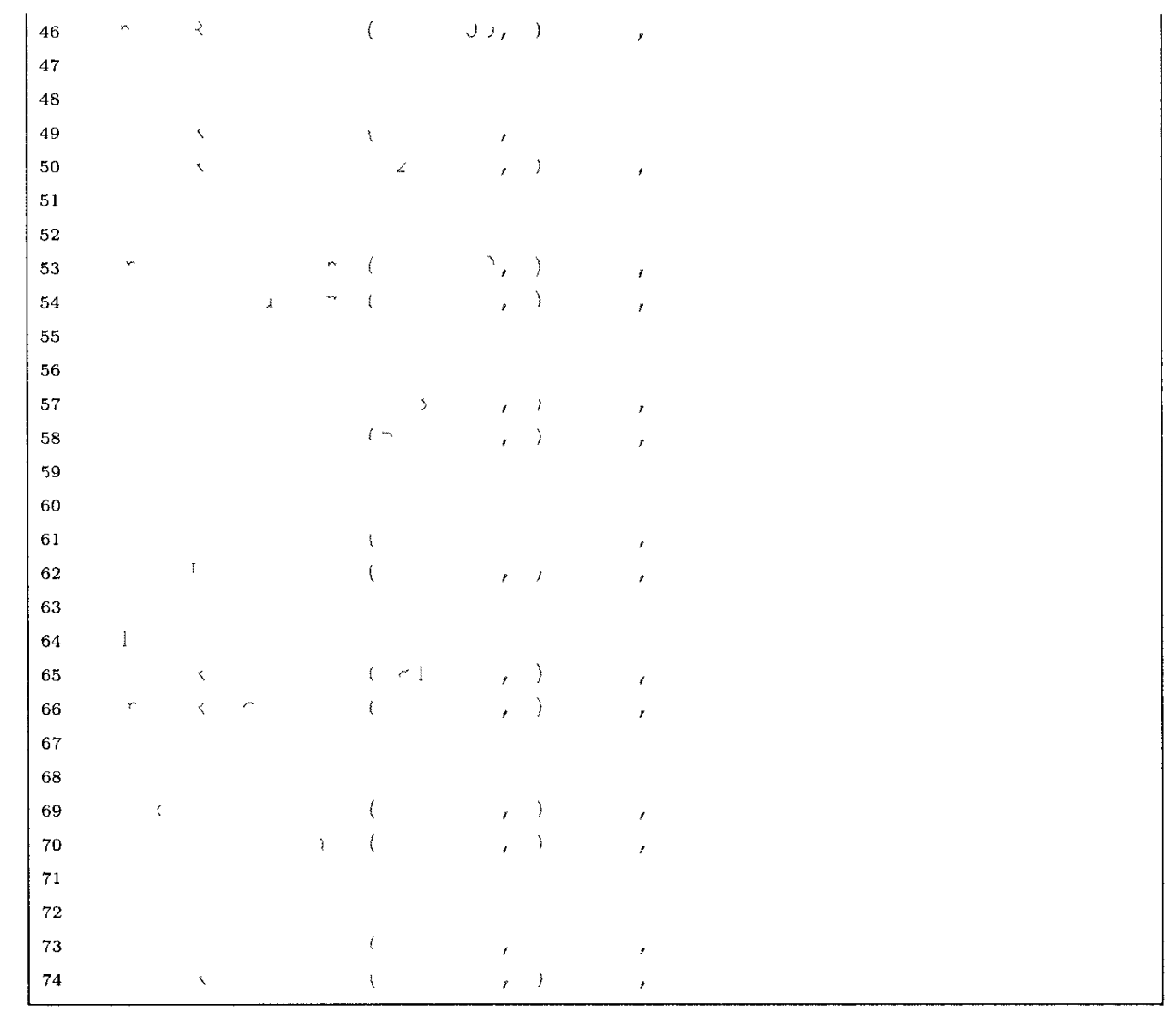




\section{Appendix F}

\section{Code Listing 4: TrimData algorithm}

This $\mathrm{m}$ file removes EMG data from the beginning of EMG files, so that the start of the EMG file corresponds the start of the MIDI file. The code below was used to trim the EMG data of the scale. 


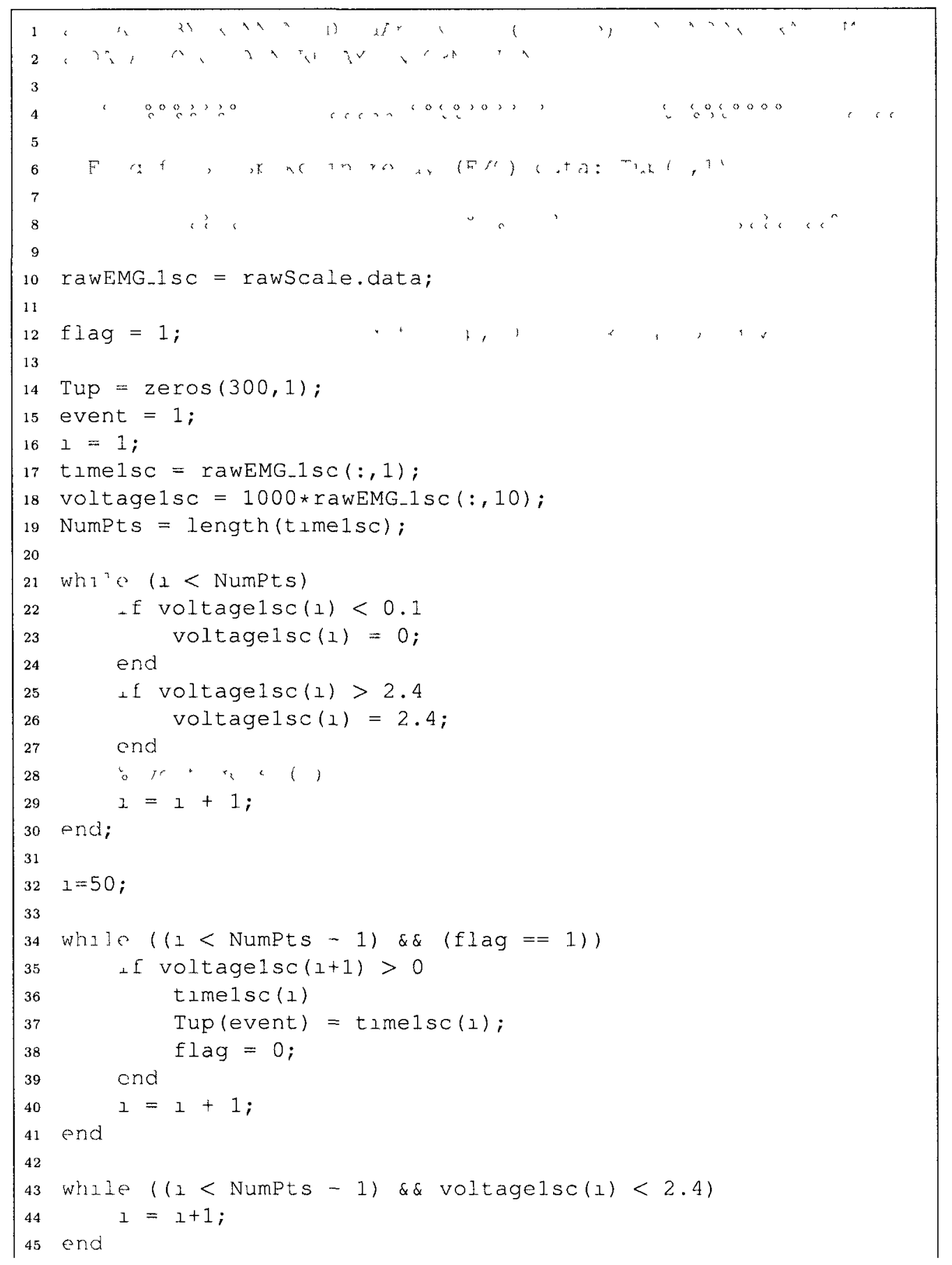




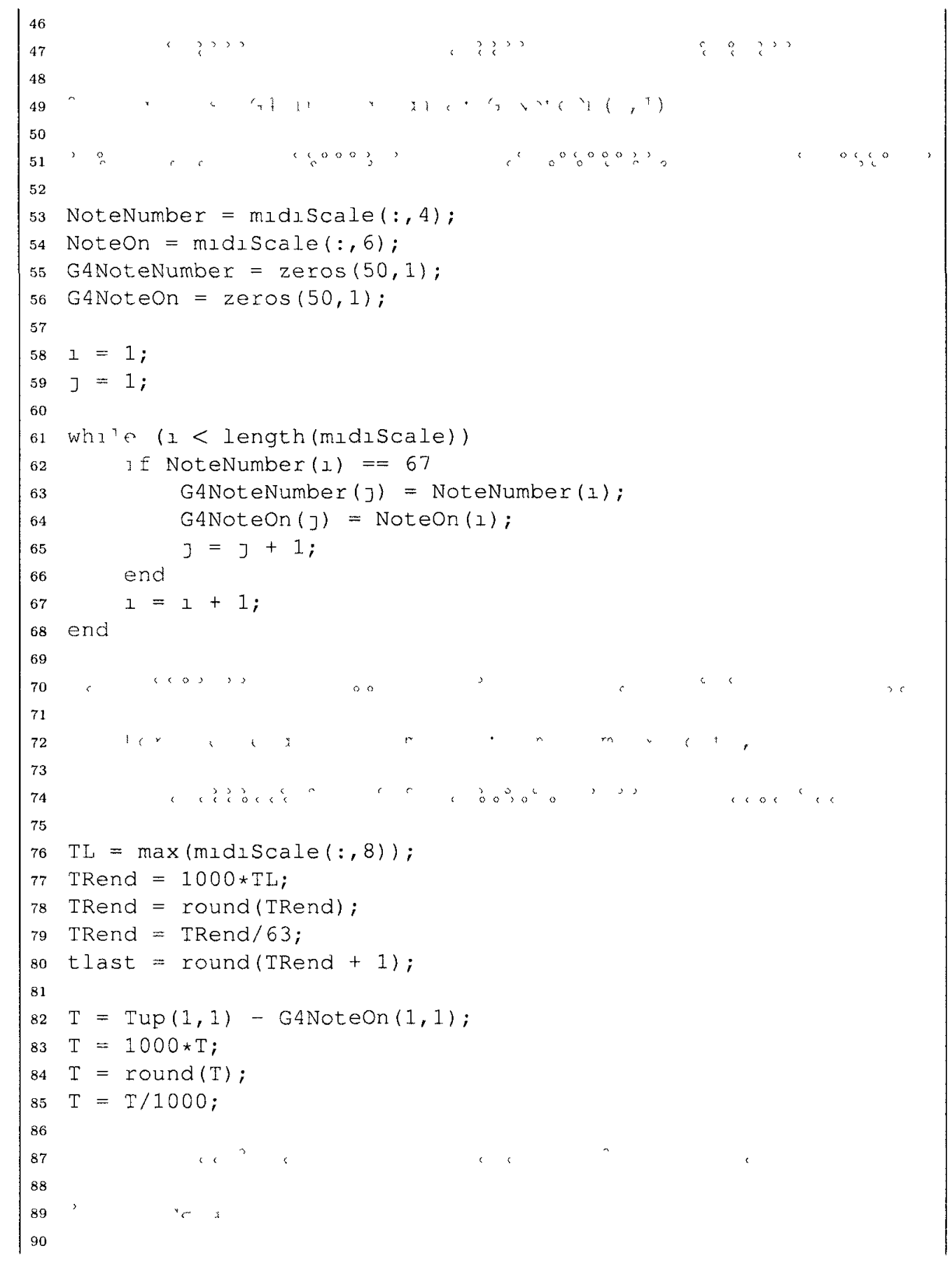




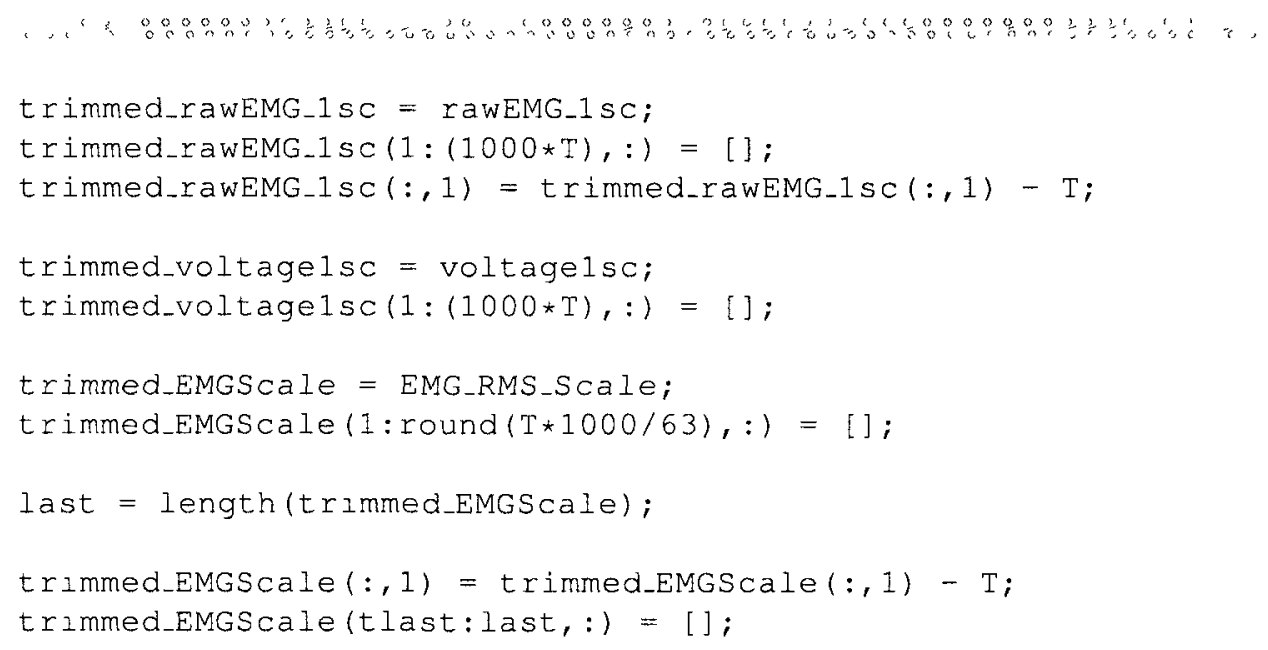




\section{Appendix G}

\section{Code Listing 5: DetectSpike algorithm}

This $\mathrm{m}$ file identifies MIDI note numbers 36 (C2), 48 (C3) and 96 (C7). The locations of these notes in the MIDI matrix identify the times at which the scale changes direction. When $\mathrm{C} 2$ is played, the ascending segment of the scale begins. When $\mathrm{C} 7$ is played, the descending segment of the scale begins. 


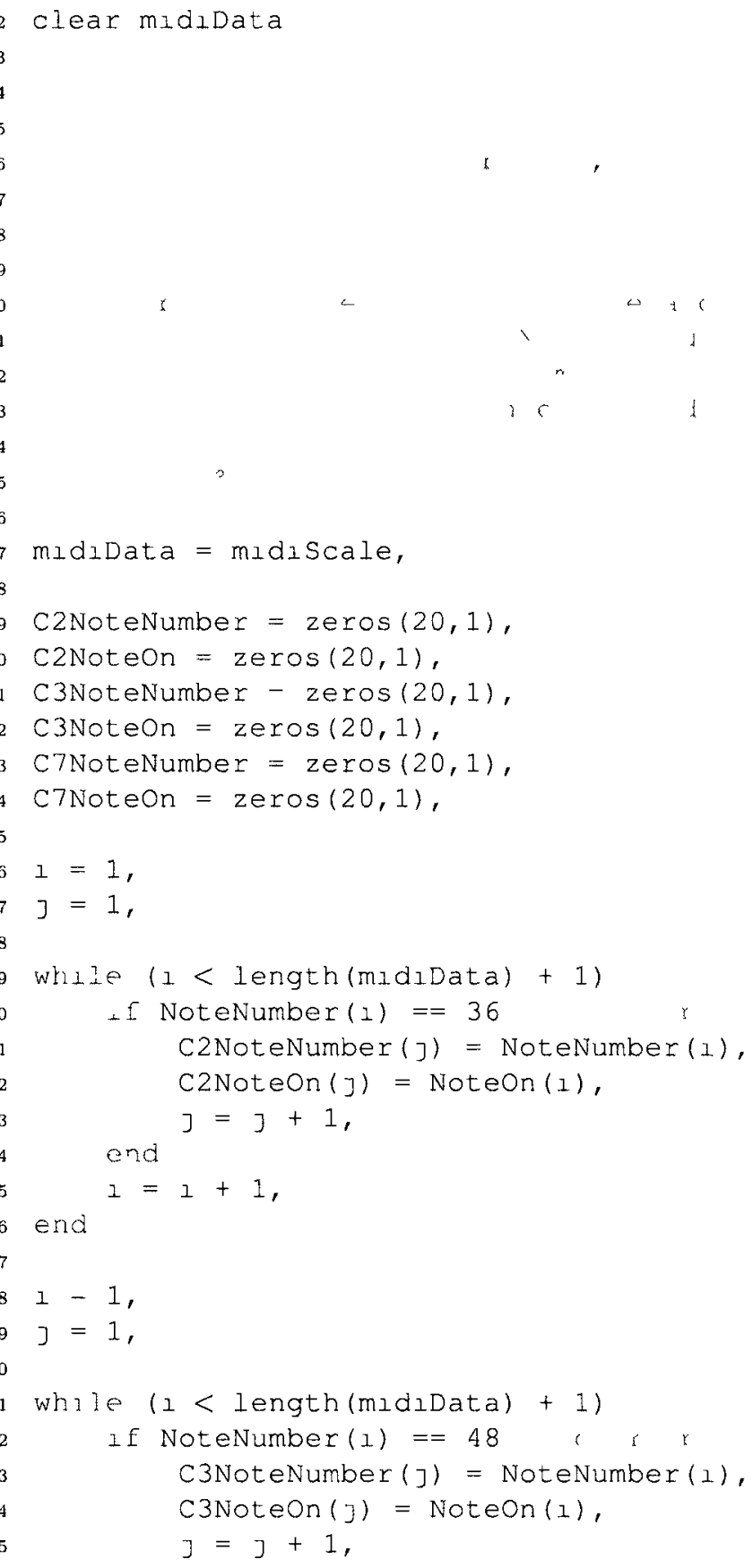




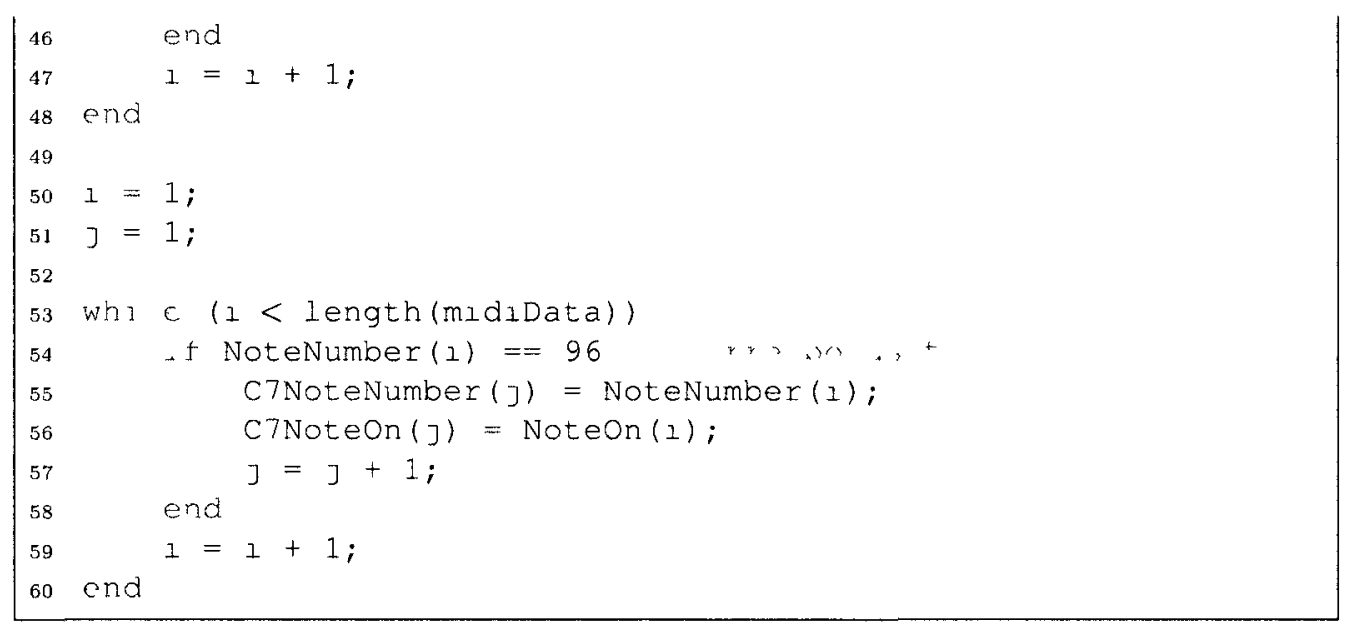


Appendix $\mathbf{H}$

EMG Results of All Participants while

Playing the C Major Scale in Parallel

Motion 

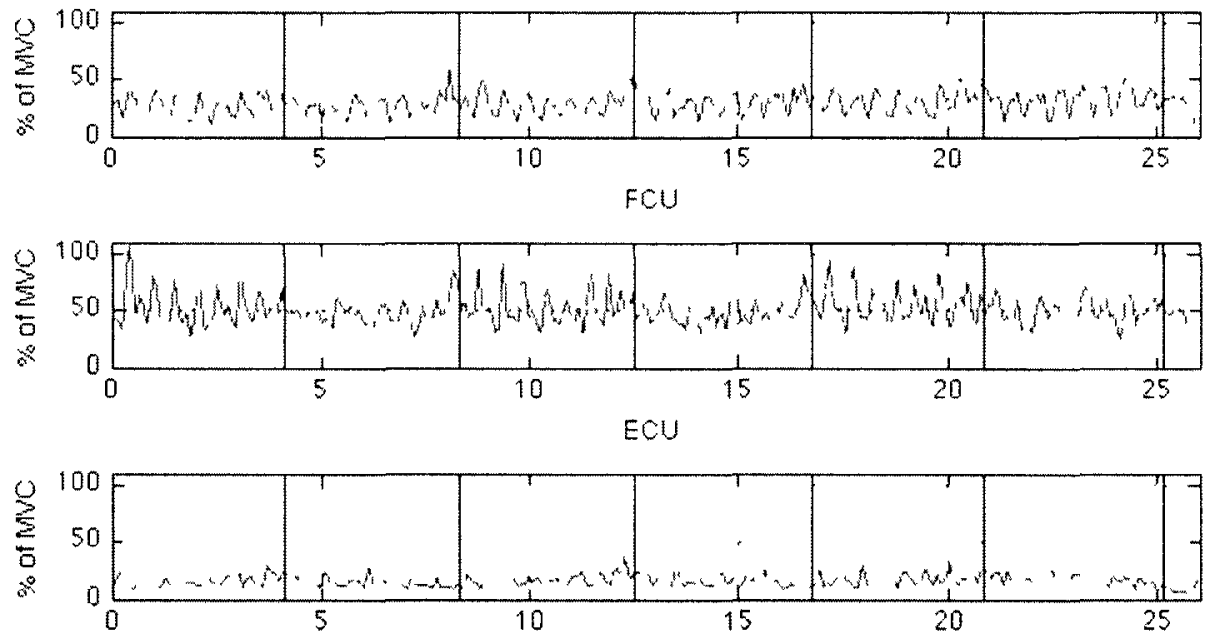

FDS
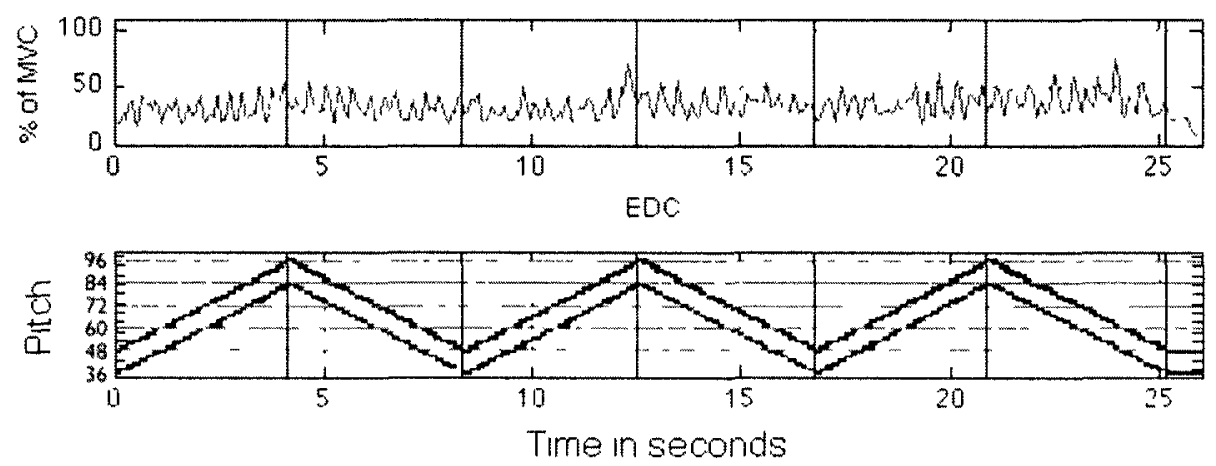

Figure H.1: Participant E002's EMG results: the upper plot shows active muscle stiffness, expressed as a percentage of maximal voluntary contraction while playing the $\mathrm{C}$ major scale in parallel motion, ascending and descending for four muscles of the right forearm: the FCU, the ECU, the FDS and the EDC. The corresponding MIDI data (note on, note off) are shown on the lower plot. 

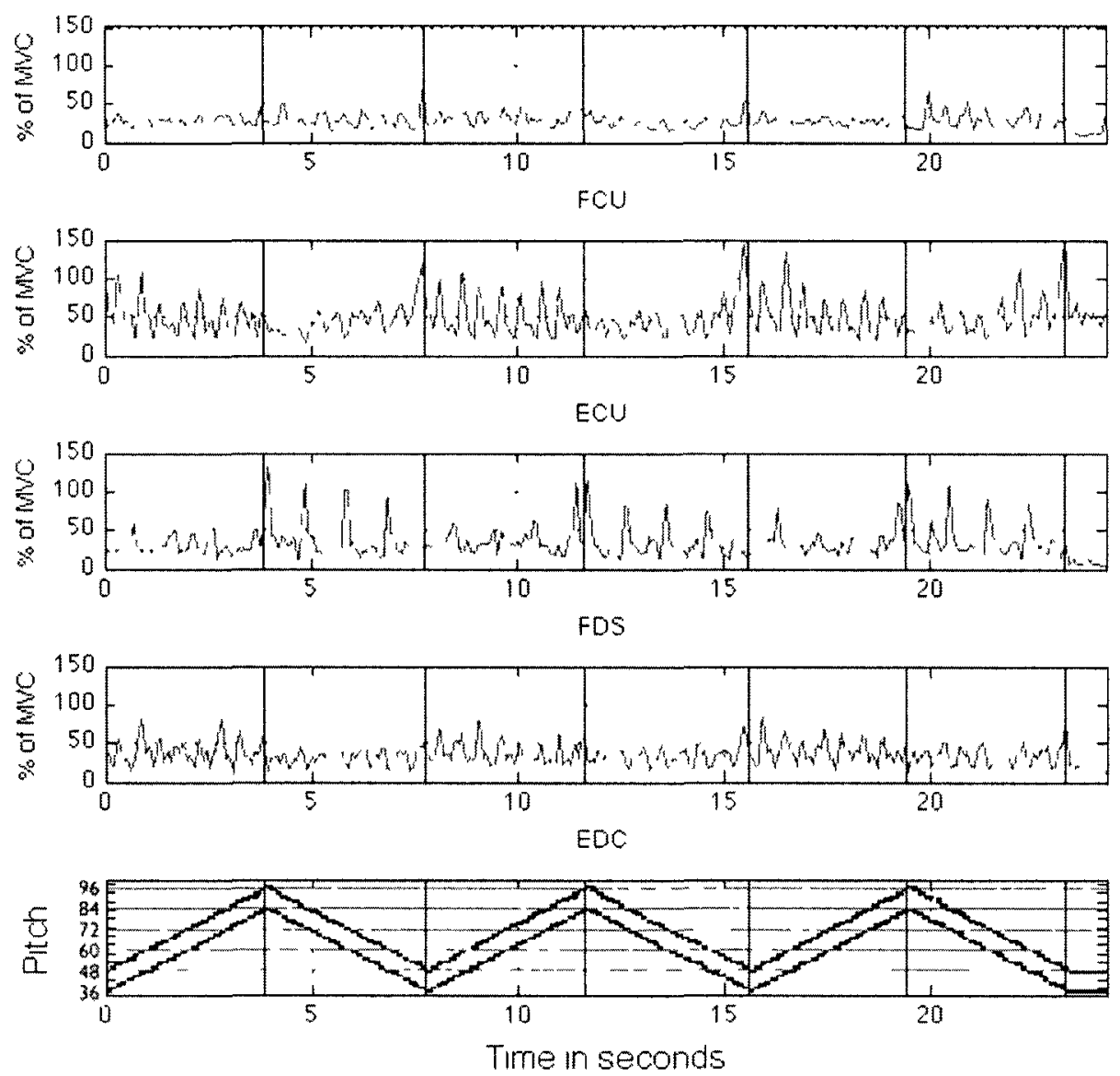

Figure H.2: Participant E003's EMG results: the upper plot shows active muscle stiffness, expressed as a percentage of maximal voluntary contraction while playing the $\mathrm{C}$ major scale in parallel motion, ascending and descending for four muscles of the right forearm: the FCU, the ECU, the FDS and the EDC The corresponding MIDI data (note on, note off) are shown on the lower plot. 

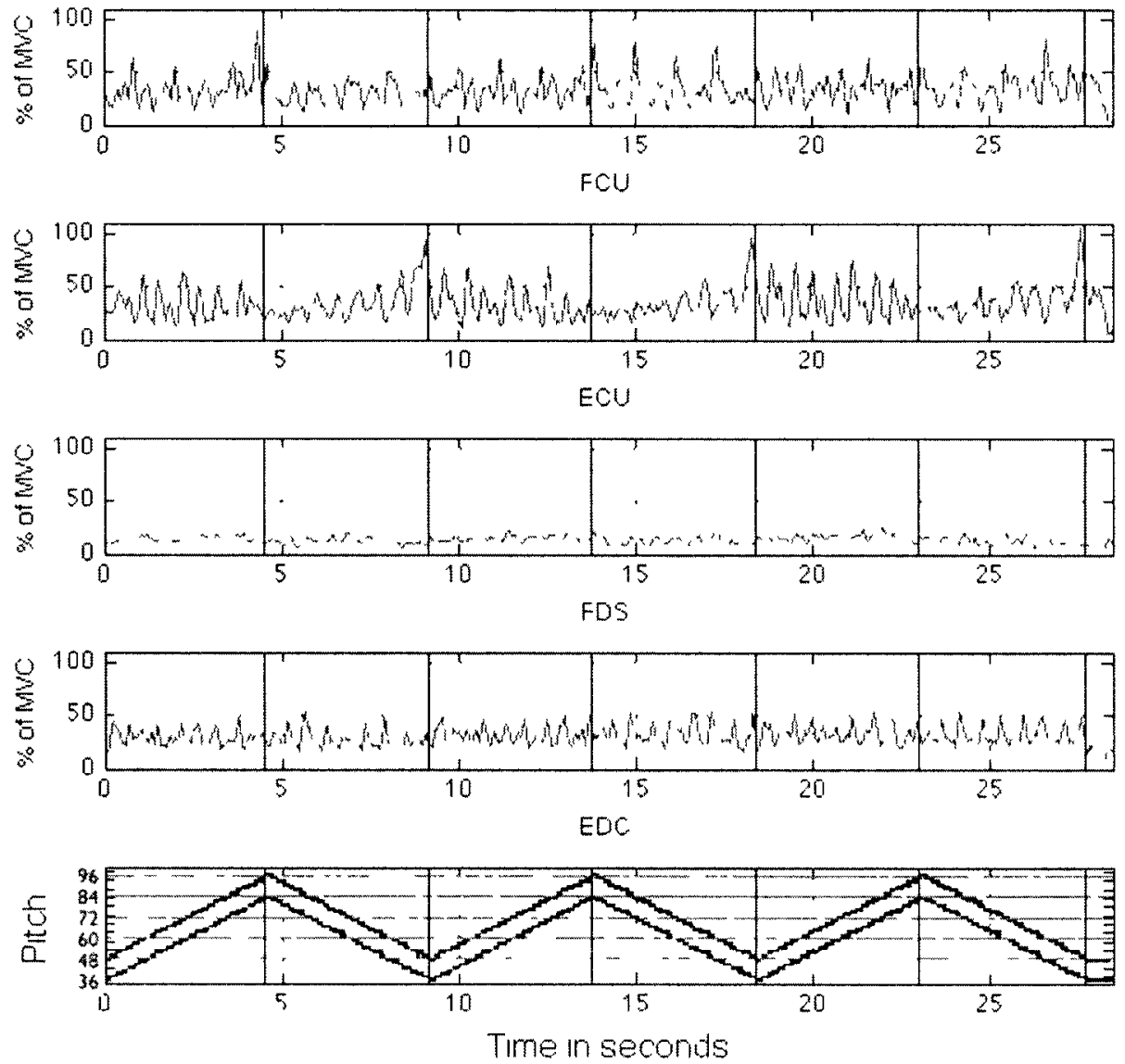

Figure H.3: Participant E004's EMG results: the upper plot shows active muscle stiffness, expressed as a percentage of maximal voluntary contraction while playing the $\mathrm{C}$ major scale in parallel motion, ascending and descending for four muscles of the right forearm: the FCU, the ECU, the FDS and the EDC. The corresponding MIDI data (note on, note off) are shown on the lower plot. 

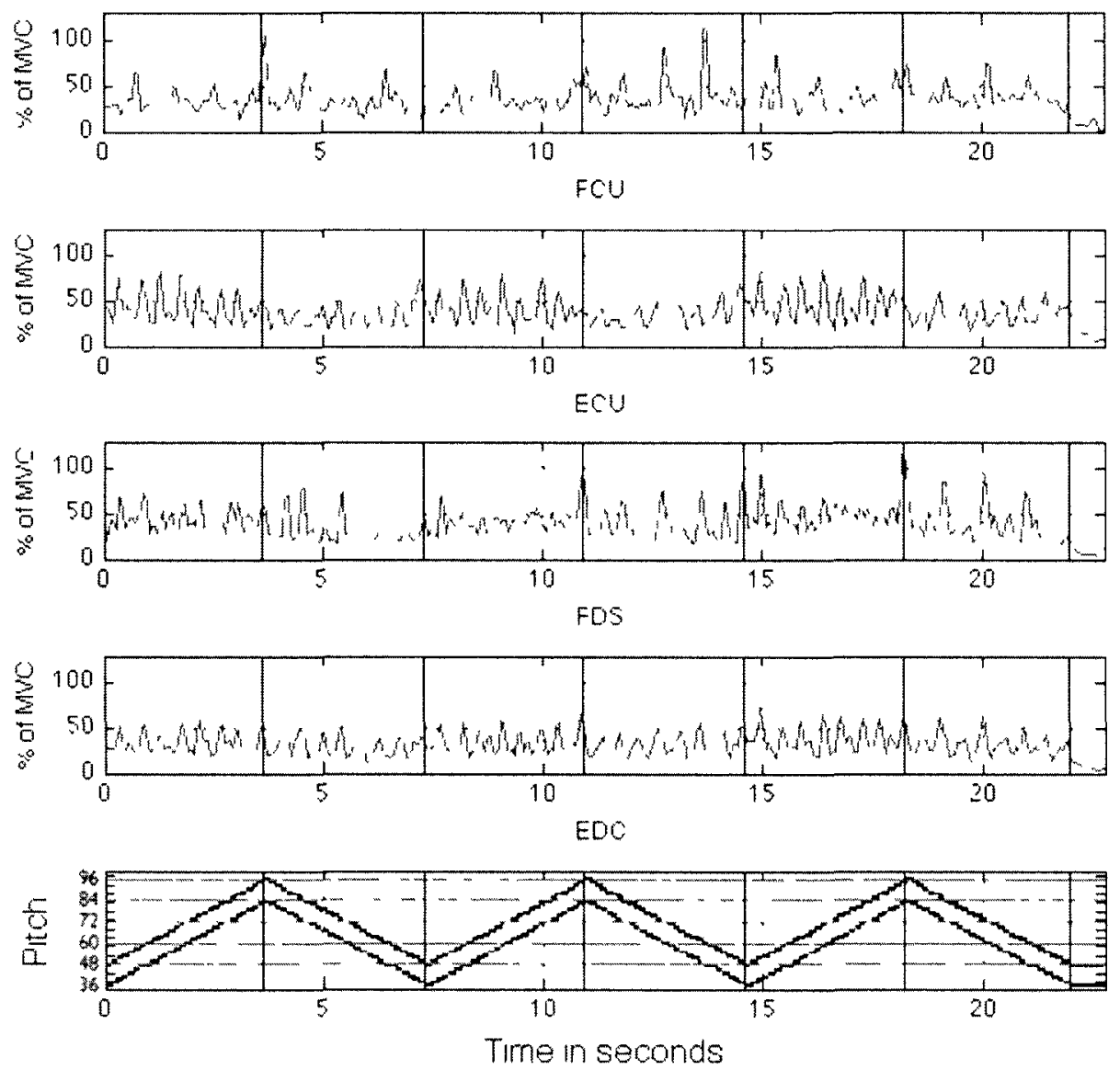

Figure H.4: Participant E005's EMG results the upper plot shows active muscle stiffness, expressed as a percentage of maximal voluntary contraction while playing the $\mathrm{C}$ major scale in parallel motion, ascending and descending for four muscles of the right forearm. the FCU, the ECU, the FDS and the EDC. The corresponding MIDI data (note on, note off) are shown on the lower plot. 

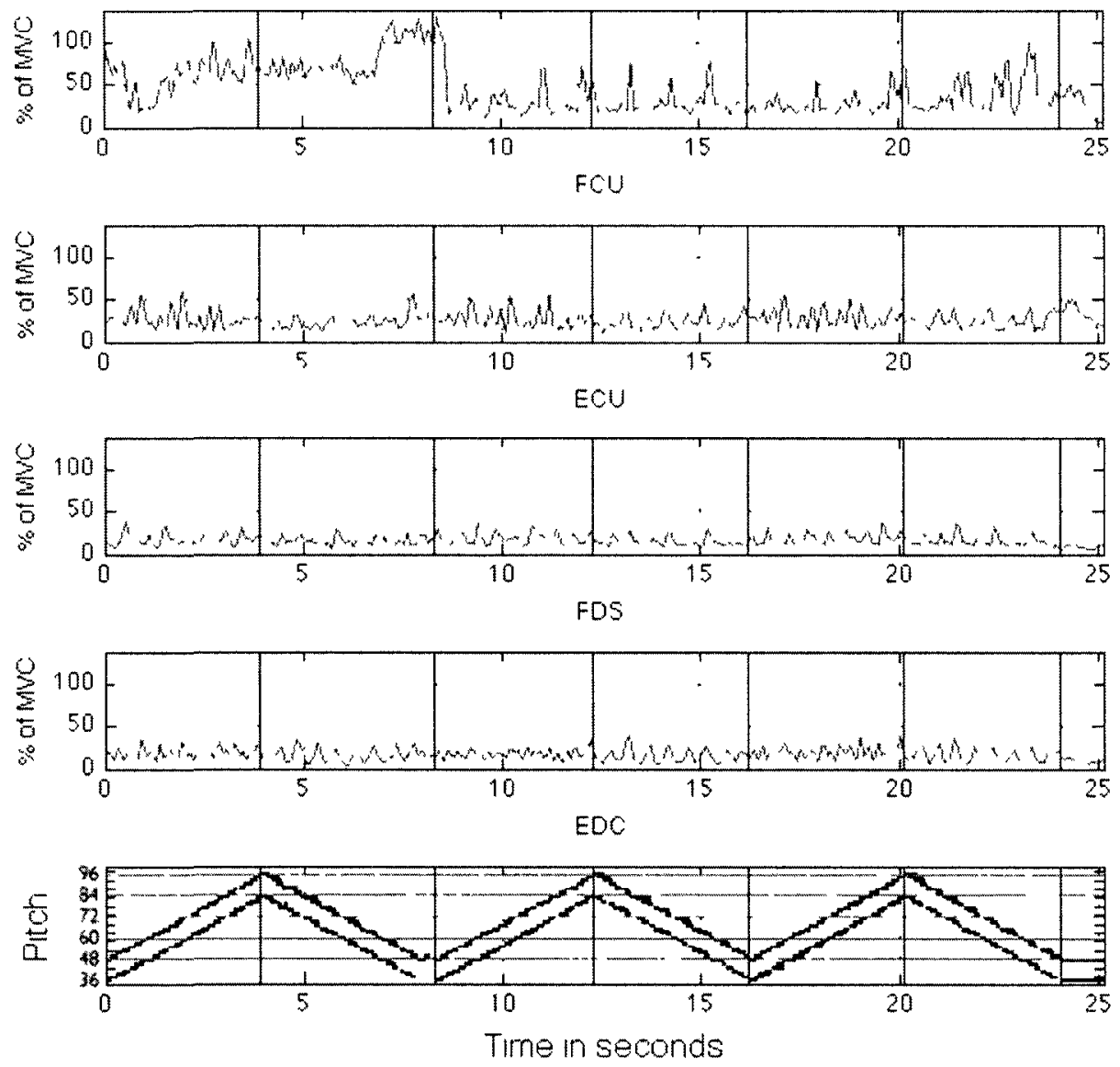

Figure H.5: Participant E006's EMG results: the upper plot shows active muscle stiffness, expressed as a percentage of maximal voluntary contraction while playing the $\mathrm{C}$ major scale in parallel motion, ascending and descending for four muscles of the right forearm: the FCU, the ECU, the FDS and the EDC. Initially (approximately the first $8.5 \mathrm{~s}$ ), the wire connecting the FCU sensor to the main amplifier interfered with the FCU electrodes, yielding measurements that do not accurately reflect the level of active muscle stiffness. The contaminated data are shown in this figure, but these data are not included in any of the analyses. The corresponding MIDI data (note on, note off) are shown on the lower plot. 

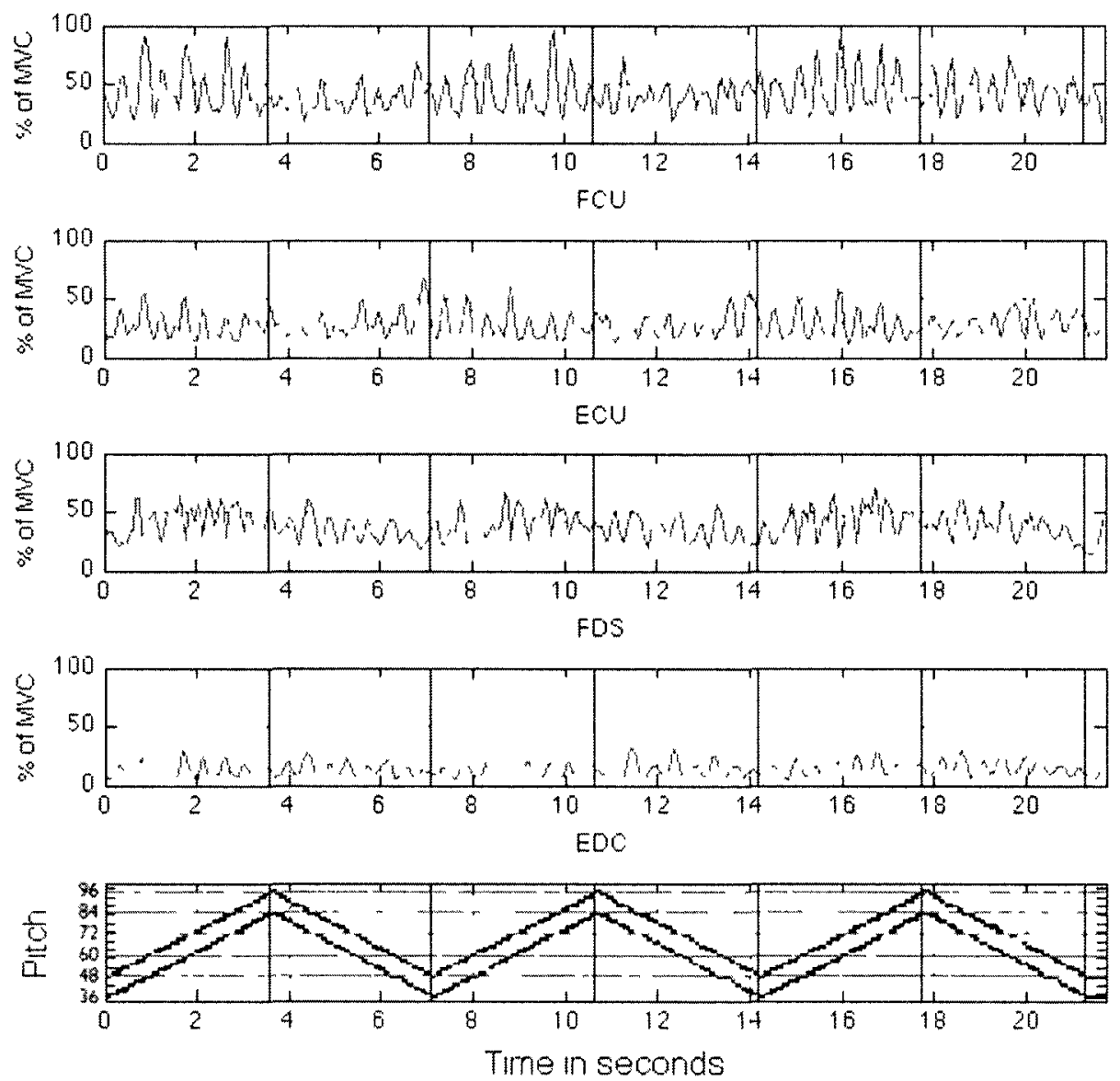

Figure H.6: Participant E007's EMG results: the upper plot shows active muscle stiffness, expressed as a percentage of maximal voluntary contraction while playing the $\mathrm{C}$ major scale in parallel motion, ascending and descending for four muscles of the right forearm: the FCU, the ECU, the FDS and the EDC. The corresponding MIDI data (note on, note off) are shown on the lower plot. 

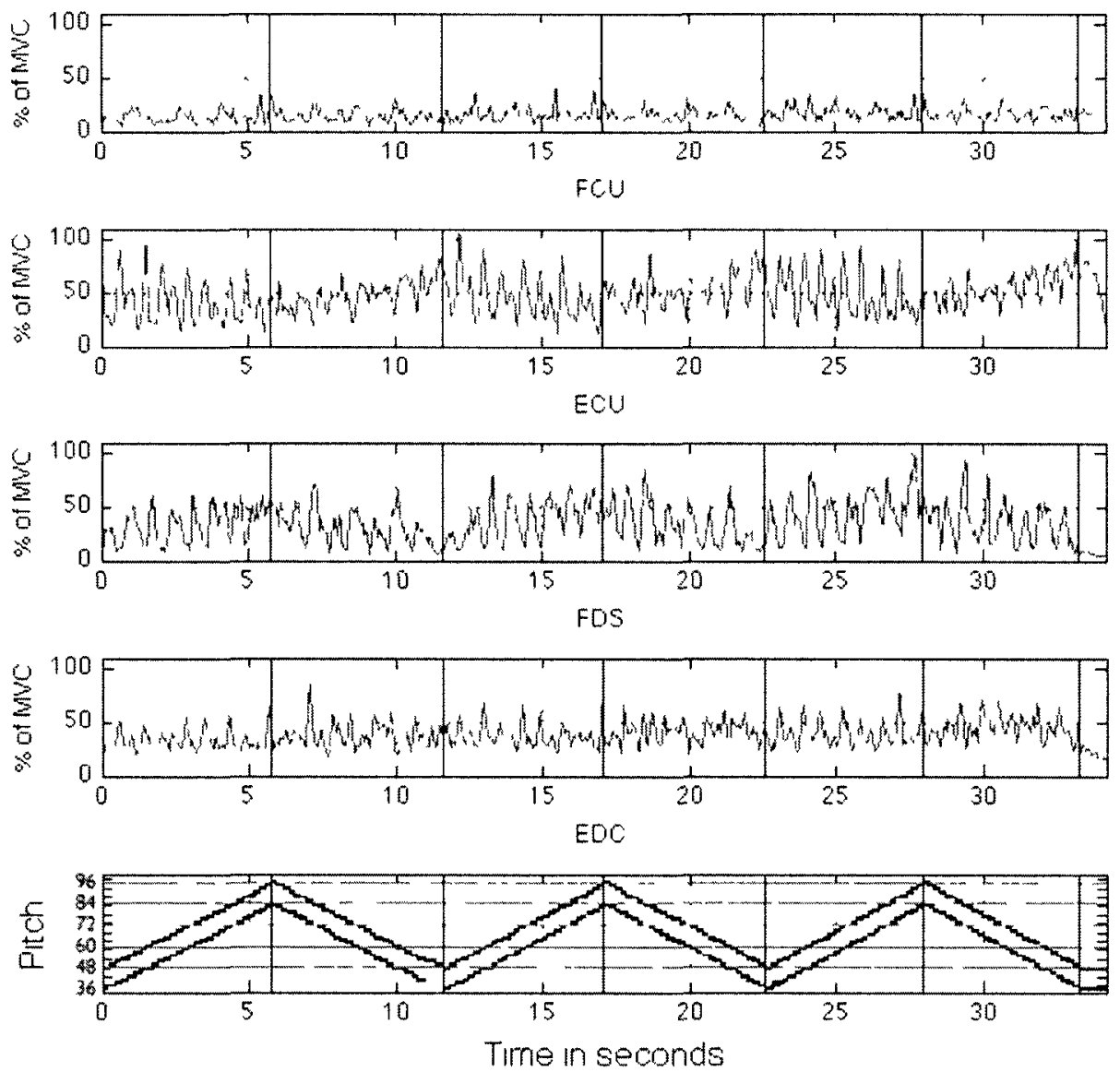

Figure H.7: Participant E009's EMG results: the upper plot shows active muscle stiffness, expressed as a percentage of maximal voluntary contraction while playing the $\mathrm{C}$ major scale in parallel motion, ascending and descending for four muscles of the right forearm: the FCU, the ECU, the FDS and the EDC. The corresponding MIDI data (note on, note off) are shown on the lower plot. 

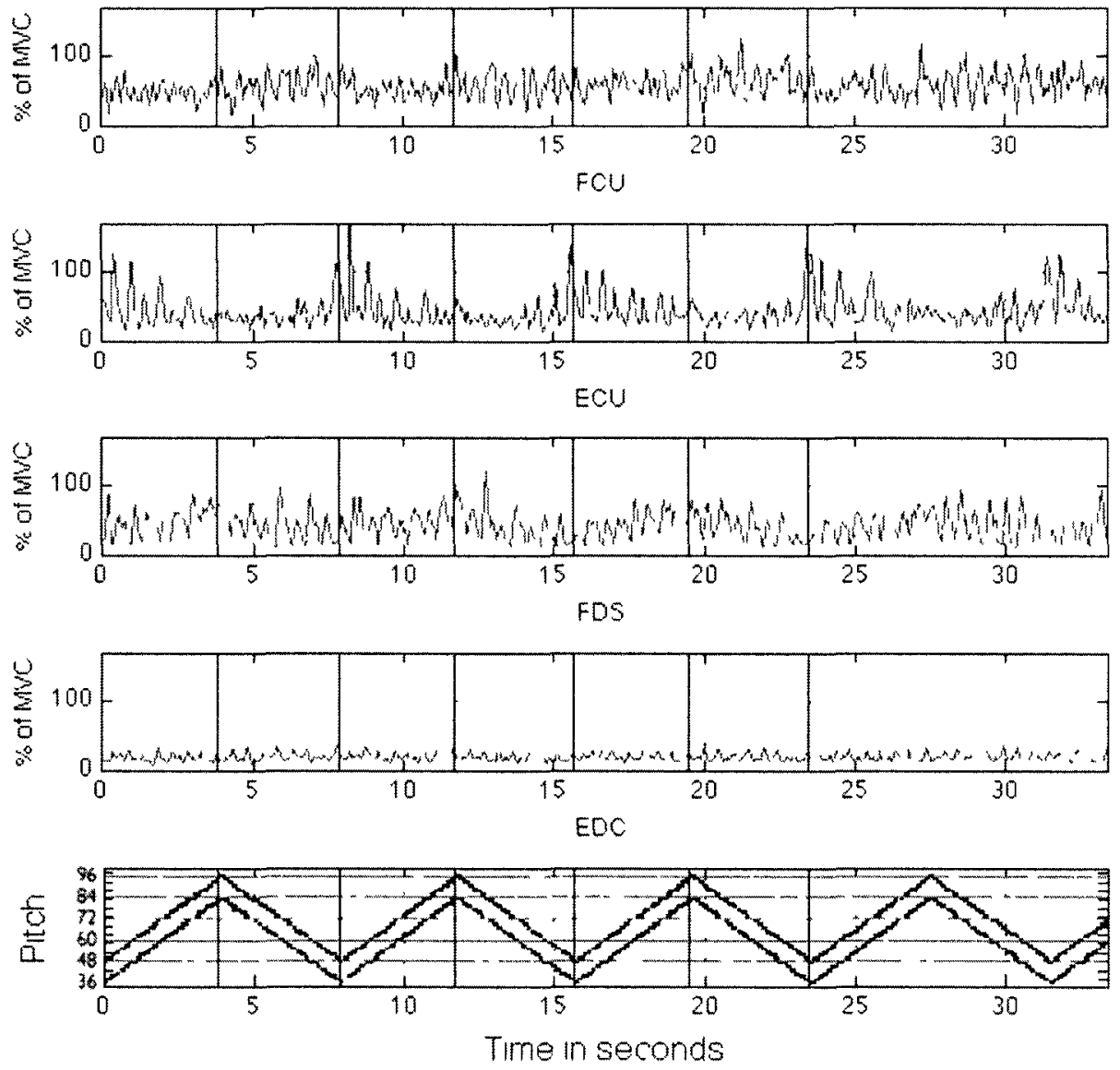

Figure H.8: Participant E010's EMG results, the upper plot shows active muscle stiffness, expressed as a percentage of maximal voluntary contraction while playing the $\mathrm{C}$ major scale in parallel motion, ascending and descending for four muscles of the right forearm: the FCU, the ECU, the FDS and the EDC. The corresponding MIDI data (note on, note off) are shown on the lower plot. Participant E010 played the scale, both ascending and descending segments, an additional time, and began to play the scale for a fifth time before being told to stop. The data collected during the playing of the additional octaves are shown in the figure; however, only the first three octaves are used in the analyses of the scale. 

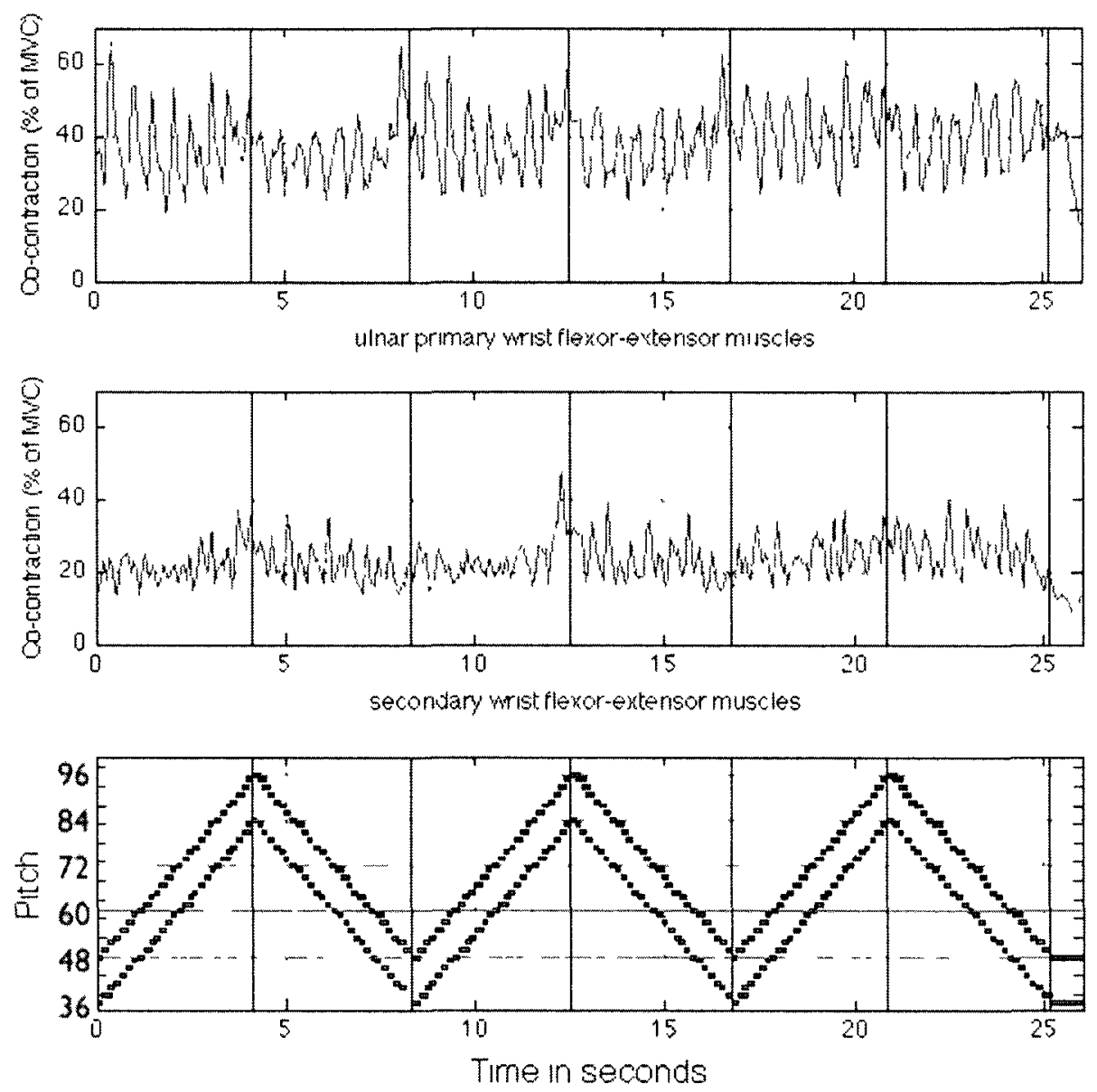

Figure H.9: Participant E002's results: the upper plot shows levels of muscular cocontraction, expressed as a percentage of maximal voluntary contraction, while playing the $\mathrm{C}$ major scale in parallel motion, ascending and descending. The corresponding MIDI data (note on, note off) are shown on the lower plot. 

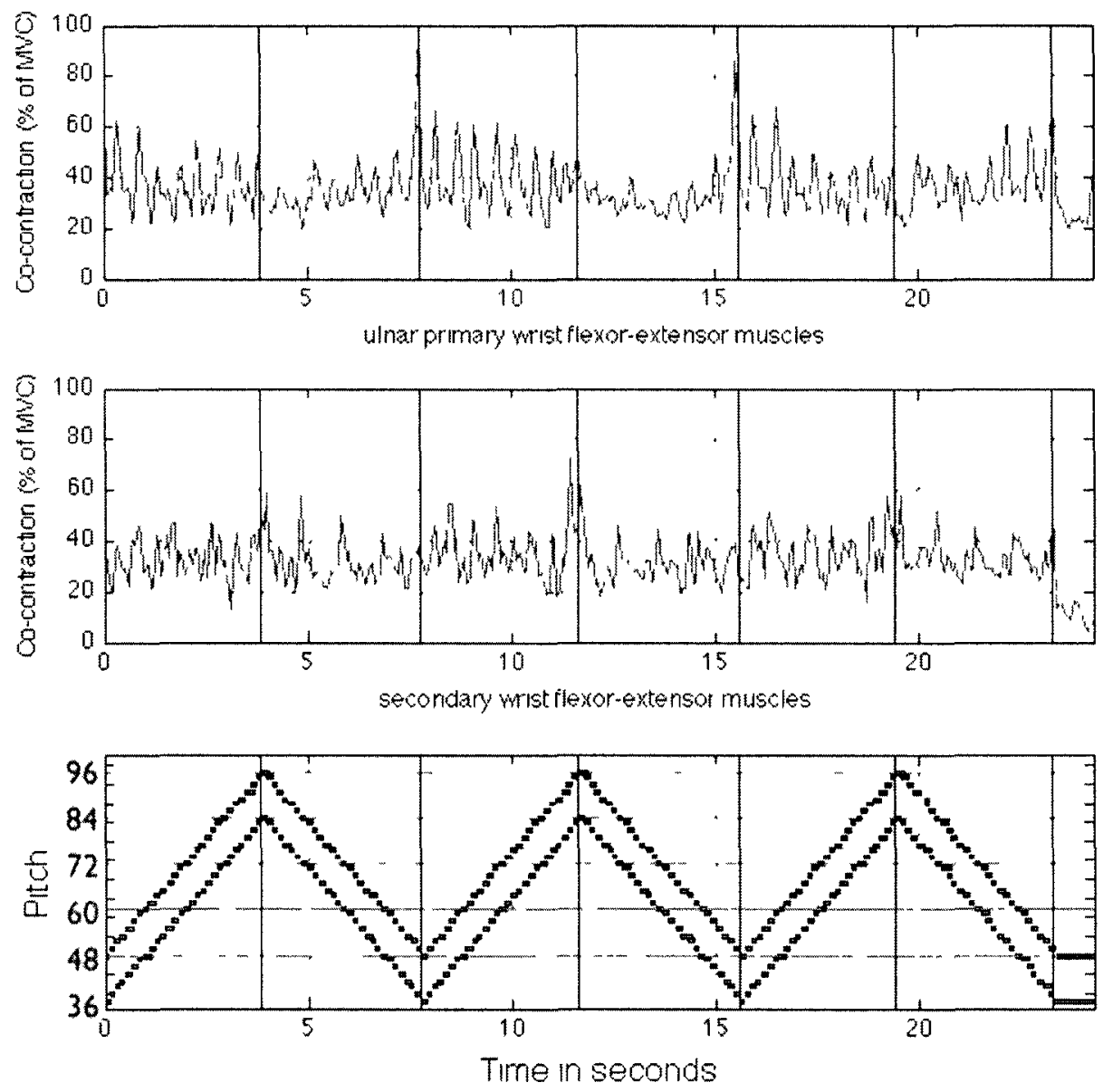

Figure H.10: Participant E003's results: the upper plot shows levels of muscular cocontraction, expressed as a percentage of maximal voluntary contraction, while playing the $\mathrm{C}$ major scale in parallel motion, ascending and descending. The corresponding MIDI data (note on, note off) are shown on the lower plot. 

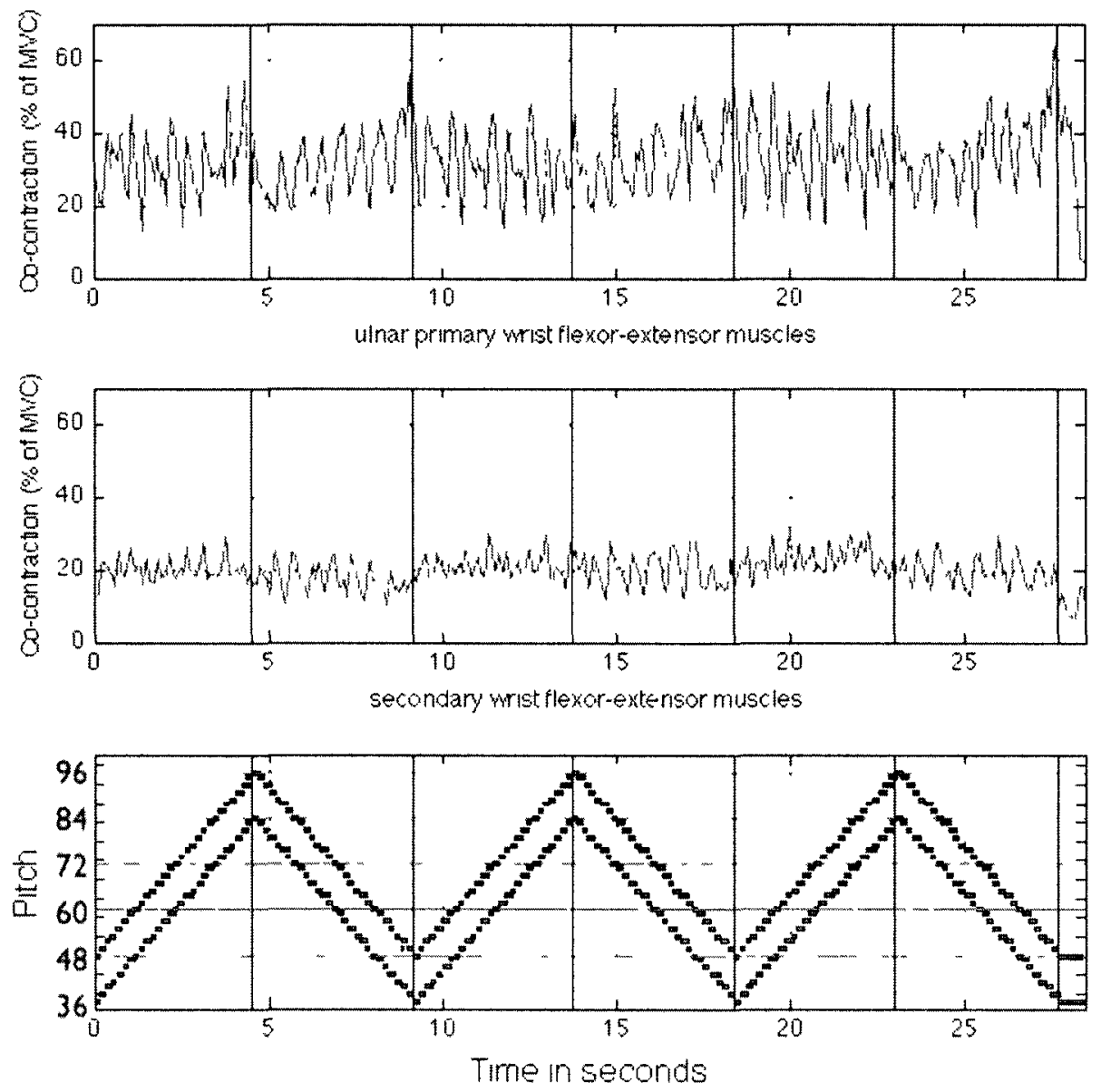

Figure H.11: Participant E004's results: the upper plot shows levels of muscular cocontraction, expressed as a percentage of maximal voluntary contraction, while playing the $\mathrm{C}$ major scale in parallel motion, ascending and descending. The corresponding MIDI data (note on, note off) are shown on the lower plot. 

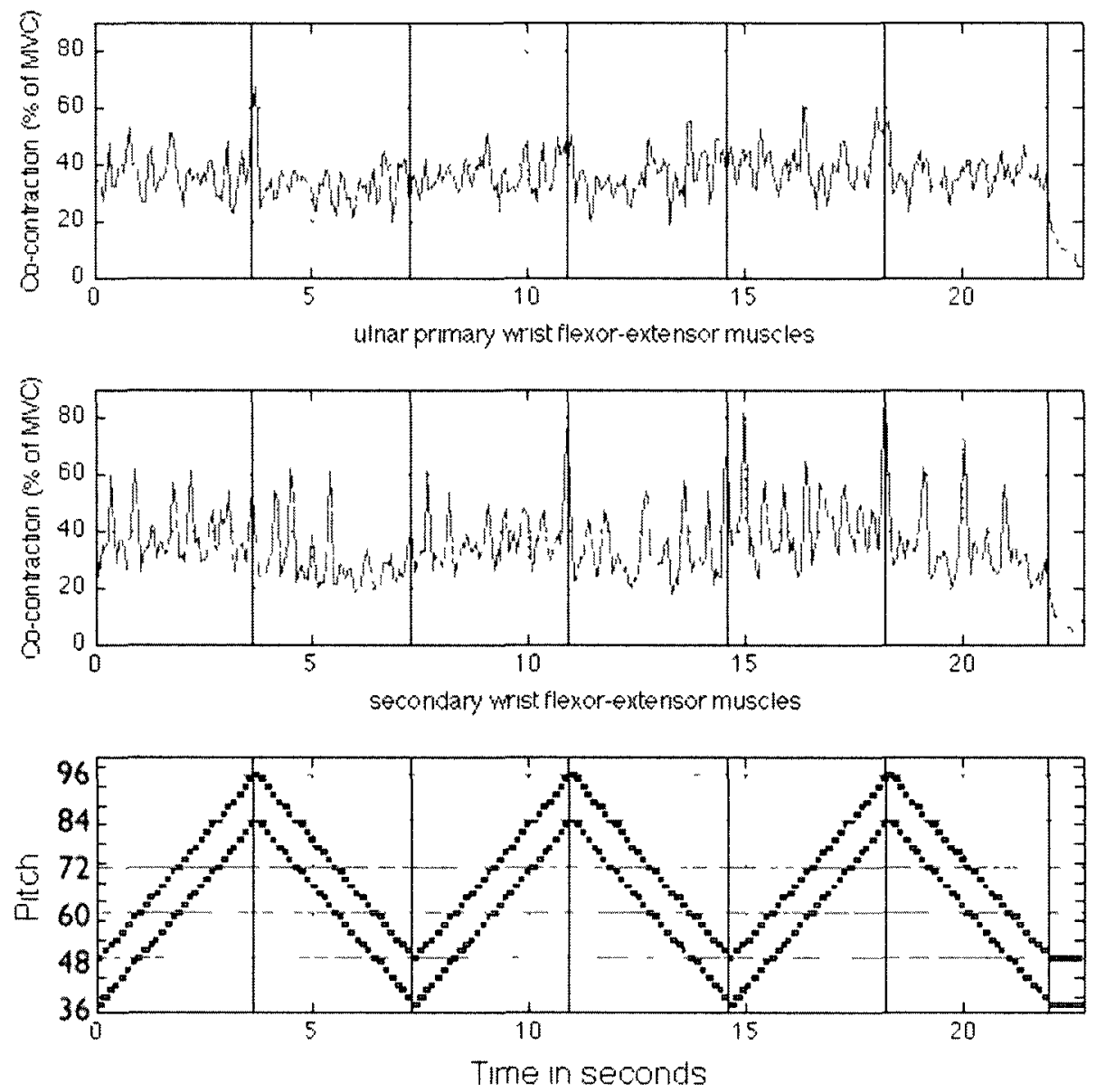

Figure H.12: Participant E005's results: the upper plot shows levels of muscular cocontraction, expressed as a percentage of maximal voluntary contraction, while playing the $\mathrm{C}$ major scale in parallel motion, ascending and descending. The corresponding MIDI data (note on, note off) are shown on the lower plot. 

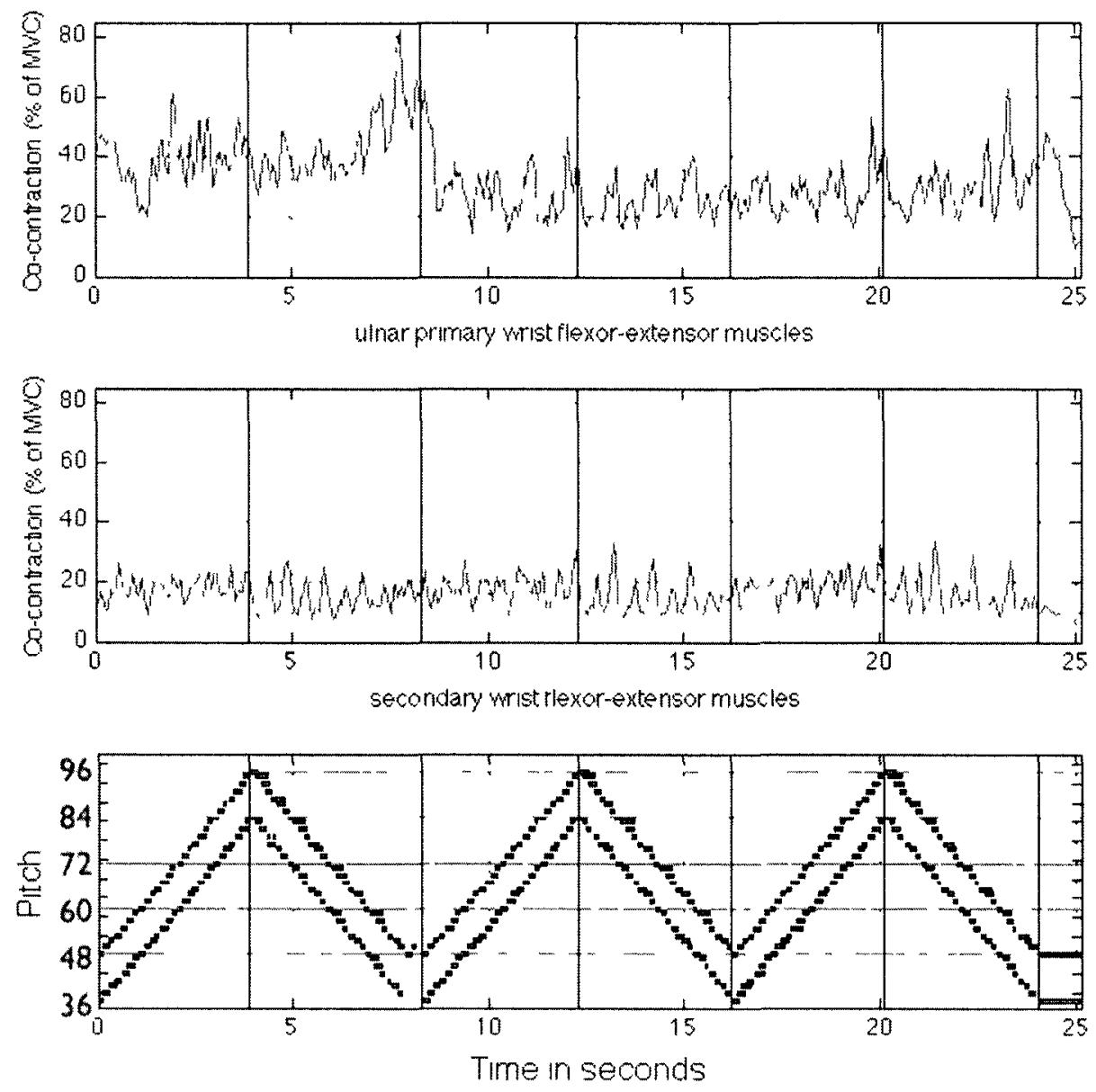

Figure H.13: Partıcipant E006's results: the upper plot shows levels of muscular co-contraction, expressed as a percentage of maximal voluntary contraction, while playing the $\mathrm{C}$ major scale in parallel motion, ascending and descending. Initially (approximately the first $8.5 \mathrm{~s}$ ), the wire connecting the FCU sensor to the main amplifier interfered with the FCU electrodes, yielding measurements that do not accurately reflect the level of active muscle stiffness, and thus preventing an accurate measurement of co-contraction. The contaminated data are shown in this figure, but these data are not included in any of the analyses. The corresponding MIDI data (note on, note off) are shown on the lower plot. 

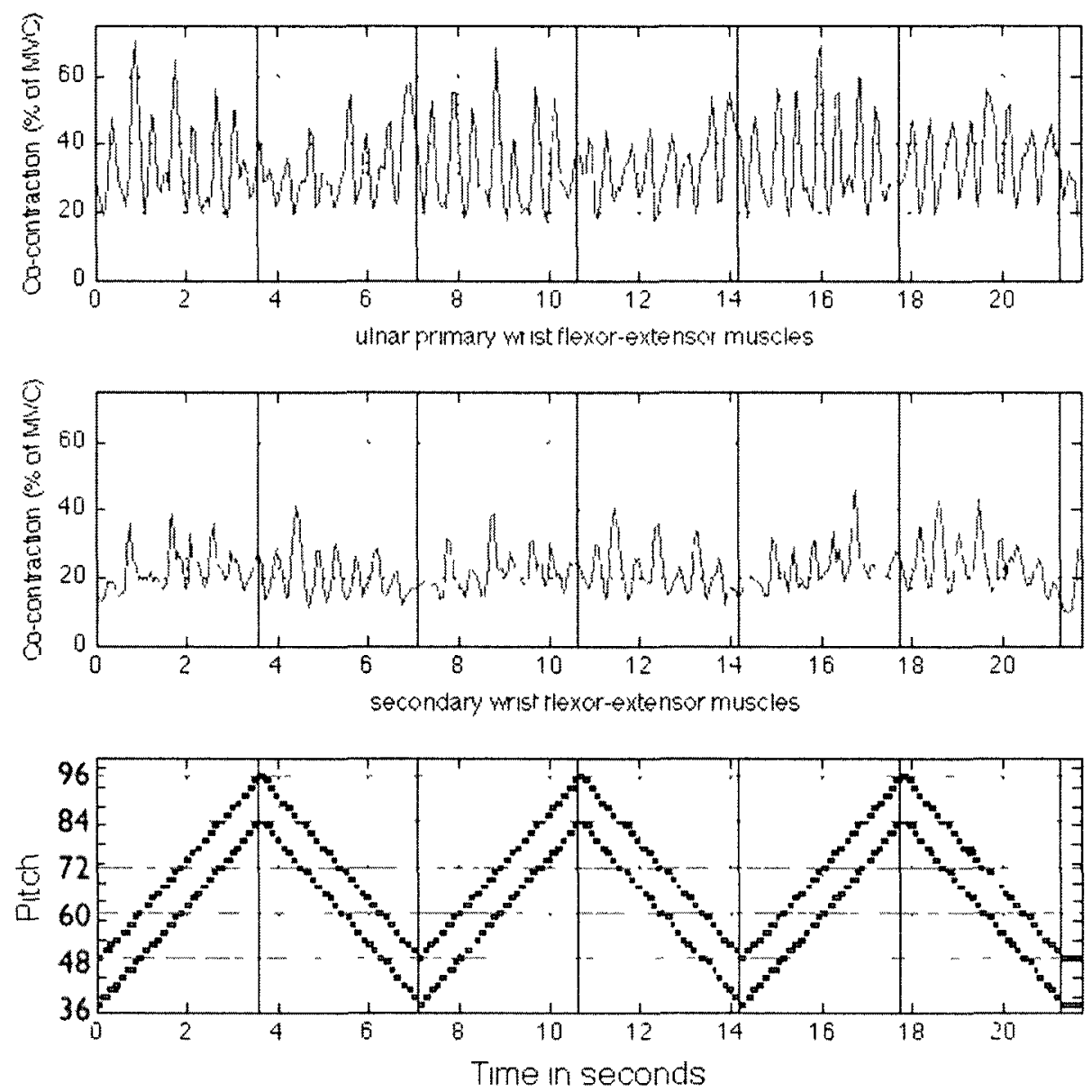

Figure H.14: Participant E007's results: the upper plot shows levels of muscular cocontraction, expressed as a percentage of maximal voluntary contraction, while playing the $\mathrm{C}$ major scale in parallel motion, ascending and descending. The corresponding MIDI data (note on, note off) are shown on the lower plot. 

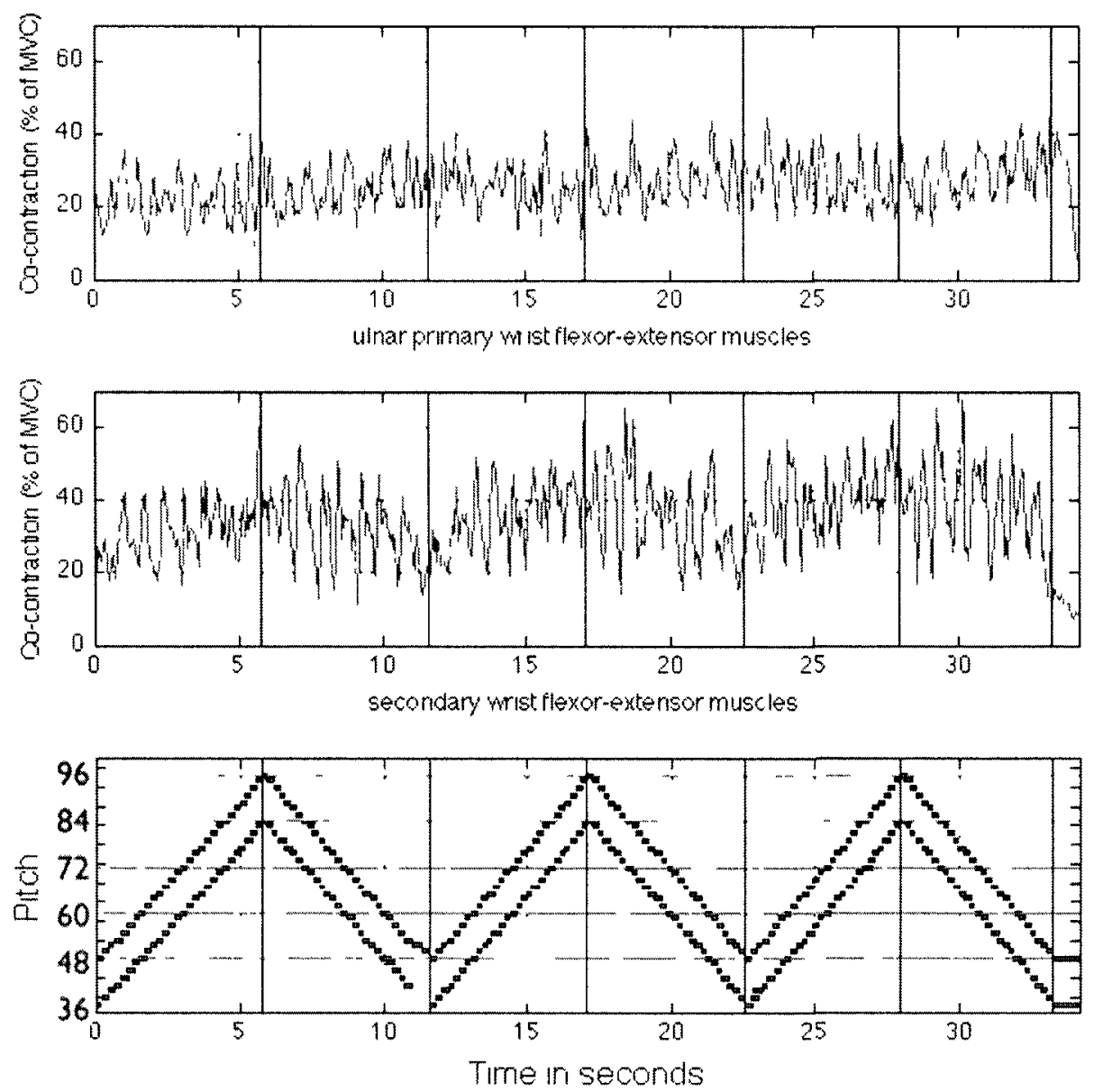

Figure H.15: Participant E009's results: the upper plot shows levels of muscular cocontraction, expressed as a percentage of maximal voluntary contraction, while playing the $\mathrm{C}$ major scale in parallel motion, ascending and descending. The corresponding MIDI data (note on, note off) are shown on the lower plot. 

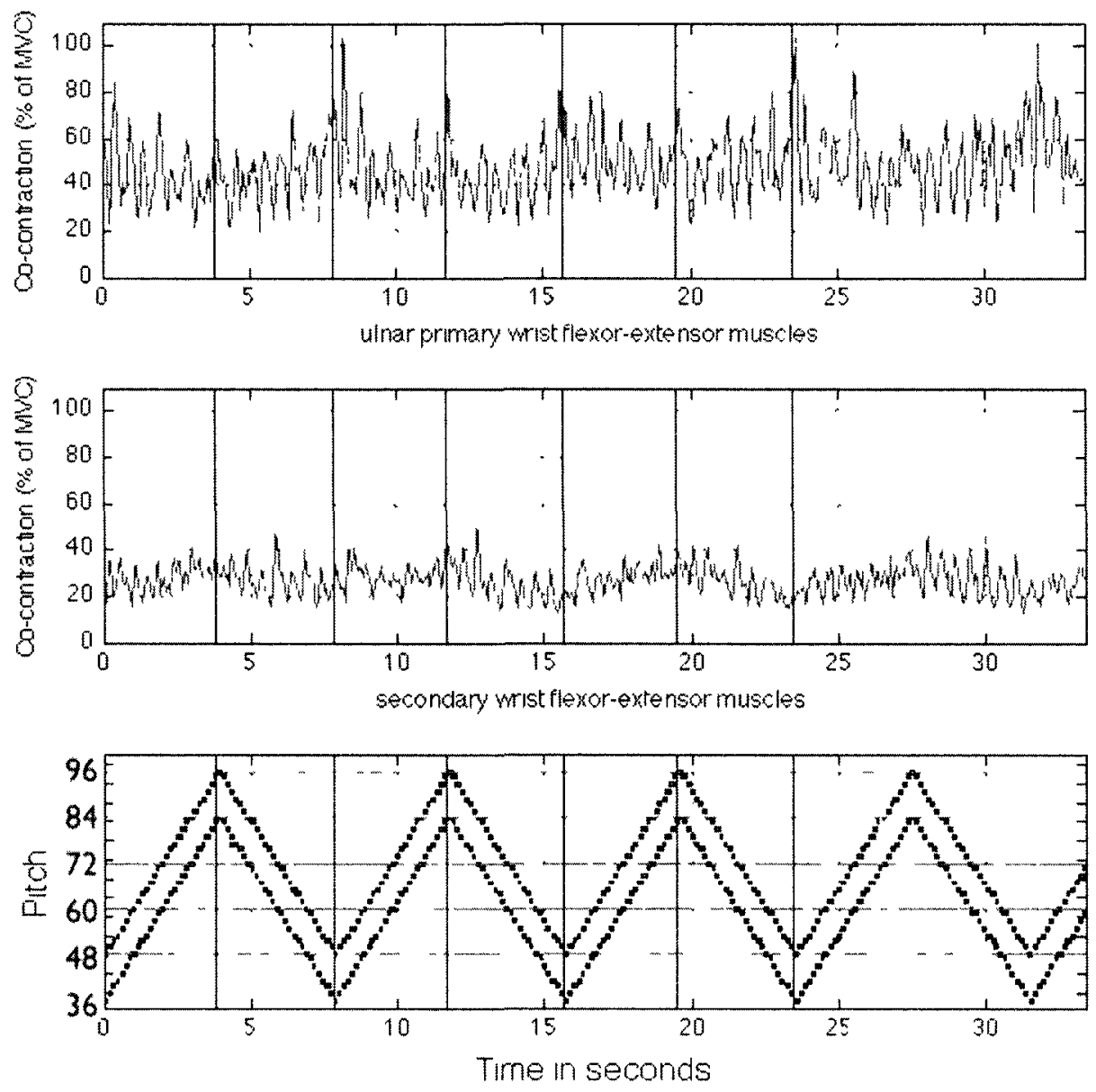

Figure H.16: Participant E010's results: the upper plot shows levels of muscular co-contraction, expressed as a percentage of maximal voluntary contraction, while playing the $\mathrm{C}$ major scale in parallel motion, ascending and descending. The corresponding MIDI data (note on, note off) are shown on the lower plot. Participant E010 played the scale, both ascending and descending segments, an additional time, and began to play the scale for a fifth time before being told to stop. The data collected during the playing of the additional octaves are shown in the figure; however, only the first three octaves are used in the analyses of the scale. 
Appendix I

Participants' Mean Active Muscle

Stiffnesses and Mean Co-contraction

Levels for Pooled Ascending and Pooled

Descending Segments of the C Major

Scale 
Table I.1: Mean active muscle stiffnesses for [pooled] ascending and [pooled] descending segments of the $\mathrm{C}$ major scale. Results are expressed as a percentage of the MVC. During participant E006's execution of the scale, there was interference between the wire connecting the FCU sensor to the main amplifier and the electrodes for approximately $8.5 \mathrm{~s}$. As a result, the data collected during this time do not accurately indicate the level of active muscle stiffness. The contaminated data were left out of the calculation of the mean; this is indicated by $\S$.

\begin{tabular}{|c|c|c|c|c|c|c|c|c|c|}
\hline & \multicolumn{8}{|c|}{ Mean Active Muscle Stiffness (as a \% of MVC) } \\
\hline & & \multicolumn{4}{|c|}{ Ascending } & \multicolumn{4}{|c|}{ Descending } \\
\hline & & FCU & ECU & FDS & EDC & FCU & ECU & FDS & EDC \\
\hline \multirow{8}{*}{ 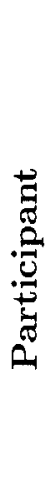 } & E002 & 29.12 & 54.22 & 17.11 & 33.14 & 30.24 & 49.26 & 15.66 & 36.76 \\
\hline & E003 & 29.02 & 50.85 & 32.96 & 38.87 & 28.13 & 48.85 & 37.06 & 32.75 \\
\hline & E004 & 33.69 & 34.06 & 14.87 & 31.70 & 33.63 & 35.74 & 12.33 & 30.91 \\
\hline & E005 & 35.14 & 44.04 & 46.14 & 35.65 & 38.35 & 34.81 & 35.42 & 31.08 \\
\hline & E006 & $31.79 \S$ & 28.93 & 18.86 & 19.30 & $34.68 \S$ & 24.52 & 15.36 & 15.76 \\
\hline & E007 & 44.54 & 26.93 & 42.44 & 12.63 & 40.16 & 29.64 & 35.83 & 14.17 \\
\hline & E009 & 15.65 & 43.95 & 38.75 & 36.49 & 15.29 & 52.36 & 33.93 & 41.32 \\
\hline & E010 & 53.54 & 45.67 & 46.10 & 19.15 & 60.42 & 39.54 & 40.57 & 18.97 \\
\hline
\end{tabular}


Table I.2: Mean co-contraction levels for [pooled] ascending and [pooled] descending segments of the $\mathrm{C}$ major scale. Results are expressed as a percentage of the MVC. During participant E006's execution of the scale, there was interference between the wire connecting the FCU sensor to the main amplifier and the electrodes for approximately $8.5 \mathrm{~s}$. As a result, the data collected during this time do not accurately indicate the level of active muscle stiffness, and thus co-contraction could not be calculated accurately for this time either. The contaminated data were left out of the calculation of the mean $\mathrm{CC}^{\circ}$; this is indicated by $\S$.

\begin{tabular}{|c|c|c|c|c|c|}
\hline & \multicolumn{4}{|c|}{ Mean Co-contraction (as a \% of MVC) } \\
\hline & & \multicolumn{2}{|c|}{ Ascending } & \multicolumn{2}{|c|}{ Descending } \\
\hline & & CC1 & $\mathrm{CC} 2$ & CC1 & $\mathrm{CC} 2$ \\
\hline \multirow{8}{*}{ 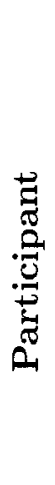 } & E002 & 39.34 & 23.43 & 38.24 & 23.80 \\
\hline & E003 & 37.59 & 34.48 & 35.85 & 32.04 \\
\hline & E004 & 32.47 & 21.41 & 33.35 & 19.22 \\
\hline & E005 & 37.79 & 39.98 & 35.15 & 32.25 \\
\hline & E006 & $28.74 \S$ & 18.51 & $28.17 \S$ & 15.06 \\
\hline & E007 & 34.11 & 22.30 & 34.21 & 22.14 \\
\hline & E009 & 24.84 & 35.58 & 27.50 & 35.68 \\
\hline & E010 & 47.49 & 28.31 & 46.96 & 26.24 \\
\hline
\end{tabular}


Appendix $\mathbf{J}$

Participants' Mean Active Muscle Stiffnesses and Mean Co-contraction Levels for Ascending (A1, A2, A3) and Descending (D1, D2, D3) Segments of the C Major Scale 
Table J.1: Mean active muscle stiffnesses in segments A1, A2 and $A 3$ of the C major scale. Results are expressed as a percentage of the MVC. During participant E006's execution of the scale, there was interference between the wire connecting the FCU sensor to the main amplifier and the electrodes for approximately $8.5 \mathrm{~s}$. As a result, the data collected during this time do not accurately indicate the level of active muscle stiffness. The contaminated data were left out of the calculation of the mean; this is indicated by $\S$. In the case of the first ascending segment, all FCU data were contaminated, thus there is no mean for this segment.

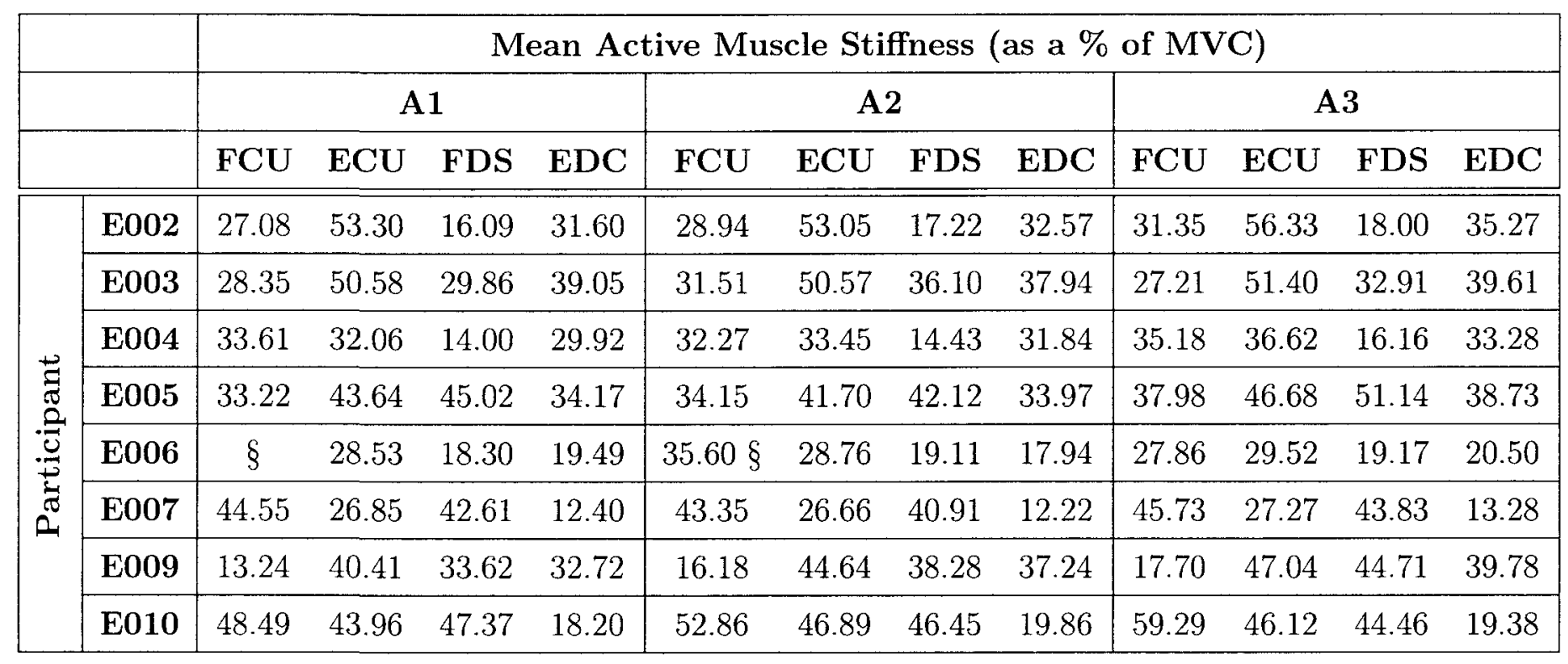


Table J.2: Mean active muscle stiffnesses in segments $D 1, D 2$, and $D 3$ of the C major scale. Results are expressed as a percentage of the MVC. During participant E006's execution of the scale, there was interference between the wire connecting the FCU sensor to the main amplifier and the electrodes for approximately $8.5 \mathrm{~s}$. As a result, the data collected during this time do not accurately indicate the level of active muscle stiffness. The contaminated data were left out of the calculation of the mean; this is indicated by $\S$. In the case of the first descending segment, all FCU data were contaminated, thus there is no mean for this segment.

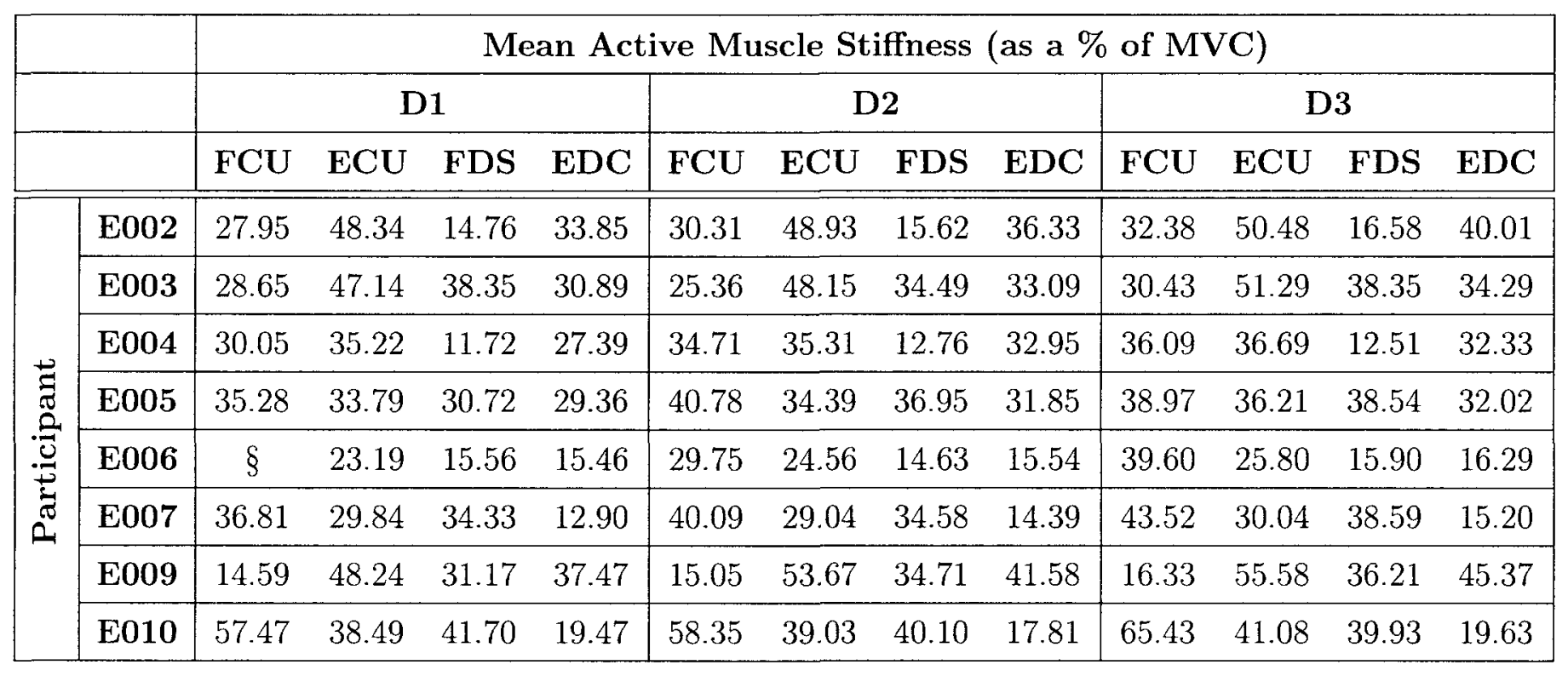


Table J.3: Mean co-contraction levels in segments $A 1, A 2$ and $A 3$ of the $\mathrm{C}$ major scale. Results are expressed as a percentage of the MVC. During participant E006's execution of the scale, there was interference between the wire connecting the FCU sensor to the main amplifier and the electrodes for approximately 8.5 $\mathrm{s}$. As a result, the data collected during this time do not accurately indicate the level of active muscle stiffness, and thus co-contraction could not be calculated accurately for this time either. The contaminated data were left out of the calculation of the mean co-contraction; this is indicated by $\S$. In the case of the first ascending segment, all FCU data were contaminated, and consequently there is no mean $\mathrm{CC} 1$ for this segment.

\begin{tabular}{|c|c|c|c|c|c|c|c|}
\hline & \multicolumn{6}{|c|}{ Mean Co-contraction (as a \% of MVC) } \\
\hline & & \multicolumn{2}{|c|}{ A1 } & \multicolumn{2}{|c|}{ A2 } & \multicolumn{2}{|c|}{ A3 } \\
\hline & & $\mathrm{CC} 1$ & $\mathrm{CC} 2$ & $\mathrm{CC} 1$ & $\mathrm{CC} 2$ & $\mathrm{CC} 1$ & $\mathrm{CC} 2$ \\
\hline \multirow{8}{*}{ 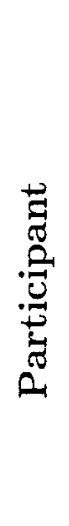 } & E002 & 37.67 & 22.21 & 38.83 & 23.34 & 41.54 & 24.75 \\
\hline & E003 & 37.25 & 32.95 & 38.97 & 35.61 & 36.56 & 34.87 \\
\hline & E004 & 31.53 & 20.20 & 31.37 & 21.15 & 34.46 & 22.84 \\
\hline & E005 & 36.70 & 38.73 & 36.29 & 37.21 & 40.31 & 43.90 \\
\hline & E006 & $\S$ & 18.21 & $29.81 \S$ & 18.11 & 27.64 & 19.22 \\
\hline & E007 & 34.01 & 22.05 & 33.41 & 21.66 & 34.92 & 23.21 \\
\hline & E009 & 21.91 & 31.43 & 25.33 & 35.68 & 27.48 & 39.91 \\
\hline & E010 & 44.26 & 27.96 & 47.58 & 28.89 & 50.62 & 28.08 \\
\hline
\end{tabular}


Table J.4: Mean co-contraction levels in segments D1, D2, and D3 of the C major scale. Results are expressed as a percentage of the MVC. During participant E006's execution of the scale, there was interference between the wire connecting the FCU sensor to the main amplifier and the electrodes for approximately 8.5 s. As a result, the data collected during this time do not accurately indicate the level of active muscle stiffness, and thus co-contraction could not be calculated accurately for this time either. The contaminated data were left out of the calculation of the mean co-contraction; this is indicated by $\S$. In the case of the first descending segment, all FCU data were contaminated, and consequently there is no mean $\mathrm{CC} 1$ for this segment.

\begin{tabular}{|c|c|c|c|c|c|c|c|}
\hline & \multicolumn{6}{|c|}{ Mean Co-contraction (as a \% of MVC) } \\
\hline & & \multicolumn{2}{|c|}{ D1 } & \multicolumn{2}{|c|}{ D2 } & \multicolumn{2}{|c|}{ D3 } \\
\hline & & CC1 & $\mathrm{CC} 2$ & $\mathrm{CC} 1$ & $\mathrm{CC} 2$ & $\mathrm{CC} 1$ & $\mathrm{CC} 2$ \\
\hline \multirow{8}{*}{ 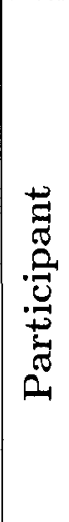 } & E002 & 36.43 & 22.16 & 38.22 & 23.68 & 40.03 & 25.51 \\
\hline & E003 & 35.85 & 31.60 & 33.94 & 31.14 & 37.79 & 33.39 \\
\hline & E004 & 31.42 & 17.67 & 33.51 & 20.21 & 35.08 & 19.74 \\
\hline & E005 & 33.03 & 29.25 & 35.89 & 33.31 & 36.50 & 34.14 \\
\hline & E006 & $\S$ & 14.93 & 25.96 & 14.68 & 30.38 & 15.58 \\
\hline & E007 & 32.93 & 20.67 & 33.77 & 21.83 & 35.92 & 23.89 \\
\hline & E009 & 25.71 & 32.55 & 27.61 & 36.25 & 29.40 & 38.59 \\
\hline & E010 & 45.37 & 26.85 & 45.48 & 25.33 & 50.04 & 26.55 \\
\hline
\end{tabular}




\begin{abstract}
Appendix K
Plots of Participants' Active Muscle Stiffnesses and MIDI Data While Playing Triad Exercises TR1 and TR2
\end{abstract}



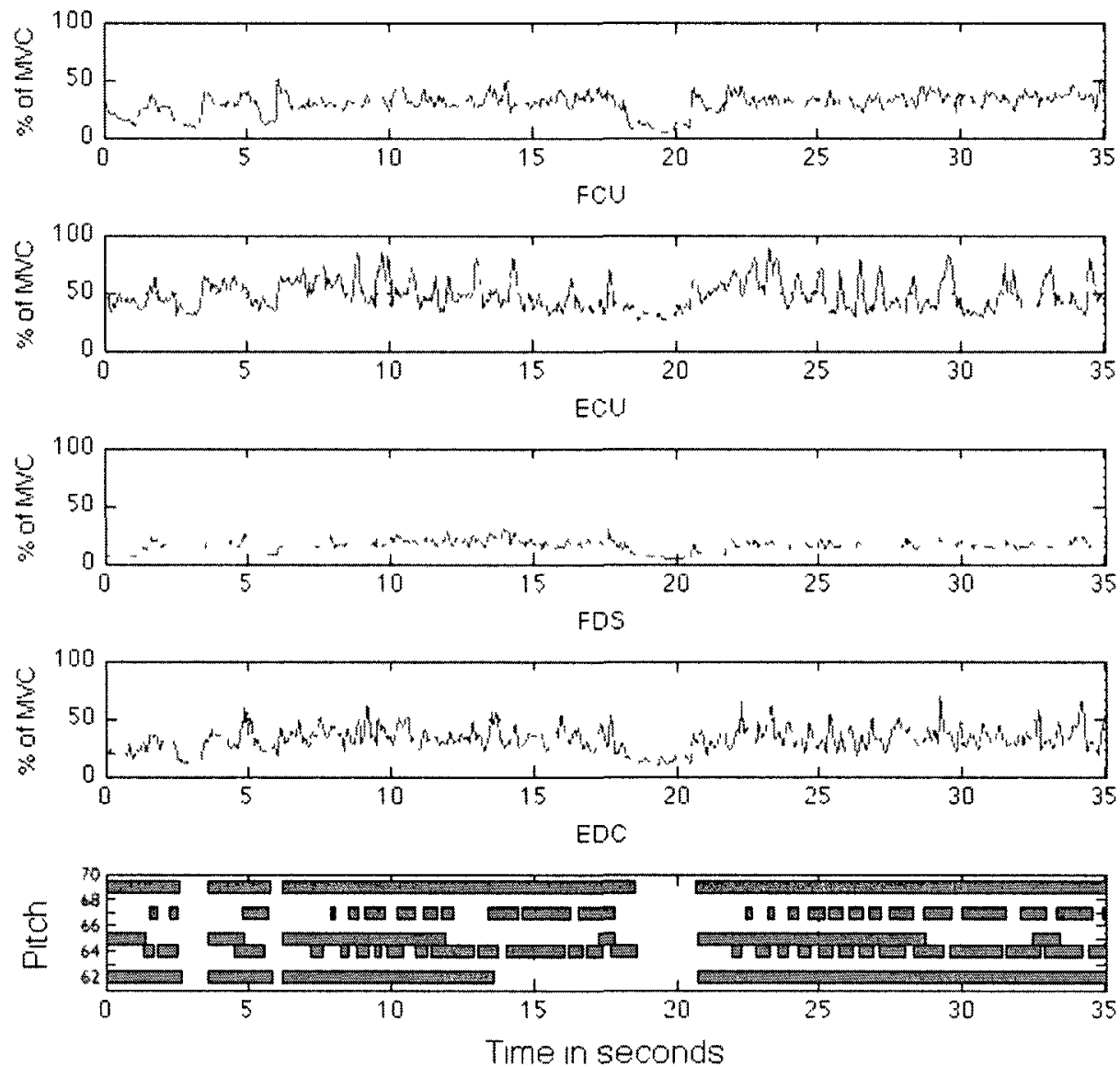

Figure K.1: Participant E002's EMG results: the upper plots show active muscle stiffness, expressed as a percentage of maximal voluntary contraction, while playing the first triad exercise (TR1) for four muscles of the right forearm: the FCU, the ECU, the FDS and the EDC. The corresponding MIDI data (note on, note off) are shown on the lower plot. This participant lifted their fingers $>10 \%$ of the time, and thus their results are not valid for further analysis. 

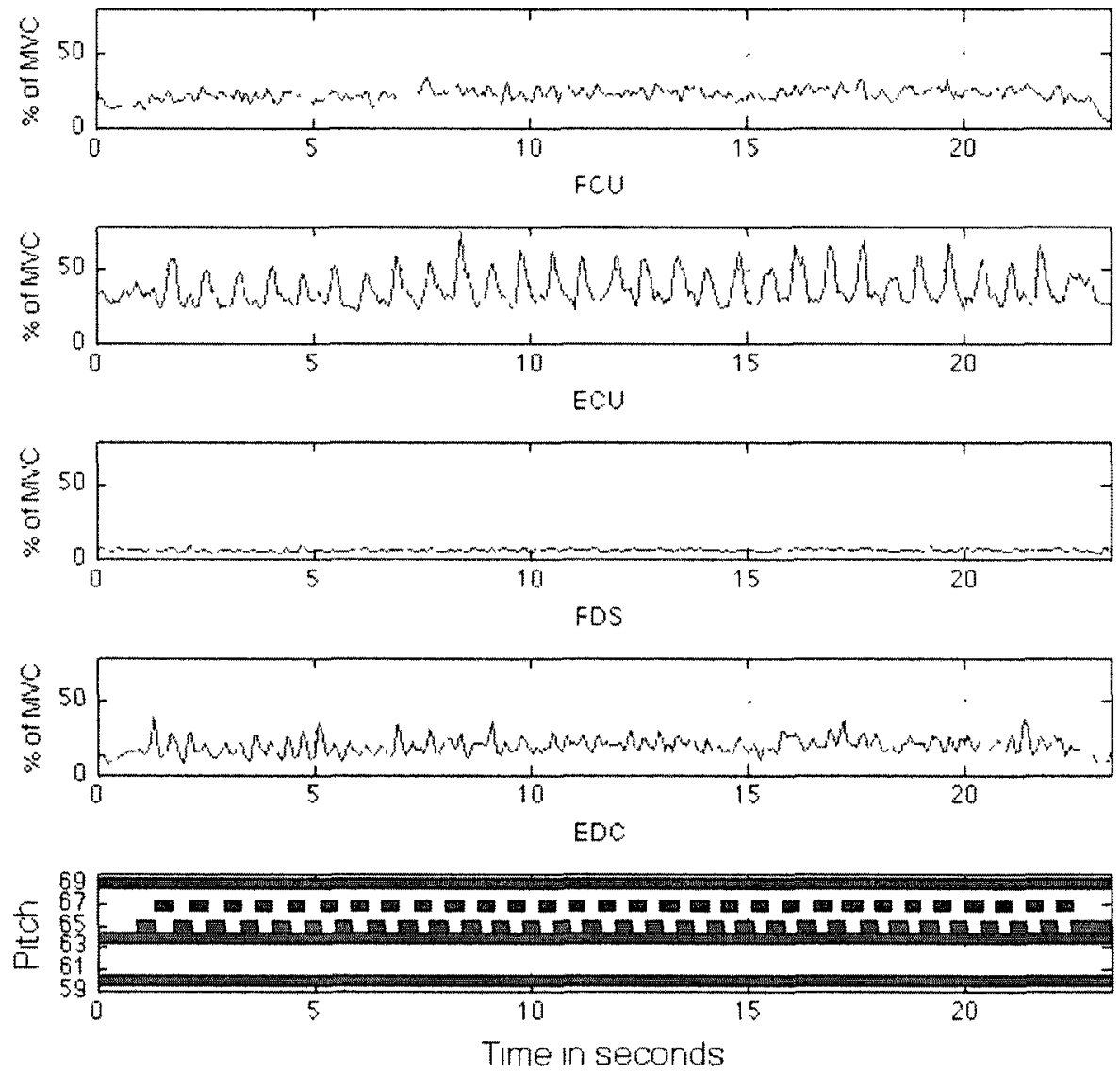

Figure K.2: Participant E002's EMG results: the upper plots show active muscle stiffness, expressed as a percentage of maximal voluntary contraction, while playing the second triad exercise (TR2) for four muscles of the right forearm: the FCU, the ECU, the FDS and the EDC. The corresponding MIDI data (note on, note off) are shown on the lower plot. 

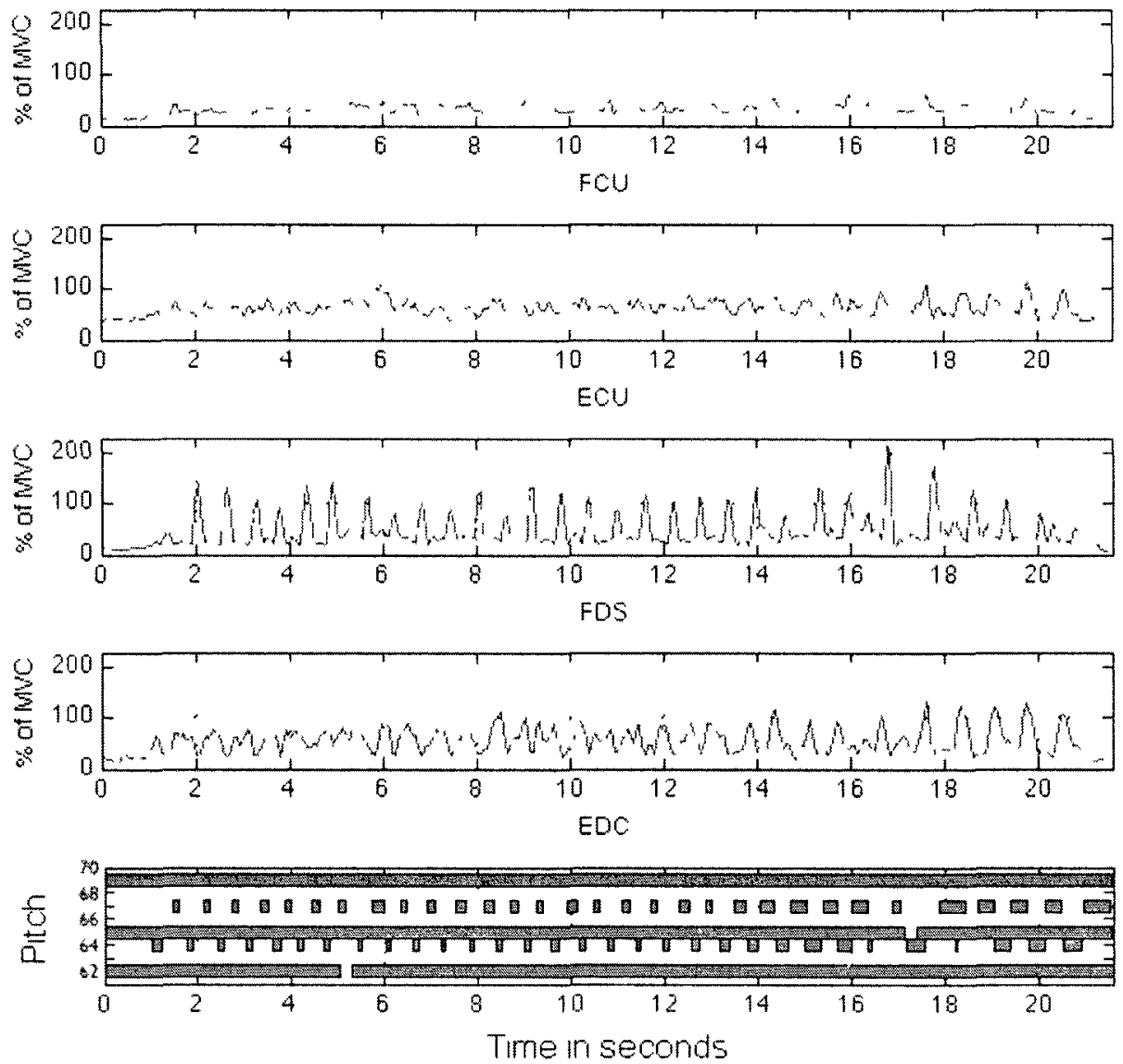

Figure K.3: Participant E003's EMG results: the upper plots show active muscle stiffness, expressed as a percentage of maximal voluntary contraction, whlle playing the first triad exercise (TR1) for four muscles of the right forearm: the FCU, the ECU, the FDS and the EDC. The corresponding MIDI data (note on, note off) are shown on the lower plot. 

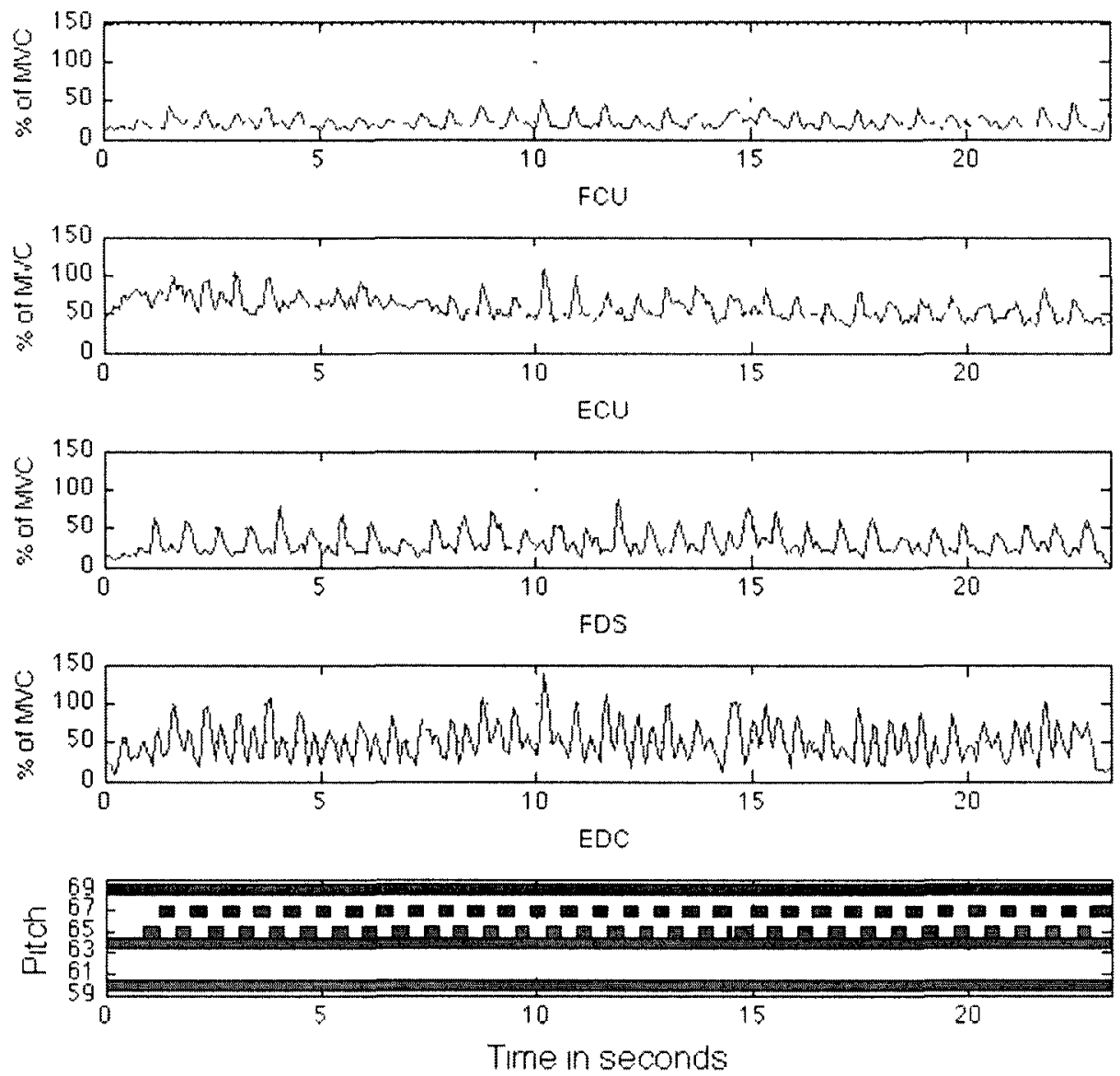

Figure K.4: Participant E003's EMG results: the upper plots show active muscle stiffness, expressed as a percentage of maximal voluntary contraction, while playing the second triad exercise (TR2) for four muscles of the right forearm: the FCU, the ECU, the FDS and the EDC. The corresponding MIDI data (note on, note off) are shown on the lower plot. 

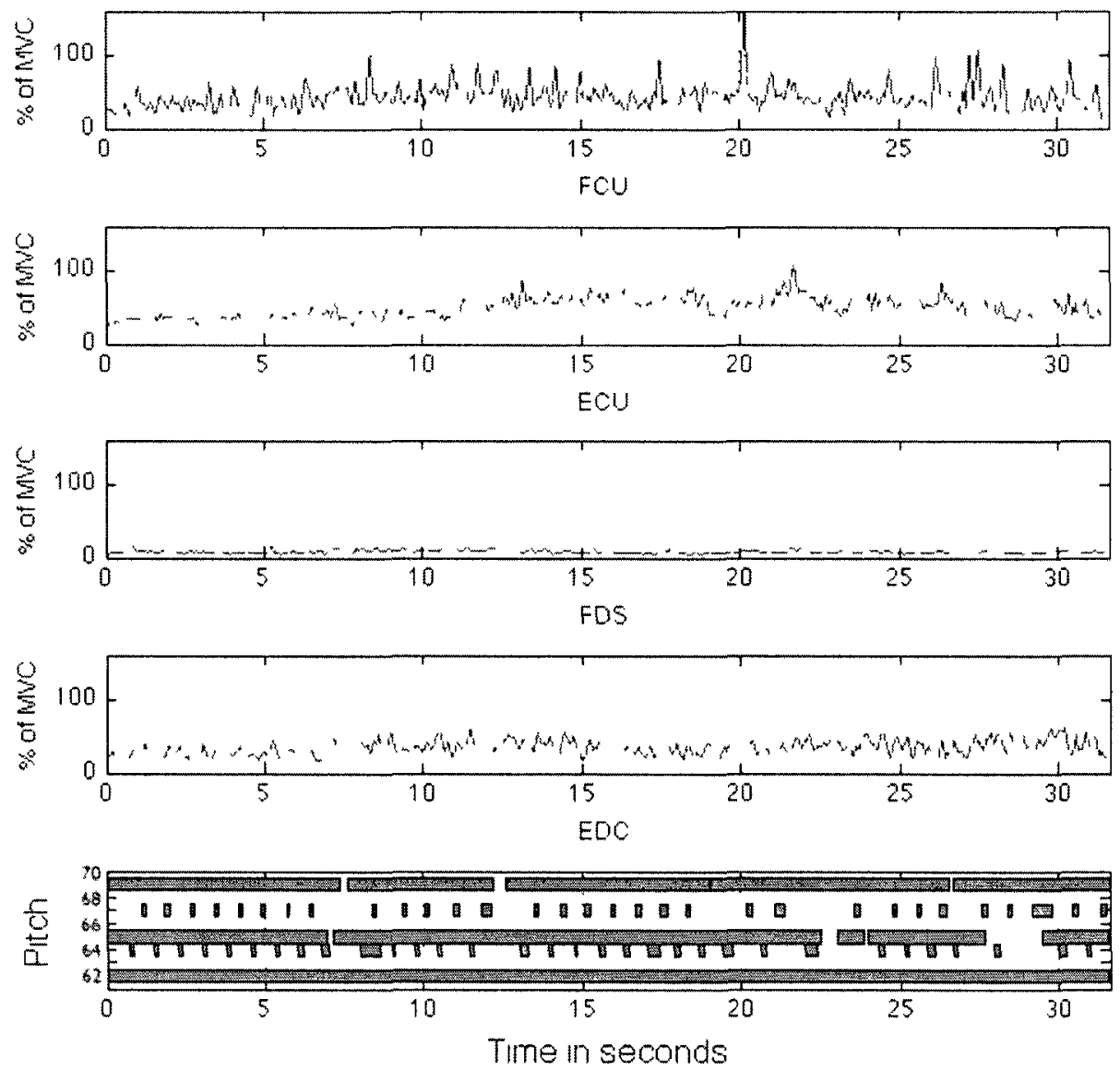

Figure K.5: Participant E004's EMG results. the upper plots show active muscle stiffness, expressed as a percentage of maximal voluntary contraction, while playing the first triad exercise (TR1) for four muscles of the right forearm the FCU, the ECU, the FDS and the EDC The corresponding MIDI data (note on, note off) are shown on the lower plot 

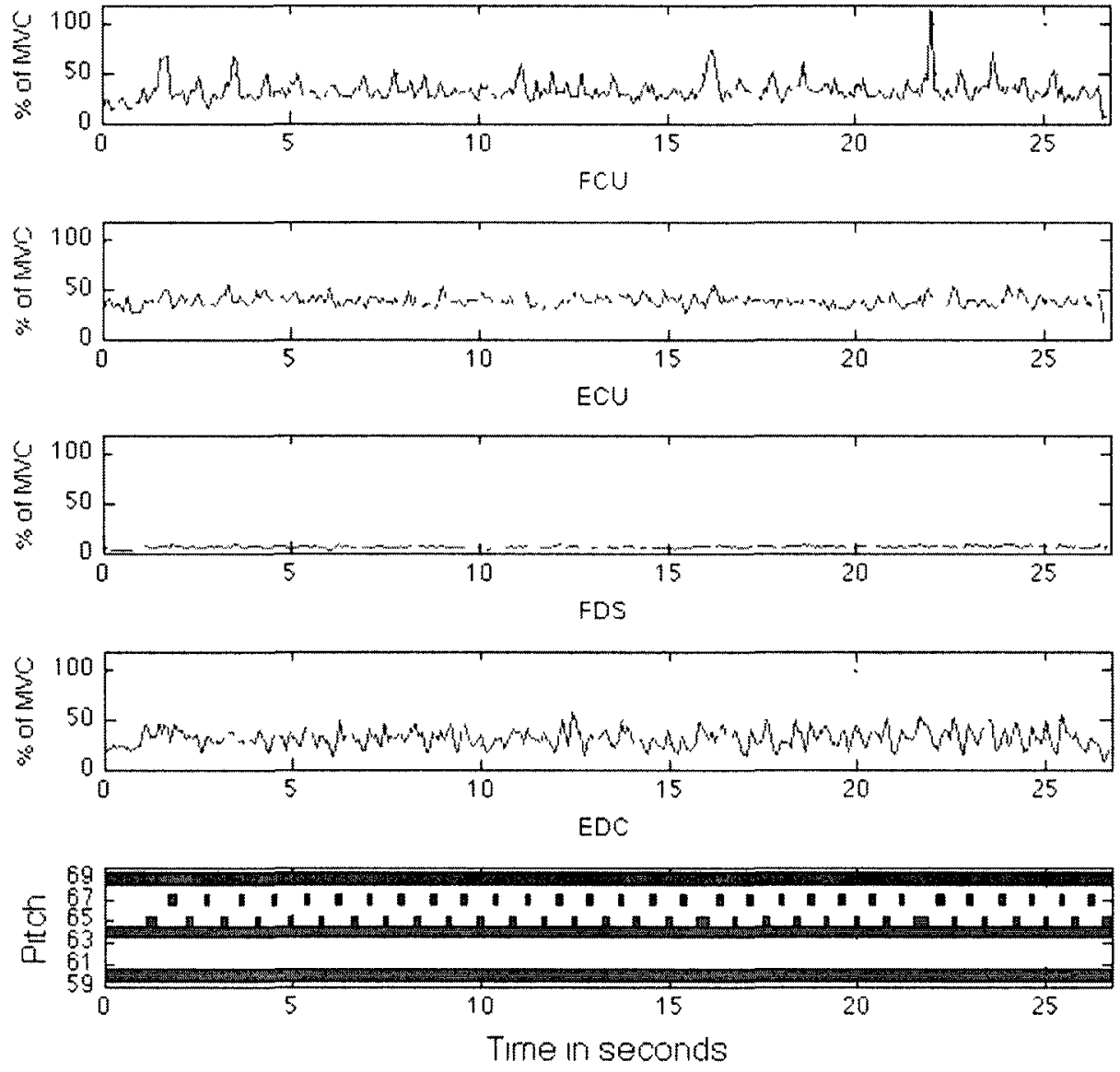

Figure K.6: Participant E004's EMG results: the upper plots show active muscle stiffness, expressed as a percentage of maximal voluntary contraction, while playing the second triad exercise (TR2) for four muscles of the right forearm. the FCU, the ECU, the FDS and the EDC. The corresponding MIDI data (note on, note off) are shown on the lower plot. 

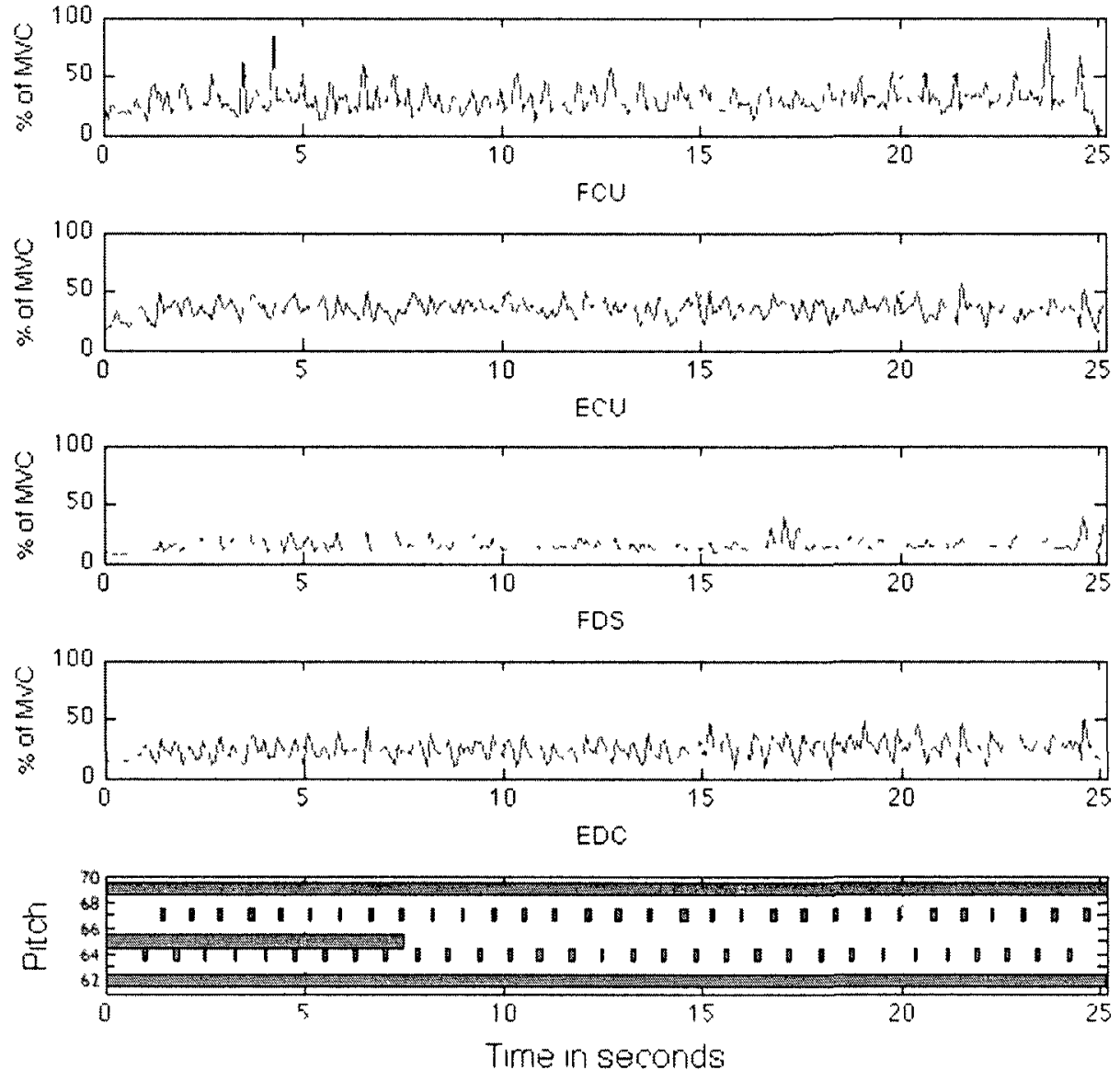

Figure K.7: Participant E005's EMG results: the upper plots show active muscle stiffness, expressed as a percentage of maximal voluntary contraction, while playing the first triad exercise (TR1) for four muscles of the right forearm: the FCU, the ECU, the FDS and the EDC. The corresponding MIDI data (note on, note off) are shown on the lower plot. This participant lifted fr $3>10 \%$ of the tıme, and thus their results are not valid for further analysis. 

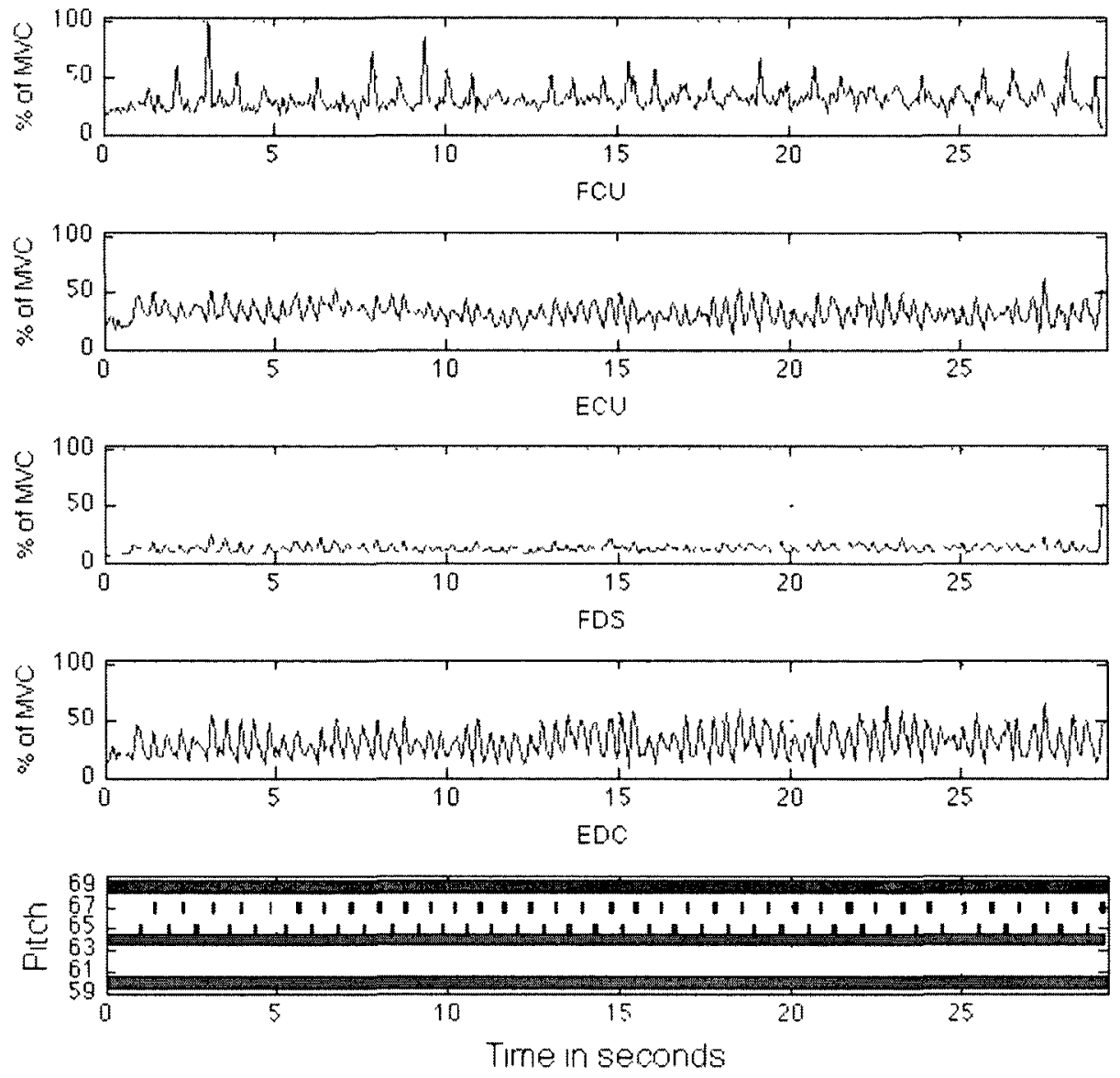

Figure K.8: Participant E005's EMG results: the upper plots show active muscle stiffness, expressed as a percentage of maximal voluntary contraction, while playing the second triad exercise (TR2) for four muscles of the right forearm: the FCU, the ECU, the FDS and the EDC. The corresponding MIDI data (note on, note off) are shown on the lower plot. 

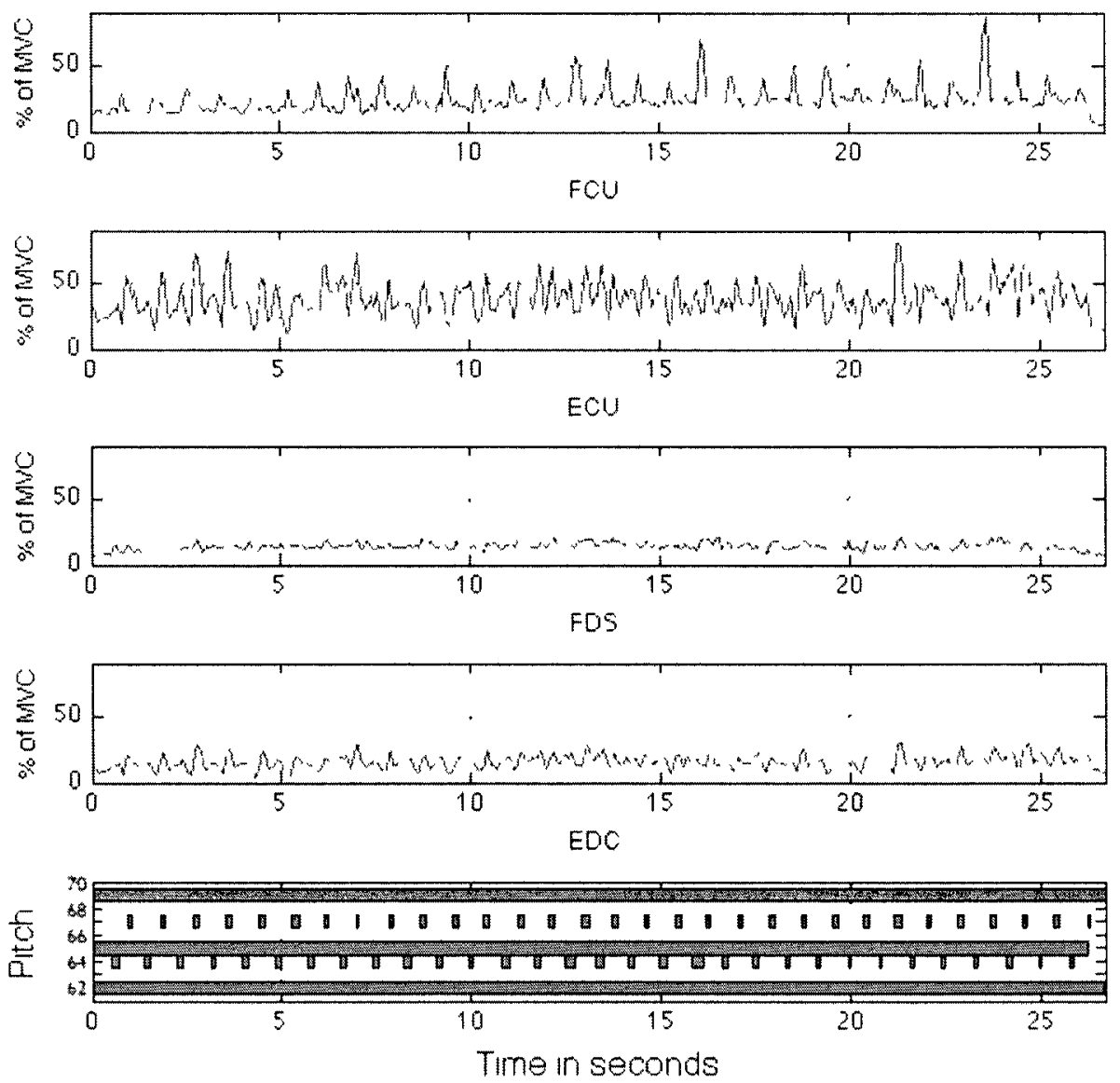

Figure K.9: Participant E006's EMG results: the upper plots show active muscle stiffness, expressed as a percentage of maximal voluntary contraction, while playing the first triad exercise (TR1) for four muscles of the right forearm: the FCU, the ECU, the FDS and the EDC. The corresponding MIDI data (note on, note off) are shown on the lower plot. 

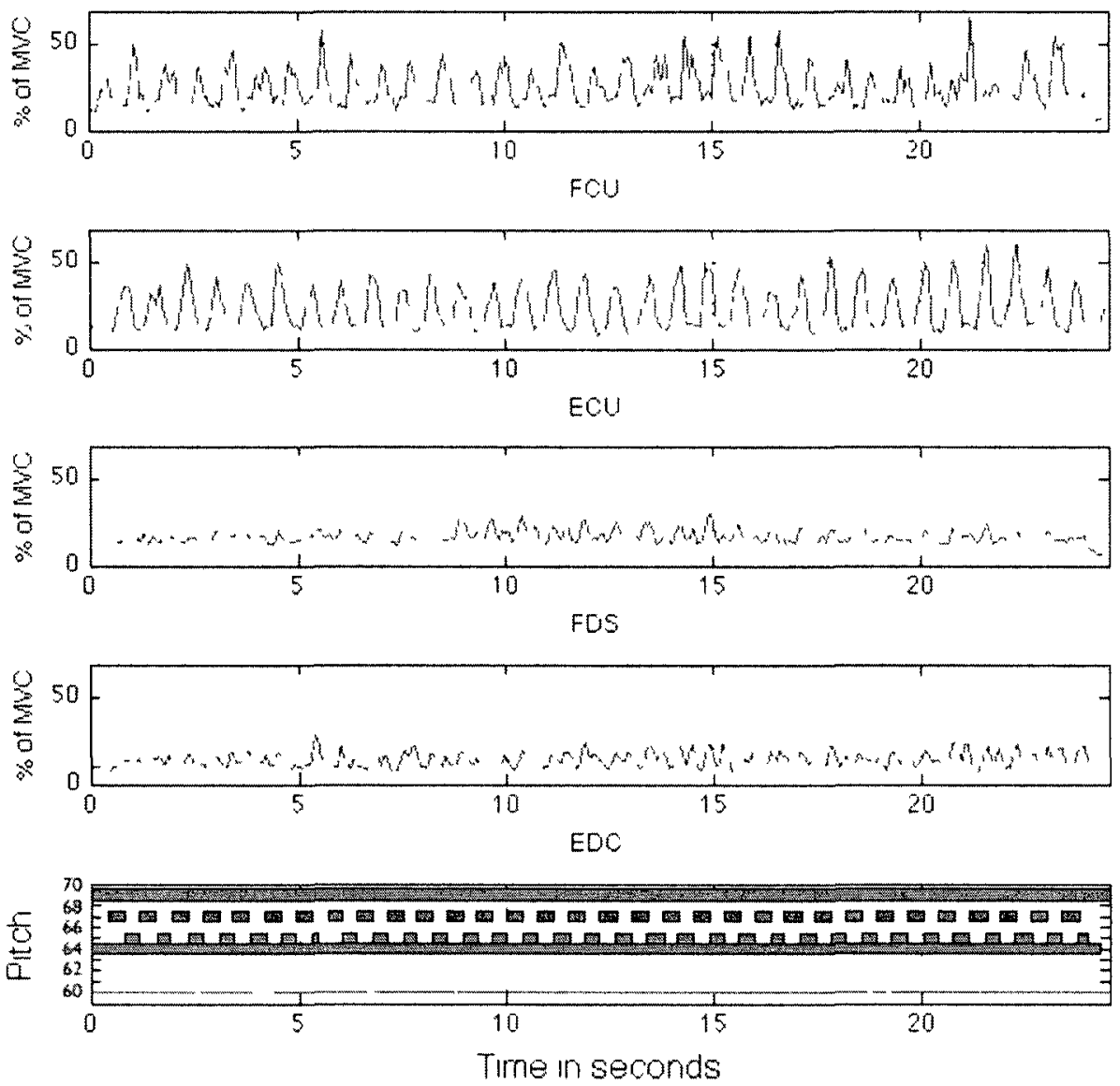

Figure K.10: Participant E006's EMG results: the upper plots show active muscle stiffness, expressed as a percentage of maximal voluntary contraction, while playing the second triad exercise (TR2) for four muscles of the right forearm: the FCU, the ECU, the FDS and the EDC. The corresponding MIDI data (note on, note off) are shown on the lower plot. This participant lifted fr $1>10 \%$ of the time, and thus their results are not valid for further analysis. 

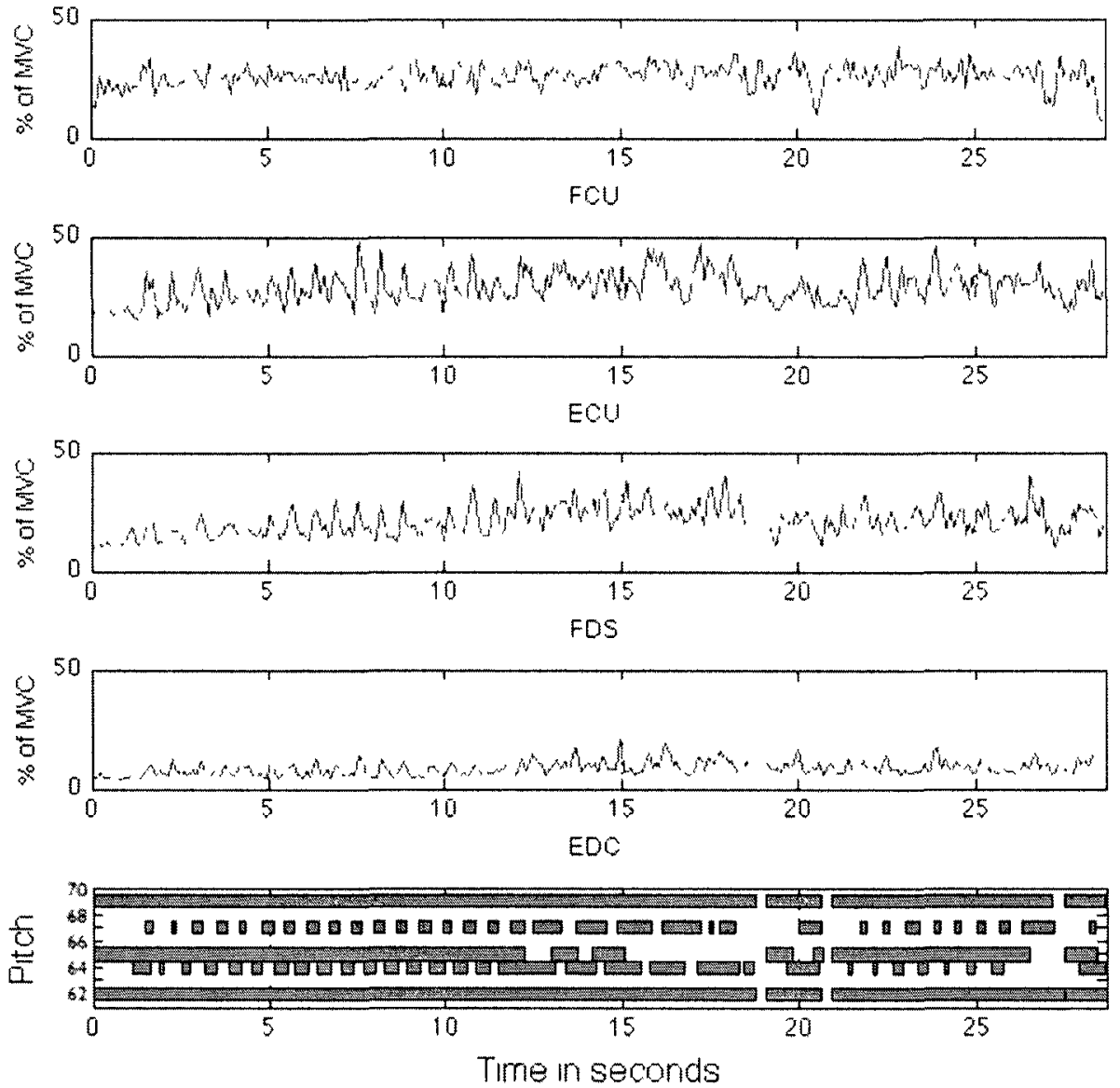

Figure K.11: Participant E007's EMG results: the upper plots show active muscle stiffness, expressed as a percentage of maximal voluntary contraction, while playing the first triad exercise (TR1) for four muscles of the right forearm: the FCU, the ECU, the FDS and the EDC. The corresponding MIDI data (note on, note off) are shown on the lower plot. This participant lifted their fingers $>10 \%$ of the time, and thus their results are not valid for further analysis. 

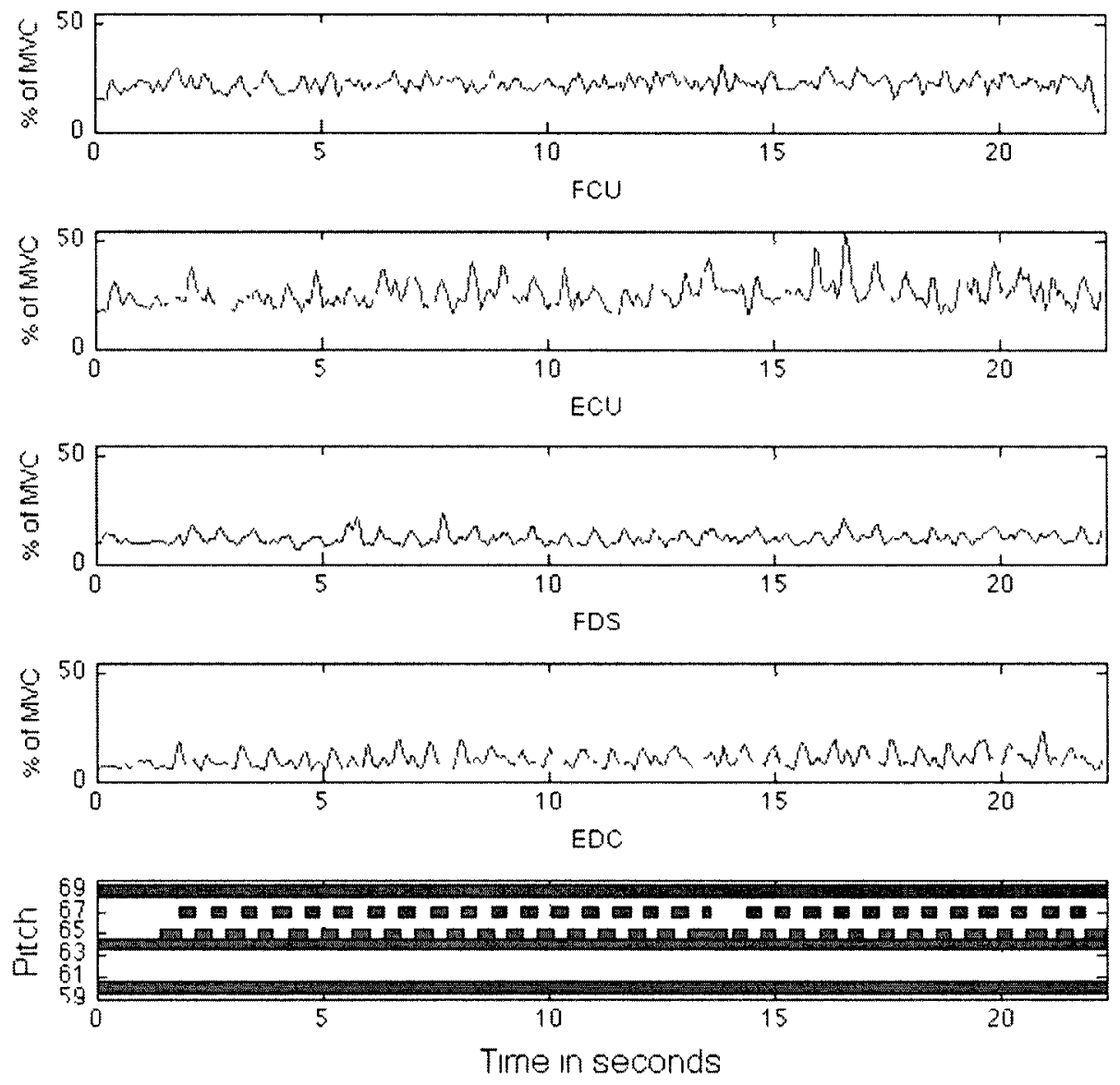

Figure K.12: Participant E007's EMG results: the upper plots show active muscle stiffness, expressed as a percentage of maximal voluntary contraction, while playing the second triad exercise (TR2) for four muscles of the right forearm: the FCU, the ECU, the FDS and the EDC The corresponding MIDI data note on, note off) are shown on the lower plot. 

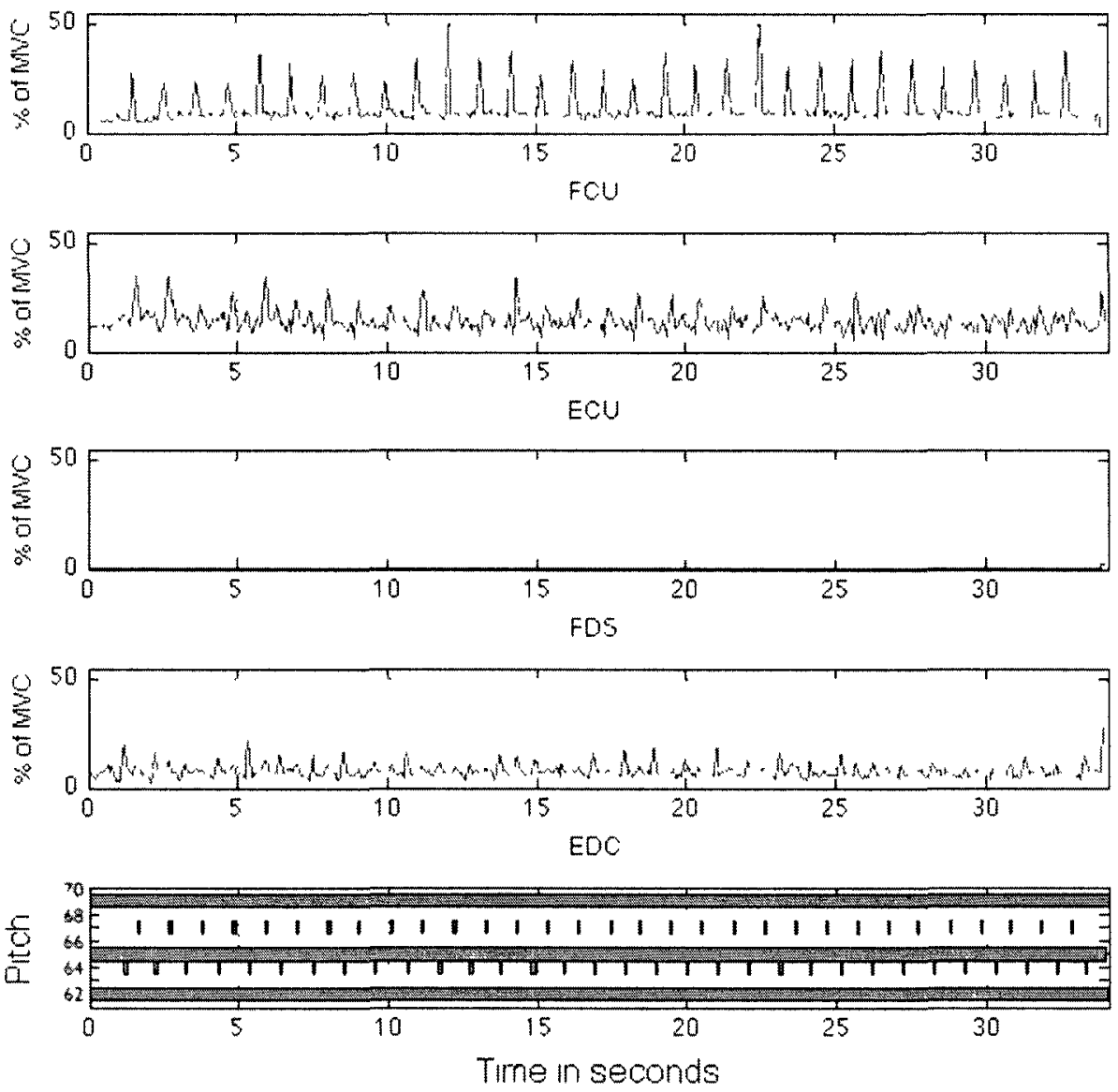

Figure K.13: Participant E008's EMG results: the upper plots show active muscle stiffness, expressed as a percentage of maximal voluntary contraction, while playing the first triad exercise (TR1) for four muscles of the right forearm: the FCU, the ECU, the FDS and the EDC. The corresponding MIDI data (note on, note off) are shown on the lower plot. 

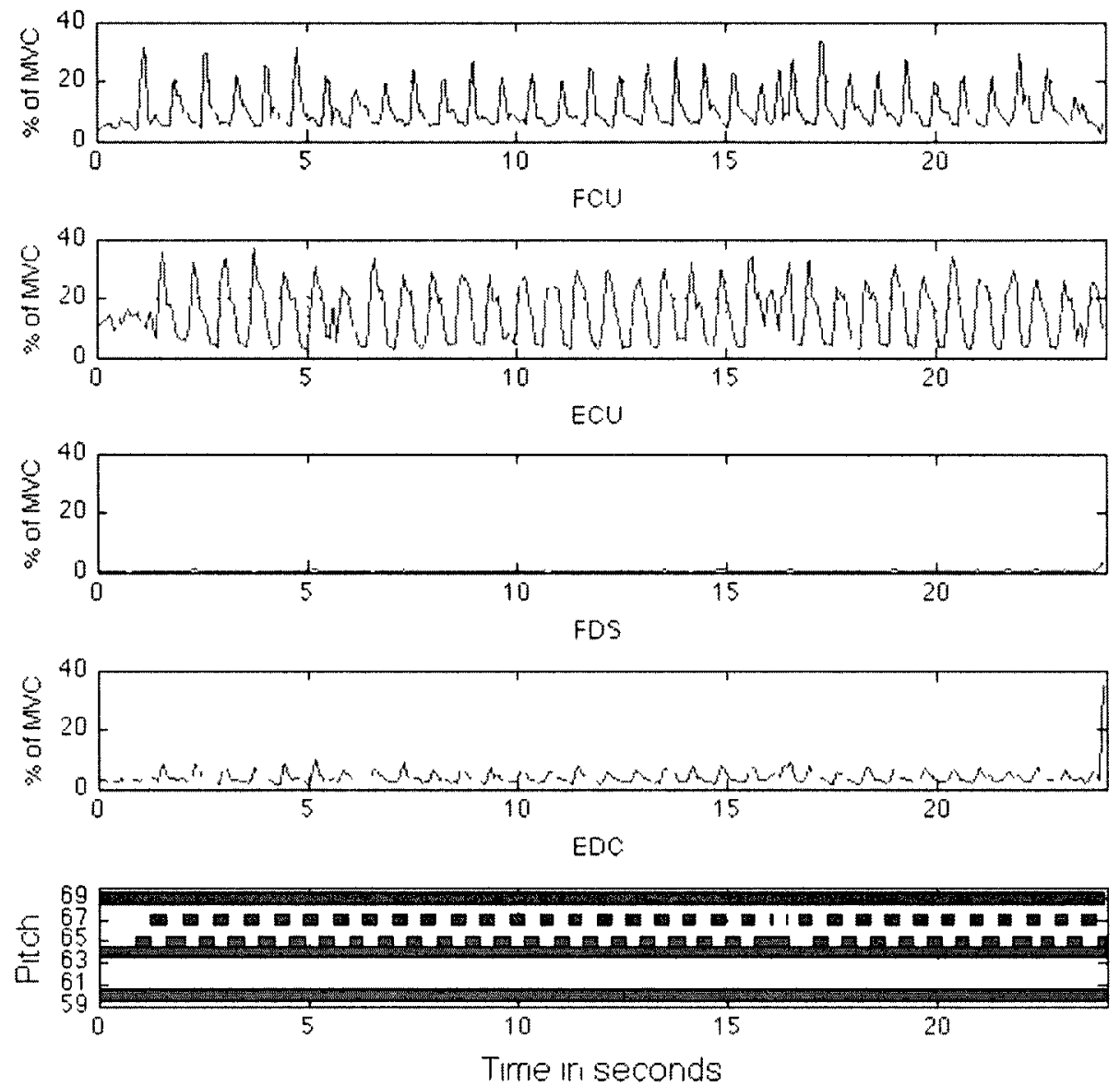

Figure K.14: Participant E008's EMG results. the upper plots show active muscle stiffness, expressed as a percentage of maximal voluntary contraction, while playing the second triad exercise (TR2) for four muscles of the right forearm: the FCU, the ECU, the FDS and the EDC. The corresponding MIDI data (note on, note off) are shown on the lower plot. 

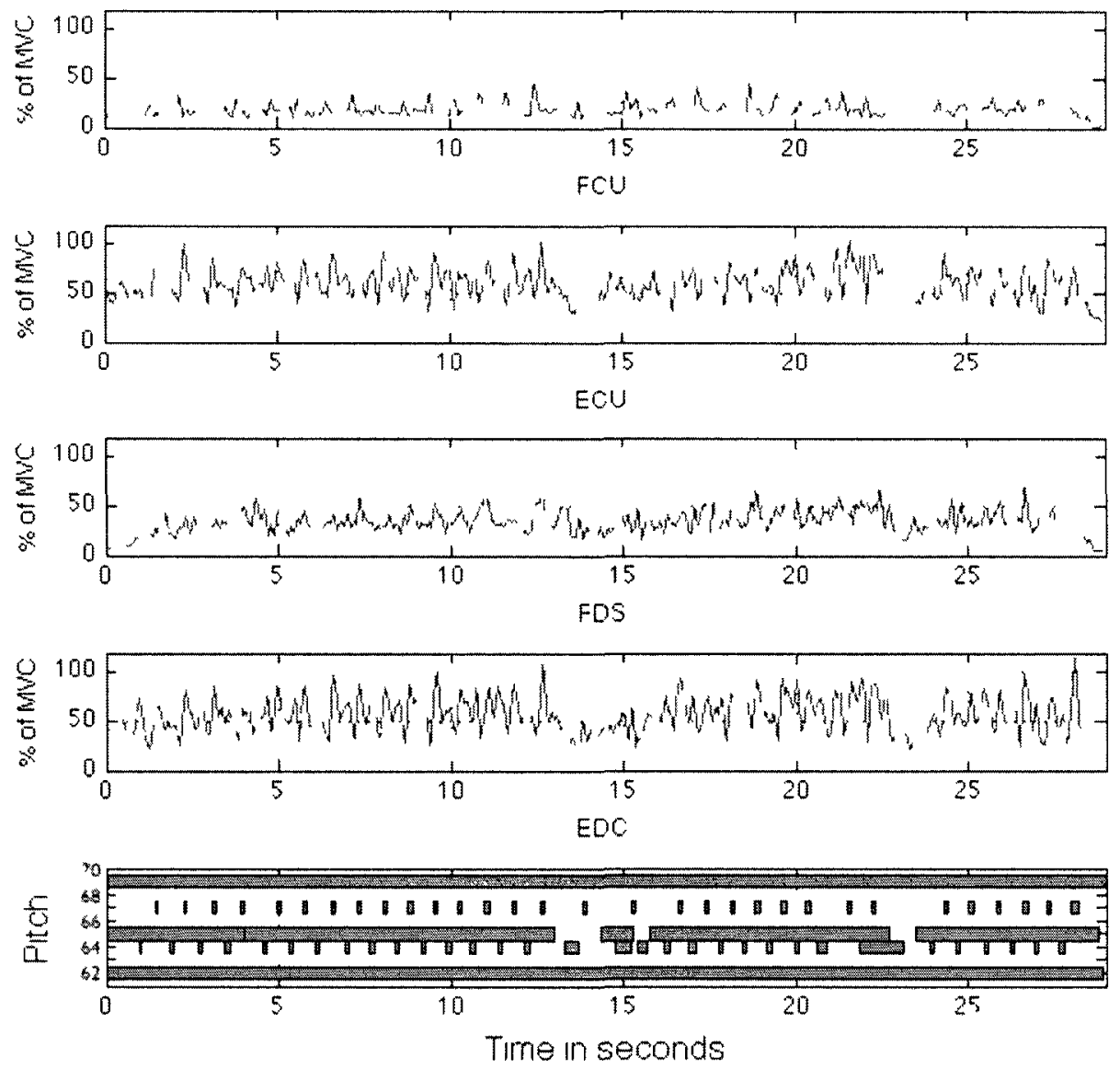

Figure K.15: Partıcıpant E009's EMG results the upper plots show active muscle stıffness, expressed as a percentage of maximal voluntary contraction, while playing the first triad exercise (TR1) for four muscles of the right forearm the FCU, the ECU, the FDS and the EDC The corresponding MIDI data (note on, note off) are shown on the lower plot 

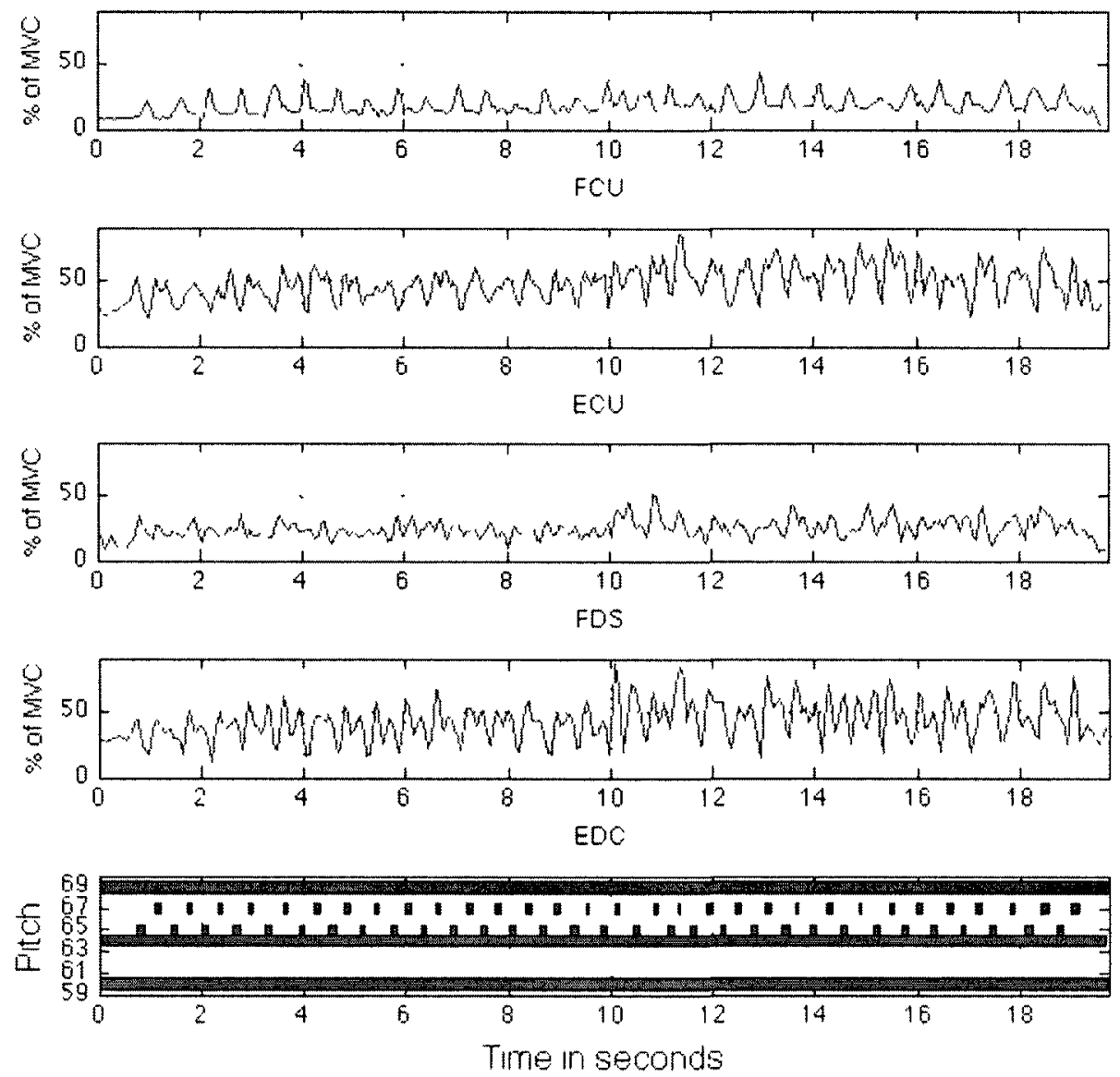

Figure K.16: Participant E009's EMG results: the upper plots show active muscle stiffness, expressed as a percentage of maximal voluntary contraction, while playing the second triad exercise (TR2) for four muscles of the right forearm: the FCU, the ECU, the FDS and the EDC. The corresponding MIDI data (note on, note off) are shown on the lower plot. 

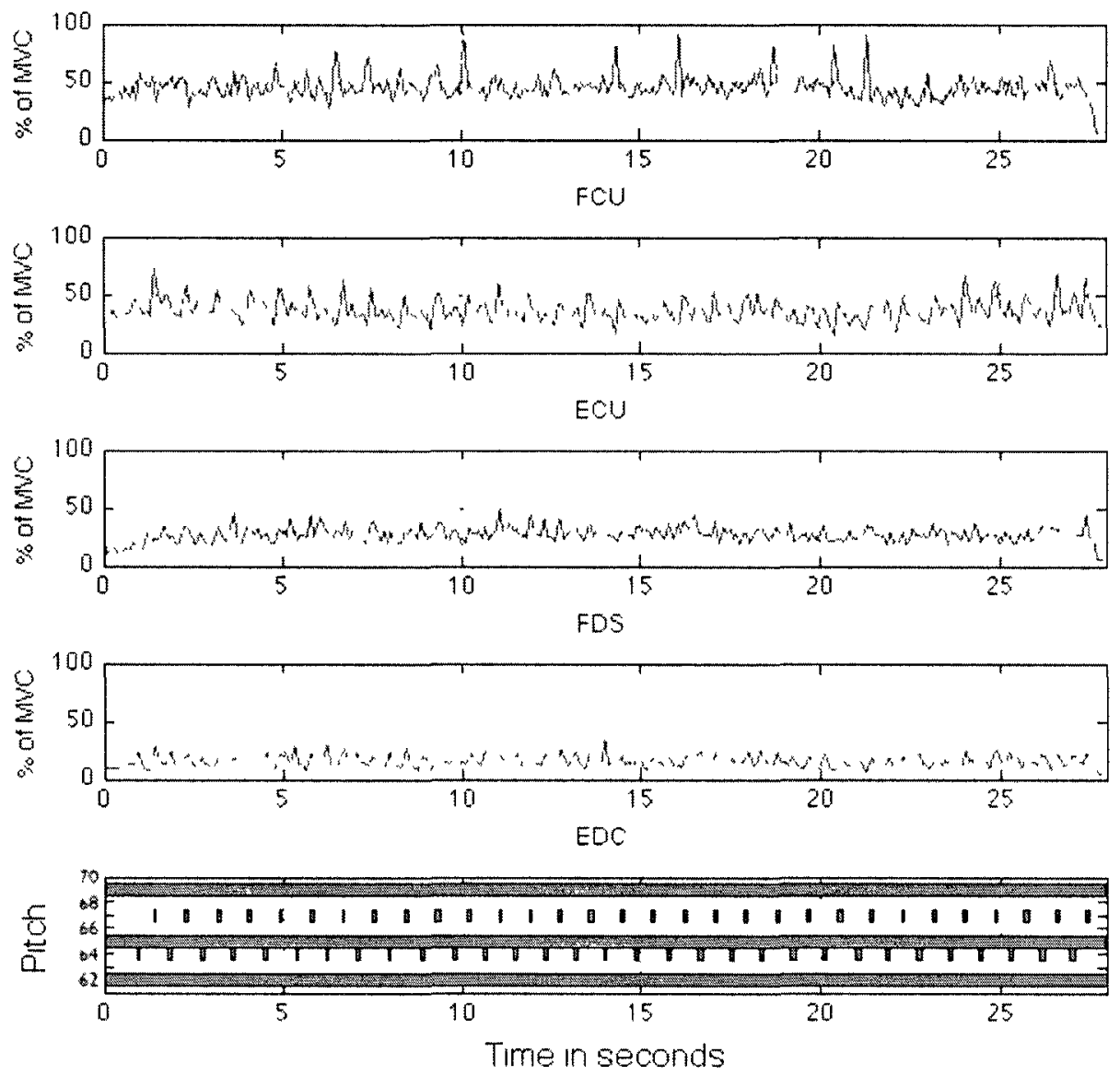

Figure K.17: Participant E010's EMG results: the upper plots show active muscle stiffness, expressed as a percentage of maximal voluntary contraction, while playing the first triad exercise (TR1) for four muscles of the right forearm: the FCU, the ECU, the FDS and the EDC. The corresponding MIDI data (note on, note off) are shown on the lower plot. 

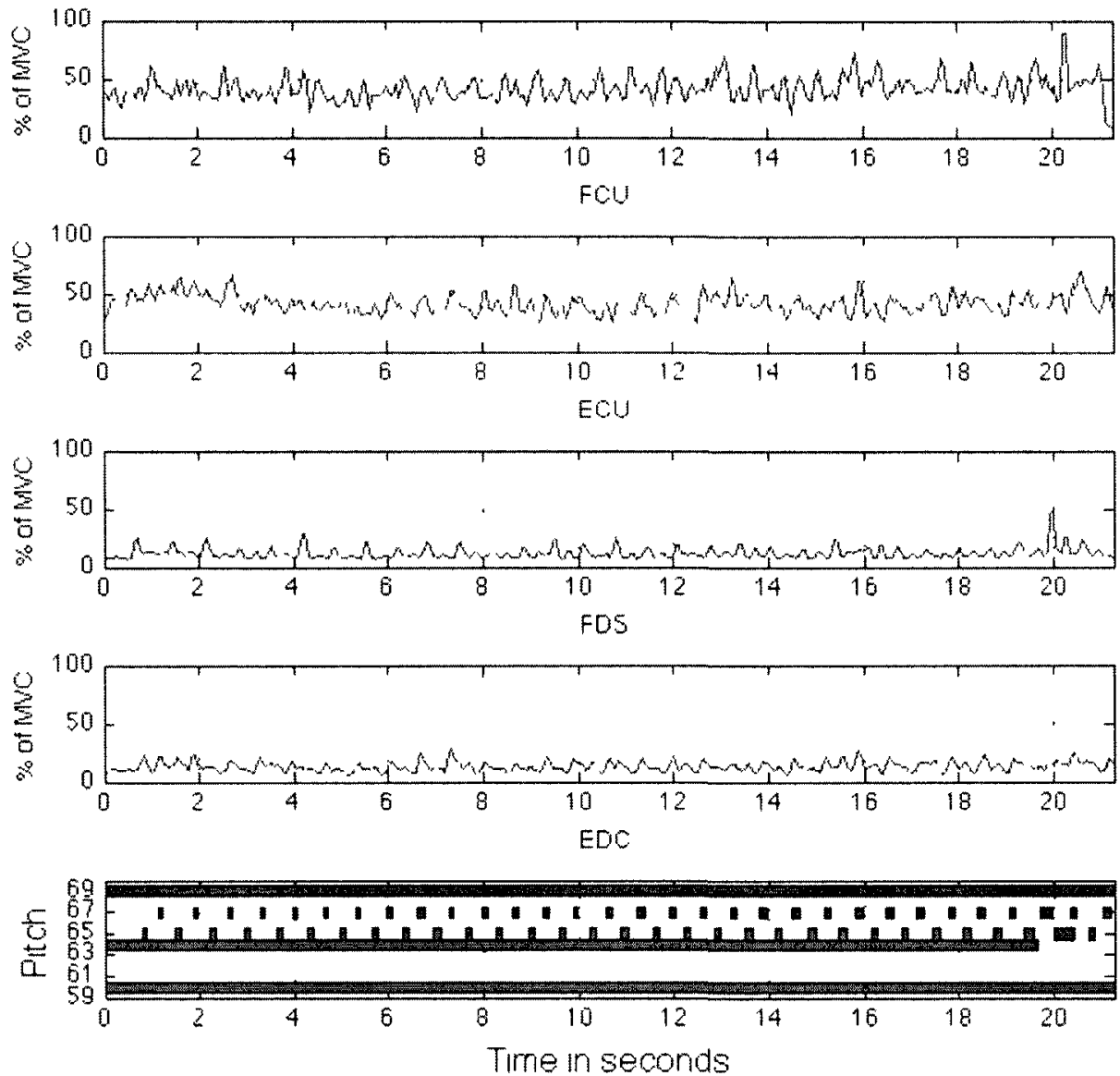

Figure K.18: Participant E010's EMG results: the upper plots show active muscle stiffness, expressed as a percentage of maximal voluntary contraction, while playing the second triad exercise (TR2) for four muscles of the right forearm: the FCU, the ECU, the FDS and the EDC. The corresponding MIDI data (note on, note off) are shown on the lower plot. 
Appendix L

Plots of Participants' Co-contraction Levels and MIDI Data While Playing Triad Exercises TR1 and TR2 

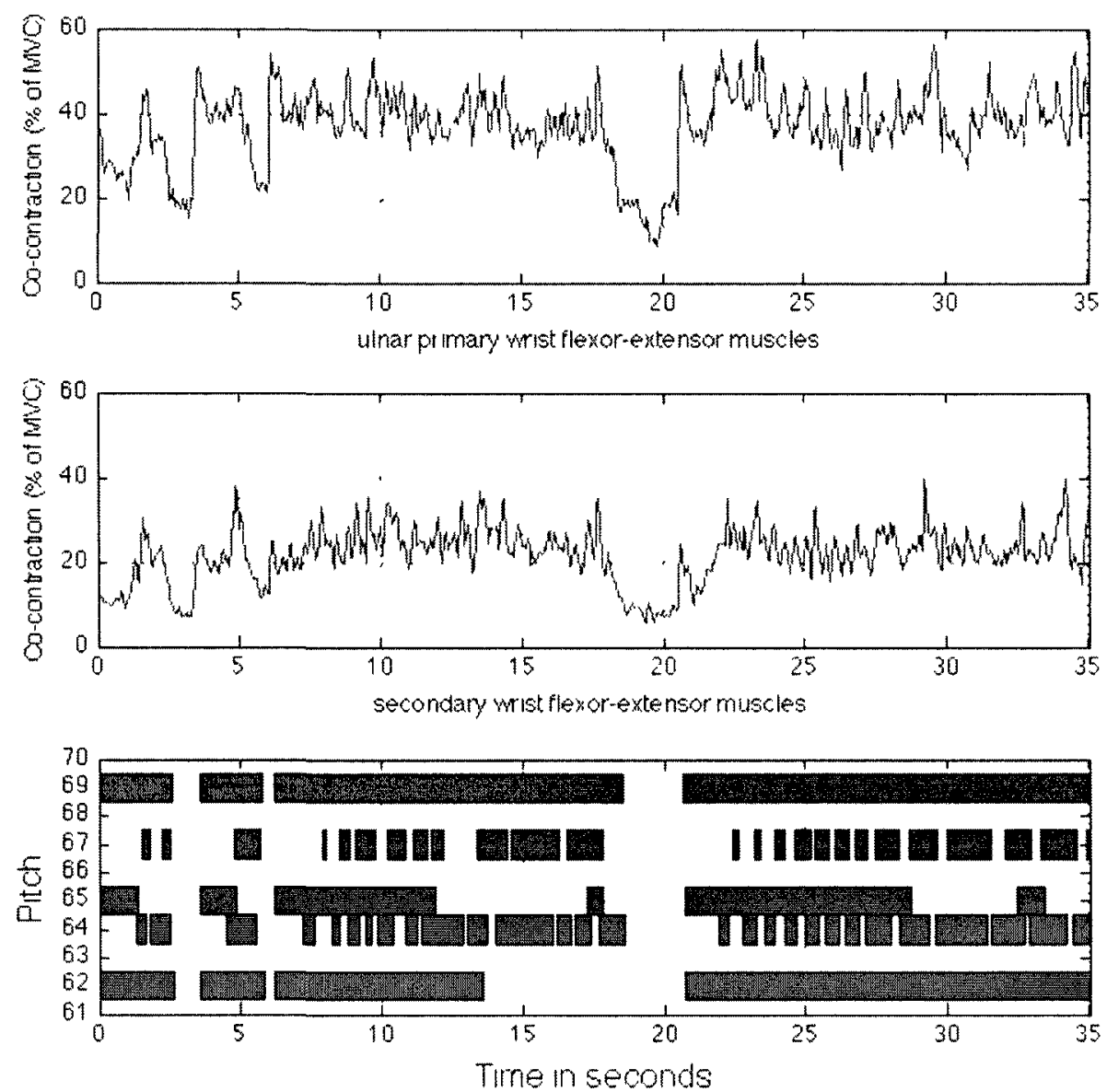

Figure L.1: Participant E002's EMG results: the upper plots show levels of muscular co-contraction, expressed as a percentage of maximal voluntary contraction, while playing the first triad exercise (TR1). The corresponding MIDI data (note on, note off) are shown on the lower plot. This participant lifted their fingers $>10 \%$ of the time, and thus their results are not valid for further analysis. 

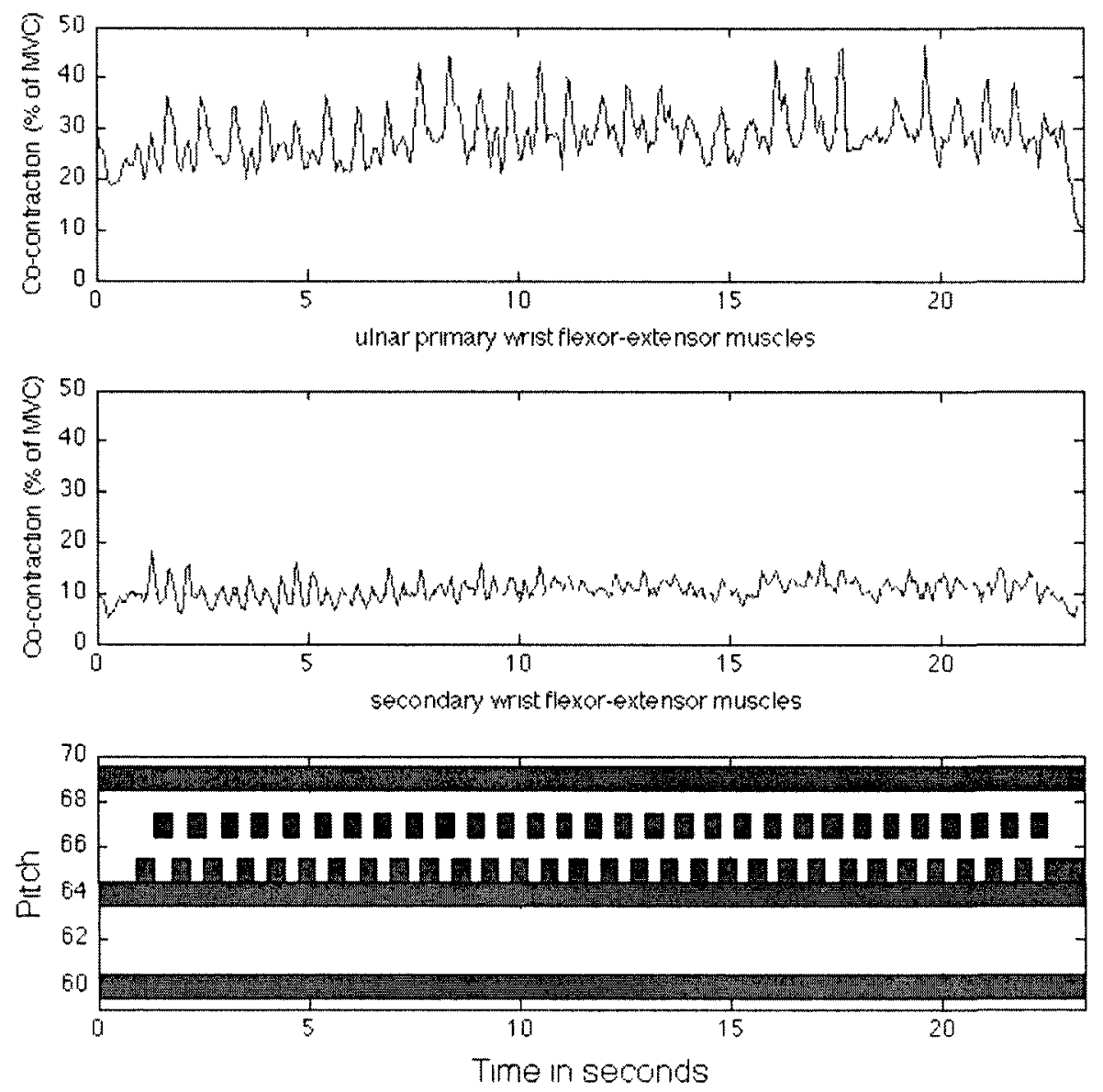

Figure L.2: Participant E002's EMG results: the upper plots show levels of muscular co-contraction, expressed as a percentage of maximal voluntary contraction, while playing the second triad exercise (TR2). The corresponding MIDI data (note on, note off) are shown on the lower plot. 

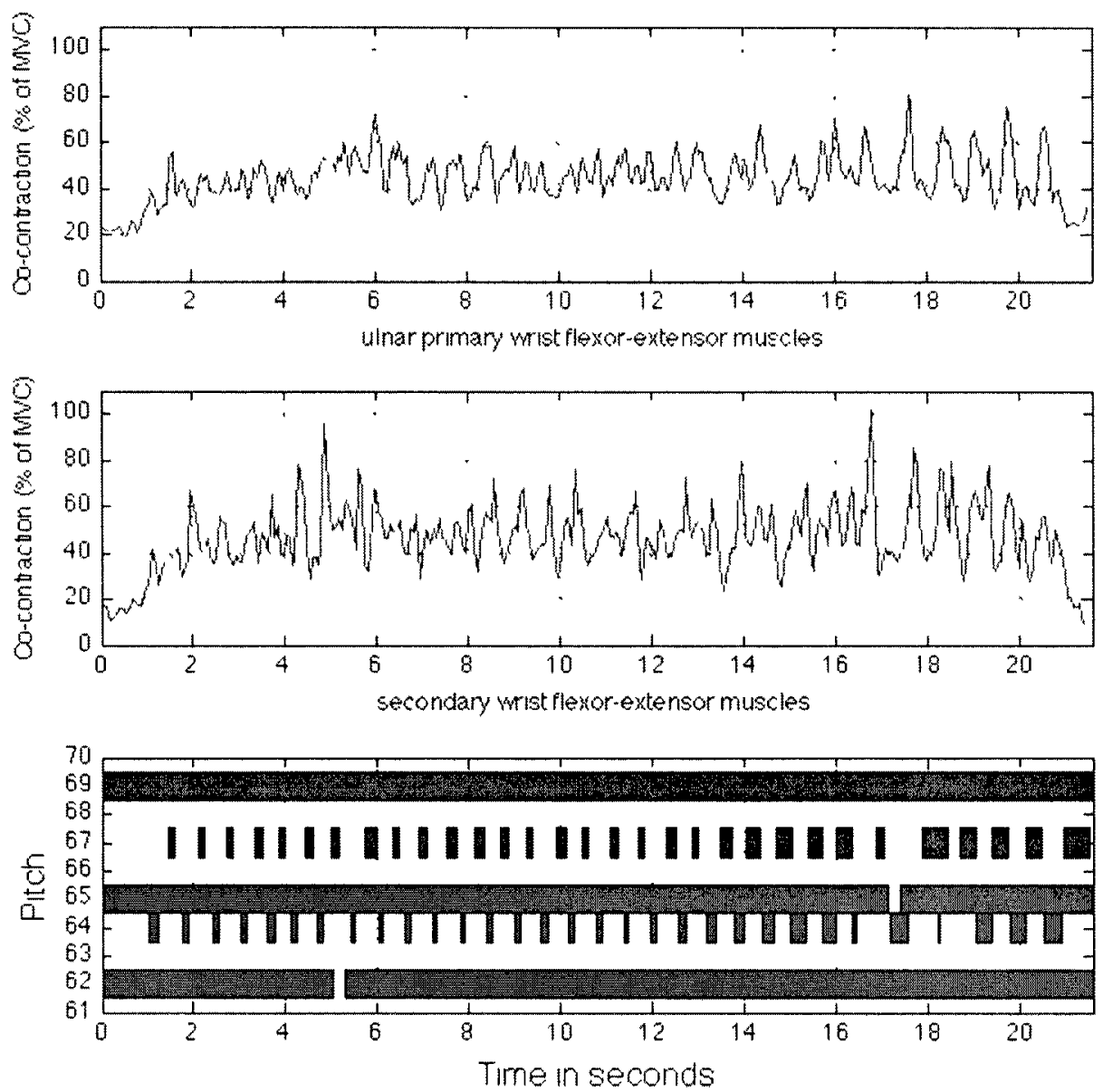

Figure L.3: Participant E003's EMG results: the upper plots show levels of muscular co-contraction, expressed as a percentage of maximal voluntary contraction, while playing the first triad exercise (TR1). The corresponding MIDI data (note on, note off) are shown on the lower plot. 

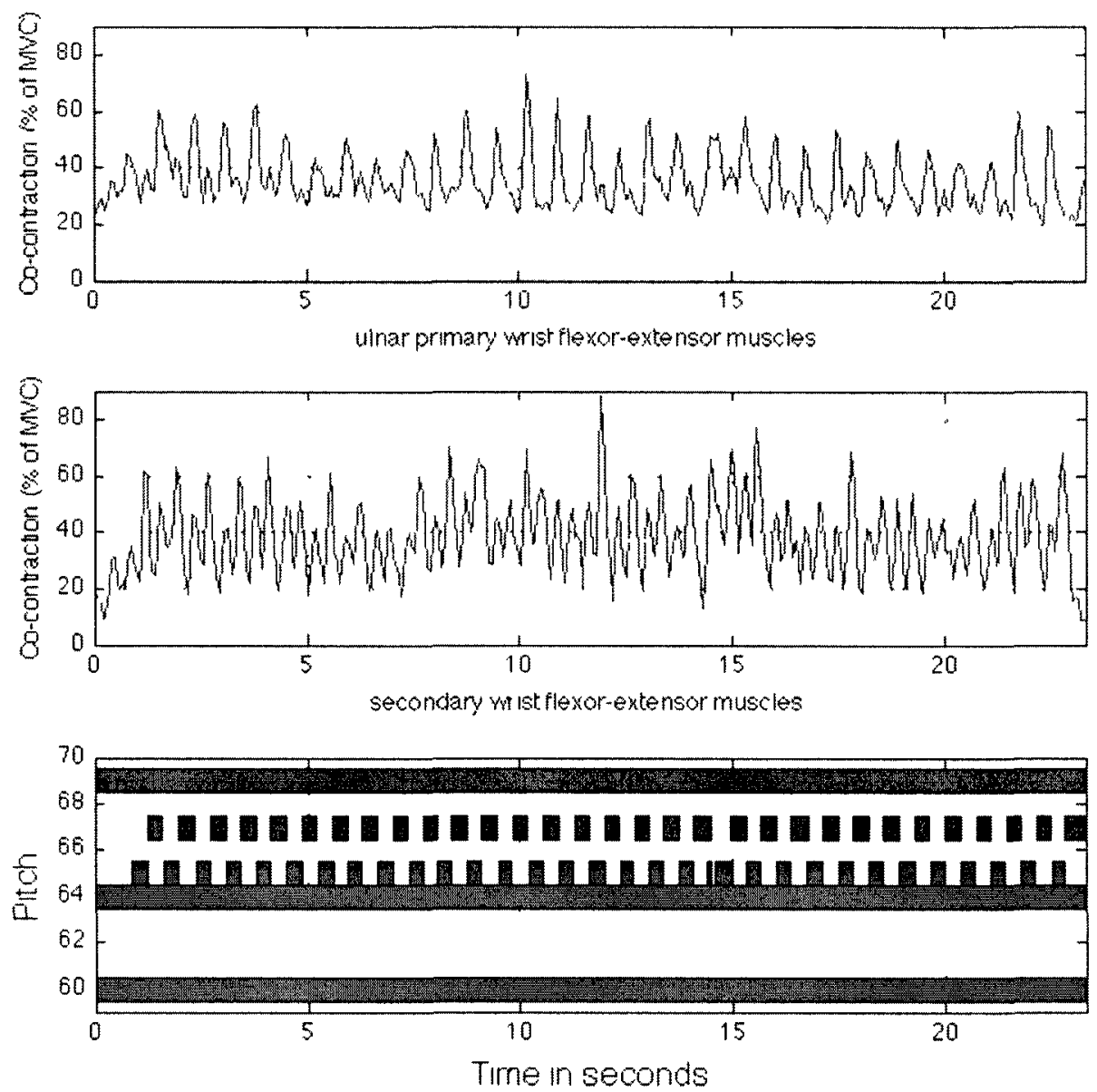

Figure L.4: Participant E003's EMG results: the upper plots show levels of muscular co-contraction, expressed as a percentage of maximal voluntary contraction, while playing the second triad exercise (TR2). The corresponding MIDI data (note on, note off) are shown on the lower plot. 

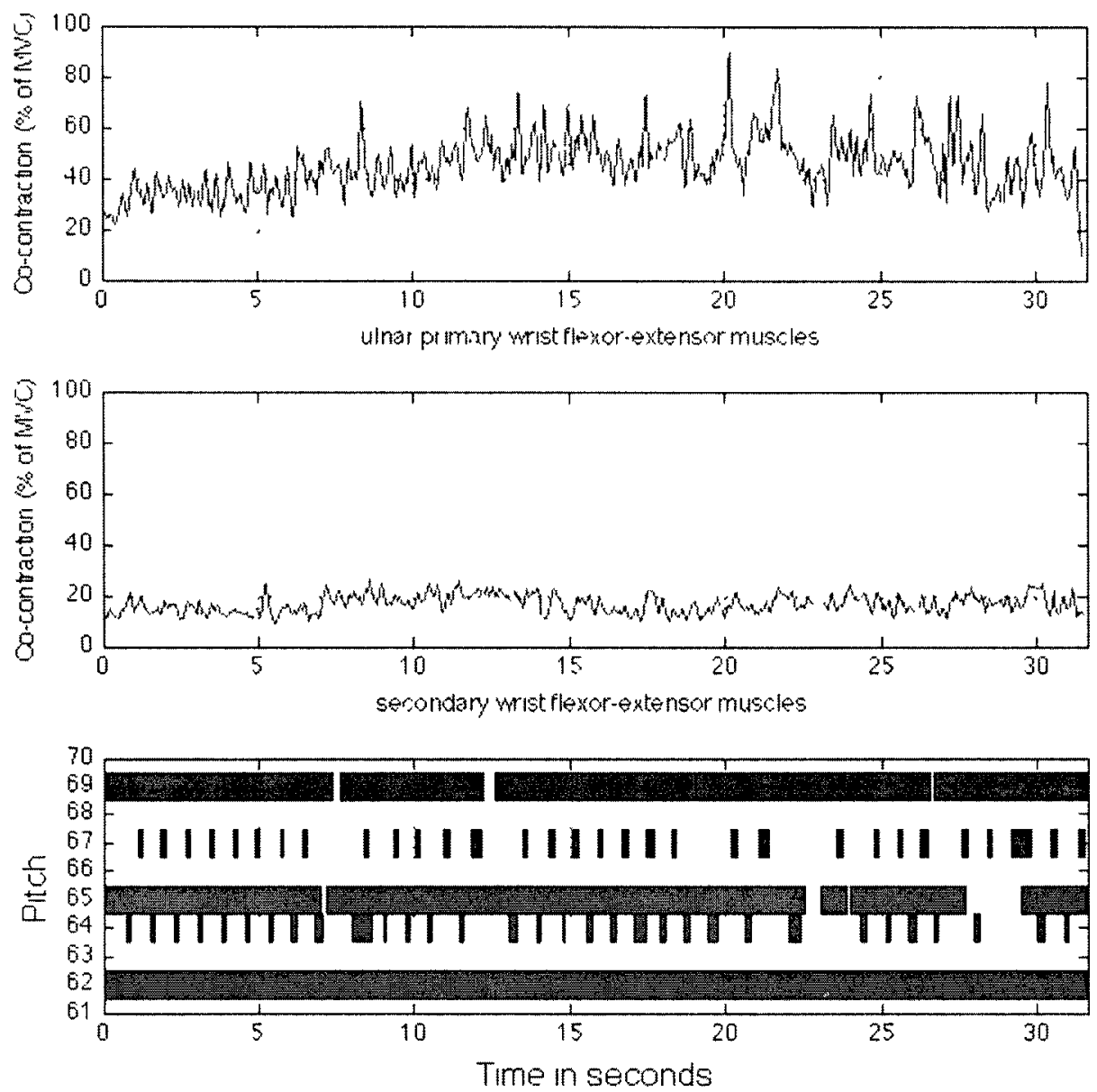

Figure L.5: Participant E004's EMG results: the upper plots show levels of muscular co-contraction, expressed as a percentage of maximal voluntary contraction, while playing the first triad exercise (TR1). The corresponding MIDI data (note on, note off) are shown on the lower plot. 

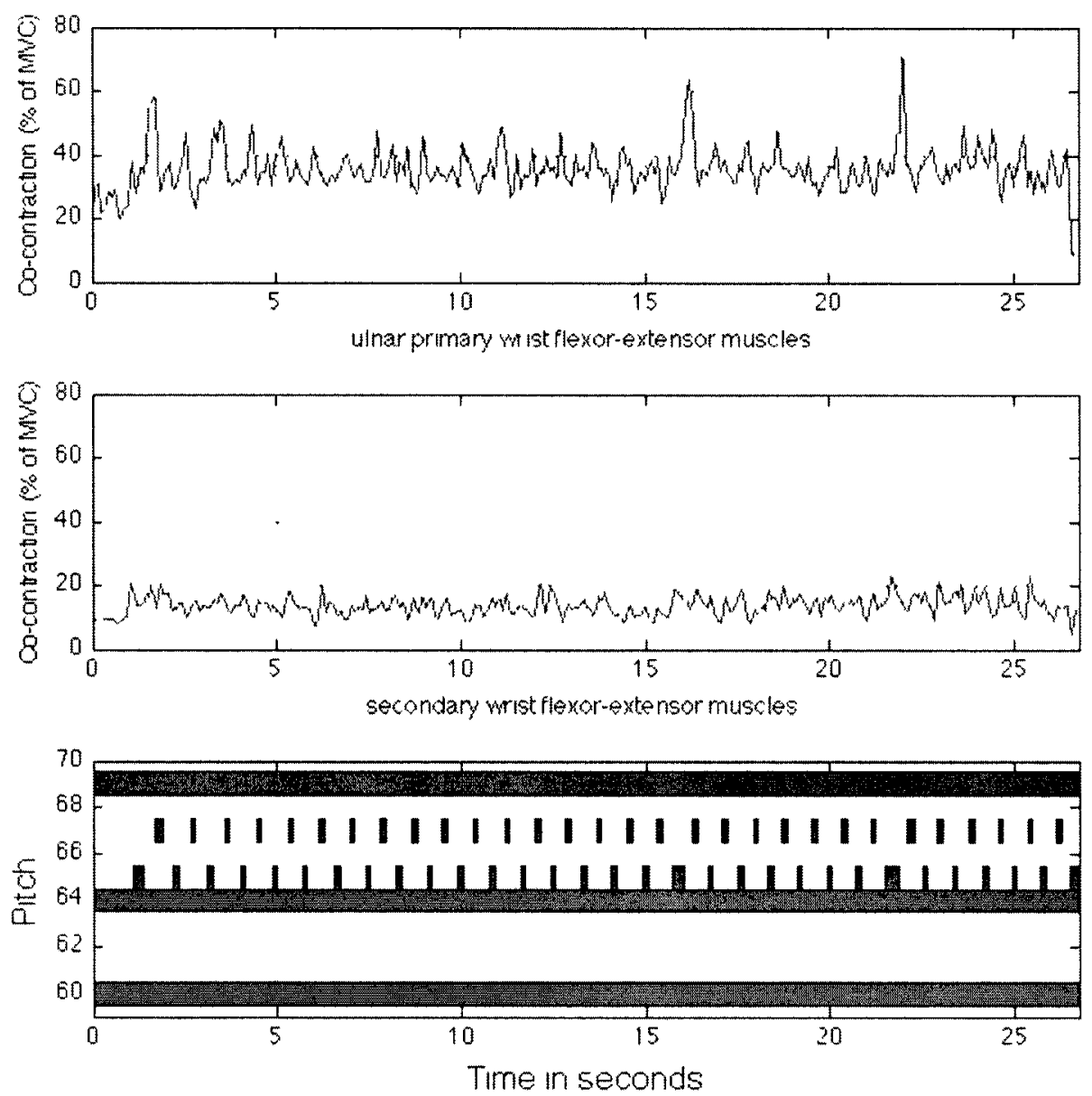

Figure L.6: Participant E004's EMG results: the upper plots show levels of muscular co-contraction, expressed as a percentage of maximal voluntary contraction, while playing the second triad exercise (TR2). The corresponding MIDI data (note on, note off) are shown on the lower plot. 

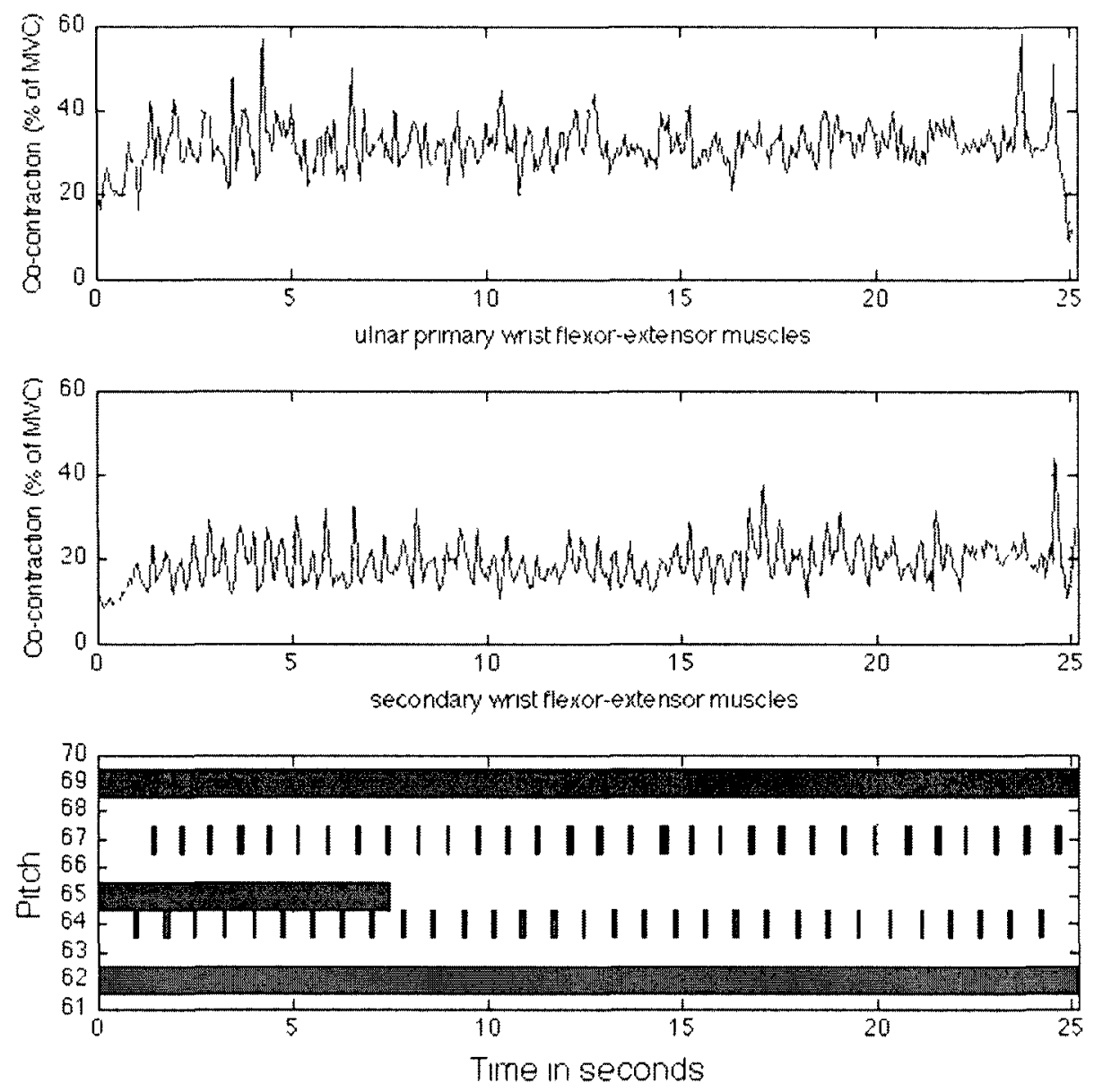

Figure L.7: Participant E005's EMG results: the upper plots show levels of muscular co-contraction, expressed as a percentage of maximal voluntary contraction, while playing the first triad exercise (TR1). The corresponding MIDI data (note on, note off) are shown on the lower plot. This participant lifted fr3 > $10 \%$ of the time, and thus their results are not valid for further analysis. 

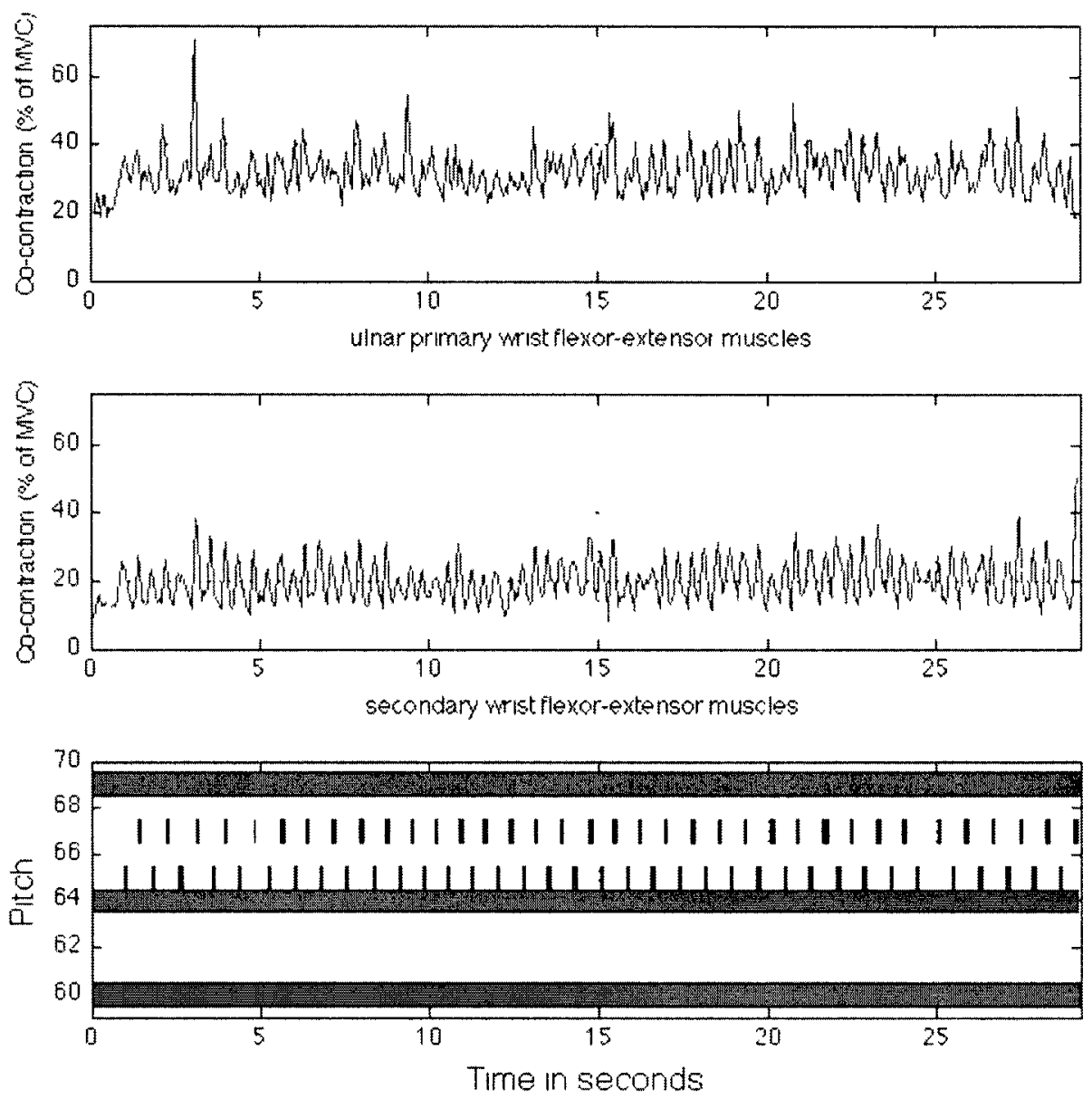

Figure L.8: Participant E005's EMG results: the upper plots show levels of muscular co-contraction, expressed as a percentage of maximal voluntary contraction, while playing the second triad exercise (TR2). The corresponding MIDI data (note on, note off) are shown on the lower plot. 

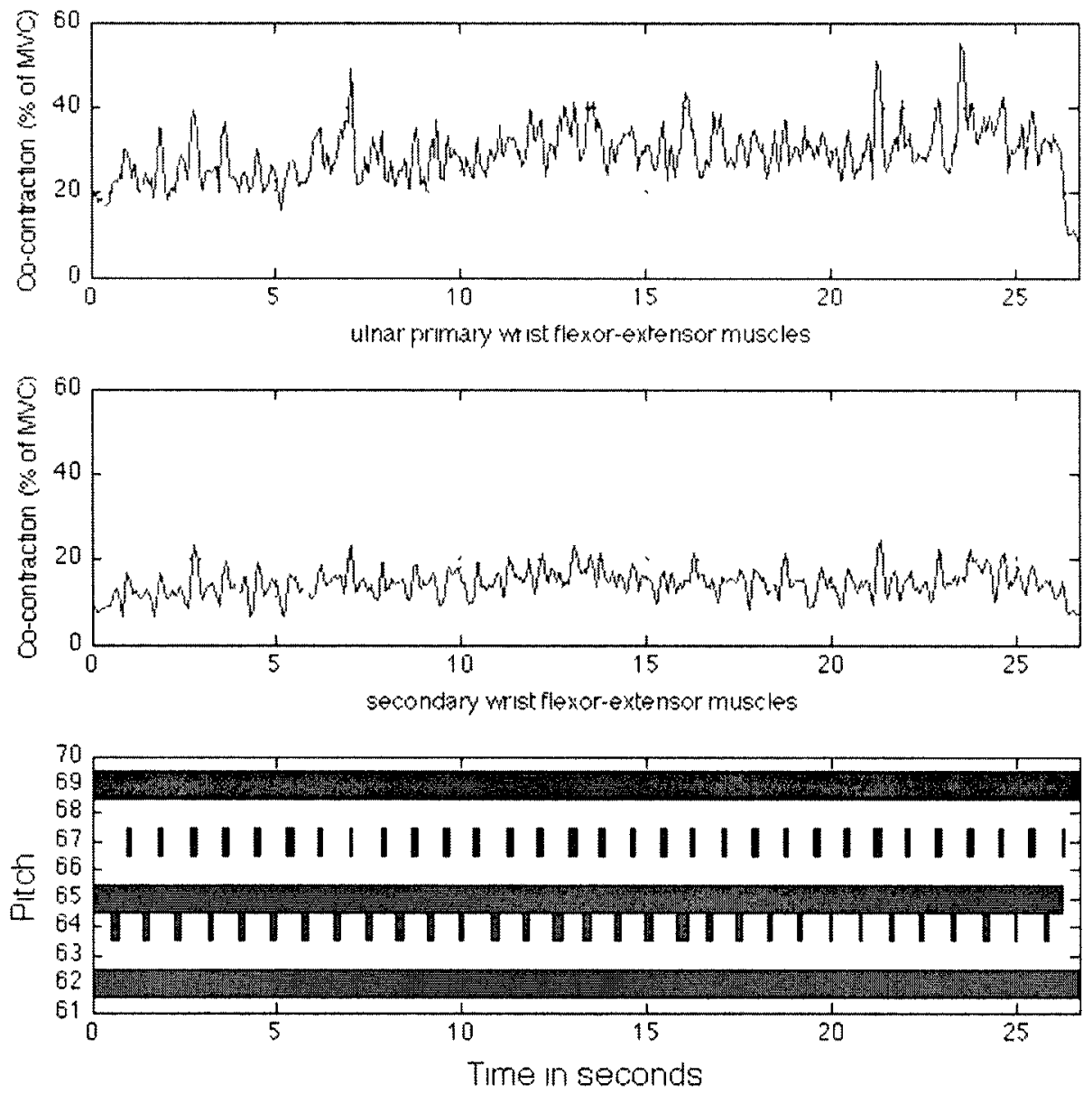

Figure L.9: Participant E006's EMG results: the upper plots show levels of muscular co-contraction, expressed as a percentage of maximal voluntary contraction, while playing the first triad exercise (TR1). The corresponding MIDI data (note on, note off) are shown on the lower plot. 

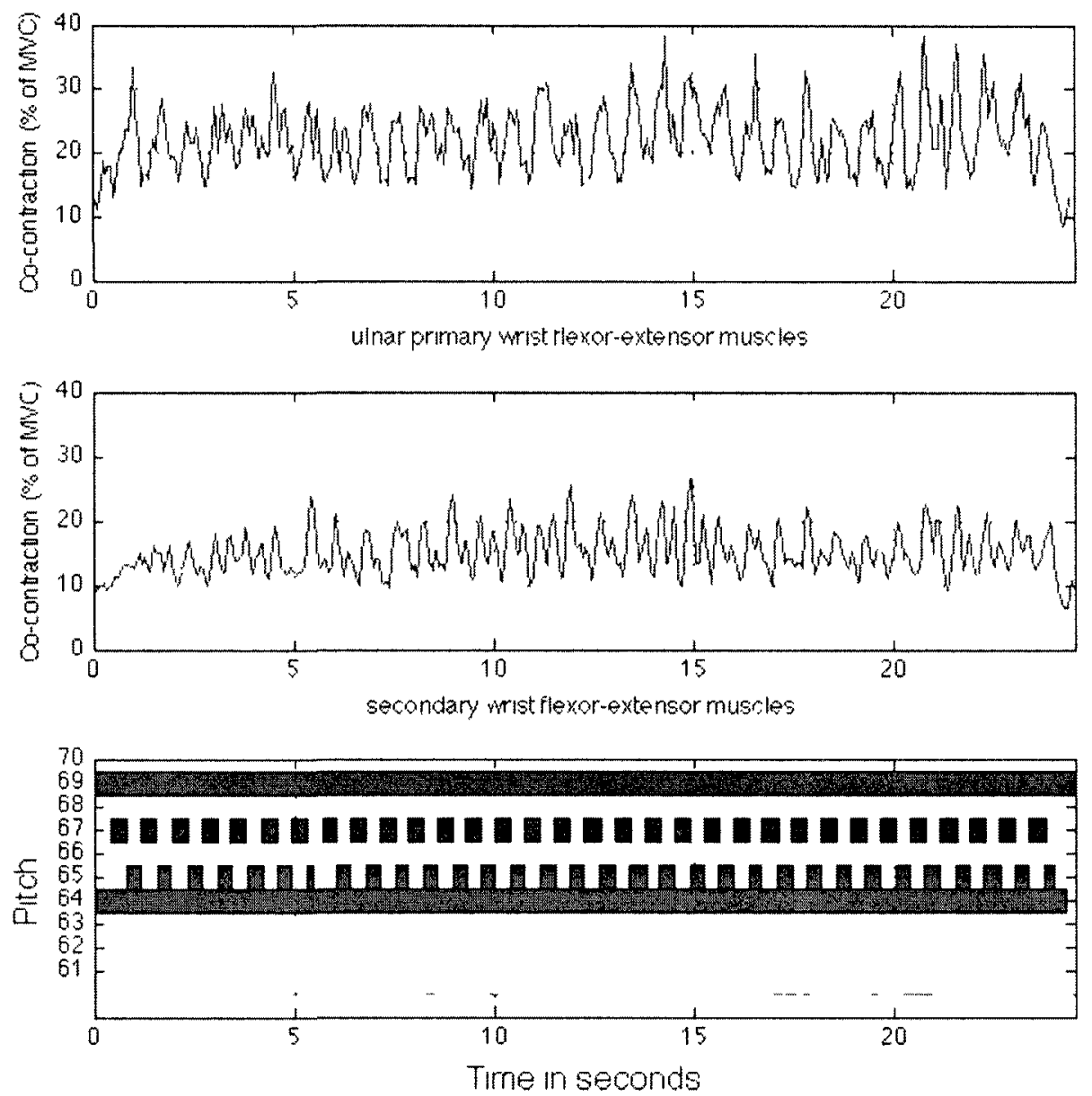

Figure L.10: Participant E006's EMG results: the upper plots show levels of muscular co-contraction, expressed as a percentage of maximal voluntary contraction, while playing the second triad exercise (TR2) The corresponding MIDI data (note on, note off) are shown on the lower plot. This participant lifted $\mathrm{fr} 1>$ $10 \%$ of the time, and thus their results are not valid for further analysis. 

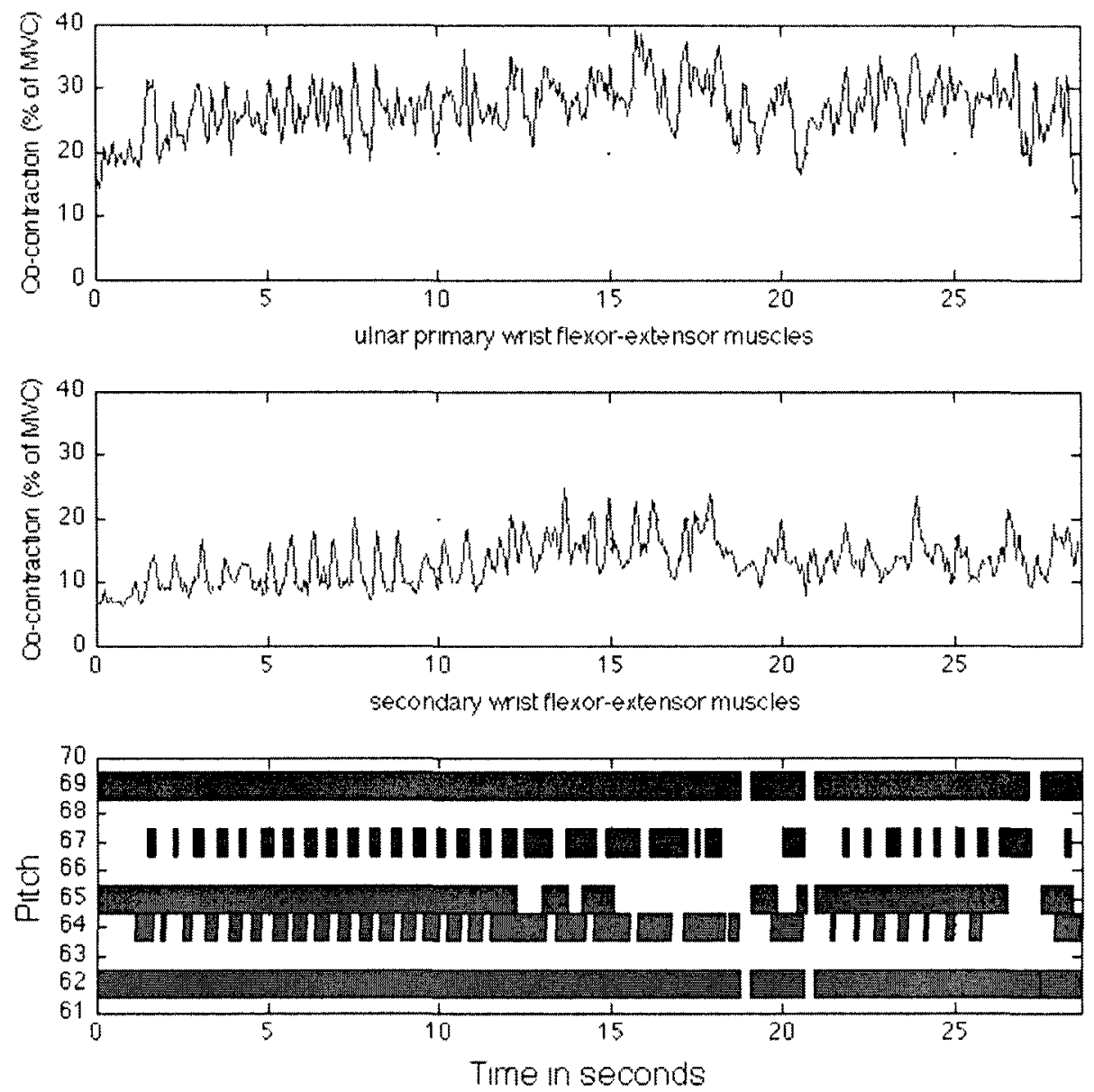

Figure L.11: Participant E007's EMG results: the upper plots show levels of muscular co-contraction, expressed as a percentage of maximal voluntary contraction, while playing the first triad exercise (TR1). The corresponding MIDI data (note on, note off) are shown on the lower plot. This participant lifted their fingers $>10 \%$ of the time, and thus their results are not valid for further analysis. 

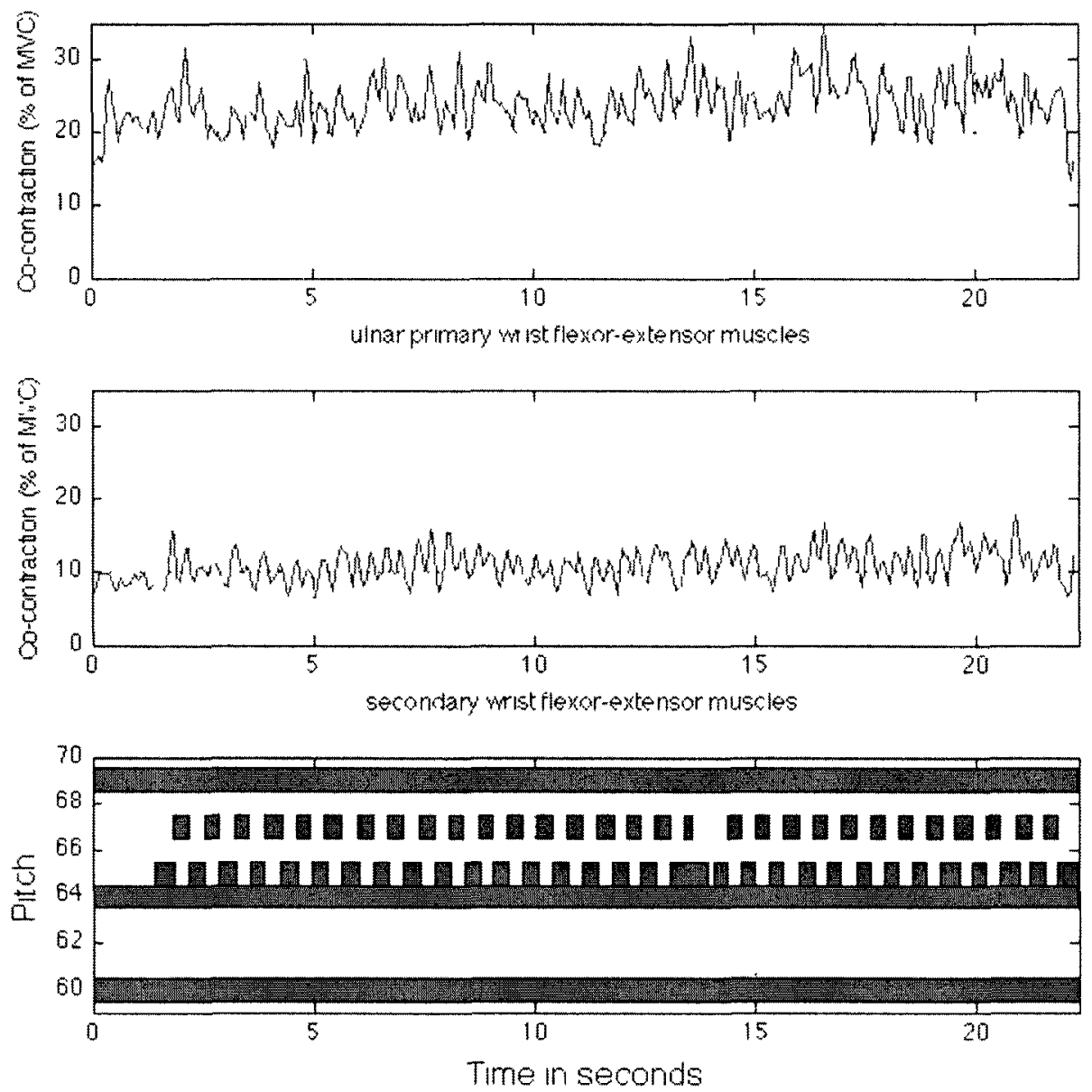

Figure L.12: Participant E007's EMG results: the upper plots show levels of muscular co-contraction, expressed as a percentage of maximal voluntary contraction, whlle playing the second triad exercise (TR2). The corresponding MIDI data (note on, note off) are shown on the lower plot. 

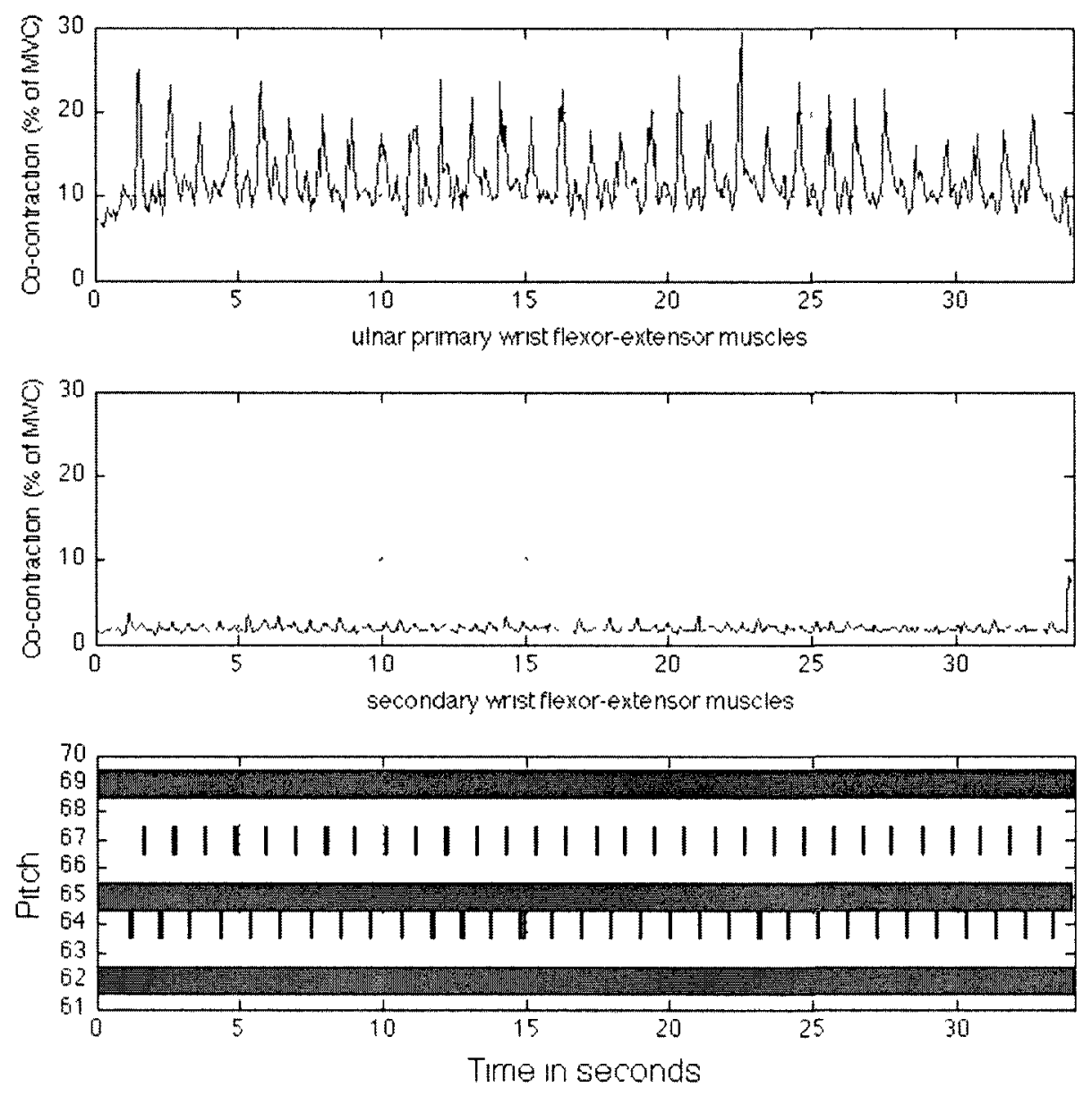

Figure L.13: Participant E008's EMG results: the upper plots show levels of muscular co-contraction, expressed as a percentage of maximal voluntary contraction, while playing the first triad exercise (TR1). The corresponding MIDI data (note on, note off) are shown on the lower plot. 

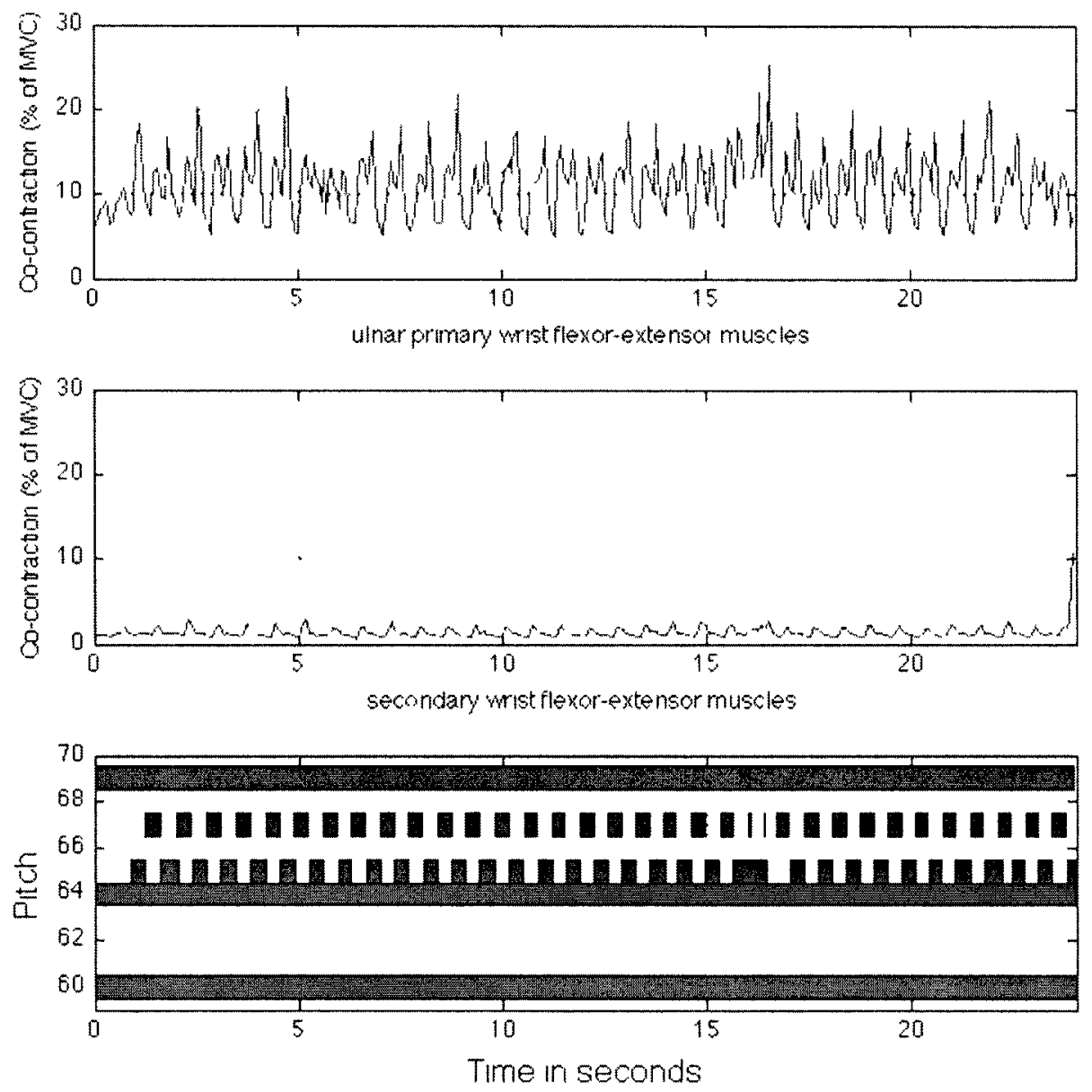

Figure L.14: Participant E008's EMG results: the upper plots show levels of muscular co-contraction, expressed as a percentage of maximal voluntary contraction, while playing the second triad exercise (TR2). The corresponding MIDI data. (note on, note off) are shown on the lower plot. 

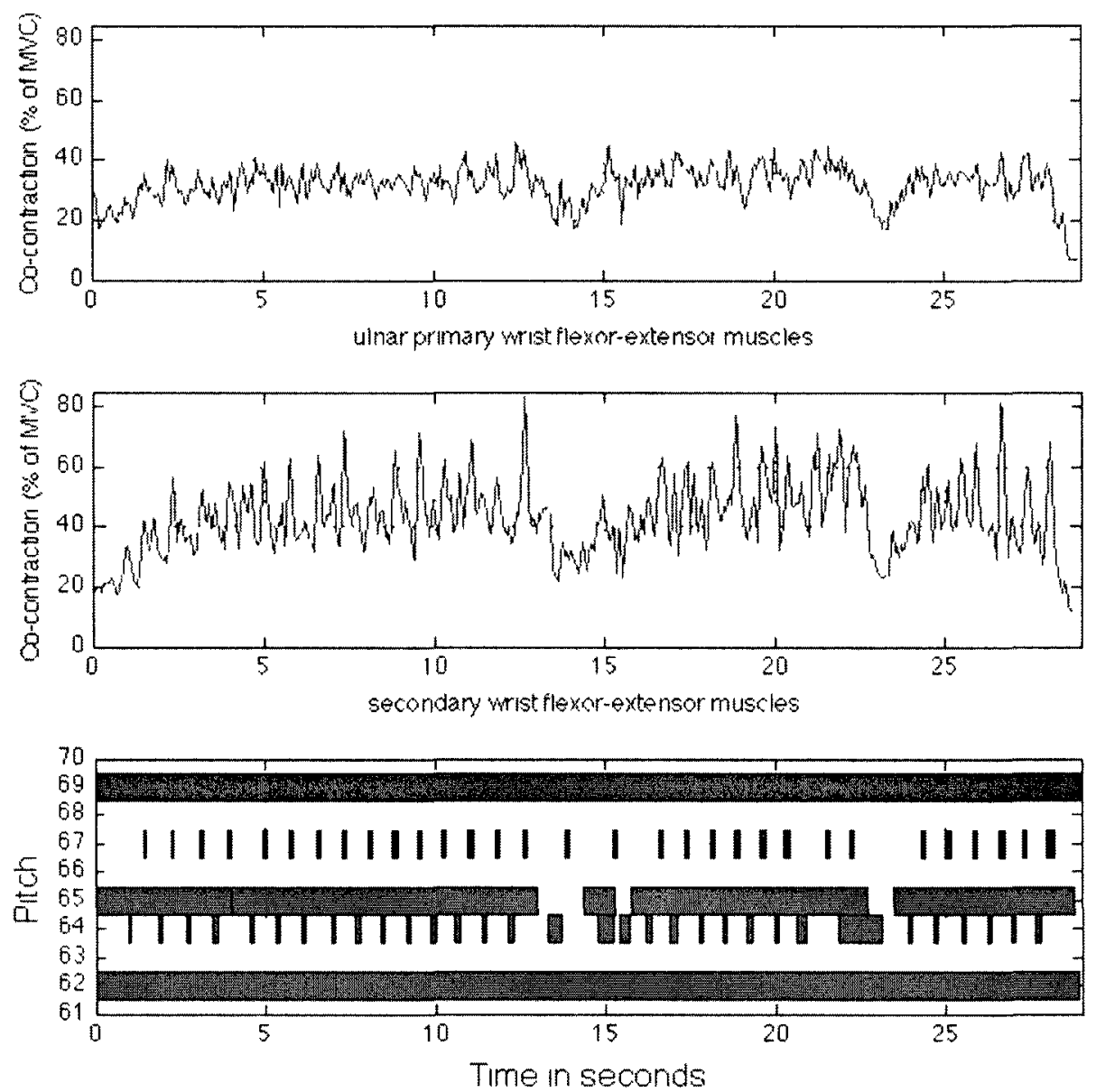

Figure L.15: Participant E009's EMG results: the upper plots show levels of muscular co-contraction, expressed as a percentage of maximal voluntary contraction, while playing the first triad exercise (TR1). The corresponding MIDI data (note on, note off) are shown on the lower plot. 

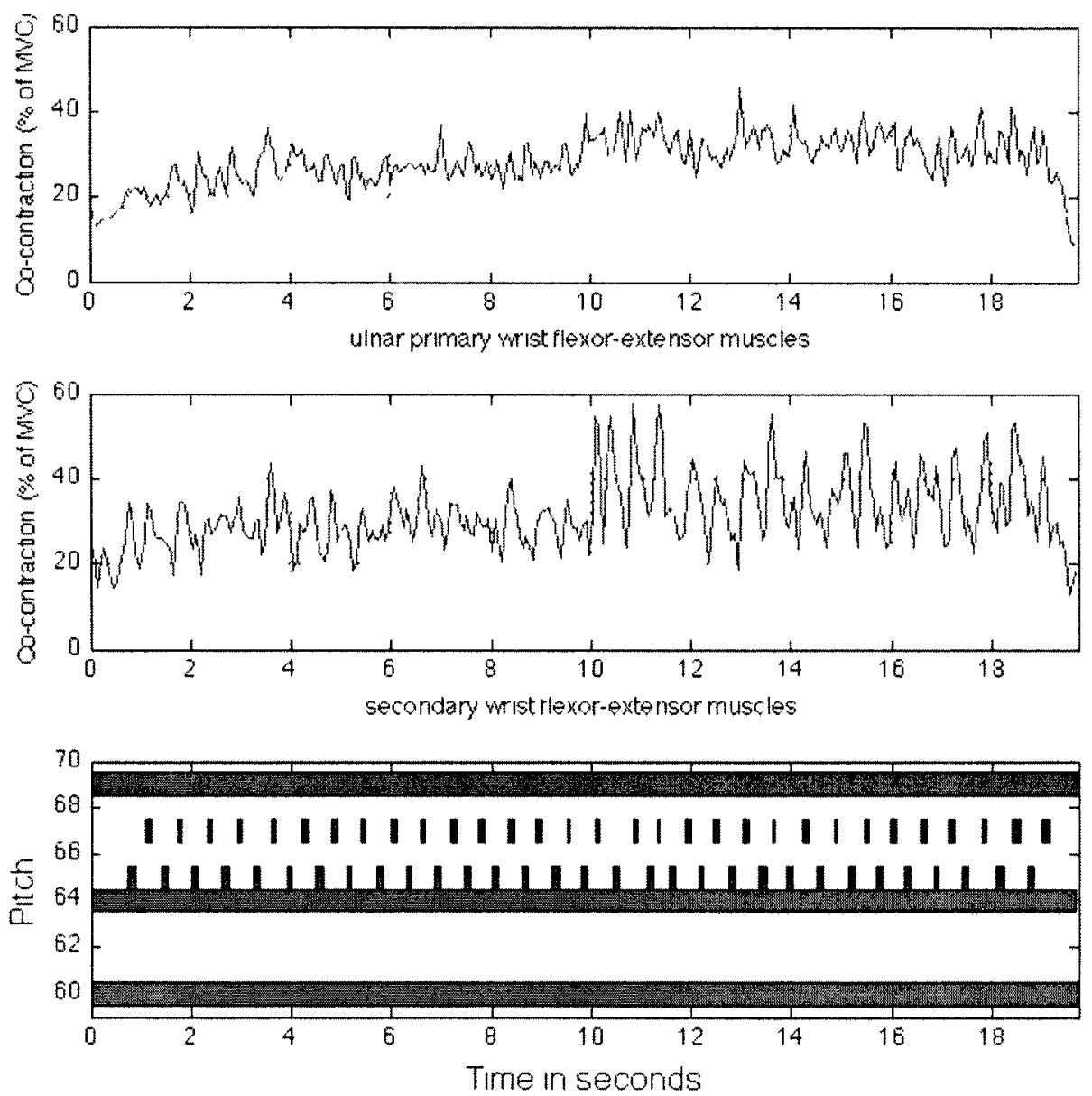

Figure L.16: Participant E009's EMG results: the upper plots show levels of muscular co-contraction, expressed as a percentage of maximal voluntary contraction, while playing the second triad exercise (TR2). The corresponding MIDI data (note on, note off) are shown on the lower plot. 

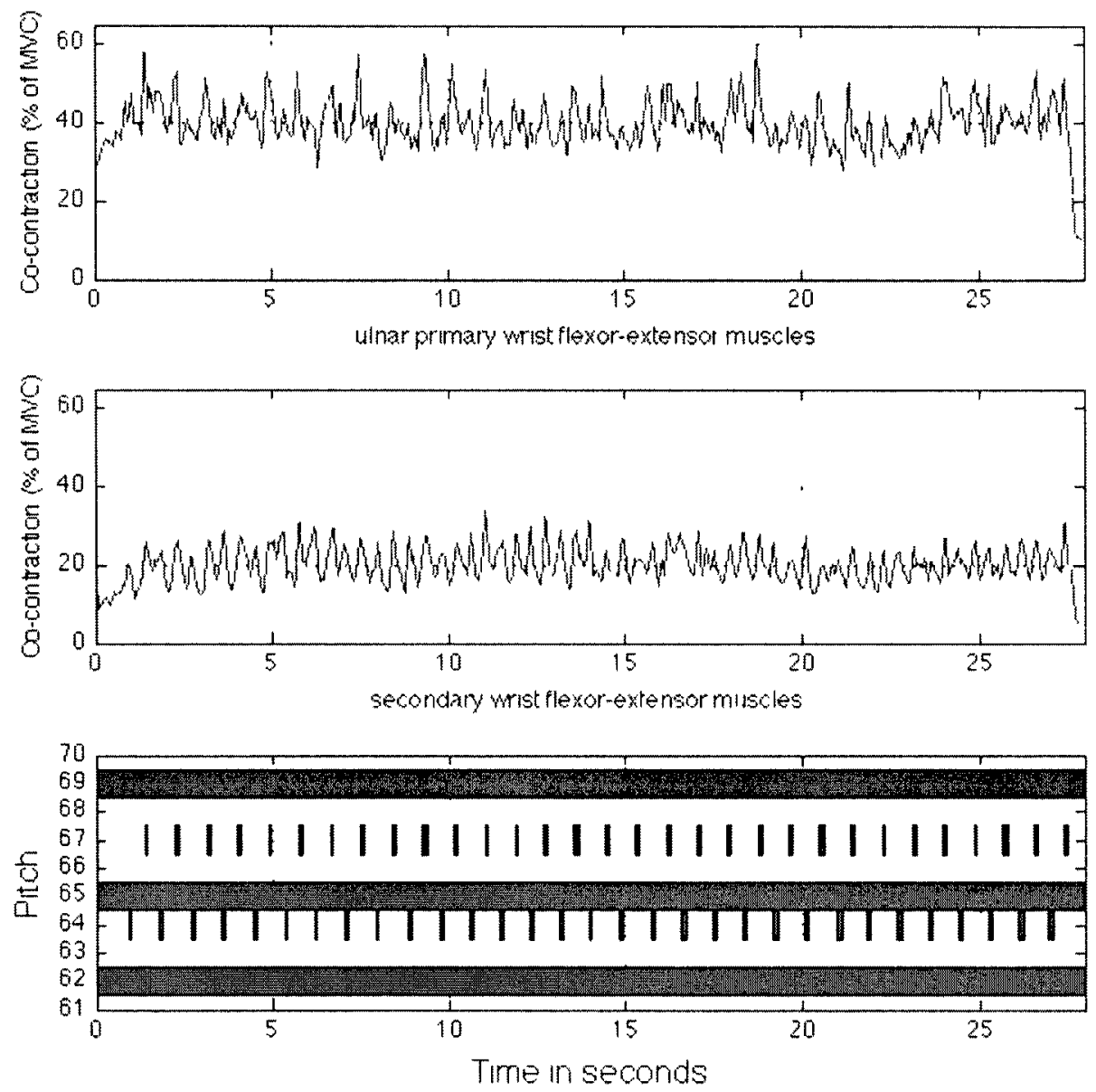

Figure L.17: Participant E010's EMG results: the upper plots show levels of muscular co-contraction, expressed as a percentage of maximal voluntary contraction, whlle playing the first triad exercise (TR1). The corresponding MIDI data (note on, note off) are shown on the lower plot. 

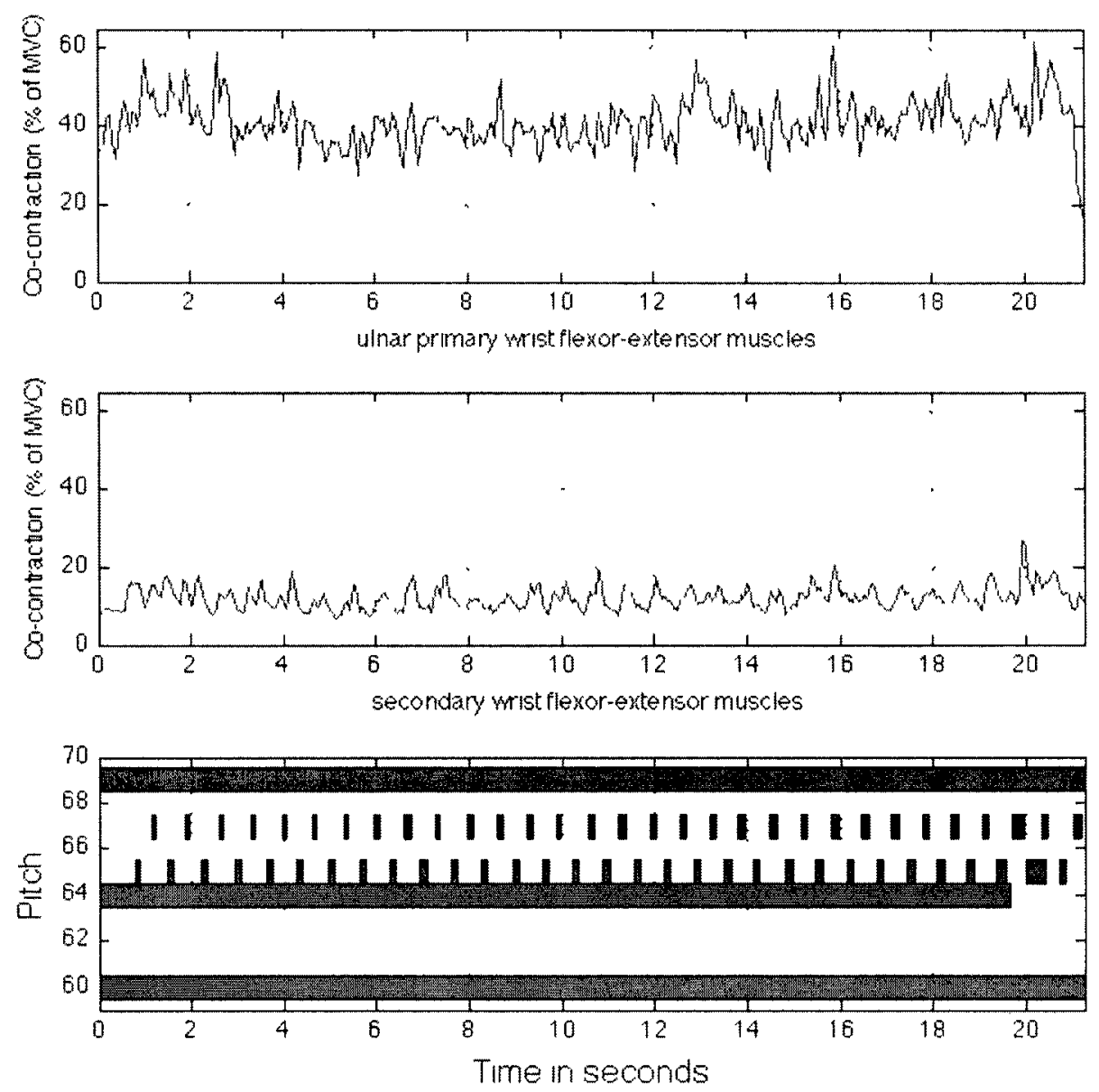

Figure L.18: Participant E010's EMG results: the upper plots show levels of muscular co-contraction, expressed as a percentage of maximal voluntary contraction, while playing the second triad exercise (TR2). The corresponding MIDI data (note on, note off) are shown on the lower plot. 
Appendix M

P-values from Student's t-test comparing the mTRs to mTRe of TR1 and TR2 
Table M.1: Resulting P-values from Student's t-tests comparing the mean start and end EMG values (comparing mTRs to mTRe) for both triad exercises

\begin{tabular}{|c|c|c|c|c|c|c|c|c|}
\hline & & & \multicolumn{6}{|c|}{ P-values } \\
\hline & & & $\mathrm{FCU}$ & ECU & FDS & EDC & $\mathrm{CC1}$ & $\mathrm{CC2}$ \\
\hline \multirow{18}{*}{ 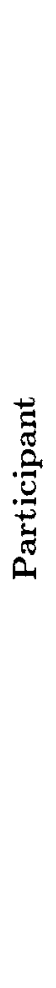 } & \multirow{9}{*}{ 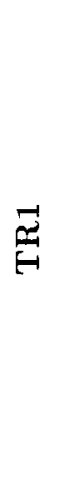 } & $\mathrm{E} 002$ & $122 \mathrm{E}-08$ & 0144 & $206 \mathrm{E}-05$ & $752 \mathrm{E}-03$ & $945 \mathrm{E}-03$ & $179 \mathrm{E}-04$ \\
\hline & & E003 & $798 \mathrm{E}-04$ & 0057 & 0464 & 0409 & $256 \mathrm{E}-03$ & 0101 \\
\hline & & E004 & 0016 & $641 \mathrm{E}-14$ & 0145 & $132 \mathrm{E}-12$ & $249 \mathrm{E}-06$ & $285 \mathrm{E}-09$ \\
\hline & & E005 & 0784 & 0094 & 0407 & $235 \mathrm{E}-03$ & 0190 & 0106 \\
\hline & & E006 & $587 \mathrm{E}-07$ & 0036 & $981 \mathrm{E}-03$ & $368 \mathrm{E}-03$ & $177 \mathrm{E}-11$ & $115 \mathrm{E}-03$ \\
\hline & & E007 & $770 \mathrm{E}-04$ & $250 \mathrm{E}-09$ & $331 \mathrm{E}-11$ & $101 \mathrm{E}-04$ & $124 \mathrm{E}-10$ & $116 \mathrm{E}-09$ \\
\hline & & E008 & 0293 & $256 \mathrm{E}-05$ & 0672 & 0082 & 0080 & 0285 \\
\hline & & E009 & 0089 & 0850 & 0014 & 0077 & 0014 & $802 \mathrm{E}-03$ \\
\hline & & E010 & 0348 & 0754 & 0953 & 0618 & 0619 & 0792 \\
\hline & \multirow{9}{*}{$\underset{\tilde{z}}{\tilde{H}}$} & E002 & $254 \mathrm{E}-07$ & 0017 & $979 \mathrm{E}-04$ & $358 \mathrm{E}-05$ & $331 \mathrm{E}-05$ & $267 \mathrm{E}-05$ \\
\hline & & E003 & 0356 & $770 \mathrm{E}-12$ & 0350 & 0284 & $300 \mathrm{E}-04$ & 0243 \\
\hline & & E004 & 0017 & 0600 & $545 \mathrm{E}-05$ & 0090 & 0044 & $194 \mathrm{E}-03$ \\
\hline & & E005 & 0902 & 0017 & 0591 & 0232 & 0360 & 0348 \\
\hline & & E006 & 0790 & 0341 & 0056 & $768 \mathrm{E}-03$ & 0131 & $987 \mathrm{E}-03$ \\
\hline & & E007 & $420 \mathrm{E}-03$ & $425 \mathrm{E}-03$ & 0129 & $600 \mathrm{E}-04$ & $194 \mathrm{E}-04$ & $899 \mathrm{E}-05$ \\
\hline & & E008 & 0804 & 0732 & 0155 & 0832 & 0897 & 0495 \\
\hline & & E009 & 0013 & $206 \mathrm{E}-04$ & $254 \mathrm{E}-04$ & $269 \mathrm{E}-03$ & $357 \mathrm{E}-11$ & $207 \mathrm{E}-05$ \\
\hline & & E010 & 4 71E-03 & 0064 & 0960 & 0112 & 0259 & 0241 \\
\hline
\end{tabular}


Appendix $\mathbf{N}$

Average Key Velocity in Sections of Ann Southam Piece 


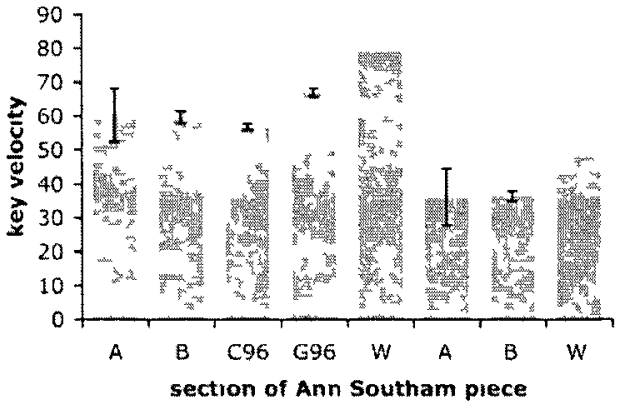

(a) Attempt 1

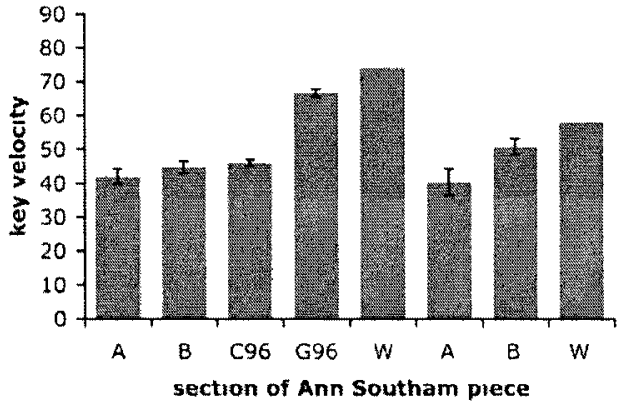

(b) Attempt 2

Figure N.1: Mean key velocity of sections A, B, C96, G96, W, A', B' and W' in Ann Southam piece for particıpant E002 Mean key velocities are shown for (a) a.t.tempt 1 - blue, and (b) attempt 2 - red Error bars represent 95\% confidence intervals There are no error bars for $W$ and $W$ ' because these sections are each made up of only one note 


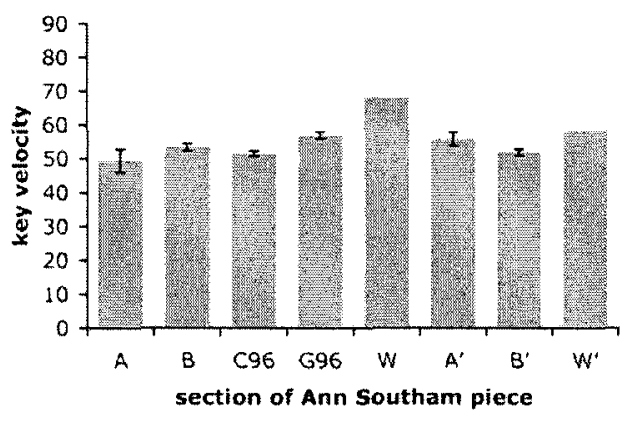

(a) Attempt 1

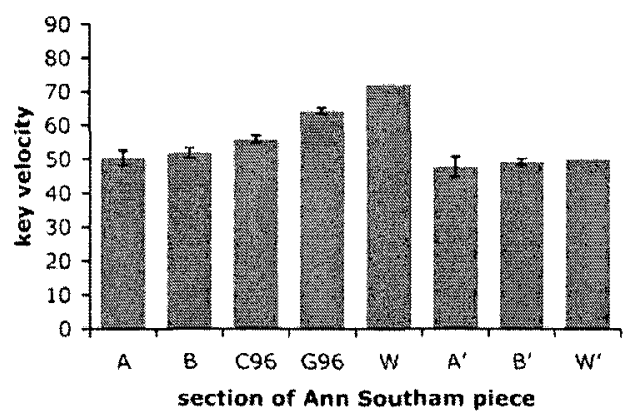

(b) Attempt 2

Figure N.2: Mean key velocity of sections A, B, C96, G96, W, A', B' and W' in Ann Southam piece for participant E003. Mean key velocities are shown for (a) attempt 1 - blue, and (b) attempt 2 - red. Error bars represent $95 \%$ confidence intervals. There are no error bars for $W$ and $W$ ' because these sections are each made up of only one note.

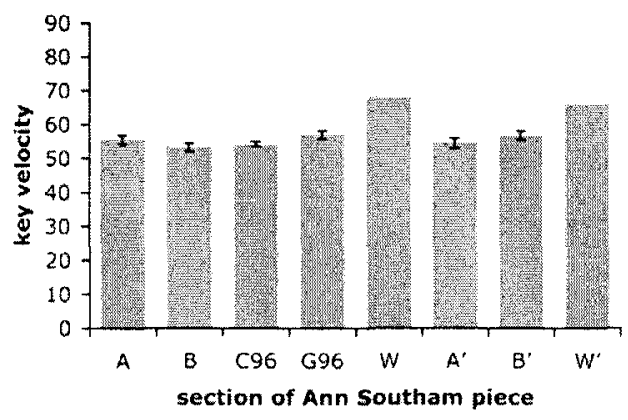

(a) Attempt 1

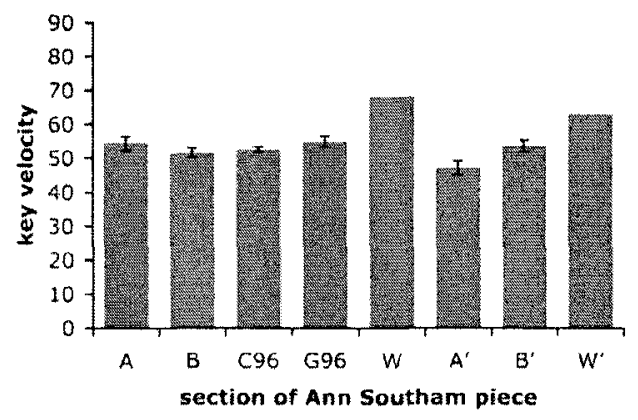

(b) Attempt 2

Figure N.3: Mean key velocity of sections A, B, C96, G96, W, A', B' and W' in Ann Southam piece for participant E004. Mean key velocities are shown for (a) attempt 1 - blue, and (b) attempt 2 - red.Error bars represent $95 \%$ confidence intervals. There are no error bars for $W$ and $W$ ' because these sections are each made up of only one note. 


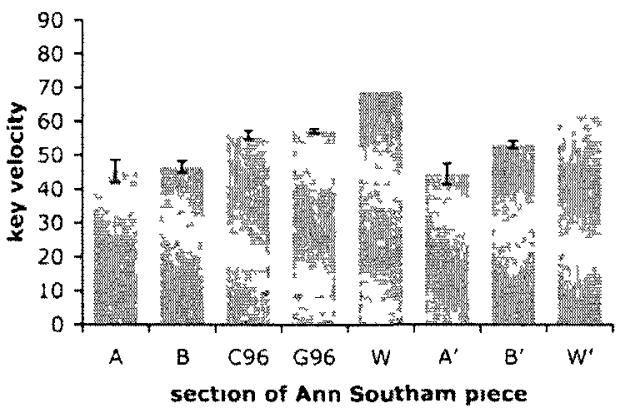

(a) Attempt 1

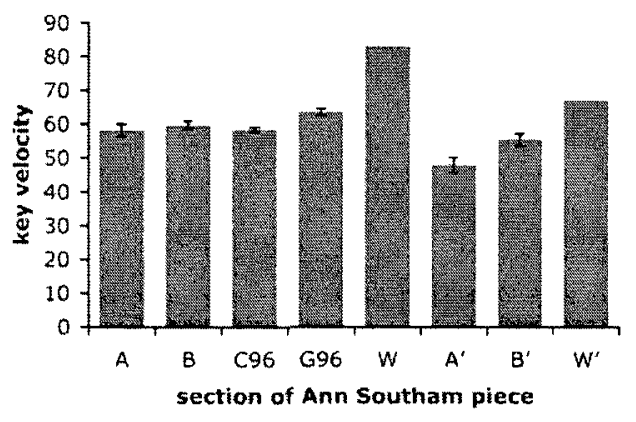

(b) Attempt 2

Figure N.4: Mean key velocity of sections A, B, C96, G96, W, A', B' and W' in Ann Southam piece for participant E005. Mean key velocities are shown for (a) attempt 1 - blue, and (b) attempt 2 - red. Error bars represent $95 \%$ confidence intervals. There are no error bars for $\mathrm{W}$ and $\mathrm{W}$ ' because these sections are each made up of only one note.

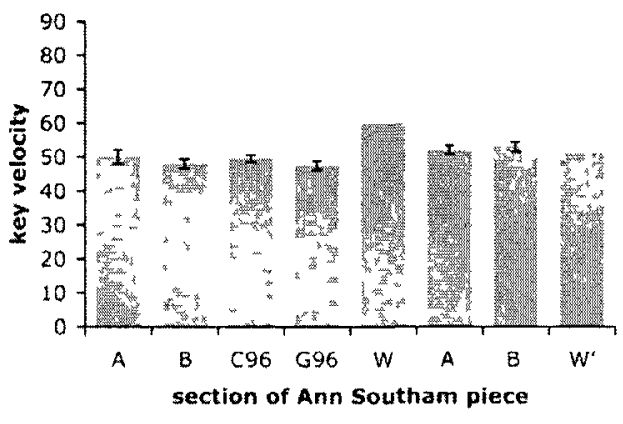

(a) Attempt 1

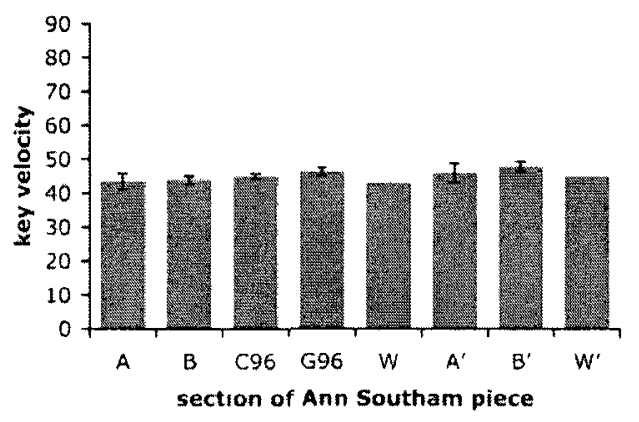

(b) Attempt 2

Figure N.5: Mean key velocity of sections A, B, C96, G96, W, A', B' and W' in Ann Southam piece for participant E006. Mean key velocities are shown for (a) attempt 1 - blue, and (b) attempt 2 - red. Error bars represent $95 \%$ confidence intervals. There are no error bars for $W$ and $W$ ' because these sections are each made up of only one note. 


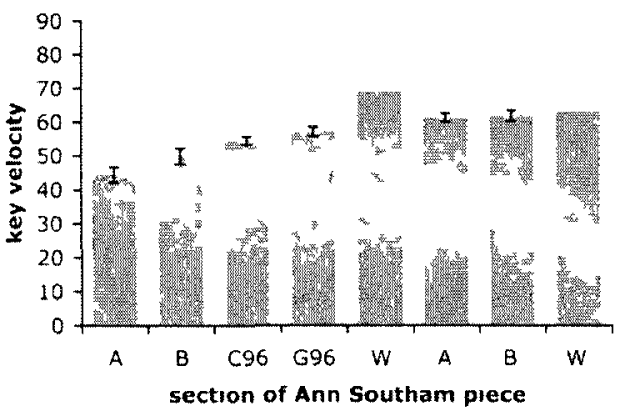

(a) Attempt 1

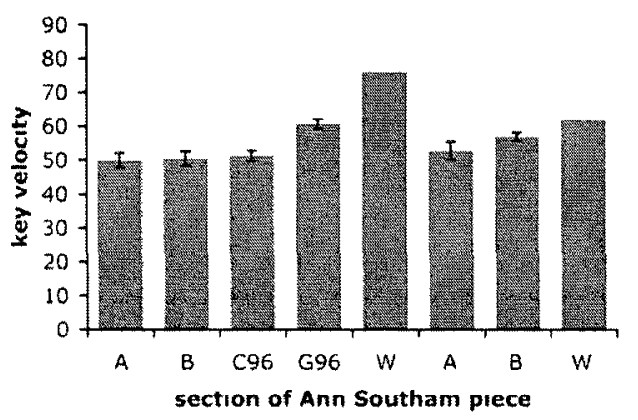

(b) Attempt 2

Figure N.6: Mean key velocity of sections A, B, C96, G96, W, A', B' and W' in Ann Southam piece for participant E007 Mean key velocities are shown for (a) attempt 1 - blue, and (b) attempt 2 - red Error bars represent 95\% confidence intervals There are no error bars for $W$ and $W$ ' because these sections are each made up of only one note

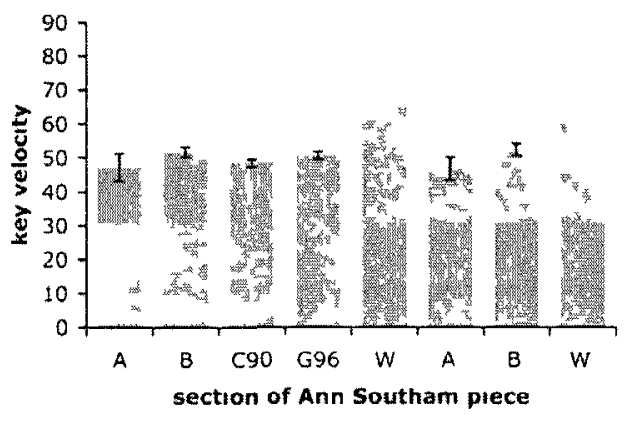

(a) Attempt 1

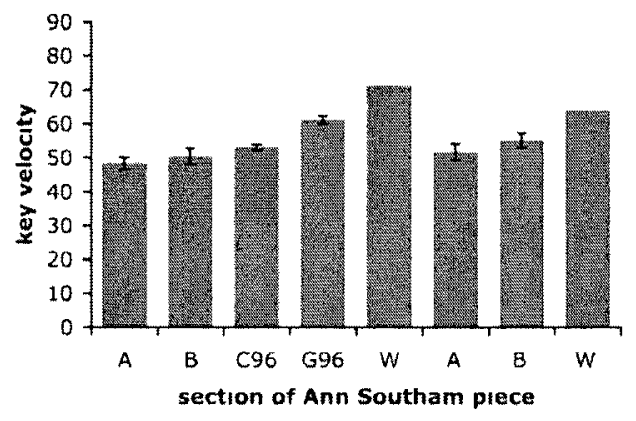

(b) Attempt 2

Figure N.7: Mean key velocity of sections A, B, C90 (attempt 1), C96 (attempt 2), G96, W, A', B' and W' in Ann Southam piece for participant E008 Mean key velocities are shown for (a) attempt 1 - bluc, and (b) attempt 2 - red Error bars represent $95 \%$ confidence intervals There are no error bars for $W$ and $W$ ' because these sections are each made up of only one note 


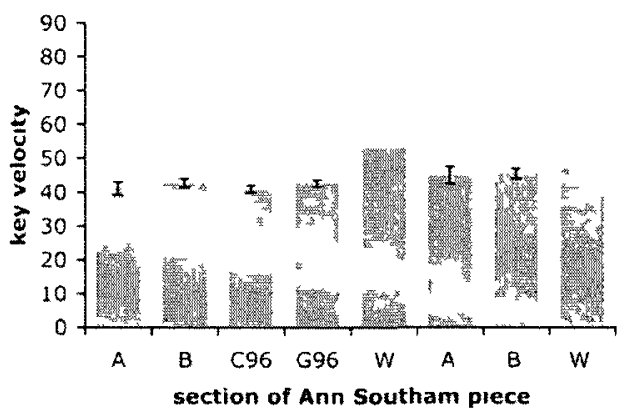

(a) Attempt 1

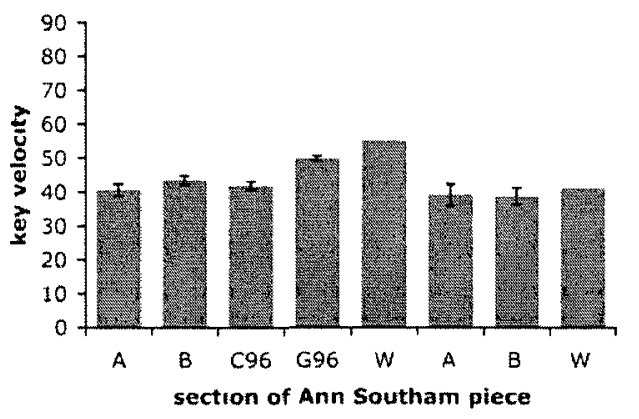

(b) Attempt 2

Figure N.8: Mean key velocity of sections A, B, C96, G96, W, A', B' and W' in Ann Southam piece for participant E009 Mean key velocities are shown for (a) attempt 1 - blue, and (b) attempt 2 - red Error bars represent $95 \%$ confidence intervals There are no error bars for $\mathrm{W}$ and $\mathrm{W}$ ' because these sections are each made up of only one note

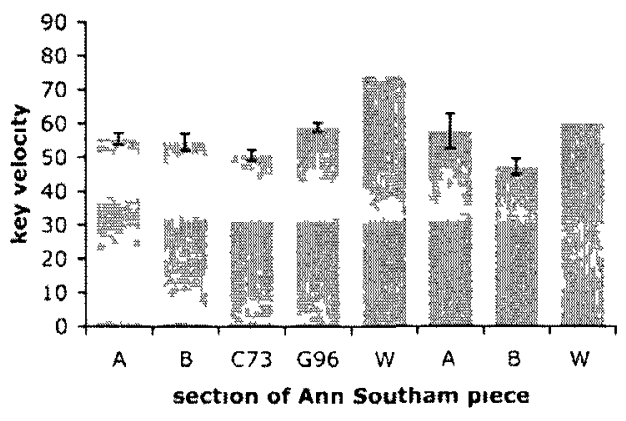

(a) Attempt 1

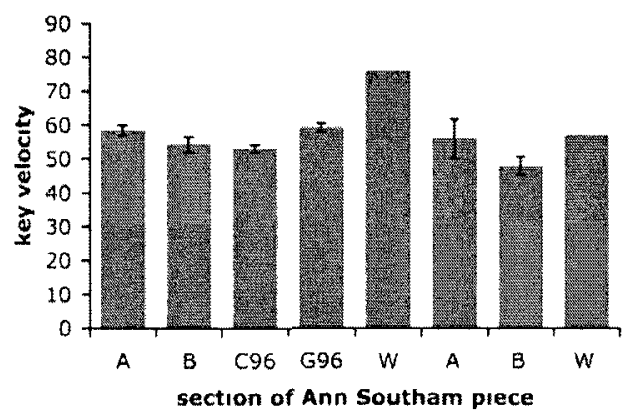

(b) Attempt 2

Figure N.9: Mean key velocity of sections A, B, C73 (attempt 1), C96 (attempt 2), G96, W, A', B' and W' in Ann Southam piece for participant E010 Mean key velocities are shown for (a) attempt 1 - blue, and (b) attempt 2 - red Error bars represent $95 \%$ confidence intervals There are no error bars for $\mathrm{W}$ and $\mathrm{W}$ ' because these sections are each made up of only one note 
Appendix $\mathrm{O}$

Plots of Participants' Active Muscle

Stiffnesses and MIDI Data While Playing

the Piece Composed by Ann Southam 

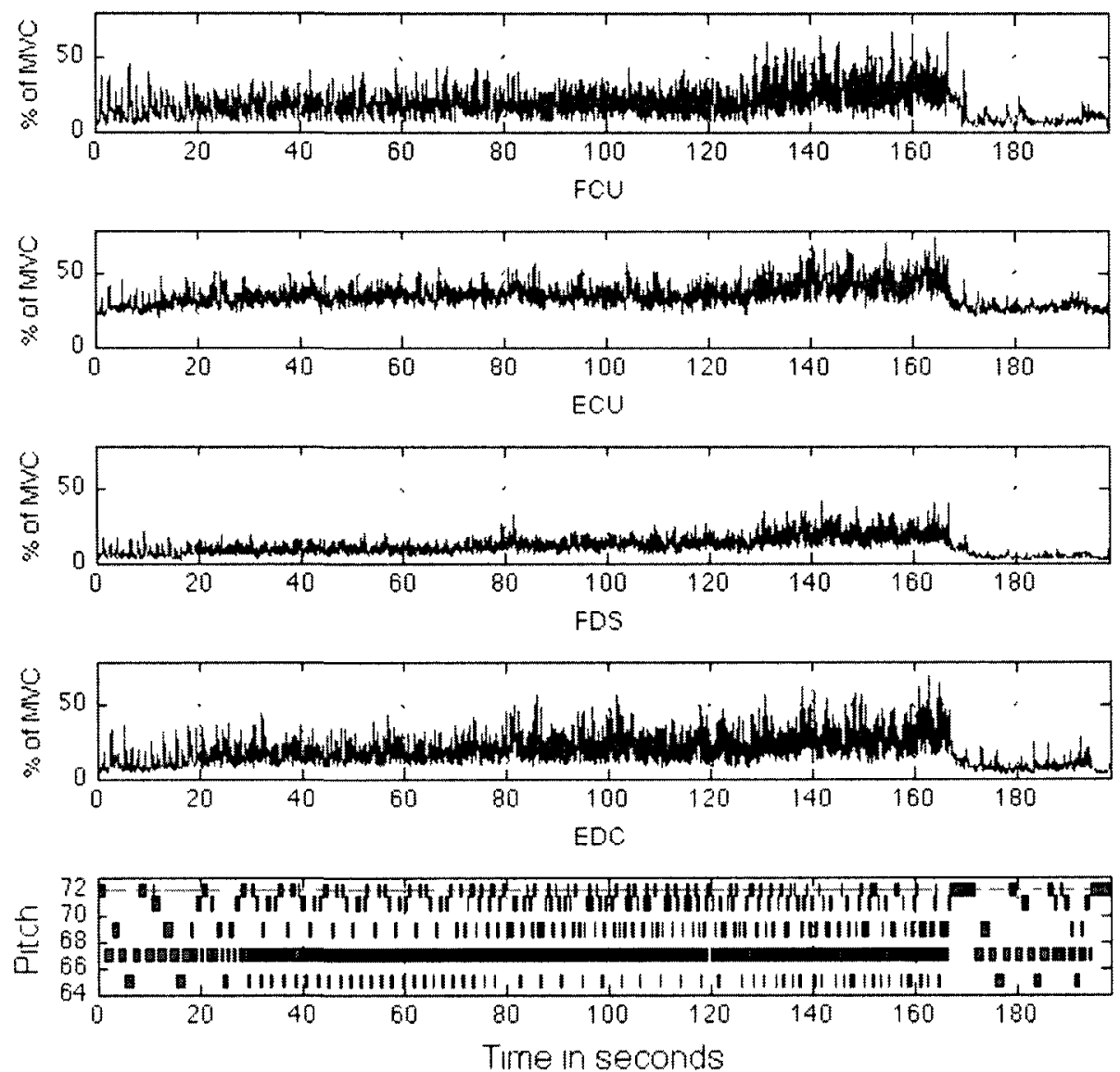

Figure O.1: Participant E002's EMG results: the upper plot shows active muscle stiffness, expressed as a percentage of maximal voluntary contraction, while playing the Ann Southam piece for the first time for four muscles of the right forearm: the FCU, the ECU, the FDS and the EDC. The corresponding MIDI data (note on, note off) data is shown on the lower plot. 

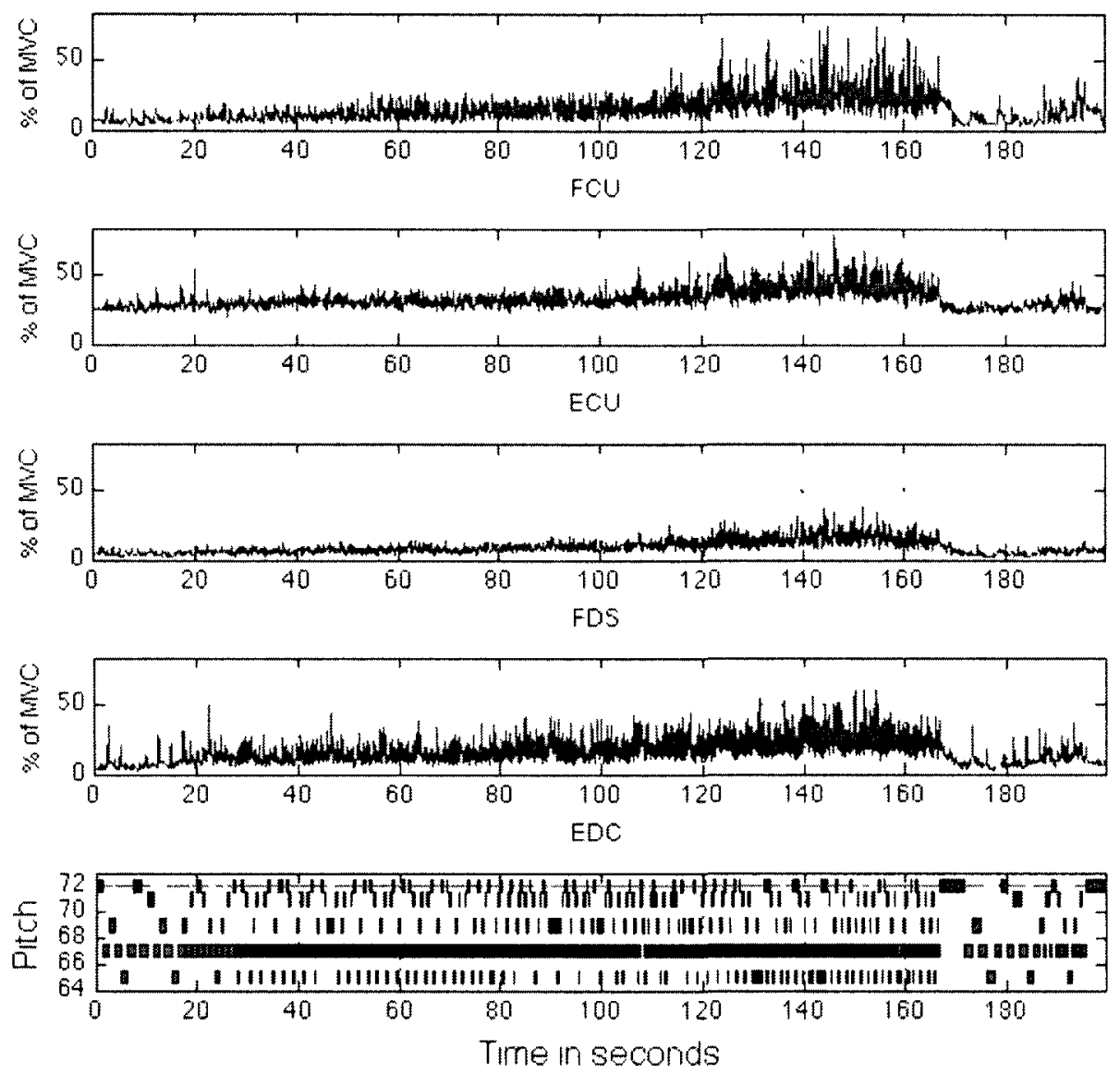

Figure O.2: Participant E002's EMG results: the upper plot shows active muscle stiffness, expressed as a percentage of maximal voluntary contraction, while playing the Ann Southam piece for the second time for four muscles of the right forearm: the FCU, the ECU, the FDS and the EDC. The corresponding MIDI data (note on, note off) data is shown on the lower plot. 

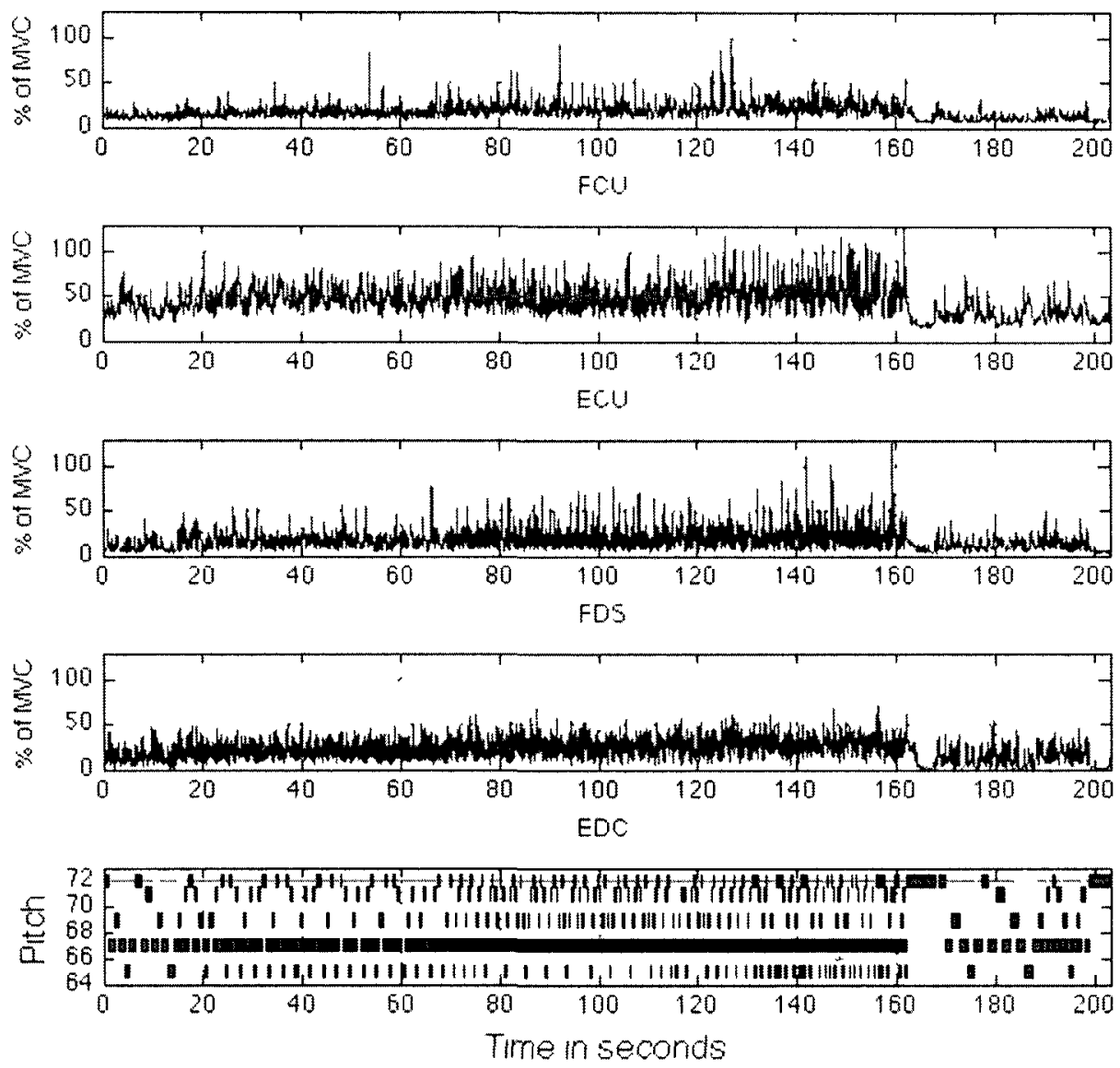

Figure O.3: Participant E003's EMG results: the upper plot shows active muscle stiffness, expressed as a percentage of maximal voluntary contraction, while playing the Ann Southam piece for the first time for four muscles of the right forearm: the FCU, the ECU, the FDS and the EDC. The corresponding MIDI data (note on, note off) data is shown on the lower plot. 

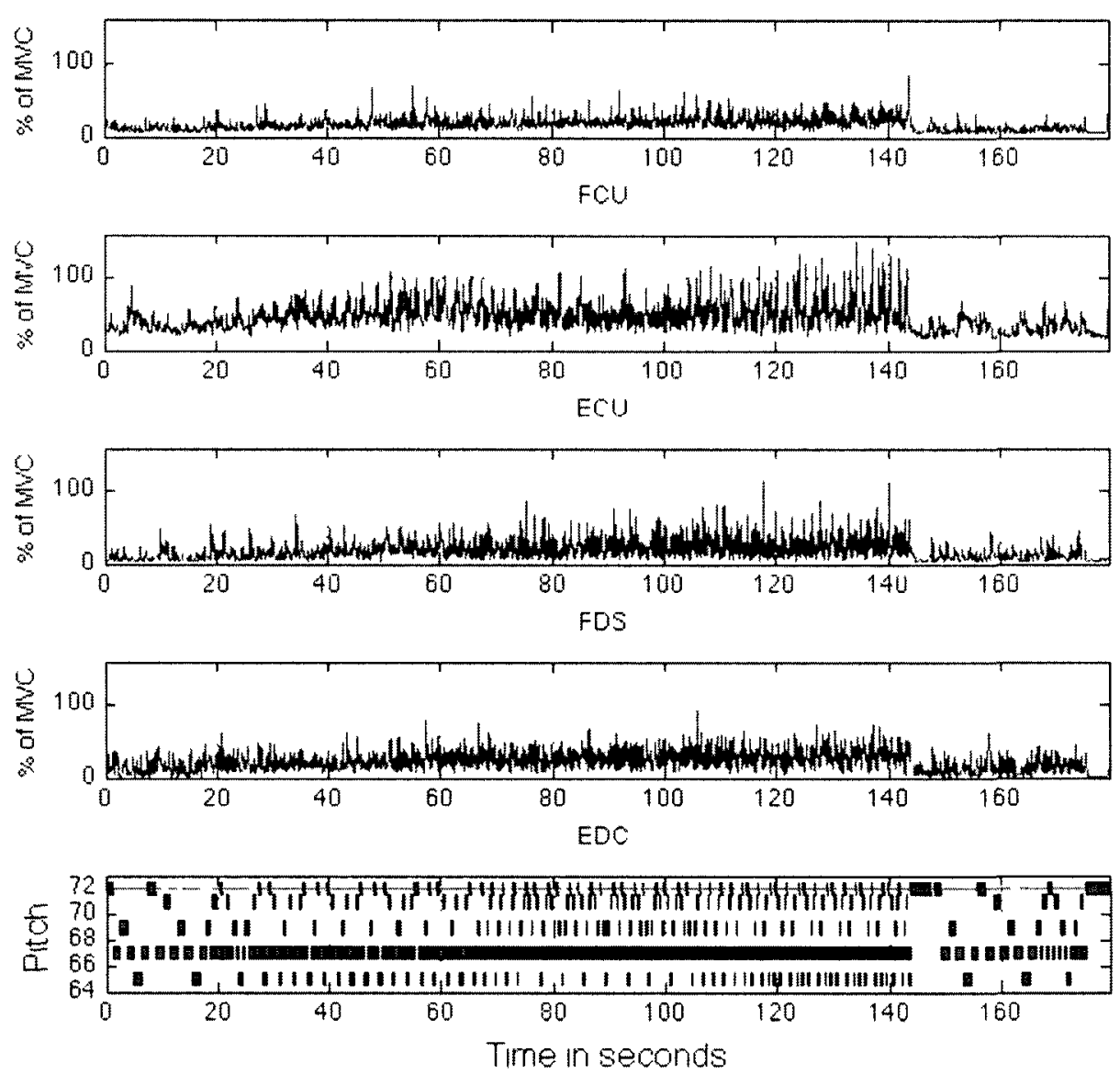

Figure O.4: Participant E003's EMG results: the upper plot shows active muscle stiffness, expressed as a percentage of maximal voluntary contraction, while playing the Ann Southam piece for the second time for four muscles of the right forearm: the FCU, the ECU, the FDS and the EDC. The corresponding MIDI data (note on, note off) data is shown on the lower plot. 

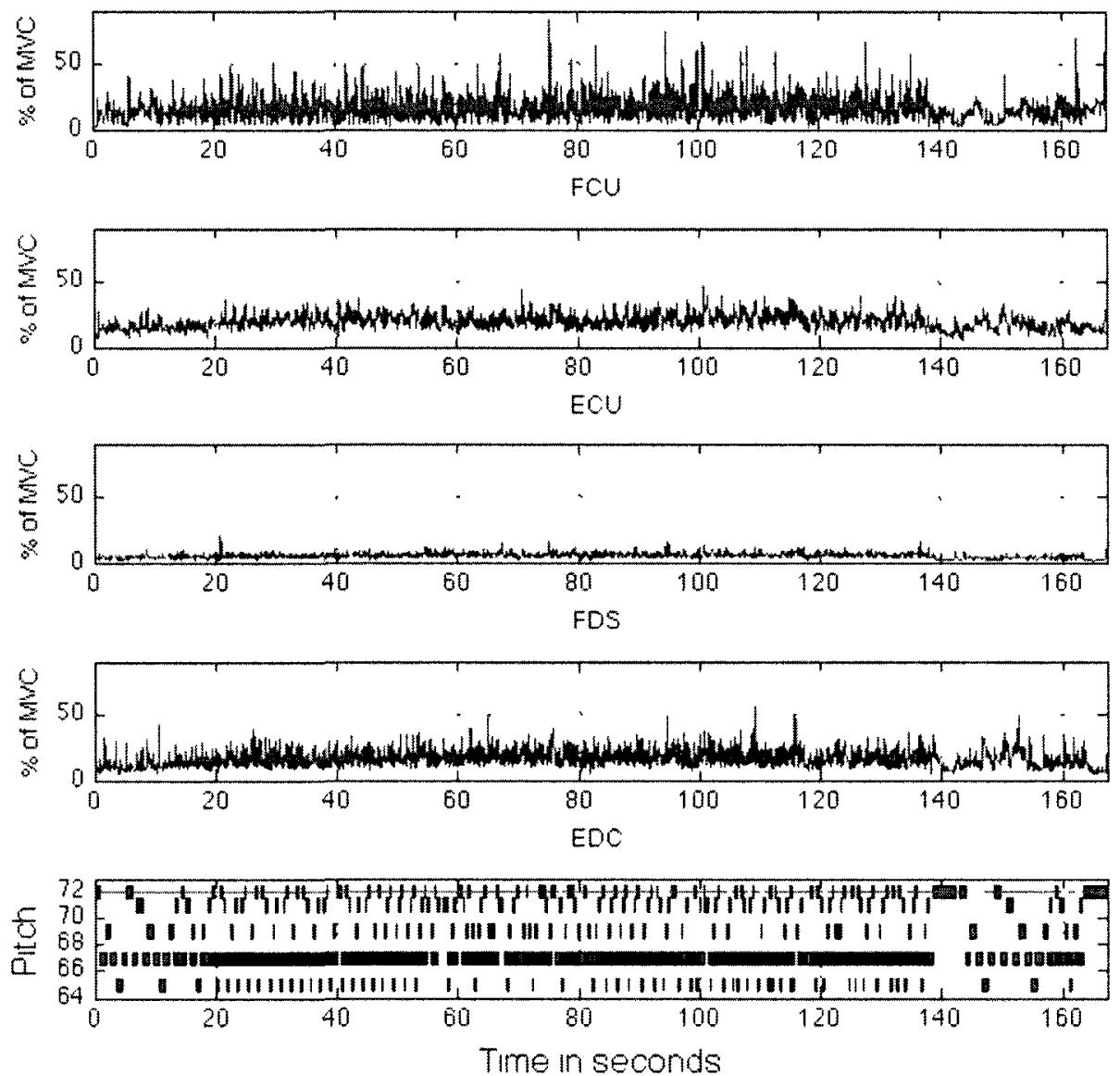

Figure O.5: Participant E004's EMG results: the upper plot shows active muscle stiffness, expressed as a percentage of maximal voluntary contraction, while playing the Ann Southam piece for the first time for four muscles of the right forearm: the FCU, the ECU, the FDS and the EDC. The corresponding MIDI data (note on, note off) data is shown on the lower plot. 

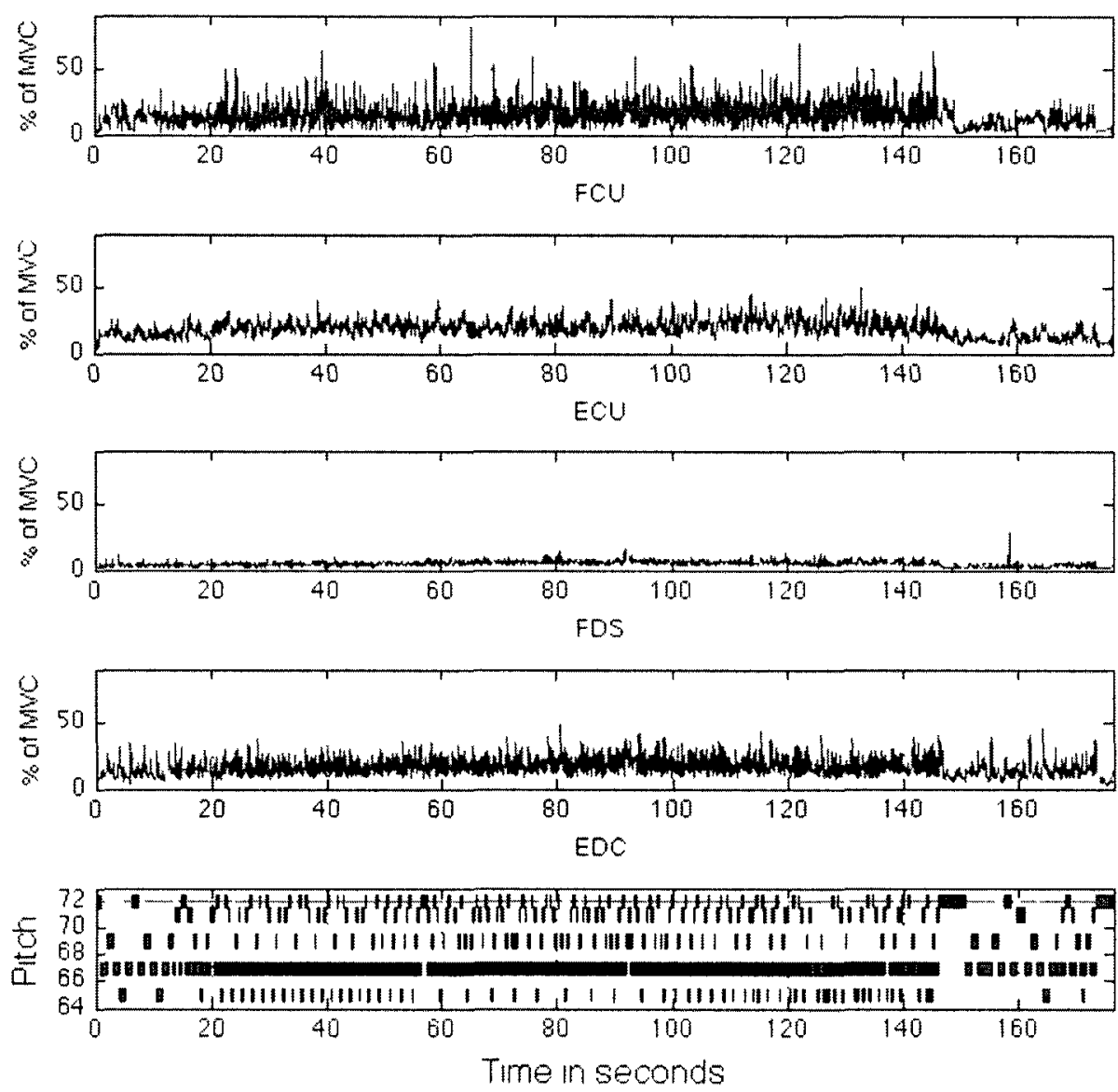

Figure O.6: Participant E004's EMG results: the upper plot shows active muscle stiffness, expressed as a percentage of maximal voluntary contraction, whlle playing the Ann Southam piece for the second time for four muscles of the right forearm: the FCU, the ECU, the FDS and the EDC. The corresponding MIDI data (note on, note off) data is shown on the lower plot. 

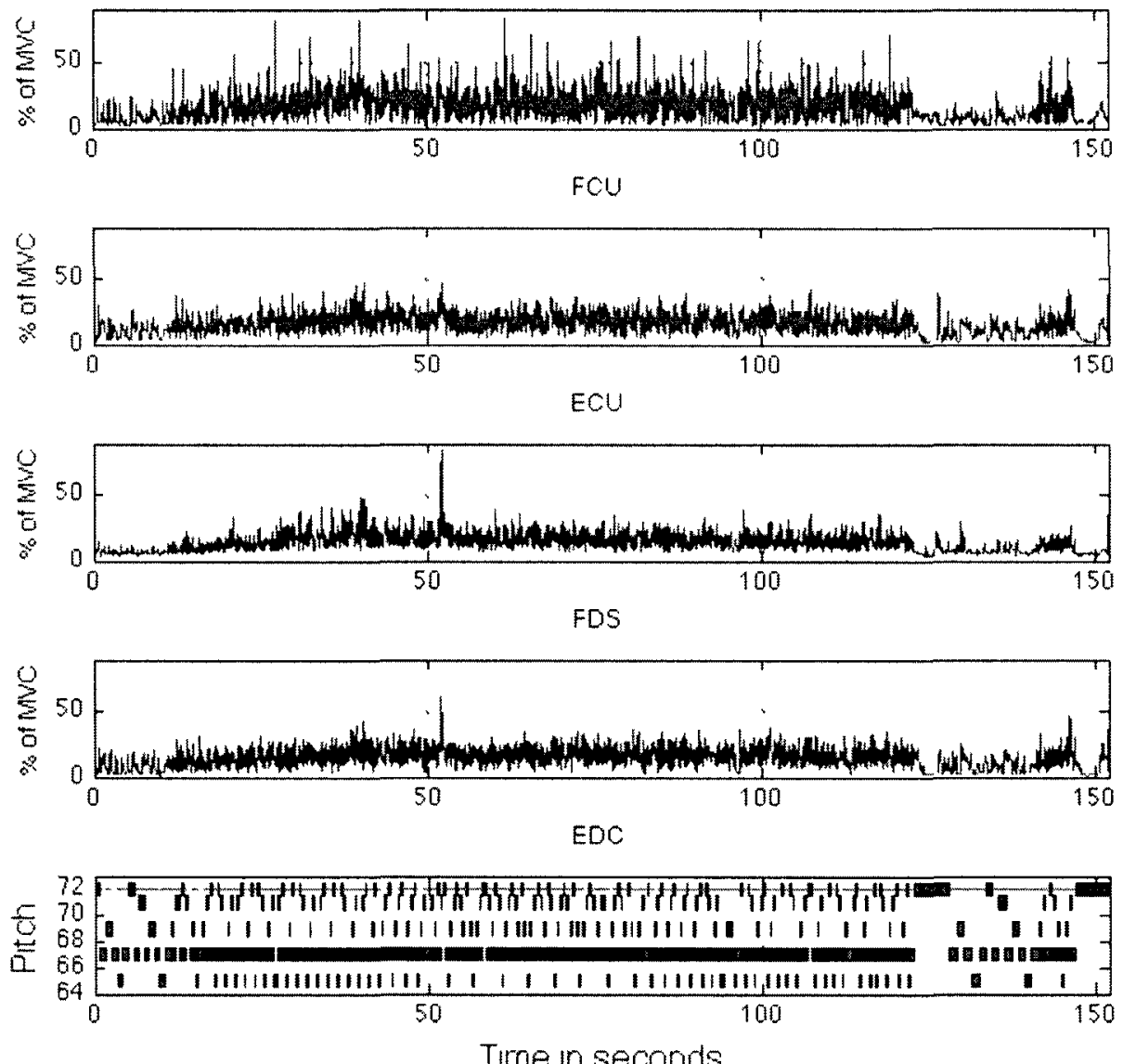

Figure O.7: Participant E005's EMG results: the upper plot shows active muscle stiffness, expressed as a percentage of maximal voluntary contraction, while playing the Ann Southam piece for the first time for four muscles of the right forearm: the FCU, the ECU, the FDS and the EDC. The corresponding MIDI data (note on, note off) data is shown on the lower plot. 

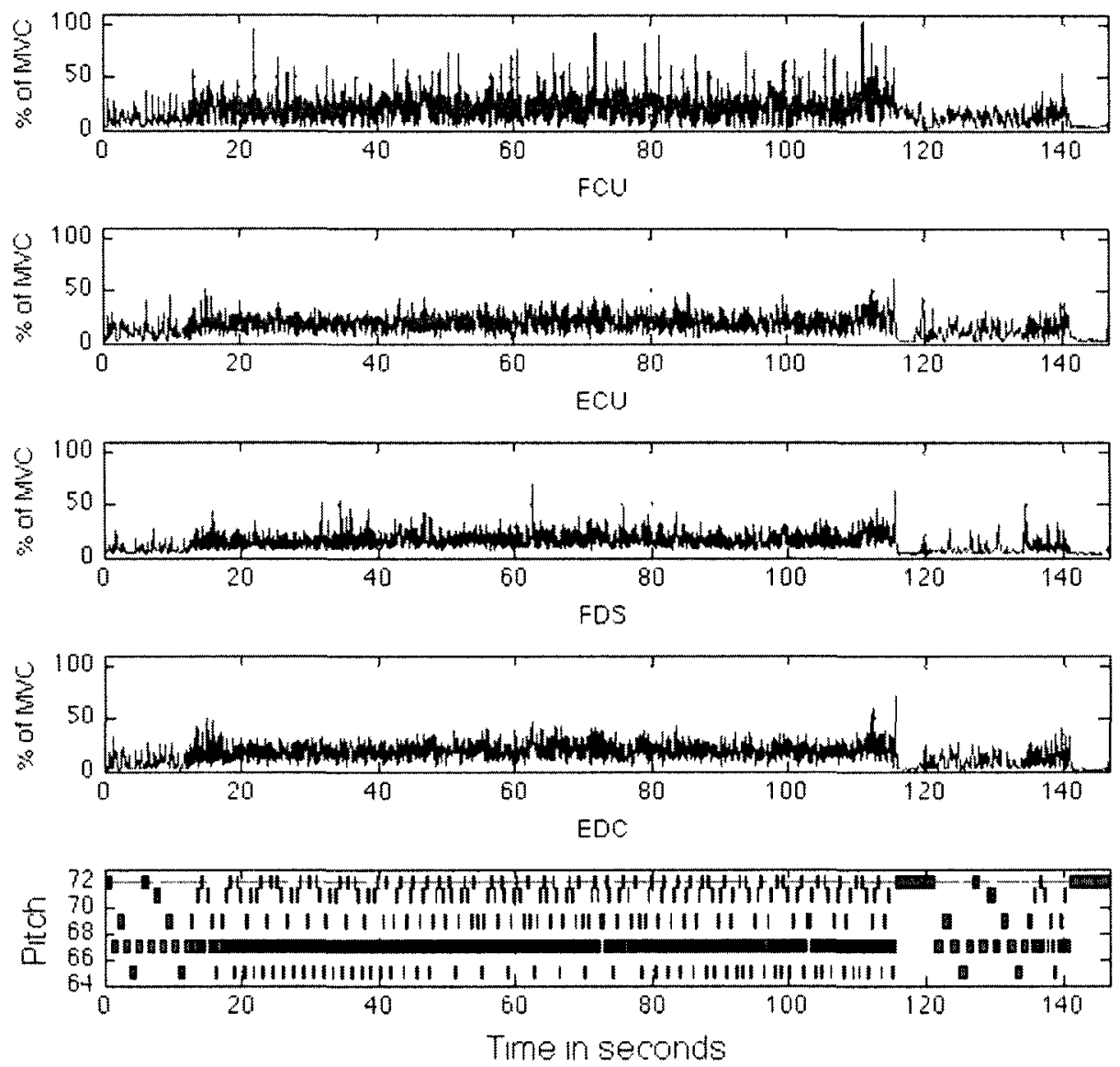

Figure O.8: Participant E005's EMG results: the upper plot shows active muscle stiffness, expressed as a percentage of maximal voluntary contraction, while playing the Ann Southam piece for the second time for four muscles of the right forearm: the FCU, the ECU, the FDS and the EDC. The corresponding MIDI data (note on, note off) data is shown on the lower plot. 

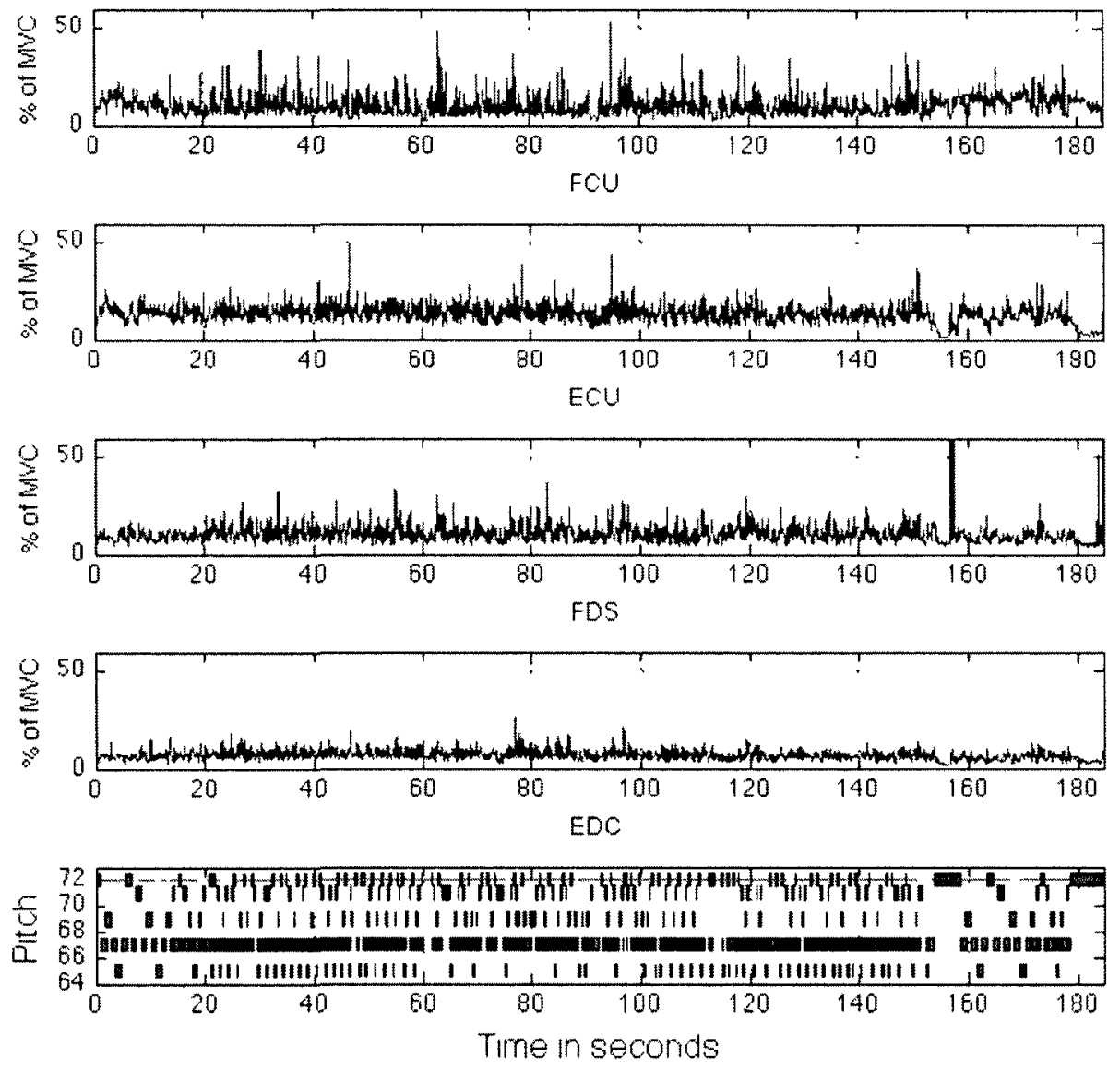

Figure O.9: Participant E006's EMG results: the upper plot shows active muscle stiffness, expressed as a percentage of maximal voluntary contraction, while playing the Ann Southam piece for the first time for four muscles of the right forearm: the FCU, the ECU, the FDS and the EDC. The corresponding MIDI data (note on, note off) data is shown on the lower plot. 

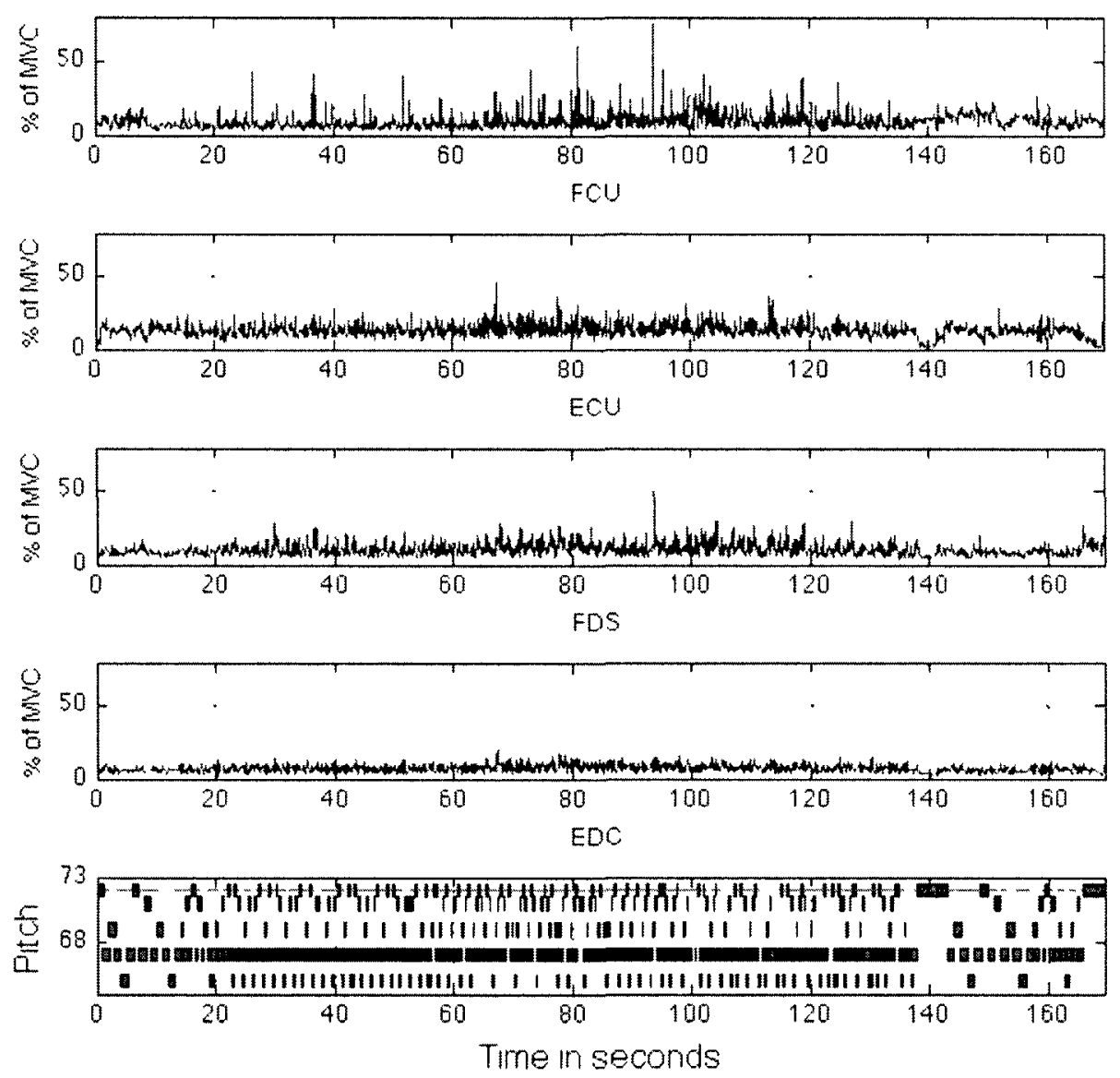

Figure O.10: Participant E006's EMG results: the upper plot shows active muscle stiffness, expressed as a percentage of maximal voluntary contraction, while playing the Ann Southam piece for the second time for four muscles of the right forearm: the FCU, the ECU, the FDS and the EDC. The corresponding MIDI data (note on, note off) data is shown on the lower plot. 

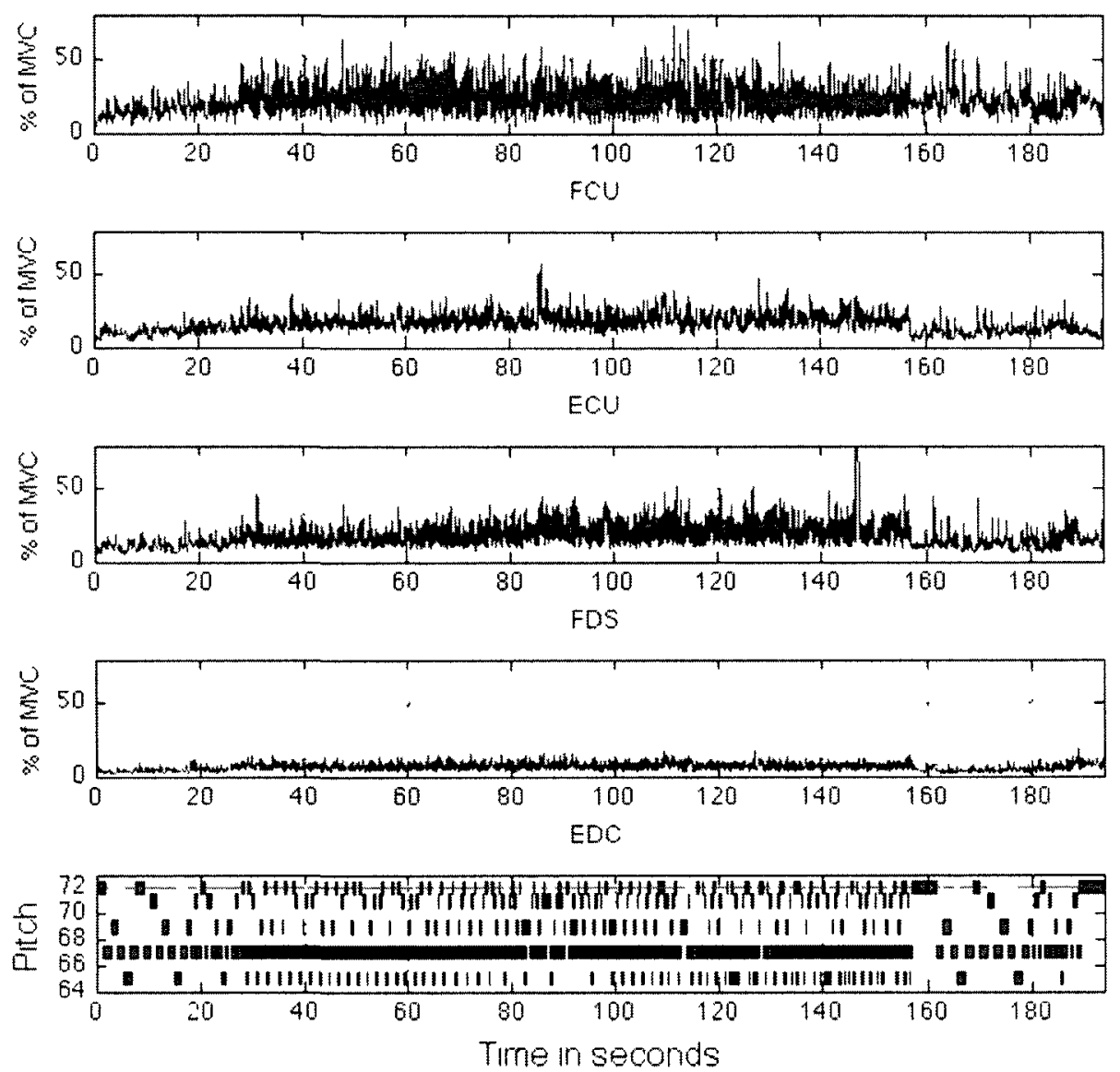

Figure O.11: Participant E007's EMG results: the upper plot shows active muscle stiffness, expressed as a percentage of maximal voluntary contraction, while playing the Ann Southam piece for the first time for four muscles of the right forearm: the FCU, the ECU, the FDS and the EDC. The corresponding MIDI data (note on, note off) data is shown on the lower plot. 

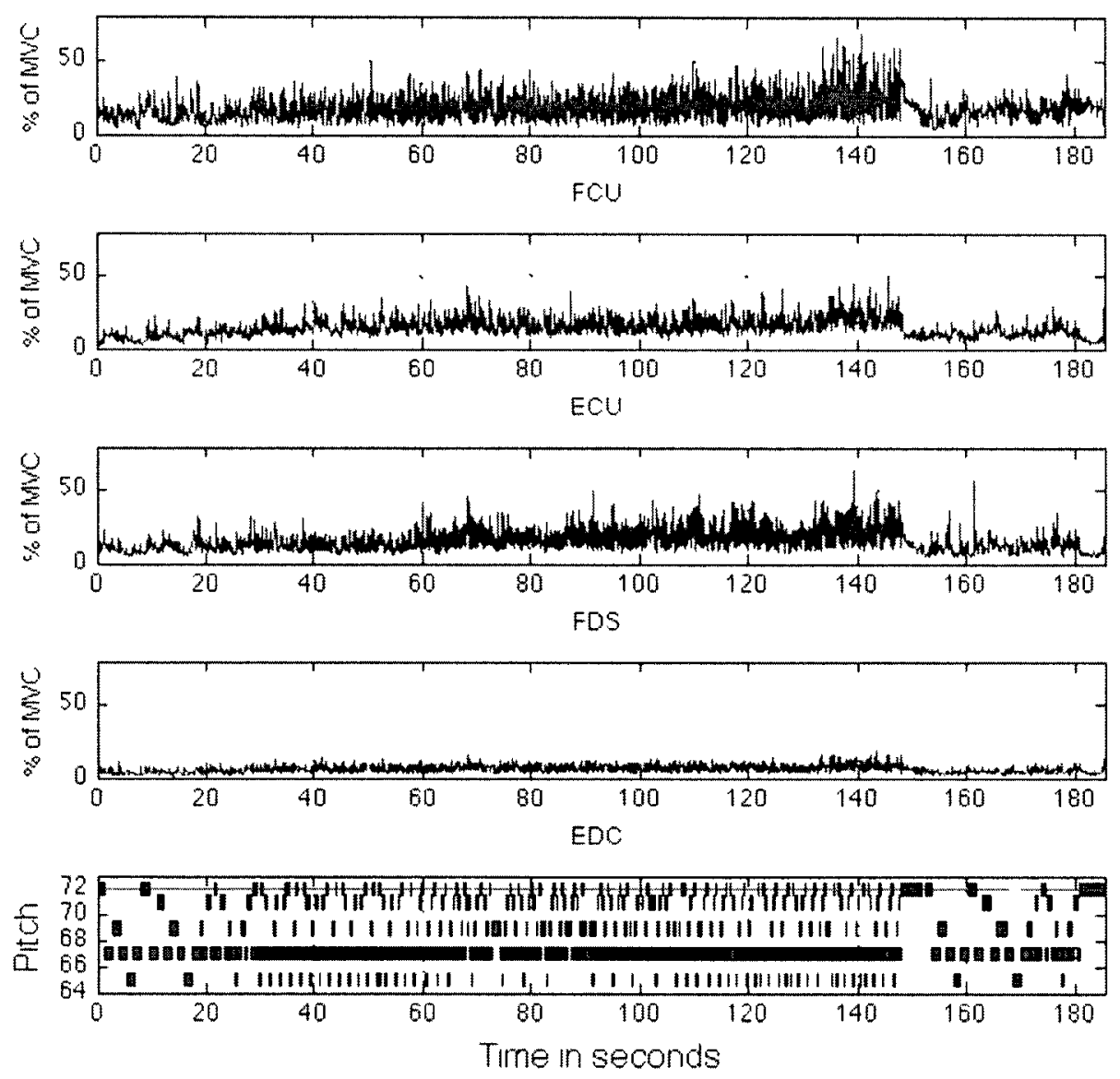

Figure O.12: Participant E007's EMG results: the upper plot shows active muscle stiffness, expressed as a percentage of maximal voluntary contraction, while playing the Ann Southam piece for the second time for four muscles of the right forearm: the FCU, the ECU, the FDS and the EDC. The corresponding MIDI data (note on, note off) data is shown on the lower plot. 

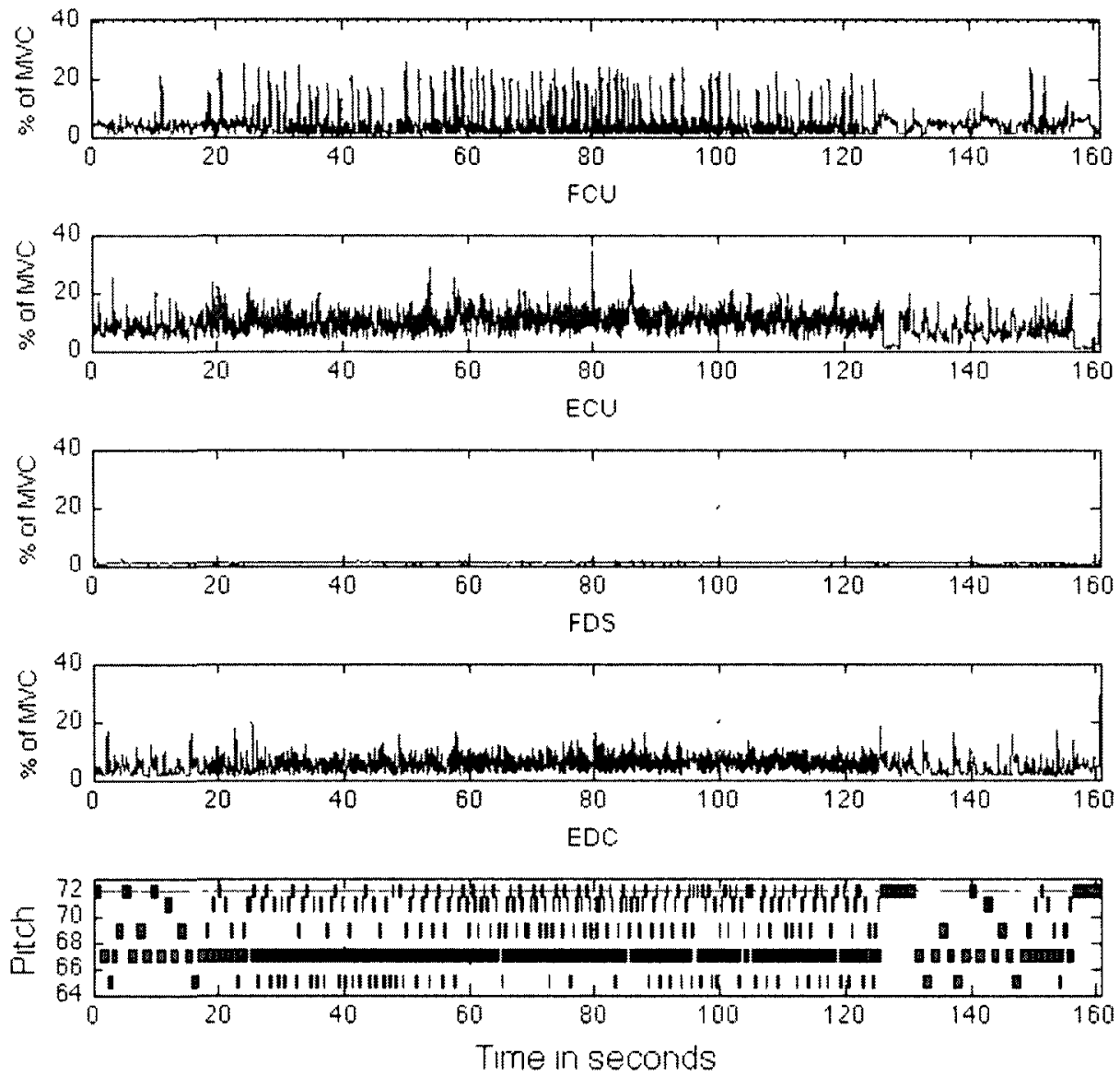

Figure O.13: Participant E008's EMG results: the upper plot shows active muscle stiffness, expressed as a percentage of maximal voluntary contraction, while playing the Ann Southam piece for the first time for four muscles of the right forearm: the FCU, the ECU, the FDS and the EDC. The corresponding MIDI data (note on, note off) data is shown on the lower plot. 

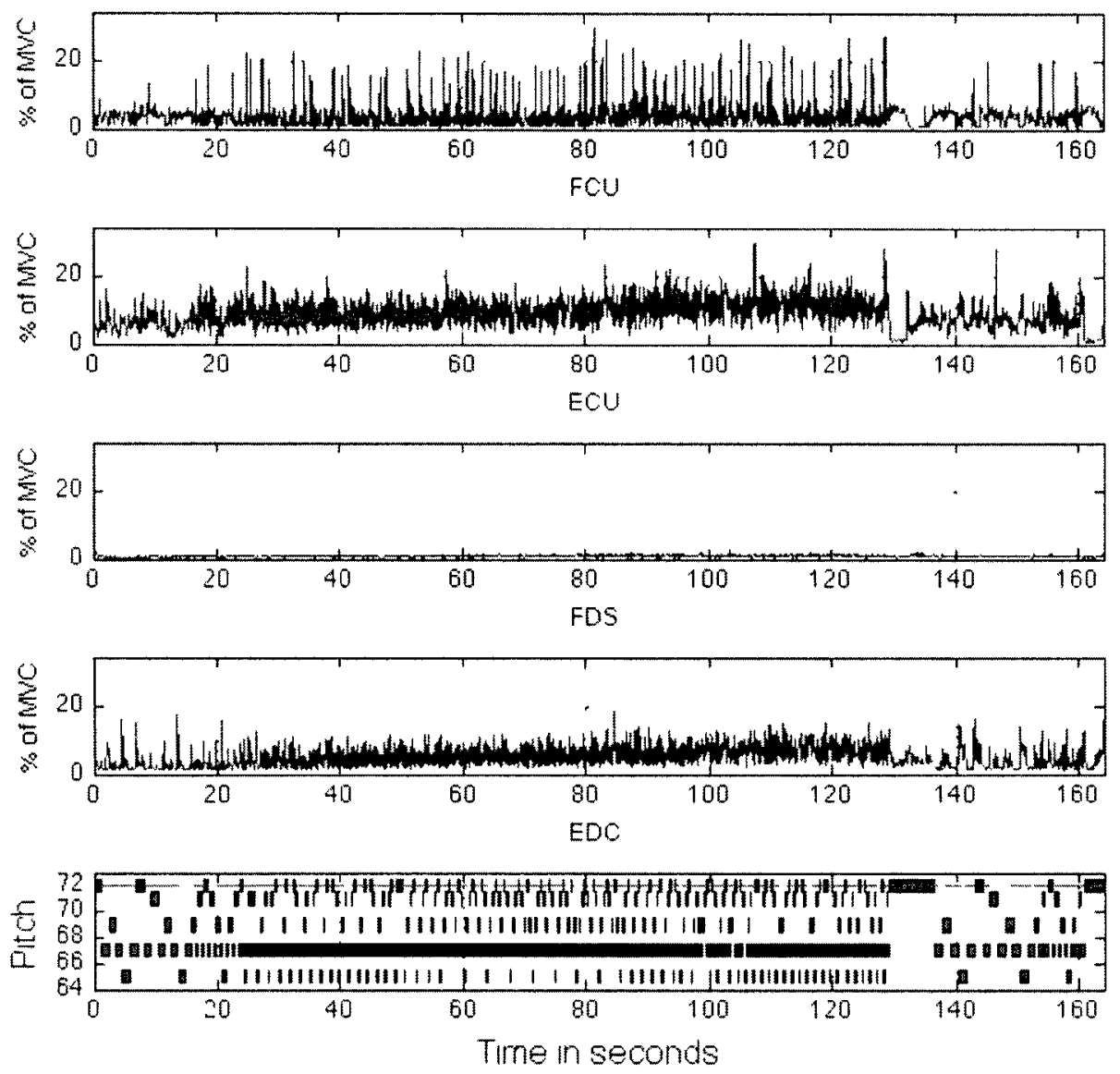

Figure O.14: Participant E008's EMG results: the upper plot shows active muscle stiffness, expressed as a percentage of maximal voluntary contraction, while playing the Ann Southam piece for the second time for four muscles of the right forearm: the FCU, the ECU, the FDS and the EDC. The corresponding MIDI data (note on, note off) data is shown on the lower plot 

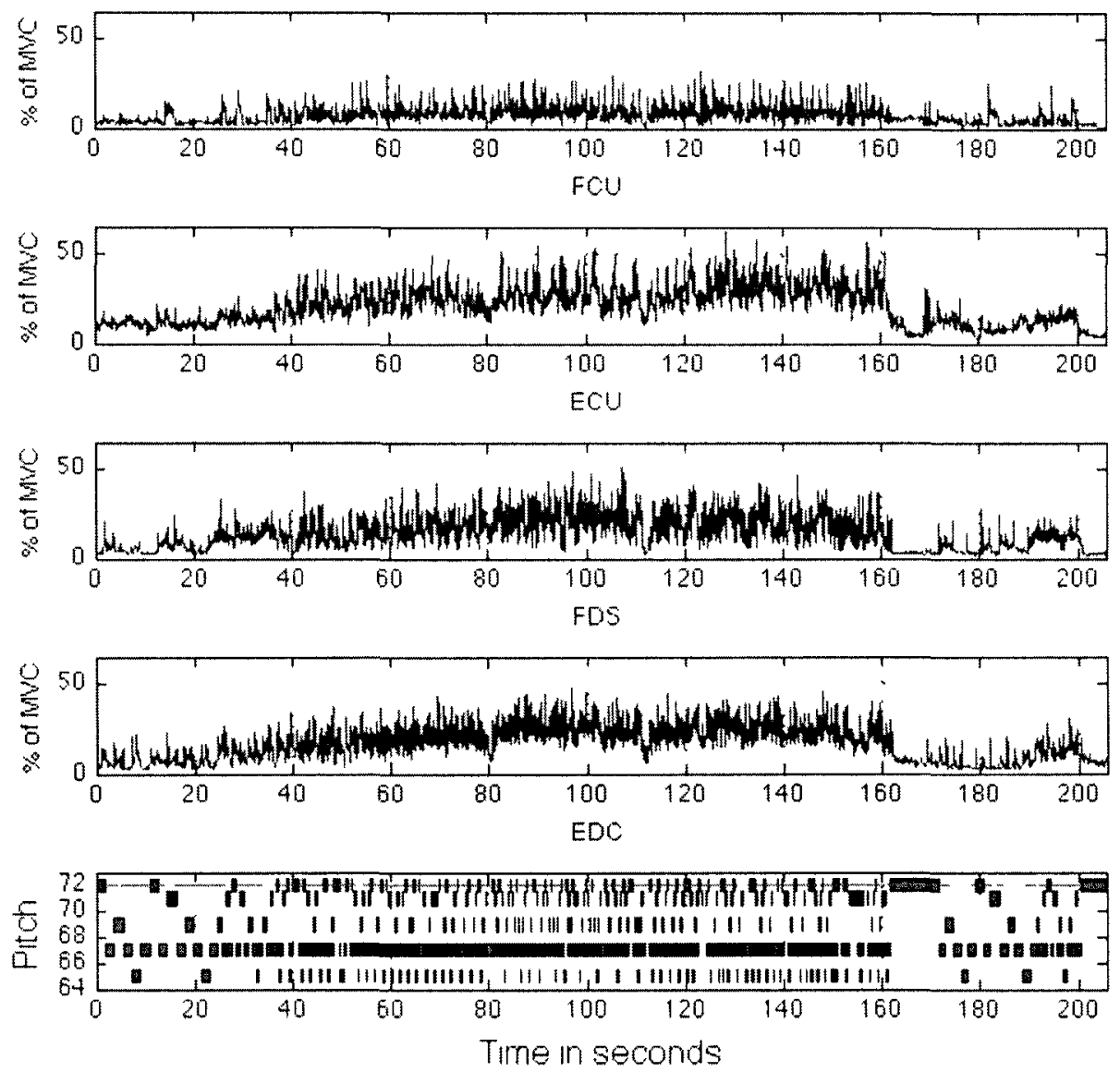

Figure 0.15: Participant E009's EMG results: the upper plot shows active muscle stiffness, expressed as a percentage of maximal voluntary contraction, while playing the Ann Southam piece for the first time for four muscles of the right forearm: the FCU, the ECU, the FDS and the EDC. The corresponding MIDI data (note on, note off) data is shown on the lower plot. 

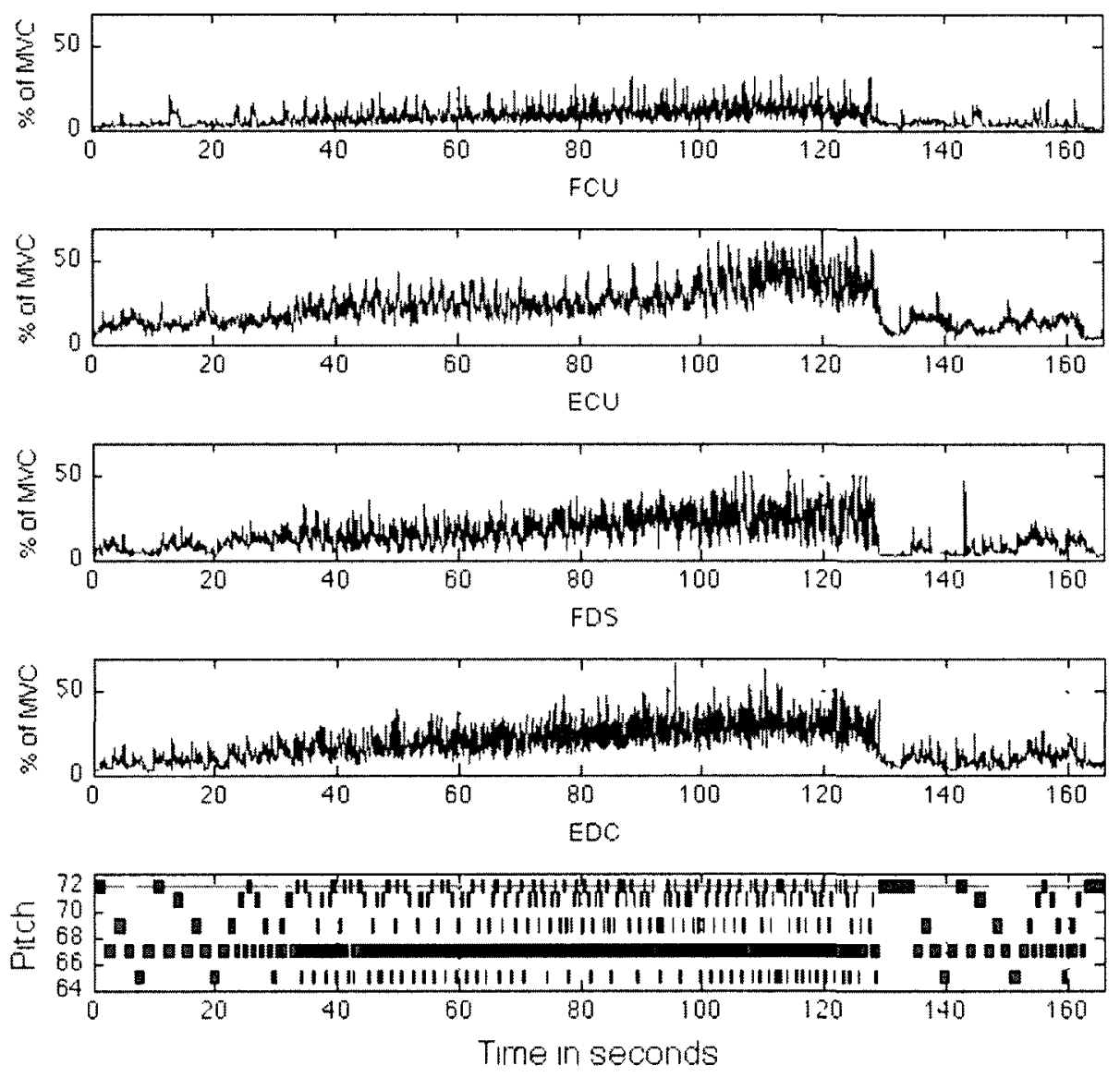

Figure O.16: Participant E009's EMG results: the upper plot shows active muscle stiffness, expressed as a percentage of maximal voluntary contraction, while playing the Ann Southam piece for the second time for four muscles of the right forearm: the FCU, the ECU, the FDS and the EDC. The corresponding MIDI data (note on, note off) data is shown on the lower plot. 

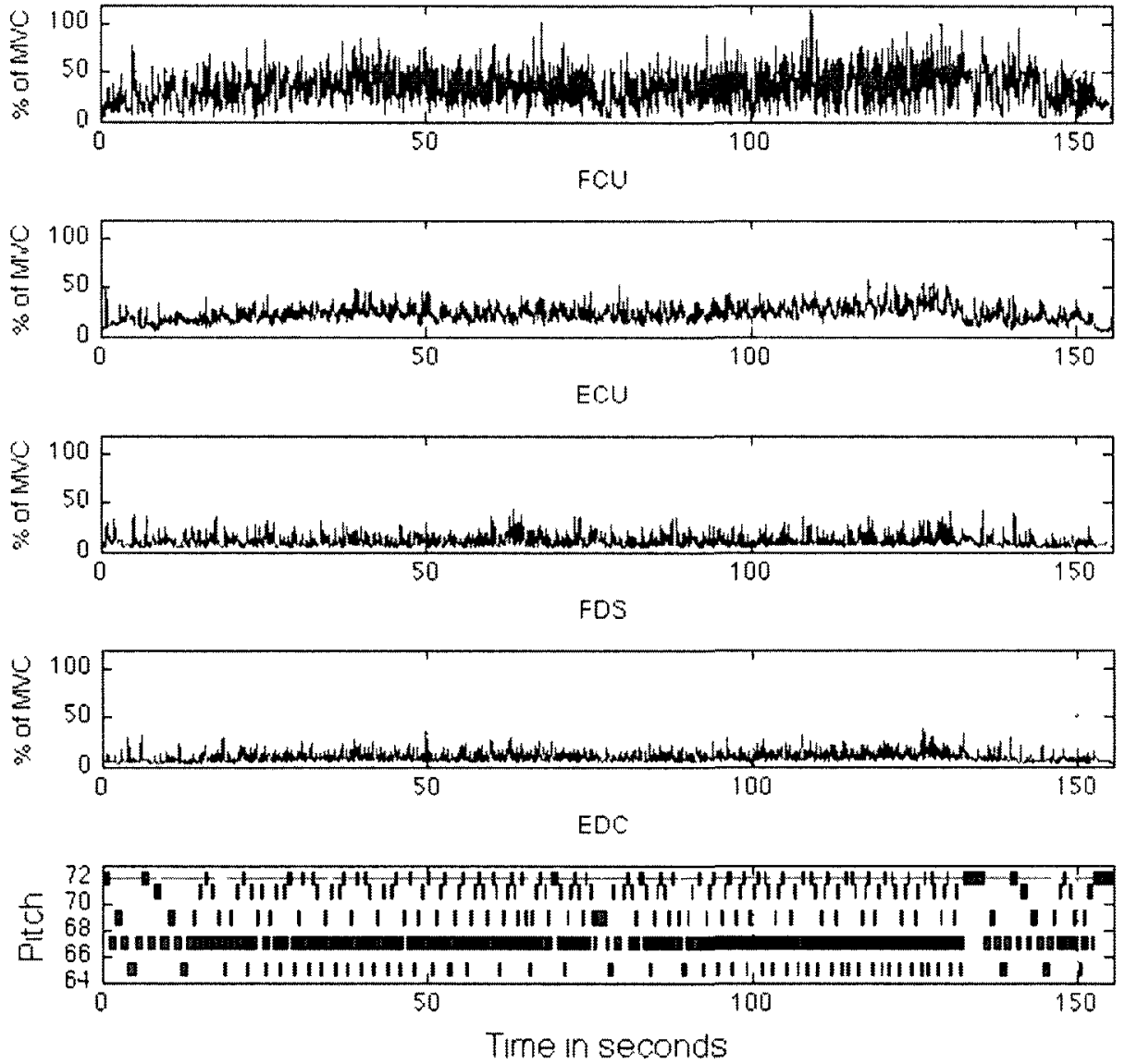

Figure O.17: Participant E010's EMG results: the upper plot shows active muscle stiffness, expressed as a percentage of maximal voluntary contraction, while playing the Ann Southam piece for the first time for four muscles of the right forearm: the FCU, the ECU, the FDS and the EDC. The corresponding MIDI data (note on, note off) data is shown on the lower plot. 

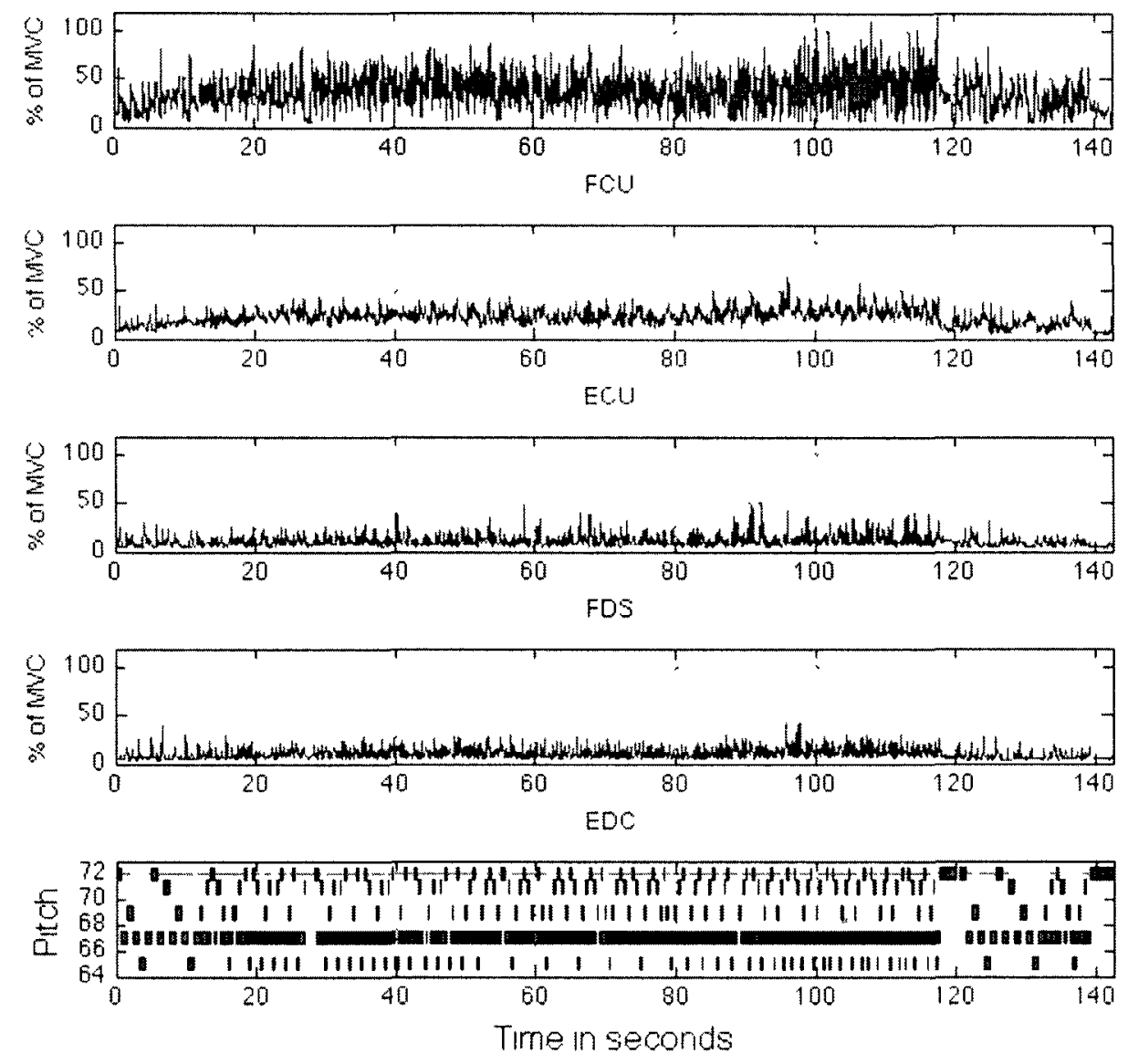

Figure O.18: Participant E010's EMG results: the upper plot shows active muscle stiffness, expressed as a percentage of maximal voluntary contraction, while playing the Ann Southam piece for the second time for four muscles of the right forearm: the FCU, the ECU, the FDS and the EDC. The corresponding MIDI data (note on, note off) data is shown on the lower plot. 
Appendix $\mathbf{P}$

Plots of Participants' Co-contraction

Levels and MIDI Data While Playing the

Piece Composed by Ann Southam 

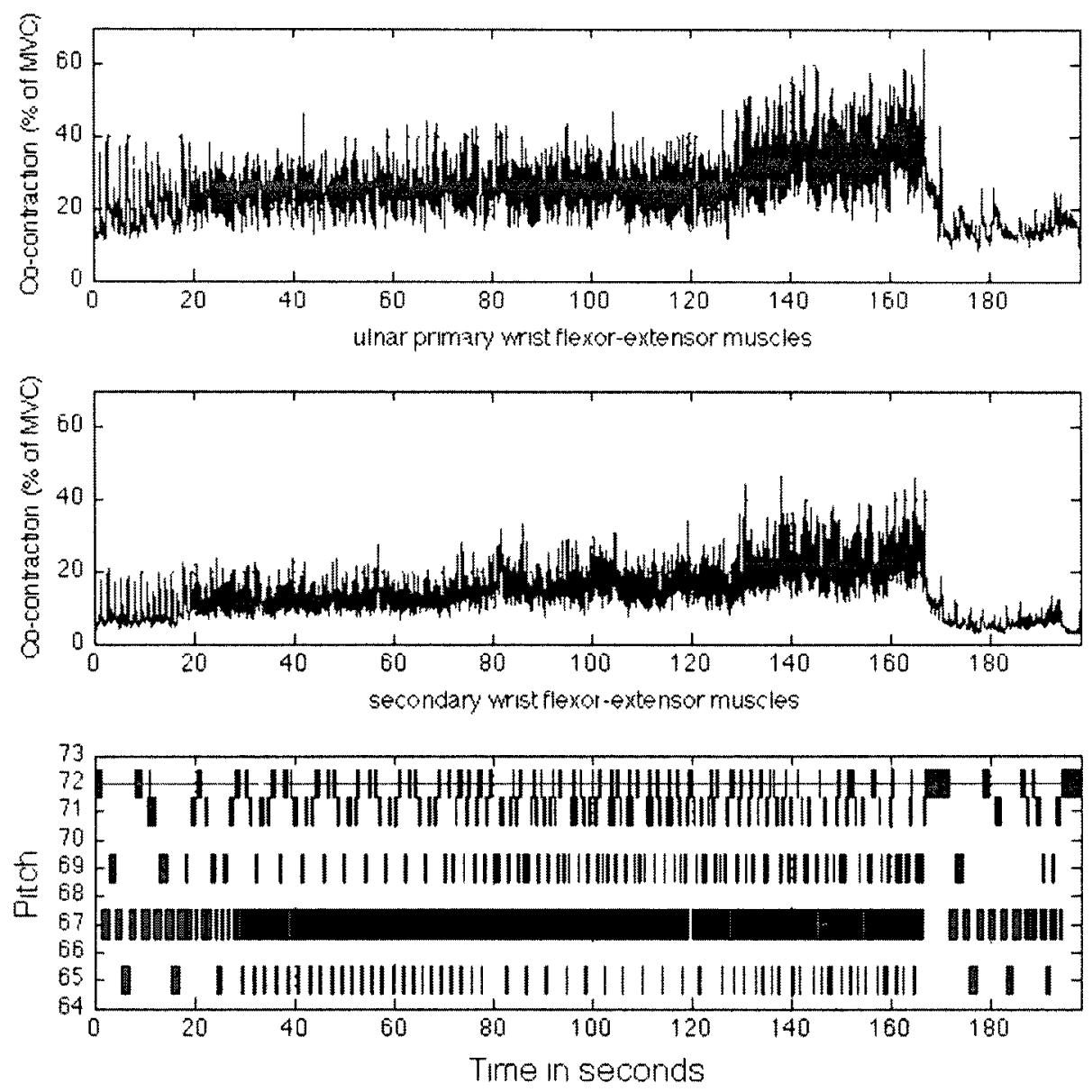

Figure P.1: Participant E002's EMG results: the upper plot shows levels of muscular co-contraction, expressed as a percentage of maximal voluntary contraction, whlle playing the Ann Southam piece for the first time for four muscles of the right forearm: the FCU, the ECU, the FDS and the EDC. The corresponding MIDI data (note on, note off) data is shown on the lower plot. 

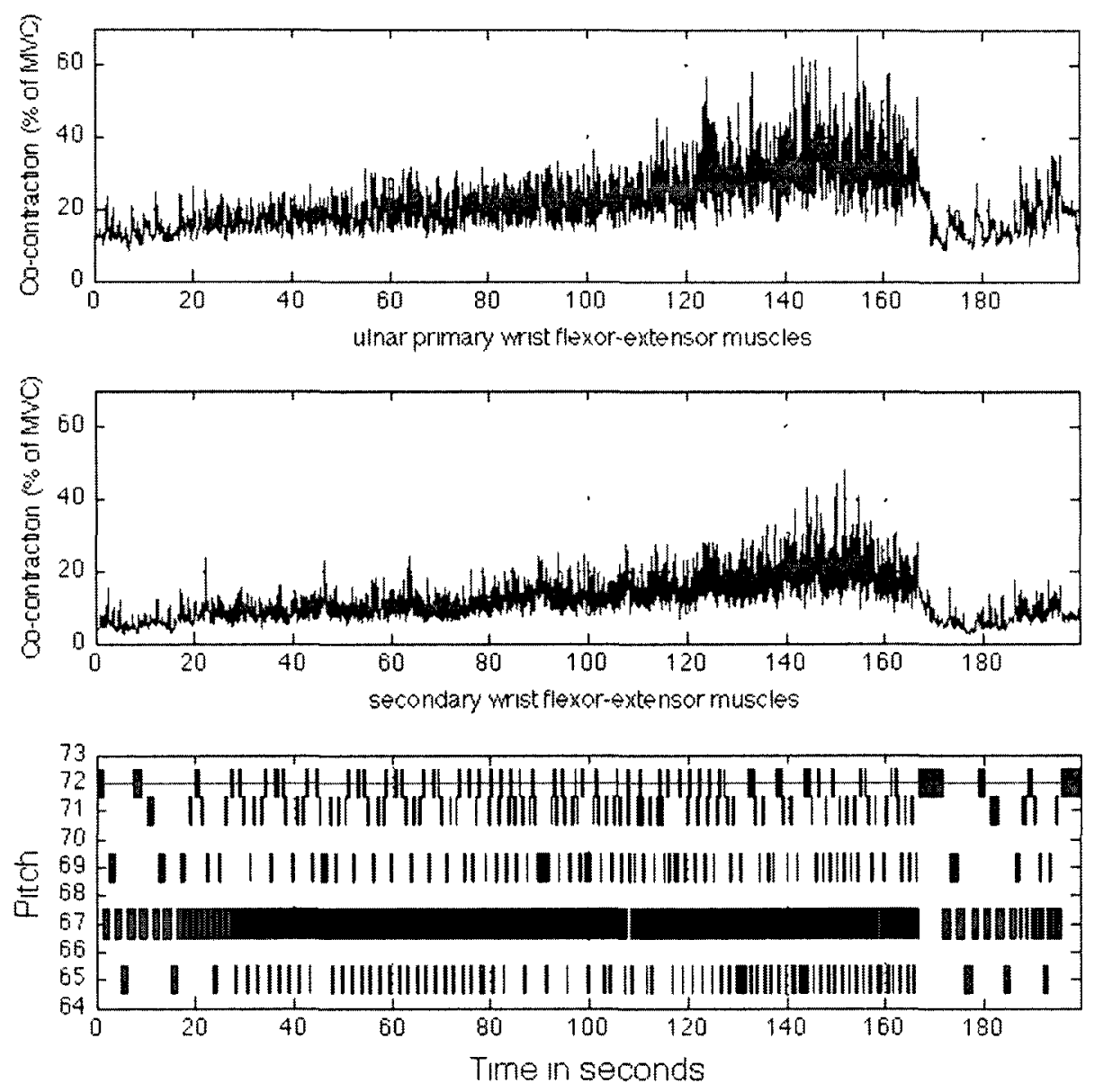

Figure P.2: Participant E002's EMG results: the upper plot shows levels of muscular co-contraction, expressed as a percentage of maximal voluntary contraction, while playing the Ann Southam piece for the second time for four muscles of the right forearm: the FCU, the ECU, the FDS and the EDC. The corresponding MIDI data (note on, note off) data is shown on the lower plot. 

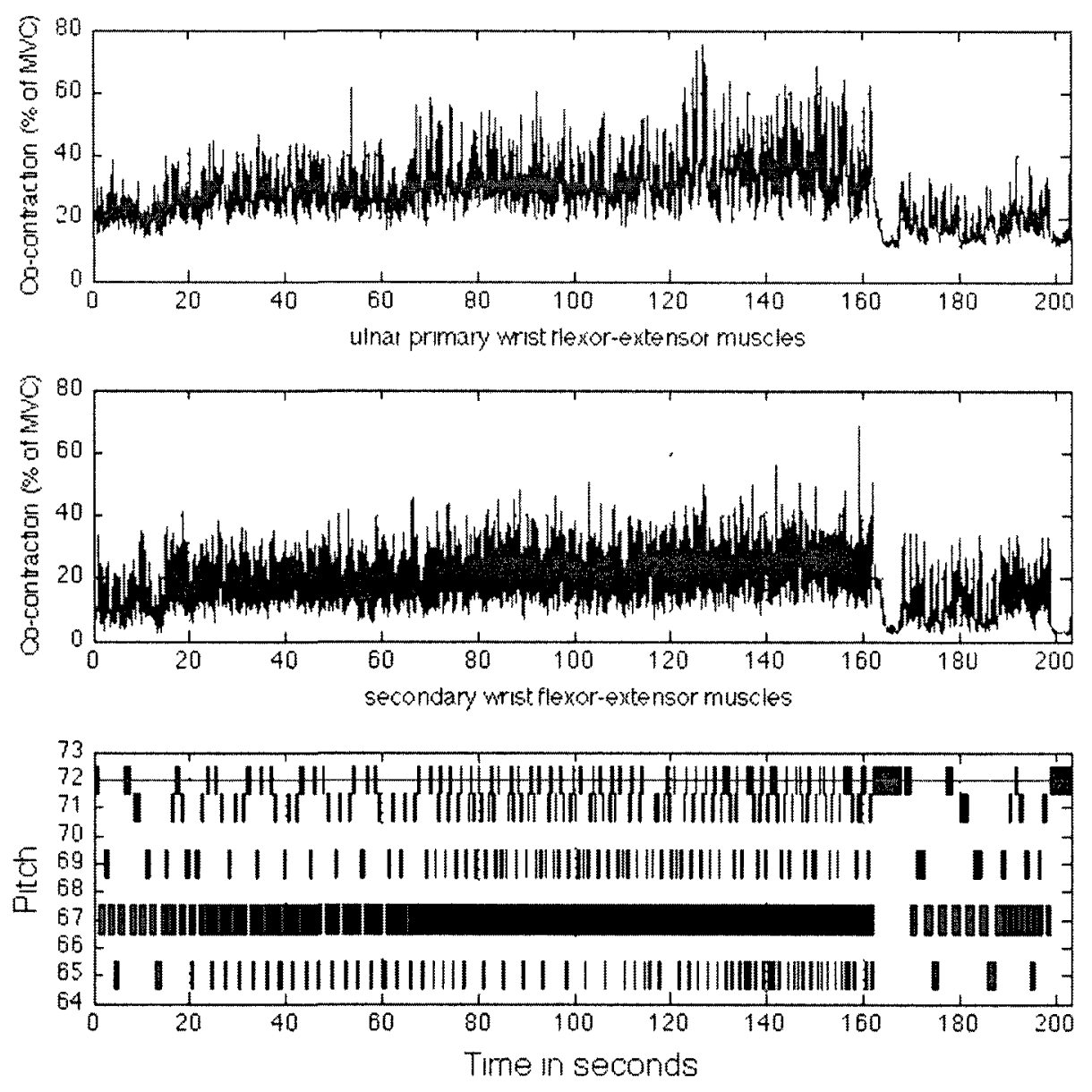

Figure P.3: Participant E003's EMG results' the upper plot shows levels of muscular co-contraction, expressed as a percentage of maximal voluntary contraction, while playing the Ann Southam piece for the first time for four muscles of the right forearm the FCU, the ECU, the FDS and the EDC. The corresponding MIDI data (note on, note off) data is shown on the lower plot. 

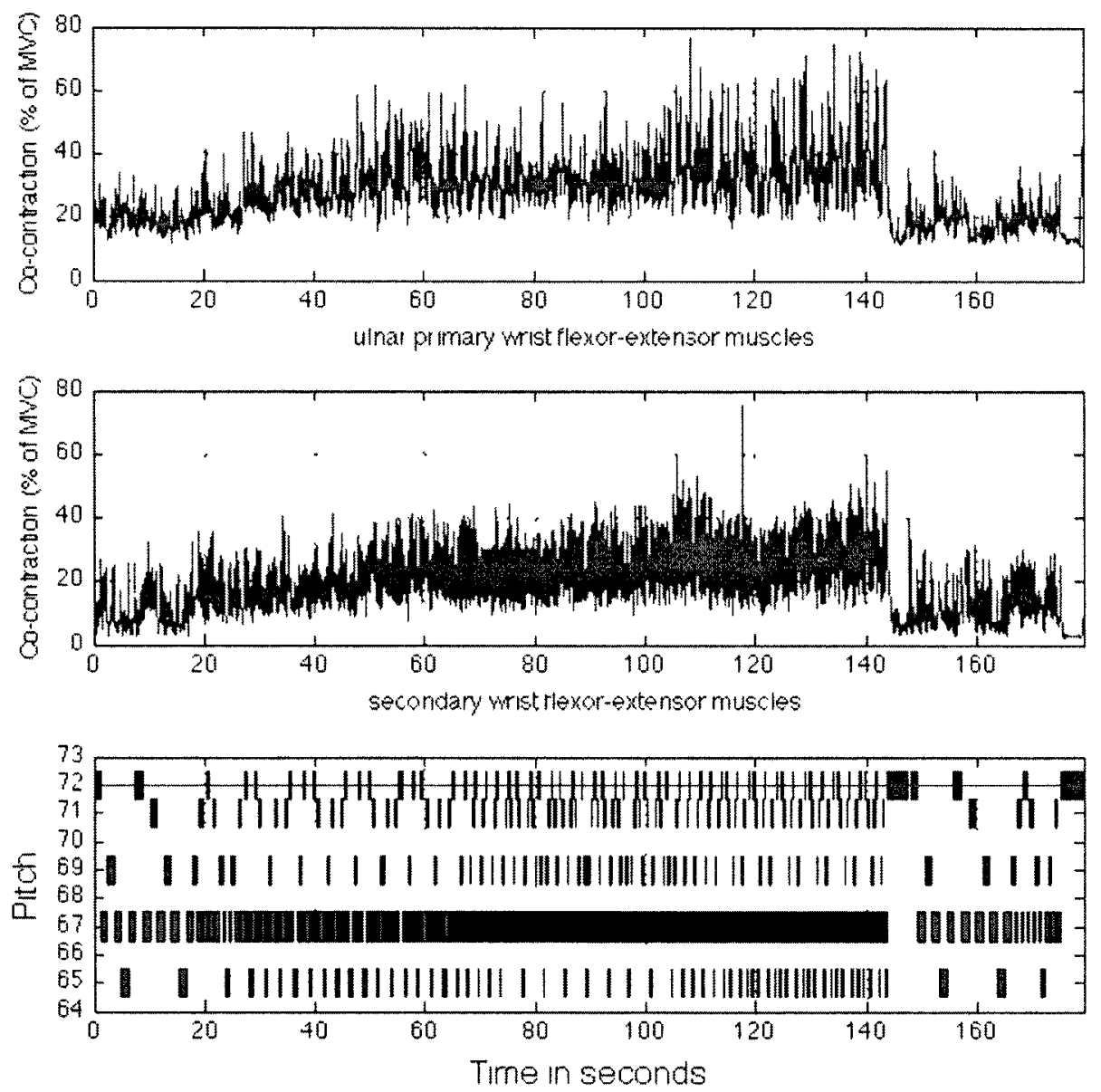

Figure P.4: Participant E003's EMG results: the upper plot shows levels of muscular co-contraction, expressed as a percentage of maximal voluntary contraction, while playing the Ann Southam piece for the second time for four muscles of the right forearm: the FCU, the ECU, the FDS and the EDC. The corresponding MIDI data (note on, note off) data is shown on the lower plot. 

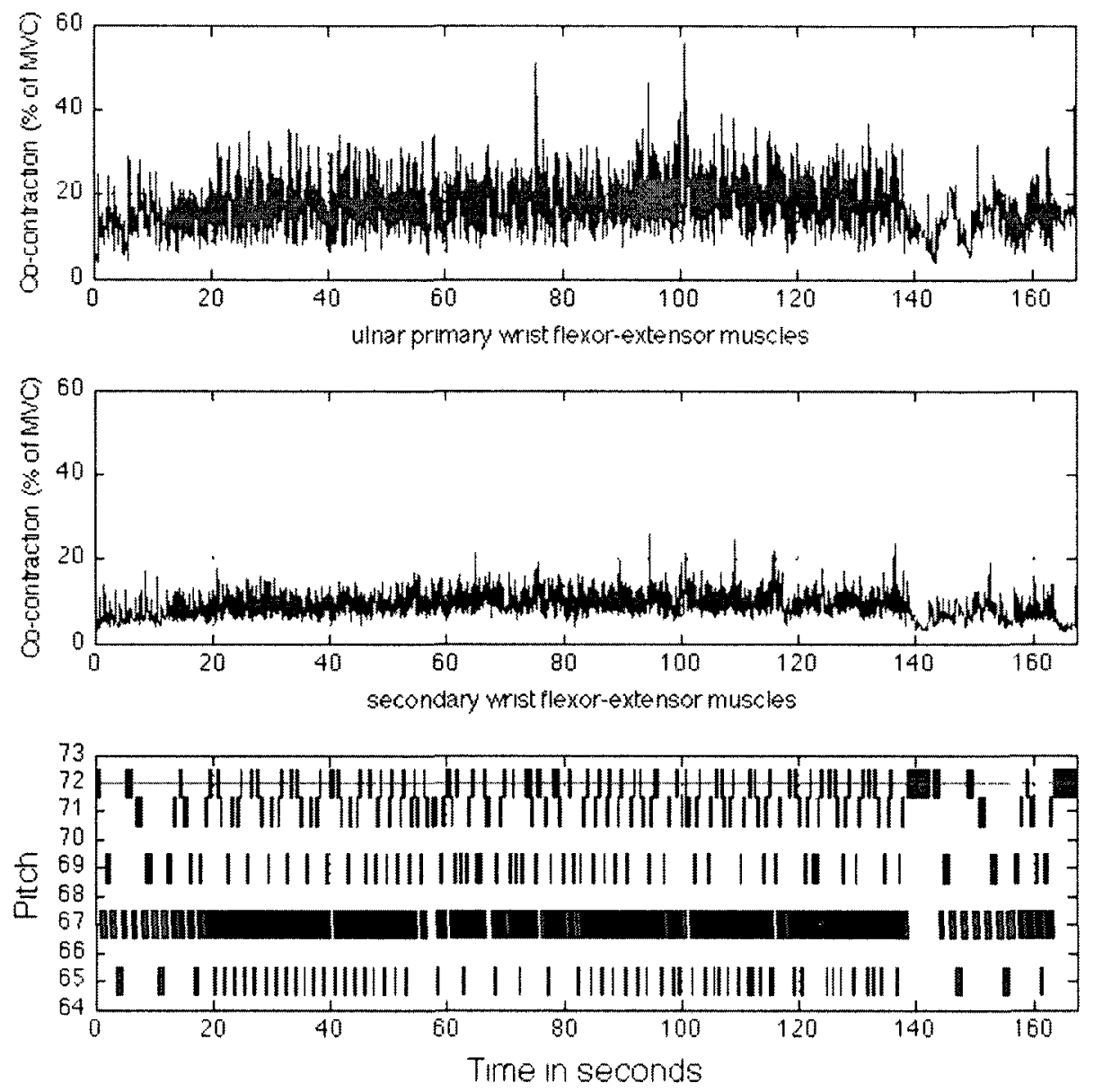

Figure P.5: Participant E004's EMG results: the upper plot shows levels of muscular co-contraction, expressed as a percentage of maximal voluntary contraction, while playing the Ann Southam piece for the first time for four muscles of the right forearm: the FCU, the ECU, the FDS and the EDC. The corresponding MIDI data (note on, note off) data is shown on the lower plot. 

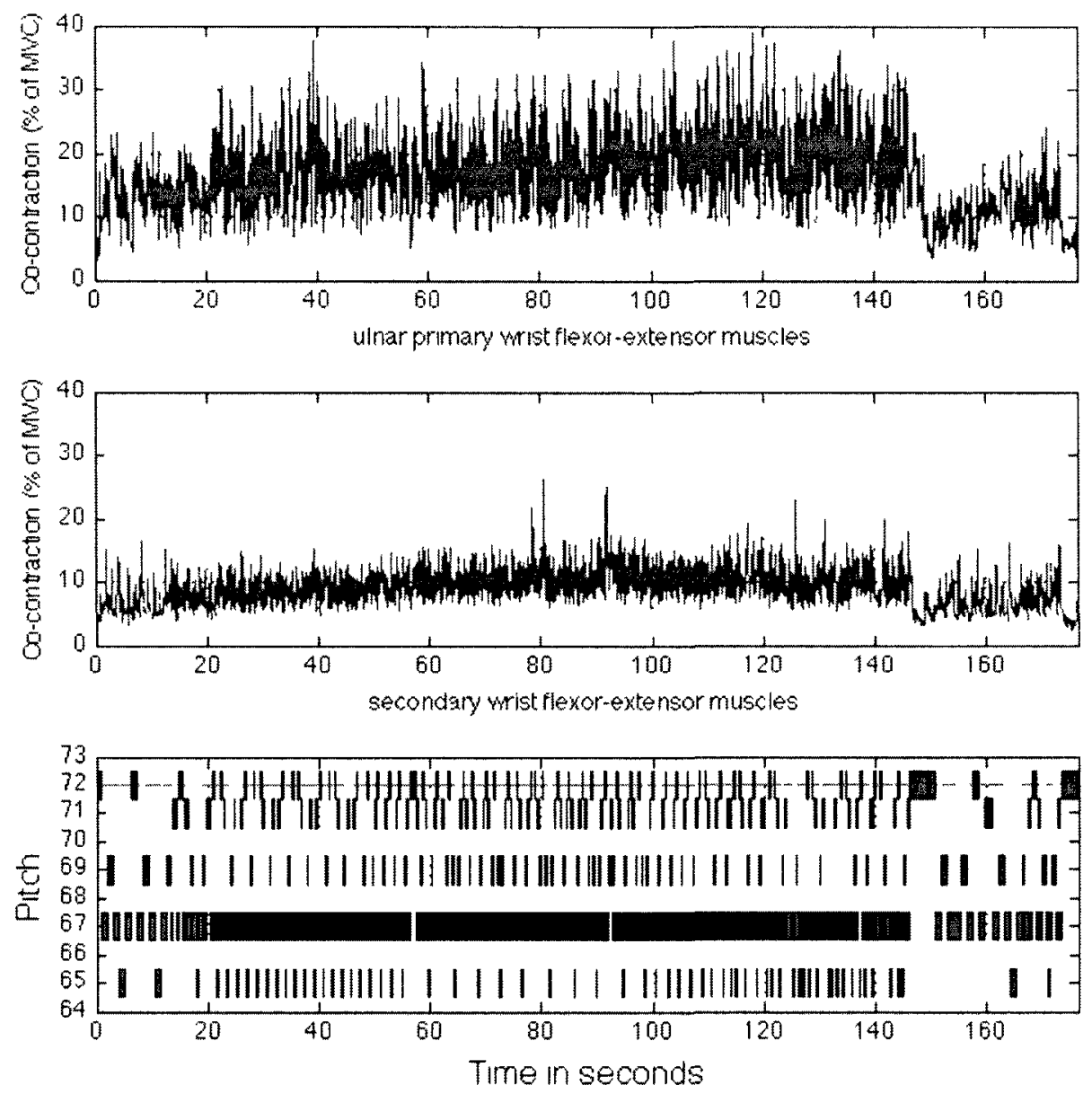

Figure P.6: Participant E004's EMG results: the upper plot shows levels of muscular co-contraction, expressed as a percentage of maximal voluntary contraction, while playing the Ann Southam piece for the second time for four muscles of the right forearm: the FCU, the ECU, the FDS and the EDC. The corresponding MIDI data (note on, note off) data is shown on the lower plot. 

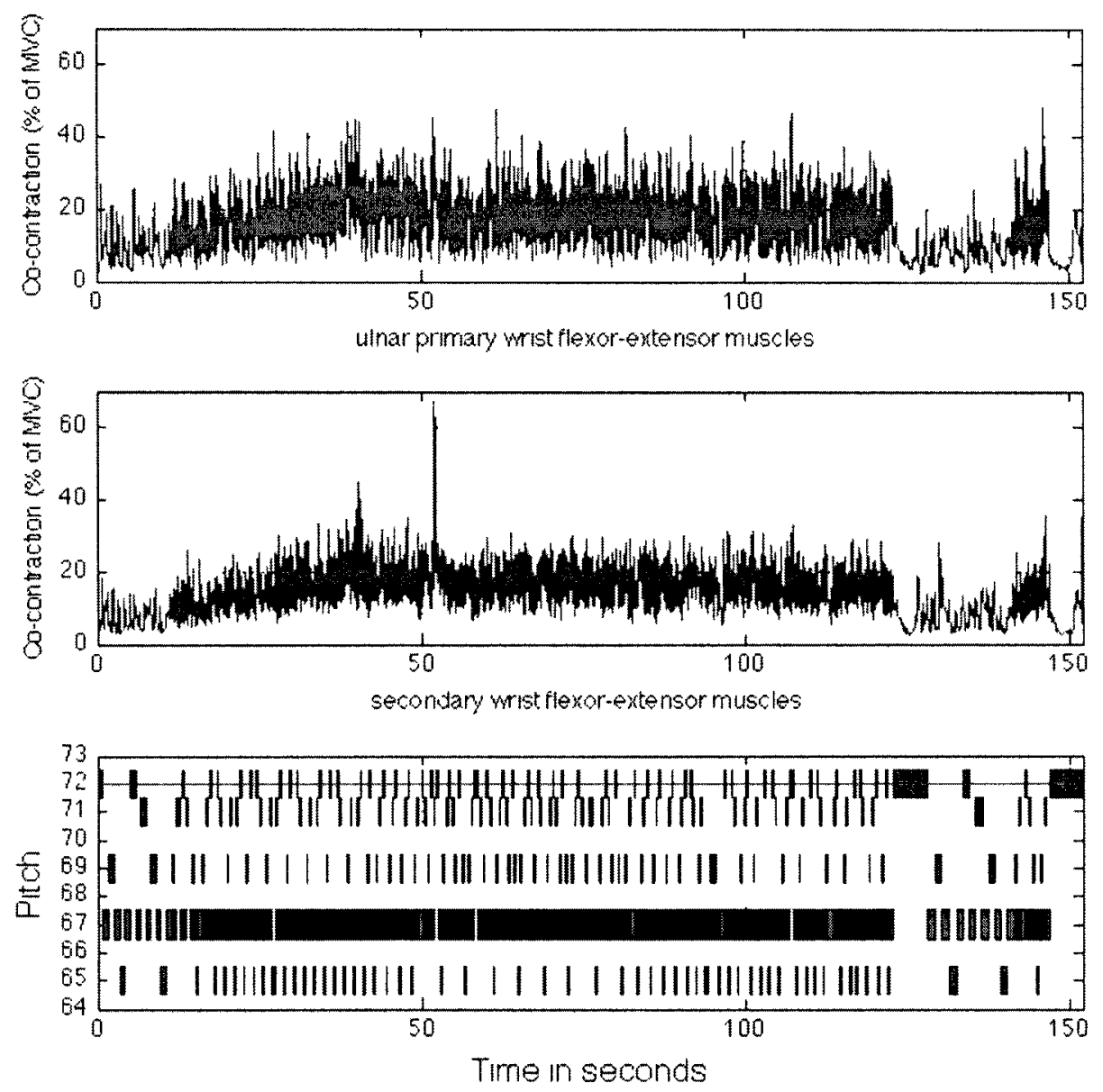

Figure P.7: Participant E005's EMG results: the upper plot shows levels of muscular co-contraction, expressed as a percentage of maximal voluntary contraction, while playing the Ann Southam piece for the first time for four muscles of the right forearm: the FCU, the ECU, the FDS and the EDC. The corresponding MIDI data (note on, note off) data is shown on the lower plot. 

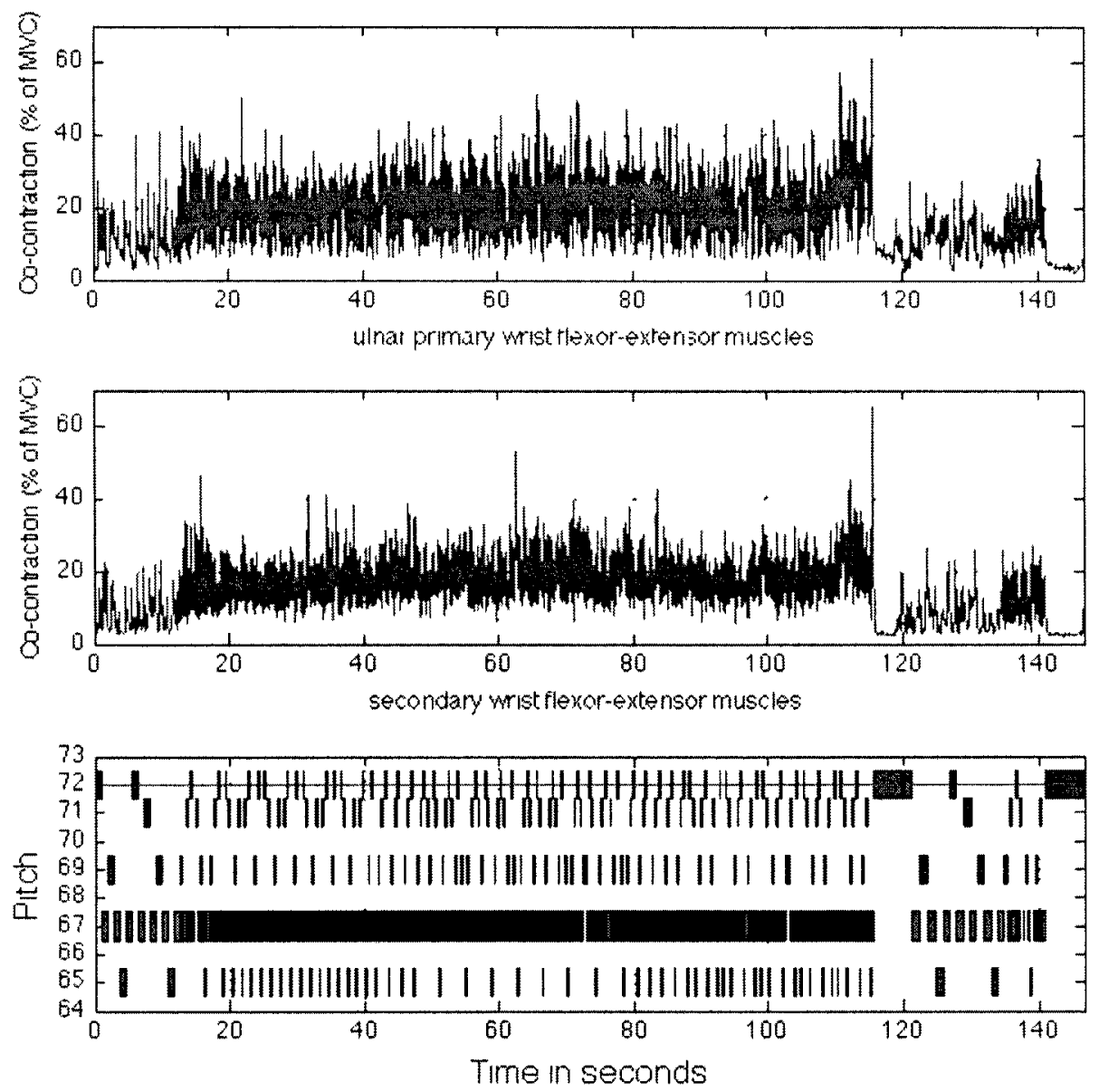

Figure P.8: Participant E005's EMG results: the upper plot shows levels of muscular co-contraction, expressed as a percentage of maximal voluntary contraction, while playing the Ann Southam piece for the second time for four muscles of the right forearm: the FCU, the ECU, the FDS and the EDC. The corresponding MIDI data (note on, note off) data is shown on the lower plot. 

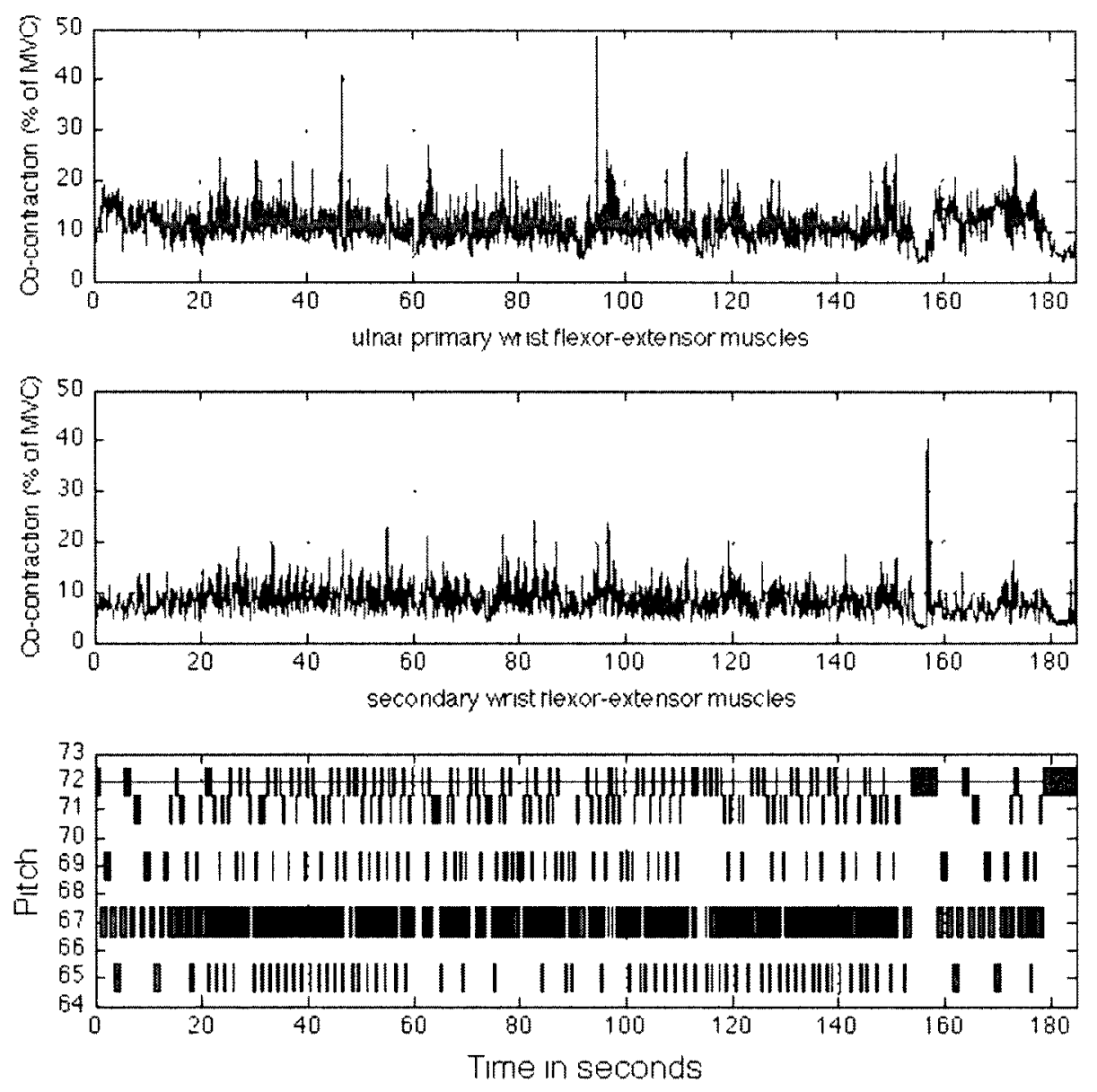

Figure P.9: Participant E006's EMG results: the upper plot shows levels of muscular co-contraction, expressed as a percentage of maximal voluntary contraction, while playing the Ann Southam piece for the first time for four muscles of the right forearm: the FCU, the ECU, the FDS and the EDC. The corresponding MIDI data (note on, note off) data is shown on the lower plot. 

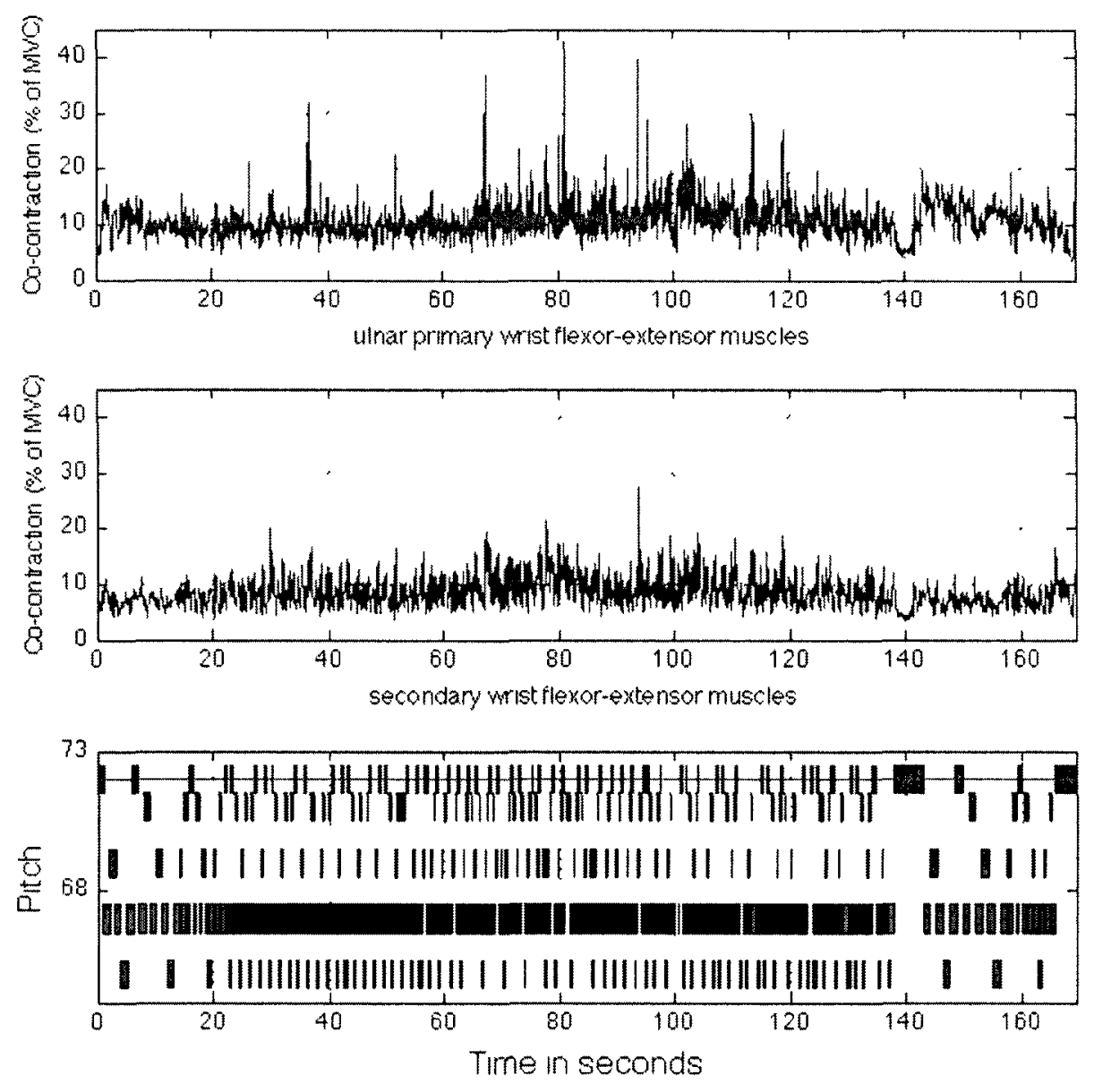

Figure P.10: Participant E006's EMG results: the upper plot shows levels of muscular co-contraction, expressed as a percentage of maximal voluntary contraction, while playing the Ann Southam piece for the second time for four muscles of the right forearm: the FCU, the ECU, the FDS and the EDC. The corresponding MIDI data (note on, note off) data is shown on the lower plot. 

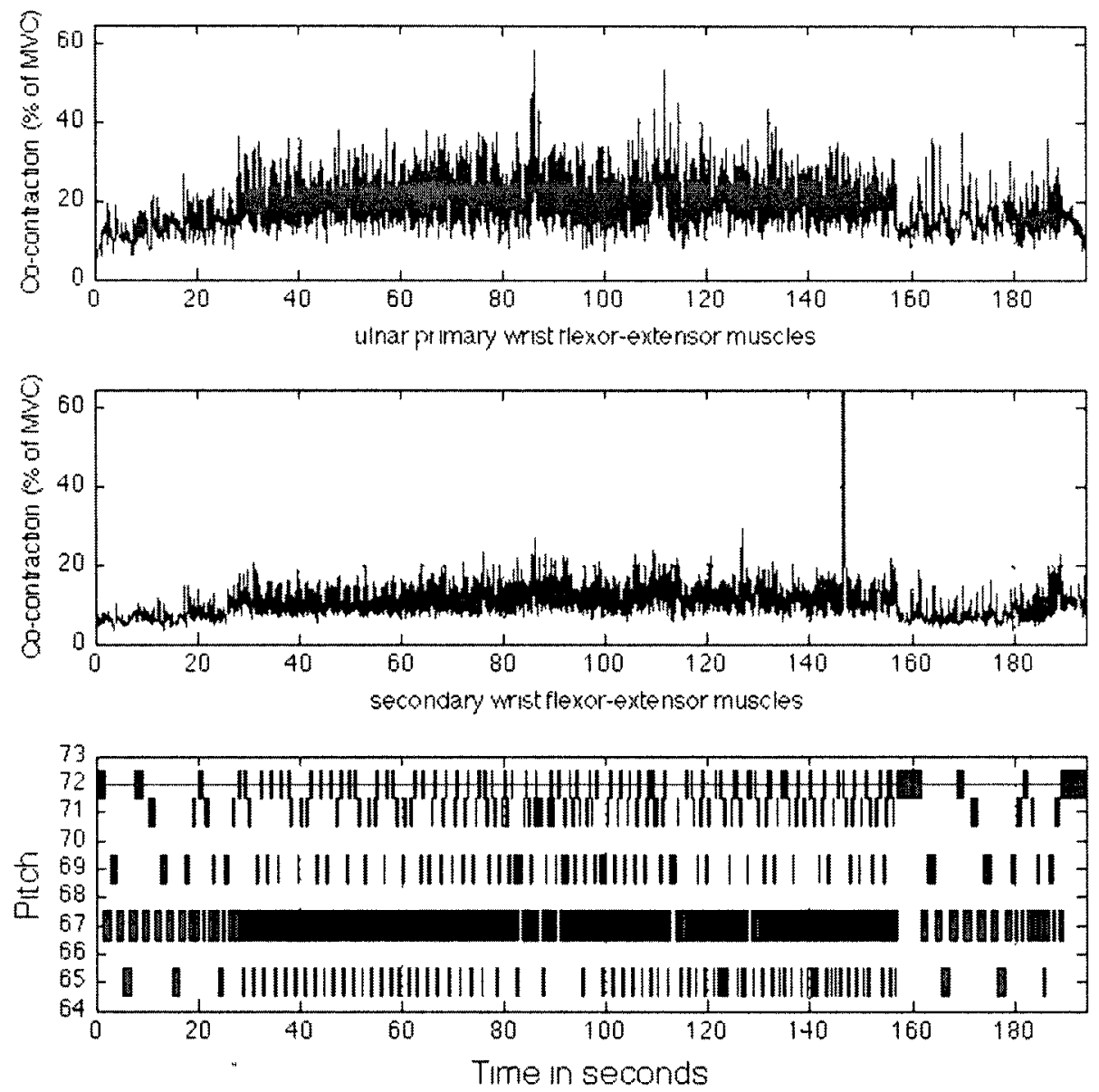

Figure P.11: Participant E007's EMG results: the upper plot shows levels of muscular co-contraction, expressed as a percentage of maximal voluntary contraction, while playing the Ann Southam piece for the first time for four muscles of the right forearm: the FCU, the ECU, the FDS and the EDC. The corresponding MIDI data (note on, note off) data is shown on the lower plot. 

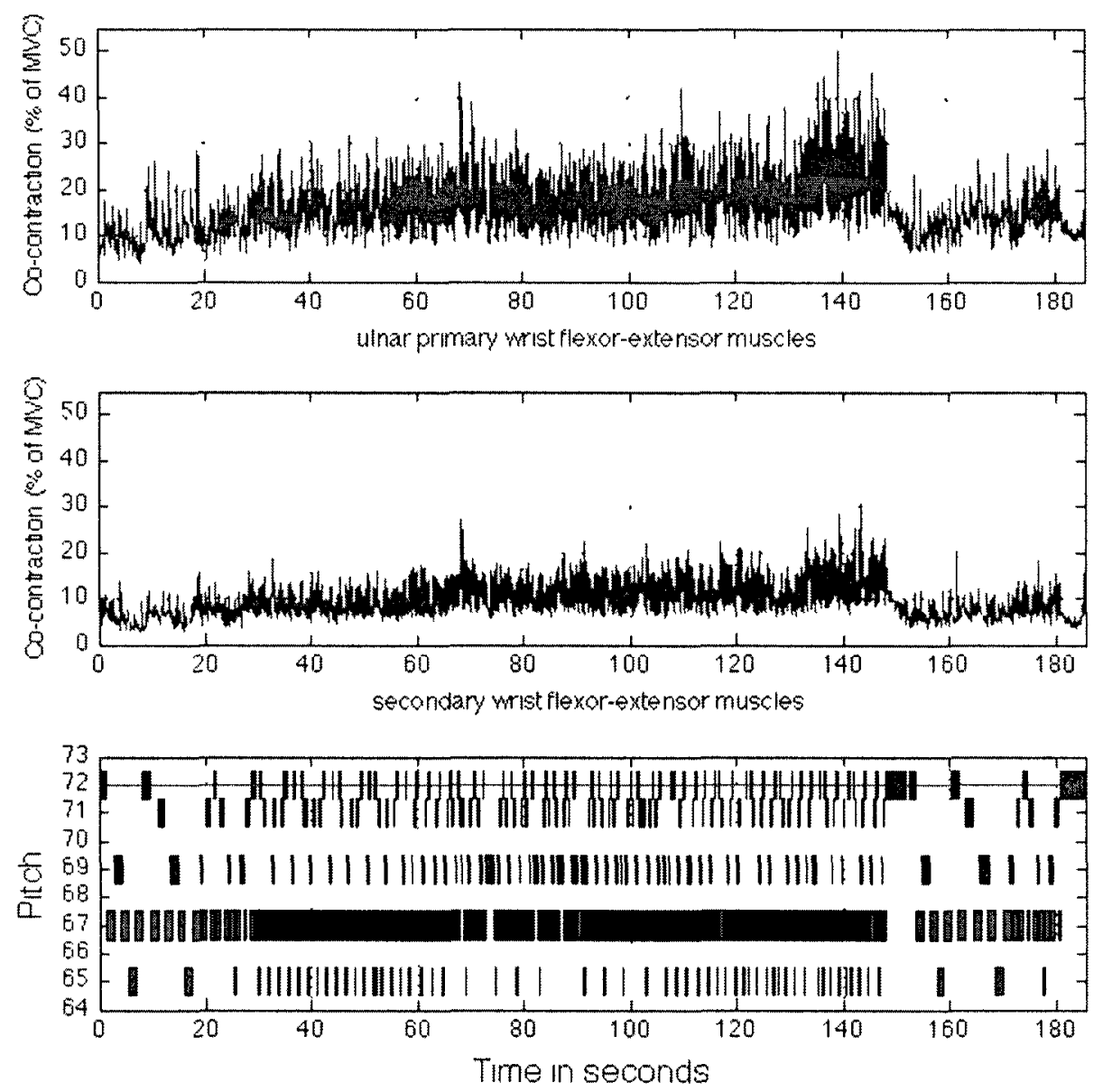

Figure P.12: Participant E007's EMG results: the upper plot shows levels of muscular co-contraction, expressed as a percentage of maximal voluntary contraction, while playing the Ann Southam piece for the second time for four muscles of the right forearm: the FCU, the ECU, the FDS and the EDC. The corresponding MIDI data (note on, note off) data is shown on the lower plot. 

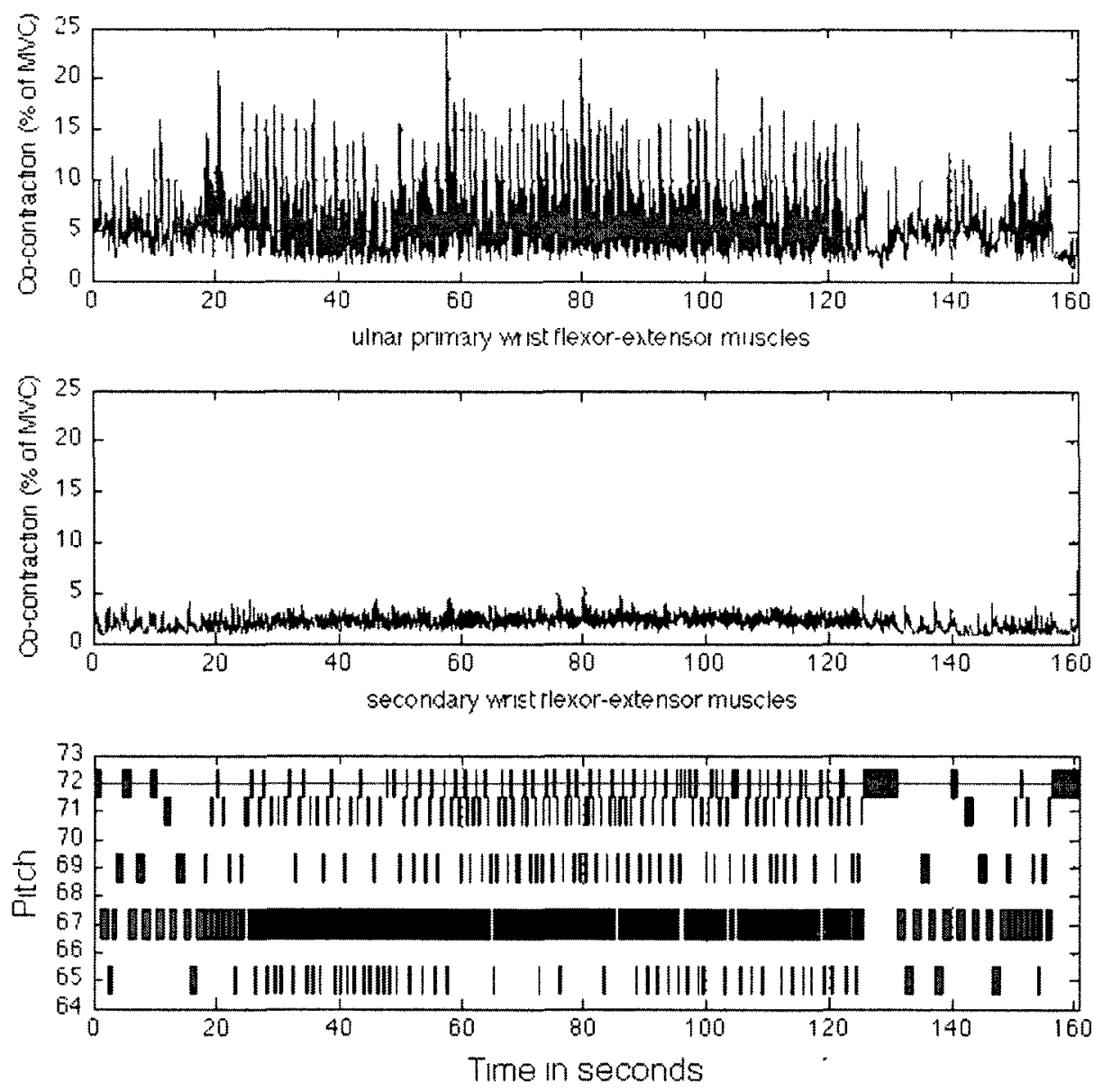

Figure P.13: Participant E008's EMG results the upper plot shows levels of muscular co-contraction, expressed as a percentage of maximal voluntary contraction, while playing the Ann Southam piece for the first time for four muscles of the right forearm the FCU, the ECU, the FDS and the EDC The corresponding MIDI data (note on, note off) data is shown on the lower plot. 

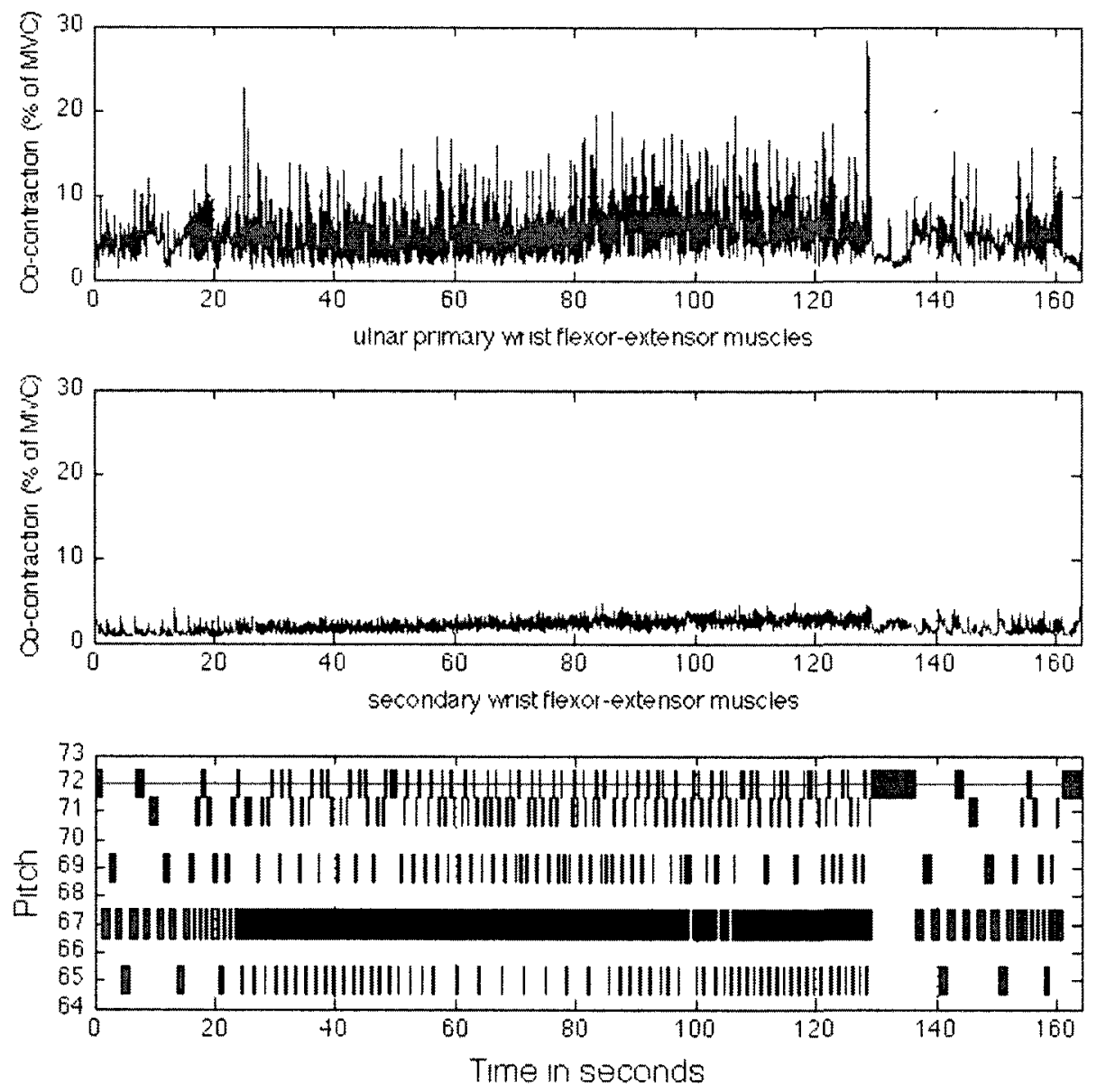

Figure P.14: Participant E008's EMG results the upper plot shows levels of muscular co-contraction, expressed as a percentage of maximal voluntary contraction, while playing the Ann Southam piece for the second time for four muscles of the right forearm: the FCU, the ECU, the FDS and the EDC. The corresponding MIDI data (note on, note off) data is shown on the lower plot. 

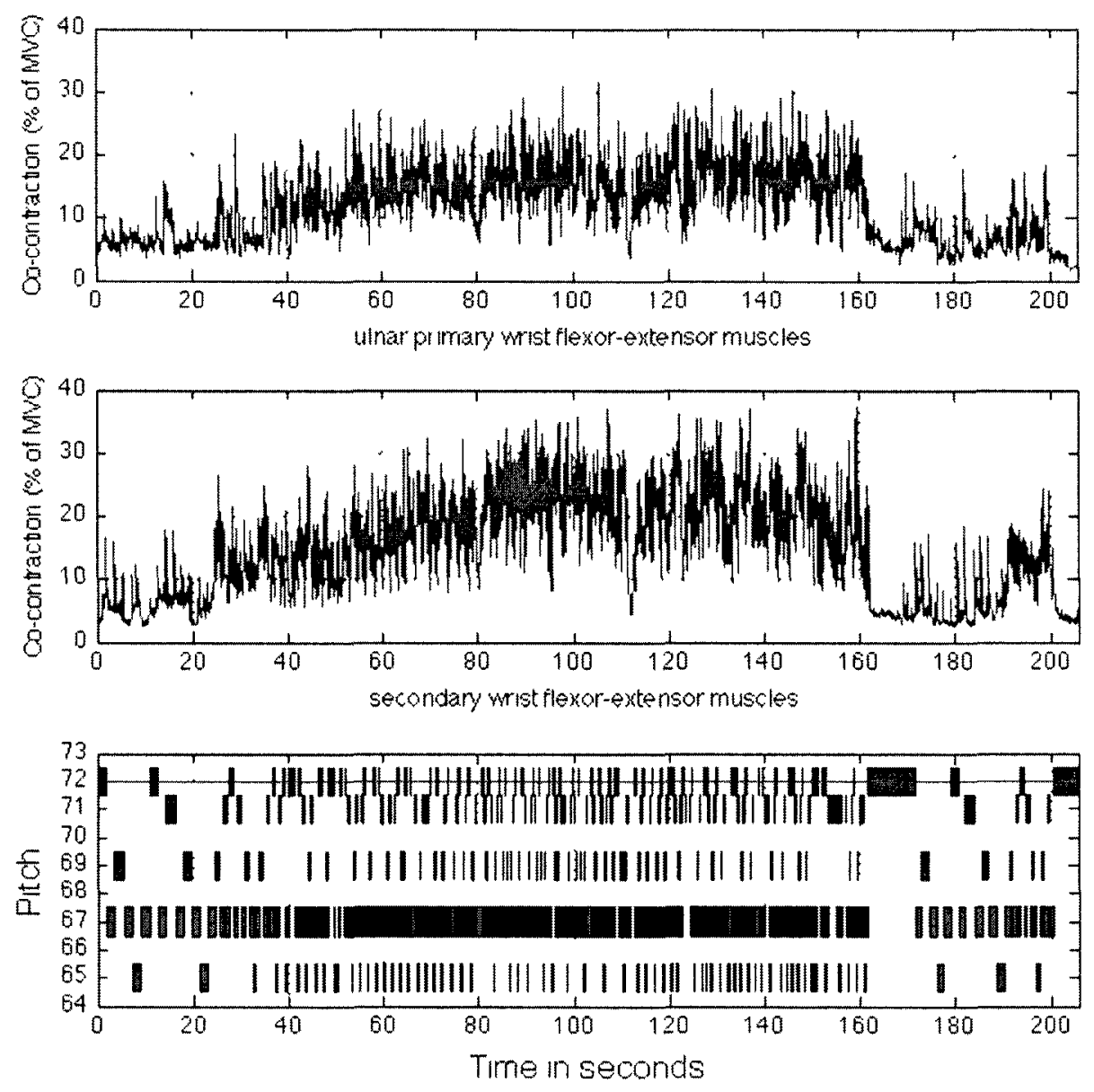

Figure P.15: Participant E009's EMG results: the upper plot shows levels of muscular co-contraction, expressed as a percentage of maximal voluntary contraction, while playing the Ann Southam piece for the first time for four muscles of the right forearm: the FCU, the ECU, the FDS and the EDC. The corresponding MIDI data (note on, note off) data is shown on the lower plot. 

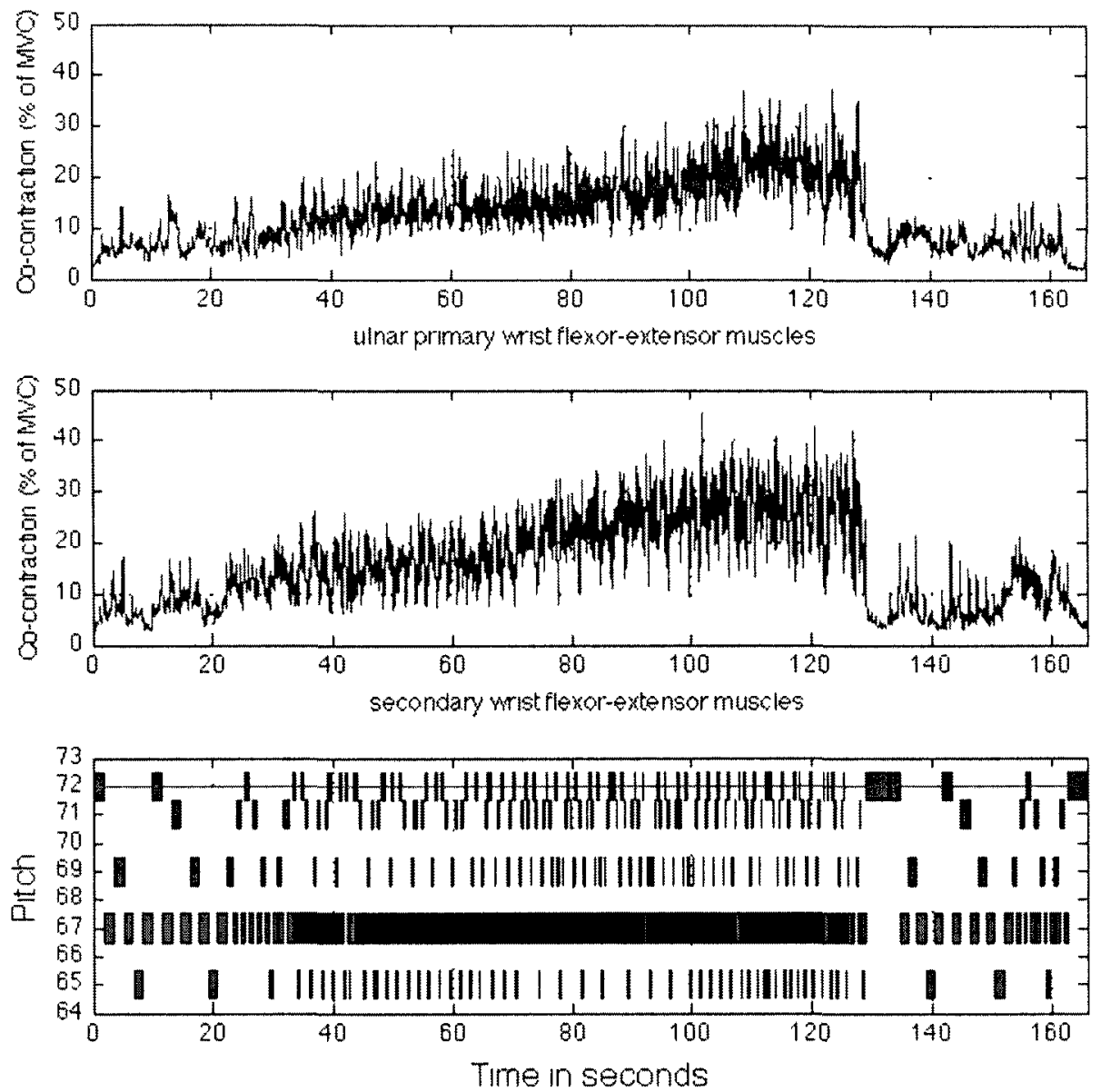

Figure P.16: Participant E009's EMG results: the upper plot shows levels of muscular co-contraction, expressed as a percentage of maximal voluntary contraction, while playing the Ann Southam piece for the second time for four muscles of the right forearm: the FCU, the ECU, the FDS and the EDC. The corresponding MIDI data (note on, note off) data is shown on the lower plot. 

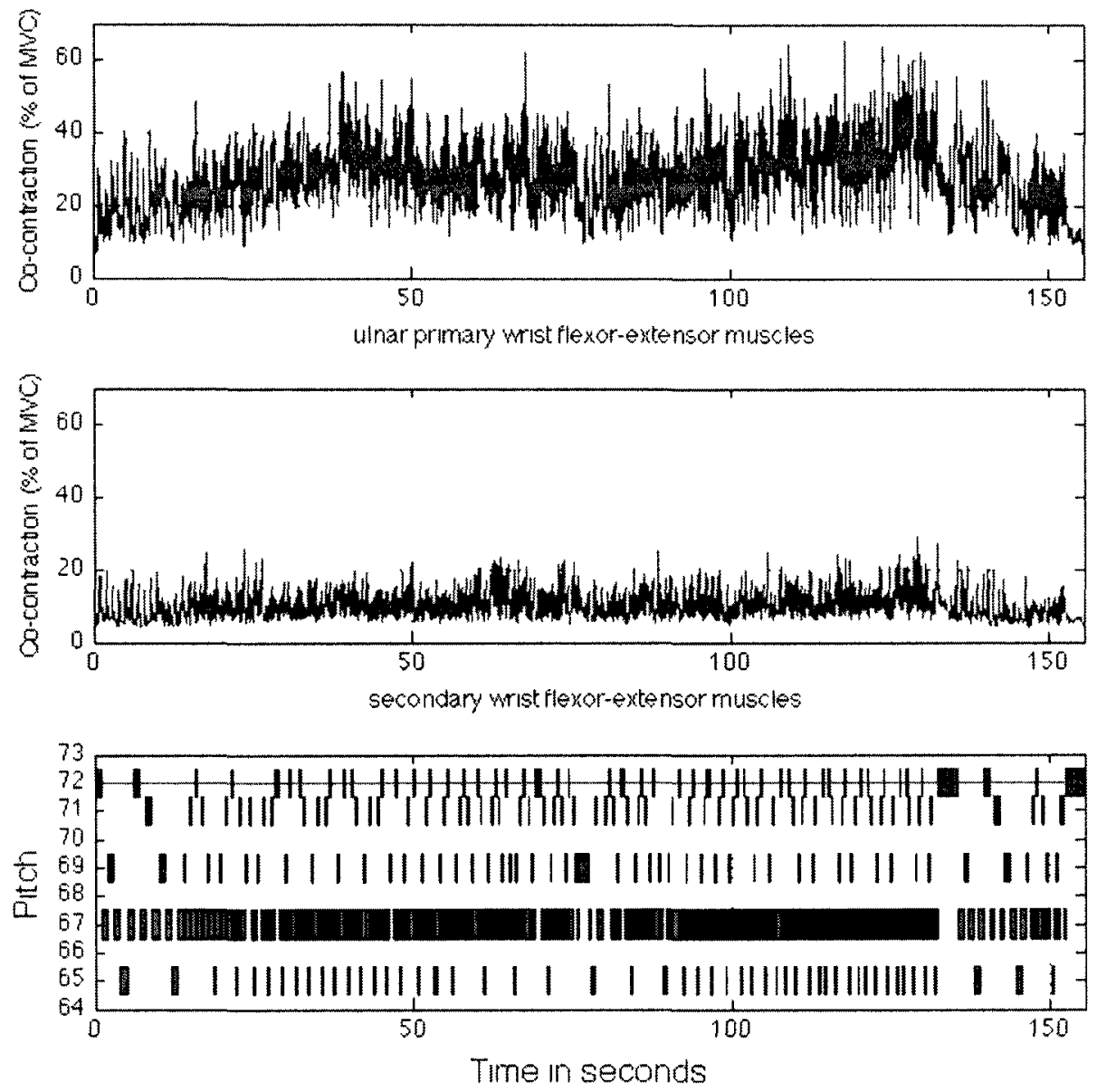

Figure P.17: Participant E010's EMG results: the upper plot shows levels of muscular co-contraction, expressed as a percentage of maximal voluntary contraction, while playing the Ann Southam piece for the first time for four muscles of the right forearm the FCU, the ECU, the FDS and the EDC. The corresponding MIDI data (note on, note off) data is shown on the lower plot 

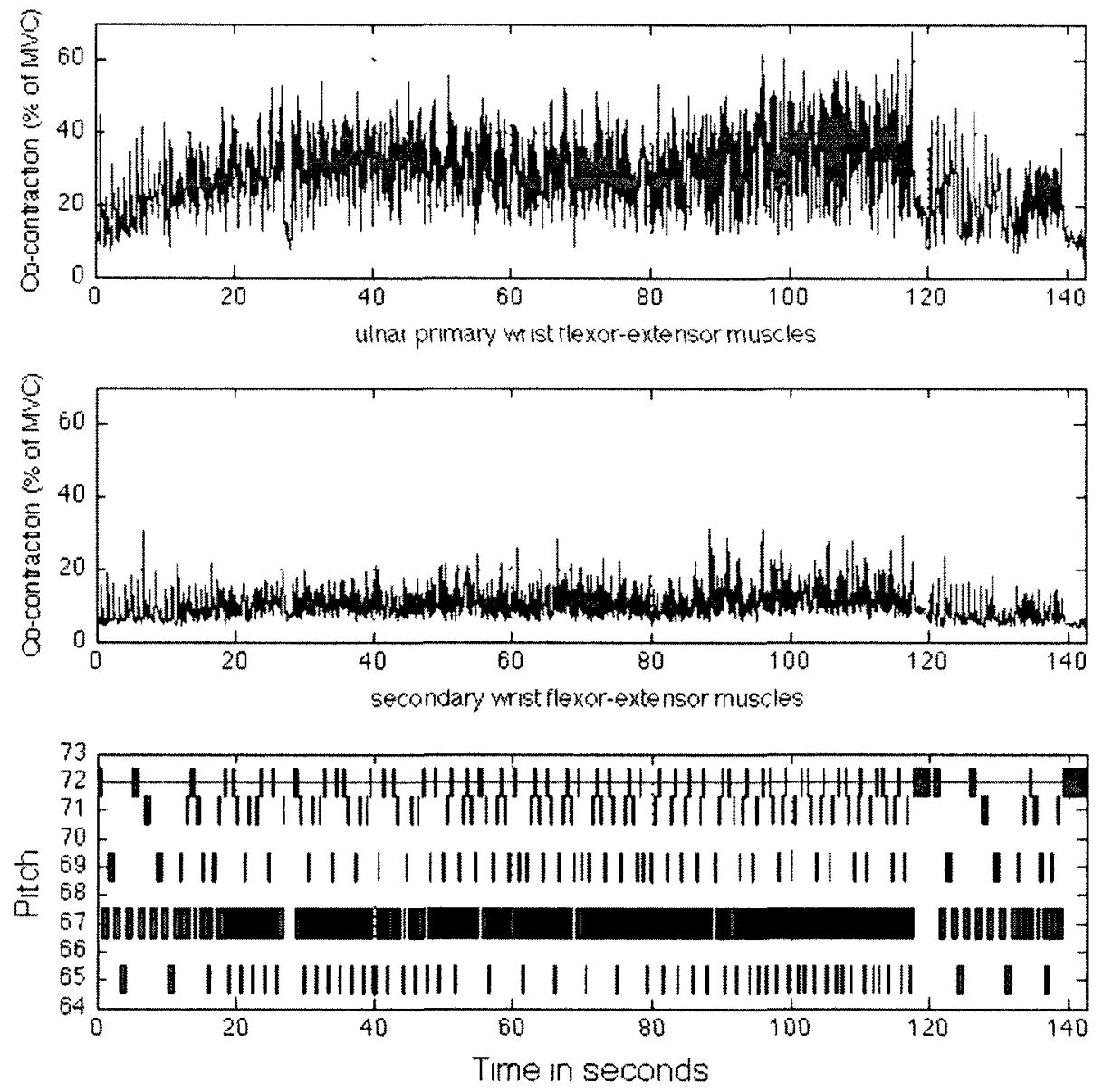

Figure P.18: Participant E010's EMG results: the upper plot shows levels of muscular co-contraction, expressed as a percentage of maximal voluntary contraction, while playing the Ann Southam piece for the second time for four muscles of the right forearm: the FCU, the ECU, the FDS and the EDC. The corresponding MIDI data (note on, note off) data is shown on the lower plot. 
Appendix Q

Participants' Mean Active Muscle

Stiffnesses and Mean Co-contraction

Levels for Sections A, A', B, B', W, W',

C96 and G96 of the Piece Composed by

Ann Southam 
Table Q.1: Mean active muscle stiffnesses of the FCU, ECU, FDS and EDC in sections A and A' of the Ann Southam piece. Results are expressed as a percentage of the MVC.

\begin{tabular}{|c|c|c|c|c|c|c|c|c|c|c|}
\hline & \multicolumn{8}{|c|}{ Mean Active Muscle Stiffness ( $\%$ of MVC) } \\
\hline & & & \multicolumn{4}{|c|}{$\mathbf{A}$} & \multicolumn{4}{|c|}{$A^{\prime}$} \\
\hline & & & FCU & $\mathrm{ECU}$ & FDS & $\mathrm{EDC}$ & $\mathrm{FCU}$ & ECU & FDS & EDC \\
\hline \multirow{18}{*}{ 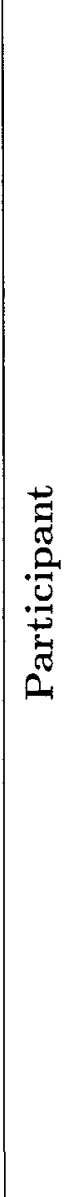 } & \multirow{9}{*}{ 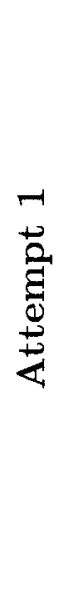 } & E002 & 13.46 & 28.76 & 6.30 & 10.21 & 8.16 & 27.2 & 4.19 & 7.73 \\
\hline & & E003 & 12.39 & 36.94 & 10.58 & 15.05 & 10.95 & 31.13 & 12.67 & 12.01 \\
\hline & & E004 & 12.54 & 14.99 & 4.02 & 11.68 & 12.18 & 17.03 & 3.49 & 16.98 \\
\hline & & E005 & 8.23 & 10.50 & 6.70 & 7.88 & 7.87 & 10.01 & 7.96 & 8.53 \\
\hline & & E006 & 12.13 & 14.49 & 9.02 & 6.38 & 13.76 & 12.66 & 8.81 & 5.36 \\
\hline & & E007 & 15.80 & 10.04 & 10.86 & 4.07 & 23.96 & 11.46 & 13.49 & 4.13 \\
\hline & & E008 & 3.81 & 8.49 & 0.90 & 4.06 & 4.15 & 7.28 & 0.78 & 3.84 \\
\hline & & E009 & 4.07 & 11.15 & 5.95 & 7.22 & 4.61 & 10.54 & 6.25 & 5.81 \\
\hline & & E010 & 23.83 & 15.71 & 10.21 & 6.85 & 40.12 & 20.67 & 9.62 & 8.2 \\
\hline & \multirow{9}{*}{ 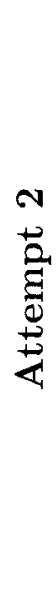 } & E002 & 6.80 & 27.36 & 4.37 & 7.39 & 7.49 & 26.58 & 4.5 & 7.89 \\
\hline & & E003 & 11.29 & 34.13 & 8.66 & 13.61 & 11.02 & 32.15 & 11.31 & 13.28 \\
\hline & & E004 & 12.39 & 13.66 & 3.56 & 12.47 & 9.22 & 12.26 & 3.47 & 13.49 \\
\hline & & E005 & 11.25 & 11.44 & 8.19 & 9.14 & 12.22 & 11.45 & 7.9 & 8.76 \\
\hline & & E006 & 8.44 & 13.04 & 8.58 & 6.05 & 12.95 & 12.79 & 8.51 & 5.92 \\
\hline & & $\mathrm{E} 007$ & 14.72 & 8.59 & 10.67 & 3.87 & 16.05 & 11.12 & 11.65 & 4.36 \\
\hline & & E008 & 3.86 & 6.23 & 0.66 & 2.94 & 4.09 & 7.76 & 0.93 & 3.98 \\
\hline & & E009 & 3.75 & 13.14 & 7.22 & 7.94 & 4.63 & 12.86 & 6.31 & 8.71 \\
\hline & & E010 & 26.52 & 15.54 & 9.51 & 7.78 & 32.24 & 16.48 & 9.1 & 7.07 \\
\hline
\end{tabular}


Table Q.2: Mean active muscle stiffnesses of the FCU, ECU, FDS and EDC in sections B and B' of the Ann Southam plece Results are expressed as a percentage of the MVC

\begin{tabular}{|c|c|c|c|c|c|c|c|c|c|c|}
\hline & \multicolumn{8}{|c|}{ Mean Active Muscle Stiffness (\% of MVC) } \\
\hline & & & \multicolumn{4}{|c|}{ B } & \multicolumn{4}{|c|}{$\mathrm{B}^{\prime}$} \\
\hline & & & FCU & $\mathrm{ECU}$ & FDS & EDC & FCU & $\mathrm{ECU}$ & FDS & EDC \\
\hline \multirow{18}{*}{ 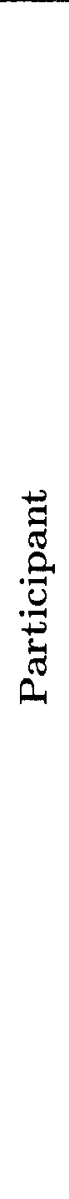 } & \multirow{9}{*}{ 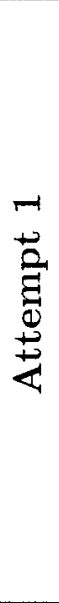 } & E002 & 1576 & 3314 & 860 & 1618 & 773 & 2880 & 462 & 985 \\
\hline & & E003 & 1499 & 4466 & 1619 & 2039 & 1285 & 3451 & 1429 & 1875 \\
\hline & & E004 & 1371 & 1537 & 463 & 1427 & 1461 & 1536 & 432 & 1432 \\
\hline & & E005 & 1223 & 1438 & 993 & 1210 & 1795 & 1701 & 1266 & 1648 \\
\hline & & E006 & 860 & 1363 & 979 & 707 & 1250 & 1353 & 984 & 655 \\
\hline & & E007 & 1815 & 1291 & 1294 & 550 & 1957 & 1401 & 1534 & 609 \\
\hline & & E008 & 490 & 998 & 083 & 456 & 449 & 835 & 079 & 347 \\
\hline & & E009 & 512 & 1409 & 1355 & 1241 & 493 & 1452 & 1292 & 1359 \\
\hline & & $\mathrm{E} 010$ & 3170 & 1858 & 1217 & 850 & 2817 & 1915 & 905 & 770 \\
\hline & \multirow{9}{*}{ 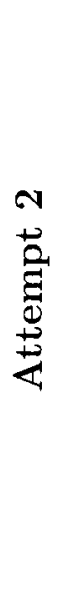 } & $\mathrm{E} 002$ & 898 & 2928 & 562 & 1317 & 1377 & 3096 & 638 & 1399 \\
\hline & & E003 & 1334 & 3883 & 1343 & 2010 & 1250 & 3458 & 1349 & 1799 \\
\hline & & E004 & 1163 & 1530 & 396 & 1458 & 1153 & 1342 & 423 & 1539 \\
\hline & & E005 & 1927 & 1902 & 1482 & 1829 & 1554 & 1557 & 1098 & 1393 \\
\hline & & E006 & 678 & 1214 & 895 & 648 & 909 & 1275 & 933 & 644 \\
\hline & & E007 & 1309 & 1123 & 1235 & 521 & 1848 & 1289 & 1399 & 553 \\
\hline & & E008 & 389 & 803 & 067 & 342 & 390 & 827 & 093 & 424 \\
\hline & & E009 & 526 & 1460 & 1270 & 1279 & 463 & 1480 & 1173 & 1305 \\
\hline & & $\mathrm{E} 010$ & 3513 & 1987 & 980 & 957 & 2831 & 1767 & 904 & 740 \\
\hline
\end{tabular}


Table Q.3: Mean active muscle stiffnesses of the FCU, ECU, FDS and EDC in sections $W$ and $W$ ' of the Ann Southam piece. Results are expressed as a percentage of the MVC.

\begin{tabular}{|c|c|c|c|c|c|c|c|c|c|c|}
\hline & \multicolumn{8}{|c|}{ Mean Active Muscle Stiffness (\% of MVC) } \\
\hline & & & \multicolumn{4}{|c|}{$\mathbf{W}$} & \multicolumn{4}{|c|}{$\mathbf{W}^{\prime}$} \\
\hline & & & FCU & ECU & FDS & EDC & FCU & ECU & FDS & EDC \\
\hline \multirow{18}{*}{ 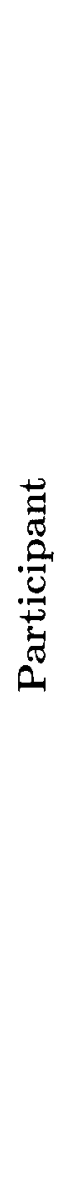 } & \multirow{9}{*}{ 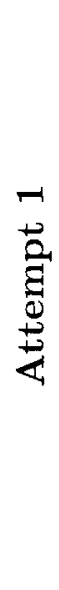 } & E002 & 23.78 & 31.06 & 11.12 & 15.14 & 10.55 & 25.61 & 3.26 & 4.78 \\
\hline & & E003 & 12.06 & 22.78 & 9.59 & 9.88 & 8.87 & 24.57 & 5.49 & 3.33 \\
\hline & & E004 & 12.01 & 12.68 & 2.81 & 11.61 & 17.89 & 14.79 & 2.61 & 9.34 \\
\hline & & E005 & 10.22 & 7.00 & 7.07 & 7.02 & 7.72 & 8.01 & 6.31 & 7.75 \\
\hline & & E006 & 11.88 & 4.62 & 13.85 & 3.43 & 11.77 & 4.53 & 11.81 & 4.05 \\
\hline & & $\mathrm{E} 007$ & 19.64 & 8.21 & 10.59 & 4.42 & 22.02 & 9.90 & 14.25 & 8.33 \\
\hline & & E008 & 4.51 & 4.99 & 0.90 & 5.61 & 5.00 & 1.83 & 0.48 & 5.11 \\
\hline & & E009 & 5.18 & 8.21 & 3.28 & 6.90 & 2.10 & 5.22 & 2.94 & 6.76 \\
\hline & & E010 & 44.20 & 16.73 & 13.25 & 9.72 & 18.77 & 8.57 & 7.24 & 5.22 \\
\hline & \multirow{9}{*}{ 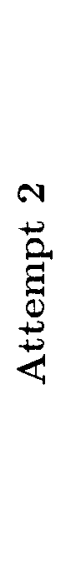 } & E002 & 21.67 & 29.70 & 9.77 & 15.13 & 15.11 & 26.65 & 6.90 & 8.32 \\
\hline & & E003 & 11.91 & 25.27 & 8.86 & 11.02 & 8.50 & 23.59 & 5.17 & 3.11 \\
\hline & & E004 & 16.33 & 15.62 & 2.76 & 12.30 & 4.53 & 8.92 & 2.49 & 6.91 \\
\hline & & E005 & 17.04 & 6.16 & 5.70 & 3.74 & 4.09 & 4.26 & 4.01 & 2.26 \\
\hline & & E006 & 10.30 & 4.72 & 7.00 & 4.26 & 10.40 & 6.24 & 13.57 & 5.78 \\
\hline & & E007 & 23.78 & 11.26 & 14.15 & 6.39 & 20.19 & 7.05 & 7.38 & 4.63 \\
\hline & & E008 & 3.64 & 4.11 & 1.03 & 4.41 & 4.77 & 1.76 & 0.74 & 4.54 \\
\hline & & E009 & 4.31 & 11.22 & 3.26 & 7.86 & 1.24 & 4.62 & 5.04 & 6.19 \\
\hline & & E010 & 32.82 & 13.16 & 11.97 & 7.52 & 18.50 & 7.94 & 5.45 & 5.47 \\
\hline
\end{tabular}


Table Q.4: Mean active muscle stiffnesses of the FCU, ECU, FDS and EDC in sections C96 and G96 of the Ann Southam piece. Results are expressed as a percentage of the MVC.

\begin{tabular}{|c|c|c|c|c|c|c|c|c|c|c|}
\hline & \multicolumn{8}{|c|}{ Mean Active Muscle Stiffness (\% of MVC) } \\
\hline & & & \multicolumn{4}{|c|}{ C96 } & \multicolumn{4}{|c|}{ G96 } \\
\hline & & & FCU & $\mathrm{ECU}$ & FDS & EDC & FCU & $\mathrm{ECU}$ & FDS & EDC \\
\hline \multirow{18}{*}{ 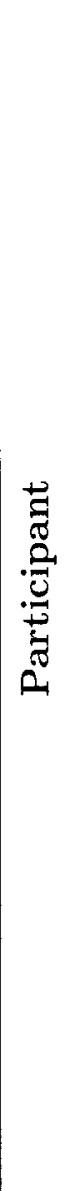 } & \multirow{9}{*}{ 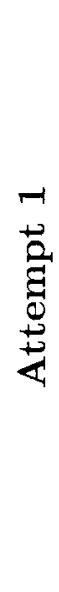 } & E002 & 18.13 & 34.96 & 9.39 & 17.18 & 29.03 & 44.35 & 19.90 & 28.50 \\
\hline & & E003 & 17.13 & 49.44 & 17.19 & 20.86 & 24.10 & 57.16 & 25.57 & 30.12 \\
\hline & & E004 & 16.60 & 20.66 & 5.33 & 16.34 & 18.91 & 20.97 & 6.09 & 17.26 \\
\hline & & E005 & 22.04 & 18.85 & 17.62 & 16.83 & 20.01 & 18.22 & 15.90 & 17.30 \\
\hline & & E006 & 10.68 & 14.65 & 11.86 & 7.95 & 9.61 & 13.48 & 10.89 & 6.55 \\
\hline & & E007 & 25.13 & 17.17 & 16.50 & 7.13 & 22.72 & 19.63 & 38.73 & 7.09 \\
\hline & & E008 & 3.89 & 9.83 & 0.99 & 5.31 & 3.72 & 10.51 & 1.01 & 5.99 \\
\hline & & E009 & 8.14 & 23.64 & 13.98 & 17.55 & 9.49 & 30.98 & 17.66 & 23.07 \\
\hline & & E010 & 38.69 & 24.65 & 10.80 & 10.55 & 44.51 & 31.48 & 12.77 & 12.54 \\
\hline & \multirow{9}{*}{ 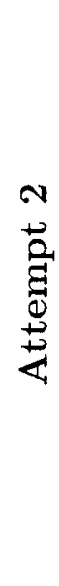 } & E002 & 18.13 & 34.96 & 9.39 & 17.18 & 27.55 & 42.53 & 16.74 & 25.54 \\
\hline & & E003 & 18.74 & 53.84 & 19.32 & 22.99 & 26.20 & 57.99 & 30.15 & 32.33 \\
\hline & & E004 & 16.01 & 19.71 & 4.71 & 15.75 & 20.18 & 22.43 & 6.10 & 17.07 \\
\hline & & E005 & 21.04 & 19.65 & 16.69 & 19.04 & 26.44 & 22.17 & 19.16 & 20.89 \\
\hline & & E006 & 7.86 & 13.28 & 10.60 & 7.22 & 9.64 & 13.48 & 11.03 & 7.28 \\
\hline & & E007 & 17.01 & 15.56 & 12.99 & 6.38 & 26.62 & 20.81 & 22.93 & 8.26 \\
\hline & & E008 & 3.71 & 9.24 & 0.73 & 4.88 & 4.91 & 12.27 & 1.07 & 7.65 \\
\hline & & E009 & 7.81 & 23.42 & 13.88 & 17.63 & 12.64 & 40.10 & 24.55 & 29.87 \\
\hline & & E010 & 39.63 & 25.37 & 11.10 & 10.80 & 46.71 & 30.55 & 13.81 & 13.95 \\
\hline
\end{tabular}


Table Q.5: Mean co-contraction levels (CC1 and CC2) in sections $\mathrm{A}$ and $\mathrm{A}^{\prime}$ of the Ann Southam piece. Results are expressed as a percentage of the MVC.

\begin{tabular}{|c|c|c|c|c|c|c|}
\hline & \multicolumn{4}{|c|}{ Mean Co-contraction ( $\%$ of MVC) } \\
\hline & & & \multicolumn{2}{|c|}{ A } & \multicolumn{2}{|c|}{$\mathrm{A}^{\prime}$} \\
\hline & & & $\mathrm{CC} 1$ & $\mathrm{CC} 2$ & $\mathrm{CC} 1$ & $\mathrm{CC2}$ \\
\hline \multirow{18}{*}{ 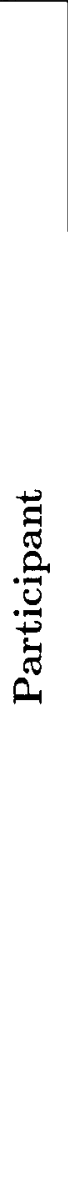 } & \multirow{9}{*}{ 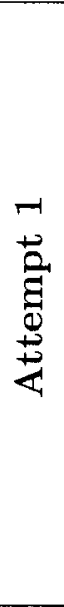 } & E002 & 19.19 & 7.87 & 14.55 & 5.59 \\
\hline & & E003 & 21.07 & 12.17 & 18.13 & 11.30 \\
\hline & & E004 & 13.38 & 6.76 & 13.89 & 7.58 \\
\hline & & E005 & 9.00 & 7.02 & 8.43 & 7.92 \\
\hline & & E006 & 13.04 & 7.51 & 12.93 & 6.80 \\
\hline & & E007 & 12.36 & 6.53 & 16.2 & 7.30 \\
\hline & & E008 & 5.50 & 1.84 & 5.13 & 1.64 \\
\hline & & E009 & 6.57 & 6.27 & 6.61 & 5.76 \\
\hline & & E010 & 18.53 & 7.93 & 27.37 & 8.53 \\
\hline & \multirow{9}{*}{ 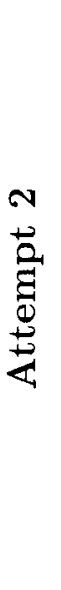 } & E002 & 13.48 & 5.53 & 13.79 & 5.78 \\
\hline & & E003 & 19.25 & 10.30 & 18.46 & 11.38 \\
\hline & & E004 & 12.68 & 6.55 & 10.19 & 6.62 \\
\hline & & E005 & 10.97 & 8.31 & 11.19 & 7.83 \\
\hline & & E006 & 10.19 & 7.14 & 12.60 & 7.03 \\
\hline & & E007 & 10.88 & 6.30 & 13.05 & 6.92 \\
\hline & & E008 & 4.75 & 1.32 & 5.29 & 1.82 \\
\hline & & E009 & 6.72 & 7.33 & 7.40 & 7.03 \\
\hline & & E010 & 19.75 & 8.21 & 21.60 & 7.70 \\
\hline
\end{tabular}


Table Q.6: Mean co-contraction levels (CC1 and CC2) in sections B and B' of the Ann Southam piece. Results are expressed as a percentage of the MVC.

\begin{tabular}{|c|c|c|c|c|c|c|}
\hline & \multicolumn{4}{|c|}{ Mean Co-contraction ( $\%$ of MVC) } \\
\hline & & & \multicolumn{2}{|c|}{$\mathrm{B}$} & \multicolumn{2}{|c|}{$\mathrm{B}^{\prime}$} \\
\hline & & & $\mathrm{CC} 1$ & $\mathrm{CC} 2$ & $\mathrm{CC} 1$ & $\mathrm{CC} 2$ \\
\hline \multirow{18}{*}{ 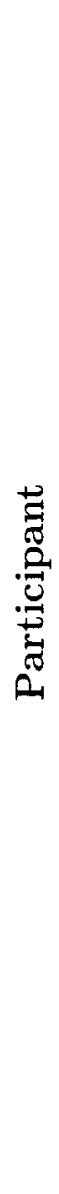 } & \multirow{9}{*}{ 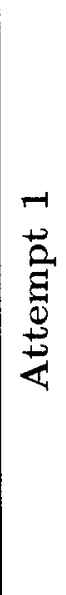 } & E002 & 22.52 & 11.64 & 14.70 & 6.66 \\
\hline & & E003 & 25.44 & 17.34 & 20.74 & 15.67 \\
\hline & & E004 & 14.01 & 8.02 & 14.34 & 7.79 \\
\hline & & E005 & 12.62 & 10.80 & 16.98 & 14.27 \\
\hline & & E006 & 10.47 & 8.25 & 12.61 & 7.96 \\
\hline & & E007 & 15.15 & 8.35 & 16.12 & 9.51 \\
\hline & & E008 & 6.61 & 1.87 & 5.70 & 1.59 \\
\hline & & E009 & 8.07 & 12.67 & 7.92 & 12.99 \\
\hline & & E010 & 23.49 & 9.77 & 22.05 & 8.12 \\
\hline & \multirow{9}{*}{ 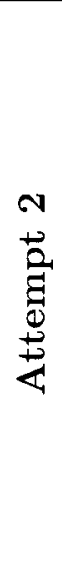 } & E002 & 16.06 & 8.45 & 20.00 & 9.29 \\
\hline & & E003 & 22.32 & 15.37 & 20.38 & 14.79 \\
\hline & & E004 & 12.96 & 7.53 & 12.10 & 7.96 \\
\hline & & E005 & 18.56 & 16.24 & 14.76 & 11.98 \\
\hline & & E006 & 8.78 & 7.54 & 10.40 & 7.65 \\
\hline & & $\mathrm{E} 007$ & 11.91 & 7.89 & 15.17 & 8.65 \\
\hline & & E008 & 5.21 & 1.45 & 5.23 & 1.89 \\
\hline & & E009 & 8.36 & 12.48 & 7.73 & 12.05 \\
\hline & & E010 & 25.90 & 9.41 & 21.24 & 7.98 \\
\hline
\end{tabular}


Table Q.7: Mean co-contraction levels (CC1 and CC2) in sections W and W' of the Ann Southam piece. Results are expressed as a percentage of the MVC.

\begin{tabular}{|c|c|c|c|c|c|c|}
\hline & \multicolumn{4}{|c|}{ Mean Co-contraction (\% of MVC) } \\
\hline & & & \multicolumn{2}{|c|}{$\mathbf{W}$} & \multicolumn{2}{|c|}{$\mathrm{W}^{\prime}$} \\
\hline & & & $\mathrm{CC} 1$ & $\mathrm{CC} 2$ & $\mathrm{CC} 1$ & $\mathrm{CC} 2$ \\
\hline \multirow{18}{*}{ 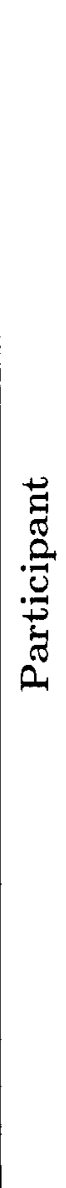 } & \multirow{9}{*}{ 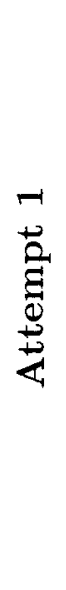 } & E002 & 26.90 & 12.86 & 16.33 & 3.93 \\
\hline & & E003 & 16.38 & 9.07 & 14.68 & 4.07 \\
\hline & & E004 & 12.03 & 5.60 & 16.05 & 4.84 \\
\hline & & E005 & 7.97 & 6.26 & 7.43 & 6.59 \\
\hline & & E006 & 6.55 & 6.20 & 6.95 & 6.01 \\
\hline & & E007 & 12.33 & 6.80 & 14.60 & 10.85 \\
\hline & & E008 & 3.62 & 2.18 & 2.74 & 1.53 \\
\hline & & E009 & 6.18 & 4.69 & 3.25 & 4.38 \\
\hline & & E010 & 25.67 & 11.24 & 12.23 & 6.06 \\
\hline & \multirow{9}{*}{ 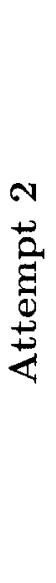 } & E002 & 25.08 & 12.09 & 19.87 & 7.54 \\
\hline & & E003 & 16.98 & 9.48 & 14.06 & 3.72 \\
\hline & & E004 & 15.53 & 5.75 & 6.31 & 4.10 \\
\hline & & E005 & 9.27 & 4.44 & 4.05 & 2.92 \\
\hline & & E006 & 6.42 & 5.43 & 7.81 & 8.80 \\
\hline & & E007 & 16.21 & 9.44 & 11.53 & 5.82 \\
\hline & & E008 & 2.78 & 2.09 & 2.63 & 1.78 \\
\hline & & E009 & 6.76 & 5.00 & 2.35 & 5.45 \\
\hline & & E010 & 19.73 & 9.35 & 11.77 & 5.41 \\
\hline
\end{tabular}


Table Q.8: Mean co-contraction levels (CC1 and $\mathrm{CC} 2)$ in sections $\mathrm{C} 96$ and $\mathrm{G} 96$ of the Ann Southam piece. Results are expressed as a percentage of the MVC.

\begin{tabular}{|c|c|c|c|c|c|c|}
\hline & \multicolumn{4}{|c|}{ Mean Co-contraction ( $\%$ of MVC) } \\
\hline & & & \multicolumn{2}{|c|}{$\mathrm{C} 96$} & \multicolumn{2}{|c|}{ G96 } \\
\hline & & & $\mathrm{CC} 1$ & $\mathrm{CC} 2$ & $\mathrm{CC} 1$ & $\mathrm{CC} 2$ \\
\hline \multirow{18}{*}{ 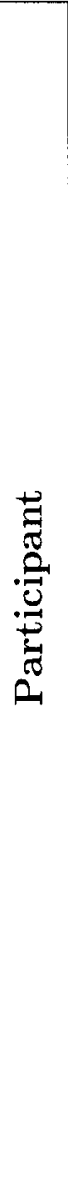 } & \multirow{9}{*}{$\begin{array}{l}\vec{~} \\
\vec{a}\end{array}$} & E002 & 24.77 & 12.50 & 35.33 & 23.45 \\
\hline & & E003 & 28.59 & 18.34 & 36.32 & 26.31 \\
\hline & & E004 & 17.84 & 9.19 & 19.21 & 10.13 \\
\hline & & E005 & 19.85 & 16.95 & 18.48 & 16.34 \\
\hline & & E006 & 12.08 & 9.60 & 10.98 & 8.38 \\
\hline & & E007 & 20.40 & 10.72 & 20.63 & 13.32 \\
\hline & & E008 & 5.49 & 2.23 & 5.60 & 2.40 \\
\hline & & E009 & 13.35 & 15.03 & 16.35 & 19.52 \\
\hline & & E010 & 29.98 & 10.22 & 36.03 & 12.11 \\
\hline & \multirow{9}{*}{ 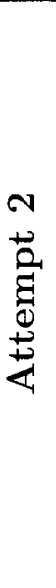 } & E002 & 24.77 & 12.50 & 33.60 & 20.36 \\
\hline & & E003 & 31.07 & 20.26 & 37.93 & 29.45 \\
\hline & & E004 & 17.05 & 8.50 & 20.56 & 10.05 \\
\hline & & E005 & 19.63 & 17.54 & 23.34 & 19.69 \\
\hline & & E006 & 9.81 & 8.66 & 10.98 & 8.85 \\
\hline & & E007 & 15.92 & 8.98 & 22.90 & 13.53 \\
\hline & & E008 & 5.27 & 1.82 & 6.99 & 2.78 \\
\hline & & E009 & 13.01 & 15.07 & 21.83 & 26.23 \\
\hline & & E010 & 30.73 & 10.51 & 36.28 & 13.21 \\
\hline
\end{tabular}

